\title{
Labraunda 2017
}

Olivier Henry, Erika Andersson, Jesper Blid, Christophe Bost, Ömür Dünya Çakmaklı, Naomi Carless-Unwin, Görkem Çimen, Ayşe Eyigör, Agneta Freccero, Axel Frejman, Cristina Georgescu, Élisabeth Goussard, AnneMarie Guimier-Sorbets, Mélanie Hauchart, Ragnar Hedlund, Nicolas Lamare, Vasilica Lungu, Frédérique Marchand-Beaulieu, Anna Sitz, Ivana Stojanovic et Baptiste Vergnaud

\section{(2) OpenEdition}

\section{Journals}

Édition électronique

URL : https://journals.openedition.org/anatoliaantiqua/657

DOI : 10.4000/anatoliaantiqua.657

Éditeur

IFEA

Édition imprimée

Date de publication : 1 décembre 2018

Pagination : 209-301

ISBN : 9782362450747

ISSN : 1018-1946

Référence électronique

Olivier Henry, Erika Andersson, Jesper Blid, Christophe Bost, Ömür Dünya Çakmaklı, Naomi CarlessUnwin, Görkem Çimen, Ayşe Eyigör, Agneta Freccero, Axel Frejman, Cristina Georgescu, Élisabeth Goussard, Anne-Marie Guimier-Sorbets, Mélanie Hauchart, Ragnar Hedlund, Nicolas Lamare, Vasilica Lungu, Frédérique Marchand-Beaulieu, Anna Sitz, Ivana Stojanovic et Baptiste Vergnaud, « Labraunda 2017 », Anatolia Antiqua [En ligne], XXVI | 2018, mis en ligne le 16 décembre 2019, consulté le 03 septembre 2021. URL : http://journals.openedition.org/anatoliaantiqua/657 ; DOI : https://doi.org/ 10.4000/anatoliaantiqua.657 


\section{ANATOLIA ANTIQUA ESKI ANADOLU}

\section{XXVI}

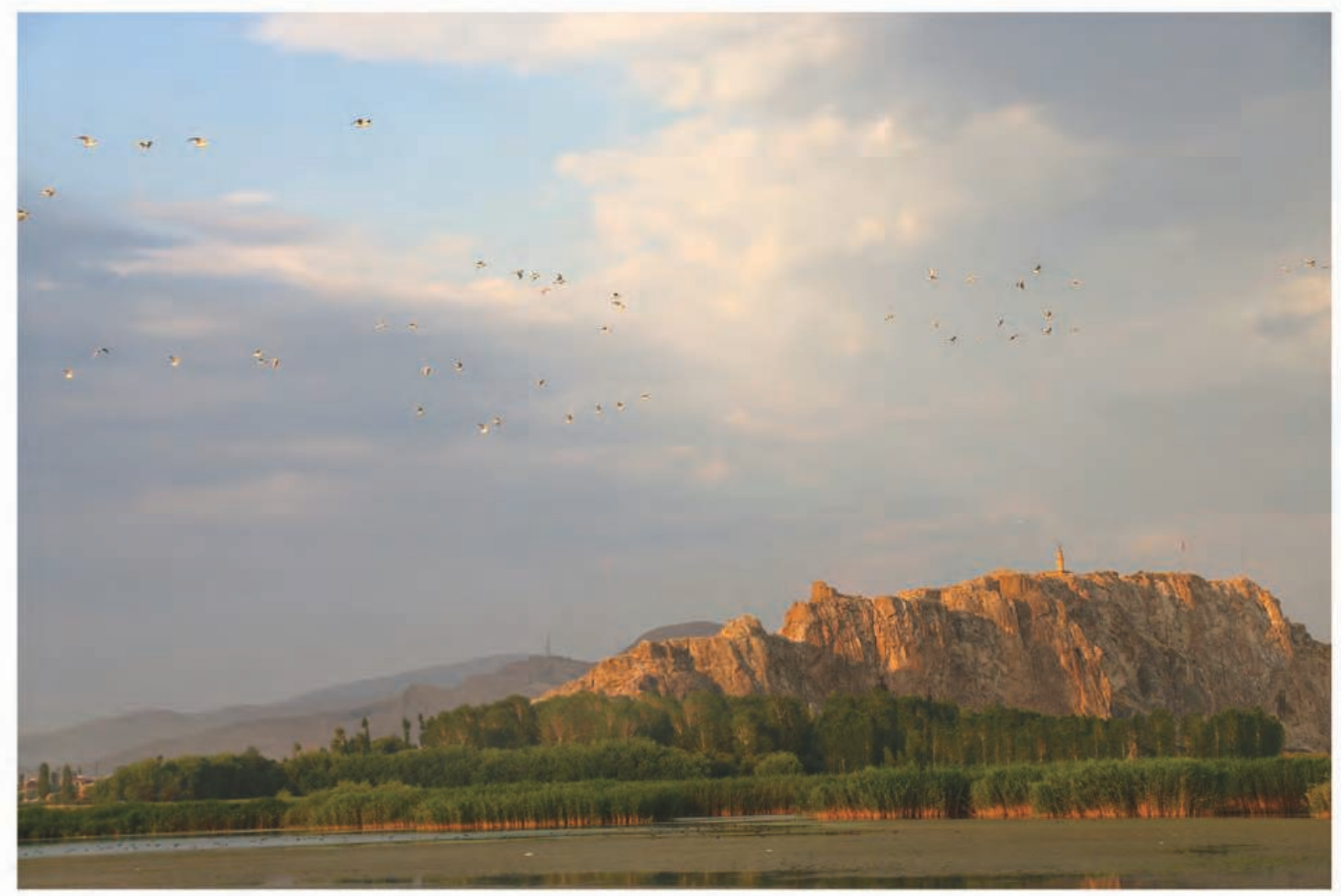

INSTITUT FRANÇAIS D'ETUDES ANATOLIENNES GEORGES-DUMEZIL

CNRS USR 3131

DE BOCCARD 


\section{TABLE DES MATIERES}

Alice VINET et Denis GUILBEAU

A First Glimpse of the Late Neolithic and Early Chalcolithic in Cappadocia through the Lithic

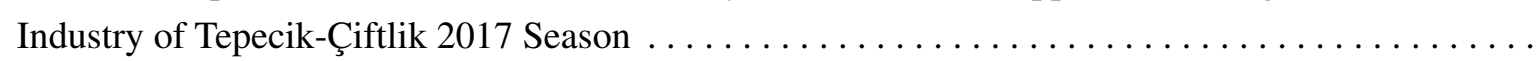

Ergül KODAŞ, Haluk SAĞLAMTIMUR et Yılmaz Selim ERDAL

Three Human Graves of the Hassuna Culture in Türbe Höyük

\section{Kevin PARACHAUD}

Les Galates en Asie Mineure au regard de la culture matérielle. Hellénisation, maintien, acculturation ? 23

H. Asena KIZILARSLANOĞLU et Erkan ALKAÇ

Hellenistic Amphora Stamps from Elaiussa . . . . . . . . . . . . . . . . . . . . .

Aygün EKİN MERİÇ

Late Roman Pottery from the Theatre of Nicaea in Bithynia $\ldots \ldots \ldots \ldots \ldots \ldots \ldots \ldots \ldots$

Nergis GÜNSENIN

La typologie des amphores Günsenin. Une mise au point nouvelle.

Nergis GÜNSENIN et Alessandra RICCI

Les amphores Günsenin IV à Küçükyalı (Istanbul). Un voyage entre monastères ? . . . . . . . . .

\section{CHRONIQUES DES TRAVAUX ARCHEOLOGIQUES EN TURQUIE 2017}

Erkan KONYAR, Bülent GENÇ, H. Banu KONYAR, Armağan TAN et Can AVCI

Excavations at the Old City, Fortress, and Mound of Van: Work in 2017 . . . . . . . . . . . . . .

\section{Çiğdem MANER}

Preliminary Report on the Fifth Season of the Konya Ereğli, Karapınar, Halkapınar and

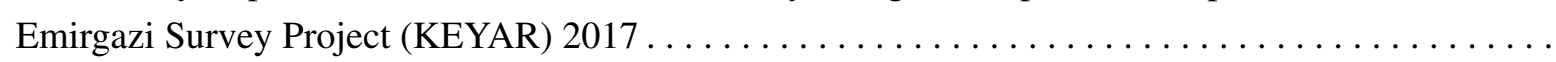

Abuzer KIZIL, Koray KONUK, Taylan DOĞAN, Didier LAROCHE, Enora LE QUERE,

Vasilica LUNGU, Francis PROST et Baptiste VERGNAUD

Eurômos : Rapport préliminaire sur les travaux réalisés en 2017

Olivier HENRY et E. ANDERSSON, J. BLID, Chr. BOST, Ö. ÇAKMAKLI, N. CARLESS-UNWIN, G. ÇİMEN, A. EYİGÖR, A. FRECCERO, A. FREJMAN, Cr. GEORGESCU, E. GOUSSARD, A.-M. GUIMIER-SORBETS, M. HAUCHART, R. HEDLUNG, N. LAMARE, V. LUNGU, Fr. MARCHAND-BEAULIEU, A. SITZ, I. STOJANOVIC, B. VERGNAUD 


\section{Olivier HENRY* et \\ E. ANDERSSON, J. BLID, Chr. BOST, Ö. ÇAKMAKLI, N. CARLESS-UNWIN, G. ÇİMEN, A. EYİGÖR, A. FRECCERO, A. FREJMAN, Cr. GEORGESCU, E. GOUSSARD, A.-M. GUIMIER-SORBETS, M. HAUCHART, R. HEDLUNG, N. LAMARE, V. LUNGU, Fr. MARCHAND-BEAULIEU, A. SITZ, I. STOJANOVIC, B. VERGNAUD}

\section{LABRAUNDA 2017}

La mission 2017 de Labraunda a été double. Parallèlement à la mission de fouille/documentation/conservation qui s'est déroulée sur le site de Labraunda du 18 juin au 18 août 2017, nous avons inauguré cette année une mission de prospection, pour l'instant modeste, mais qui s'avère très prometteuse. Cette dernière, dont la première édition s'est déroulée pendant les deux semaines qui ont précédé la fouille, a pour objectif d'établir une carte archéologique de la région de Labraunda et de permettre ainsi une meilleure compréhension du contexte de l'implantation de l'occupation à Labraunda. La zone concernée forme un cercle d'une vingtaine de $\mathrm{km}$ de diamètre autour de Labraunda.

Cette année, ce ne sont pas moins de 12 sites archéologiques qui ont été découverts par notre équipe. Des sites qui s'avèrent extrêmement variés typologiquement autant que chronologiquement.

A Labraunda même, suite à plusieurs années pendant lesquelles les fouilles ont été particulièrement intenses, cette année a été principalement consacrée à la documentation. Céramique, TCA, marbre, architecture, carpologie, faune sont autant de domaines qui ont été l'objet d'études à la fois ponctuelles et globales. A cette égard, nous tenons à souligner le lancement d'un nouveau domaine dans cette longue liste, puisque nous avons entrepris en 2017 une étude ambitieuse qui porte sur l'ensemble du matériel métallique mis au jour sur le site depuis les premières années de fouille en 1948. Cette étude est aussi l'occasion de faire un état des lieux sanitaires des centaines d'objets qui n'ont souvent reçu aucune attention depuis leur mise au jour. Ainsi, dès 2018, les travaux de conservations incluront une équipe dédiée à la conservation des objets en métal (bronze, argent, fer, et diverses alliages).

Les travaux de conservation/restauration/protection n'ont pas été en reste au cours de la saison. Le programme de protection des marbres s'est poursuivi, pour la cinquième année consécutives. A l'image de ce qui avait été fait l'année dernière pour les sols des Oikoi et de l'église est, les quelques 150 $\mathrm{m}^{2}$ de l'Andrôn ont fait l'objet d'un même traitement (géotextile et gravier) qui permet une protection particulièrement efficace des vestiges du sol original. Le point d'orgue du programme de restauration 2017 restera sans aucun doute les travaux entrepris à la "maison dorique". Cette fontaine monumentale datant de la période hékatomnide, réaménagée au cours du $1^{\text {er }}$ siècle ap. J.-C., avait été fouillée dans les années 1950, puis partiellement remblayée malgré un très beau sol mis au jour dans le bassin. Cette année a vu un programme ambitieux de fouille, visant à remettre au jour l'ensemble de la structure, suivi d'une restauration (laborieuse) de ses mosaïques de marbre et TCA.

Les travaux de fouille proprement dits ont concerné cinq secteurs différents. Il s'agissait de poursuivre notamment l'étude des installations de production (presse à huile et à vin) découvertes aux alentours du centre monumental. Ainsi deux presses ont été fouillées dans le voisinage de la tombe monumentale, tandis qu'une troisième a été dégagée (partiellement) dans la zone occidentale du territoire de Labraunda (presse "Persson"). Un nouveau programme de fouille a débuté sur la terrasse du temple avec le dégagement de la partie orientale de la stoa nord. Il s'agit, avec la stoa est (qui a fait

*) O. Henry, PSL* (ENS-AOrOc UMR 8546) et IFEA ; J. Blid, Université de Stockholm ; Chr. Bost, EPHE - ENS AOrOc UMR8546 ; Ö. Çakmaklı, Université de Karabük ; N. Carless-Unwin, Université de Warwick ; G. Çimen, Université de Uppsala ; A. Eyigör, Istanbul Restorasyon ve Konservasyon Merkez Laboratuvarı ; A. Frejman, Université de Uppsala ; Cr. Georgescu, Institut d'Archéologie Vasile Pârvan de Bucarest ; E. Goussard, PSL* (ENS-AOrOc UMR 8546) ; A.-M. Guimier-Sorbets, ArScAn, UMR 7041 ; M. Hauchart, Paléotime; R. Hedlung, Université d’Uppsala ; N. Lamare, CNRS UMR8167 ; V. Lungu, Institute of SouthEastern European Studies, Bucarest ; Fr. Marchand-Beaulieu, PSL* (ENS-AOrOc UMR 8546) ; A. Sitz, Harvard University; I. Stojanovic, Institut d'Archéologie Belgrade ; B. Vergnaud, IFEA. 
l'objet cette année d'une documentation préparatoire à un programme de fouille) du dernier bâtiment hékatomnide connu et non encore étudié dans son intégralité. Enfin, les travaux de dégagement de l'impressionnant bassin romain, situé au pied du site, le long de la route moderne, ont pu reprendre après une saison de pause.

L'ensemble des travaux mentionnés ci-dessus n'auraient pu avoir lieu sans la formation d'une équipe nombreuse et entièrement dédiée au site, d'une part, et l'implication de partenaires scienti- fiques et financiers, d'autre part. Que tous soient ici chaleureusement remerciés.

La rédaction de ce rapport est le fruit d'un travail collaboratif dans lequel chaque responsable de projet est intervenu. C'est la raison pour laquelle certaines parties sont rédigées en anglais. La longueur particulièrement inhabituelle de ce rapport s'explique par la "double mission" de 2017 comprenant à la fois une prospection étendue et une mission de fouille, documentation, analyse, restauration et protection.

\section{EQUIPES ET PUBLICATIONS}

\subsection{LES EQUIPES DE LA MISSION 2017}

L'année 2017 est marquée par la création d'une mission "annexe" qui permet de compléter la fouille de Labraunda par un grand projet de prospection dans la région. A cet égard, deux équipes se sont succédées sur le terrain. La première, modeste, est intervenue pendant deux semaines, du 4 au 17 juin 2017, tandis que la seconde, beaucoup plus importante, a travaillé du 18 juin au 18 août 2017.

La première équipe, de prospection, était composée des membres suivants :

1. HENRY Olivier : directeur de la mission, archéologue, chercheur associé UMR AorOc-ENS (UMR 8546) :

2. EYİGÖR Ayşe : archéologue, conservatrice au centre national de conservation à Istanbul :

3. MARCHAND-BEAULIEU Frédérique : ingénieur d'étude à l'UMR AorOc-ENS (UMR 8546);

4. GEORGESCU Cristina : conservatrice à l'institut national d'archéologie de Bucarest ;

5. HENRY Sébastien.

La seconde équipe, de fouille, était composée de 42 membres (dont 1/3 d'étudiants de licence), originaires de 12 nationalités différentes, représentant 28 institutions internationales dont 5 universités turques :

1. ANDERSSON Erika, Uppsala Univ.

2. ARDIL Cem, Mimar Sinan Univ.

3. ARSLAN Anıl, Aydın Univ.

4. BLID Jesper, Stockholm Univ.

5. BOLAT Özlem, Karabük Univ.

6. BOST Christophe, IFEA

7. CARLESS Naomi, Warwick Univ.

8. ÇAKMAKLI Ömür, Karabük Univ.

9. CELIK Güney, Mimar Sinan Univ.
10. CHARLIER Fabrice, Archeodunum

11. ÇİMEN Görkem, Uppsala Univ.

12. CIUBOTARU Alina, Bucarest Univ.

13. DURAK Çağla, Bilkent Univ.

14. FREBAULT Eloïse, ENS Paris

15. FRECCERO Agneta,

16. FREJMAN Axel, Uppsala Univ.

17. GEORGESCU Cristina, Institut national

d'archéologie de Bucarest

18. GOUSSARD Elisabeth, ENS Paris

19. GÜNAL Merve, Bilkent Univ.

20. HAUCHART Mélanie, Montpellier Univ.

21. HEDLUND Ragnar, Uppsala Univ.

22. HELLSTRÖM Pontus, Stockholm Univ.

23. HENRY Olivier, IFEA-ENS

24. HOLICKOVA Sona, Musée national de

Danemark

25. IANCU Liviu, Bucarest Univ.

26. KAPLANSEREN Onurcan, Karabük Univ.

27. KRANIG Friederike, Ludwig Maximilians Univ.

28. LAMARE Nicolas, Picardie Univ.

29. LAURENT Anne Sophie, Paris 1 Univ.

30. LUNGU Vasilicia, Institut national d'archéologie de Bucarest

31. MANEA Ioana, Bucarest Univ.

32. MARCHAND-BEAULIEU Frédérique,

ENS Paris

33. MEREY Deniz, Bilkent Univ.

34. POPOVA Tzvetana, Sofia Univ.

35. RADON Jenny,

36. SARGÜNEY Güneş, Mimar Sinan Univ.

37. SENER Kadir, Karabük Univ.

38. SITZ Anna, U Penn

39. STOJANOVITC Ivana, Belgrad Univ.

40. STREINU Alina, Musée de Bucarest

41. VATANSEREN Sanem, Karabük Univ.

42. VERGNAUD Baptiste, IFEA 


\subsection{PUBLICATIONS ET CONFÉRENCES AUTOMNE 2016 / AUTOMNE 2017}

\section{Conférences}

10/2017 : Cristina Georgescu, “Archaeological sites and attitudes towards conservation. Labraunda, Turkey", The National Symposium Vasile Pârvan.

08/2017: Baptiste Vergnaud, "Labraunda, Eurômos and the fortifications of Inland Caria", Euromos Excavation House.

05/2017: Baptiste Vergnaud, "The fortifications of Labraunda: military architecture and daily life in Classical and Hellenistic Caria", IFEA, Istanbul.

04/2017 : Vasilica Lungu, "Un thymiaterion din necropola de la Labraunda. Relaţia dintre obiectul ceramic şi monumentul funerar: fantezie sau reflex al istoriei locale? (Un thymiaterion de la nécropole de Labraunda. Relation entre l'objet céramique et le monument funéraire : fantaisie ou reflet de l'histoire locale)", Institut d'Archéologie "Vasile Pârvan", Bucarest.

03/2017 : Olivier Henry, "Sanctuaire et pouvoir. Nouvelles pistes de réflexion à partir des recherches archéologiques récentes sur le site de Labraunda en Carie", Académie des Inscriptions et Belles Lettres, Paris.

03/2017 : Olivier Henry, "Un personnage fragmenté. La figure d'Hekatomnos, satrape de l'Empire perse et dynaste carien", ENS, Paris.

01/2017 : Olivier Henry, "L'expression du pouvoir de Mausole et le sanctuaire de Labraunda en Carie", SFAC, Paris.

01/2017 : Axel Frejman, "Labraunda, de senaste årens fältarbeten", Mediterranean museum in Stockholm.

11/2016 : Olivier Henry, "The more we learn the less we know? A reappraisal of Labraunda's last excavation seasons", Université d'Uppsala.

\section{Contributions dans des colloques internationaux}

09/2017 : Pontus Hellström, “Achaemenid Presence or Influence at Labraunda? The Relief Sculpture of a Chariot", Achaemenid Anatolia: Persian Presence and Influence in the Western Satrapies 546-330 BC, Swedish Research Institute at Istanbul.

09/2017 : Jesper Blid, "The Andrôn of Maussollos at Labraunda and its architectural sculpture. Aesthetic fluidity and cultural allegory in Hekatomnid Karia", Achaemenid Anatolia: Persian Presence and Influence in the Western Satrapies 546-330 BC, Swedish Research Institute at Istanbul.
09/2017 : Olivier Henry, "From a Local Karian Sanctuary to a Topos of Persian Royal Display: New Thoughts on Labraunda in Karia", Achaemenid Anatolia: Persian Presence and Influence in the Western Satrapies 546-330 BC, Swedish Research Institute at Istanbul.

08/2017 : Olivier Henry, "Labraunda 2017 Yüzey Araştırması", 10. Karia/Karialılar ve Mylasa Sempozyumu, Milas (Turquie)

05/2017 : Olivier Henry, "Labraunda 2016", Kazı Sonuçları Toplantısı, Bursa (Turquie).

\section{Publications}

Ö. Çakmaklı, "Labraunda Su Kompleksi Kazıları ve İnce Cidarlı Seramiği ( Ön Rapor)/ The Thin Walled Pottery from the Excavation at Labraunda Water Complex (Preliminary Report)", Pamukkale University Journal of Social Sciences Institute 28, 2017 : 257-269.

Ö. Çakmaklı, "Zeus Labraundos Kutsal Alanı Su Kompleksi Kazıları Cam Buluntuları / Glass Finds from Water Complex in the Sanctuary of Zeus Labraundos", Seleucia VII, 2017 : 279-297.

Ö. Çakmaklı, "Labraunda'nın Yeni Bilmecesi: Su Kompleksi Kazıları 2013-2015 Kazı Sezonu Değerlendirmesi / The Mystery of Labraunda: Water Complex Excavations", Journal of History Culture and Art Research 5.3, September 2016 : 15-25.

A. Frejman, "En måndag vid Zeus helgedom", Nya Populär Arkeologi 1, 2016 : 30-31.

O. Henry, "Quel(s) portrait(s) pour les Hékatomnides ?", in D. Boschung et Fr. Queyrel (éds.), Bilder der Macht, Das griechische Porträt und seine Verwendung in der antiken Welt, (Morphomata, Band 34), Leiden 2017 : 101-119.

O. Henry, "Hekatomnus, Son of Hyssaldomus: A Unicum in Persian History", in K. Iren et al. (éds.), The Persians: Power and Glory in Anatolia, Istanbul 2017 : 350-365.

O. Henry et al., "Labraunda 2016", Anatolia Antiqua XXV, 2017 : 187-266.

V. Lungu, "Un thymiaterion à décor floral de Labraunda ?", Bollettino dell'Associazione Iasos di Caria 23, 2017 : 44-49.

\section{Doctorat et Master (soutenus en 2017)}

Anna Sitz, "Labraunda", in: The Writing on the Wall: Inscriptions and Memory in the Temples of Late Antique Greece and Asia Minor, $\mathrm{PhD}$ dissertation, University of Pennsylvania, 2017 : 170-188.

Görkem Çimen, The excavations of the P-building and the R-bath at Labraunda: Archaeology in 
the 1950s based on Inge Dahlén's three excavation diaries, B.A. thesis, Uppsala University, 2017.

\section{Workshops/Labraunda Day}

\section{5-16 novembre 2016, à l'Université d'Uppsala} (Suède)

15 Novembre

13:15 Axel Frejman, The surroundings of Labraunda

14:15 Lars Karlsson, The defense network at Labraunda

14:45 Christophe Bost, The East Bath

15:30 Olivier Henry, Toughts on the developments of activities at Labraunda and how to synchronize excavation with conservation/preservation and presentation to the public?

16:00 Pontus Hellström, Terrace House II at Labraunda and the rusticated wall

16:30 Jesper Blid / Ragnar Hedlund, The Roman buildings on the $M, B$ and $Y$ terraces

17:00 Vasilica Lungu, Methodological approach on ceramic material at Labraunda

16 Novembre

9:15 Nadine Schibille, Glass supply and working at Labraunda

9:45 Ömür Çakmaklı, Glass typological studies, a methodology

10:15 Naomi Carless-Unwin, Epigraphy at Labraunda, future projects

10:45 Agneta Freccero, Marbles and Stucco braunda

11:25 Discussion on plans for future projects at La-

13:30 The course of future work at Labraunda

15:15 Lecture by Excavation Director Olivier Henry

17:00 Book release by Jesper Blid, Labraunda 4, Remains of Late Antiquity.
7 février 2017, au Swedish Research Institute at Istanbul, en collaboration avec l'IFEA

14:30 Doç. Dr. O. C. Henry, Introduction

The Surroundings of Labraunda

14:50 A. Frejman, Strabo says Labraunda is a village, what does the archaeology say?

15:10 B. Vergnaud, The Towers in the Fortifications of Inland Caria in the Classical and the Hellenistic Period

Material Studies

15:30 V. Lungu, Ceramics from the Nekropolis, an overview on typology and chronology

15:50 Ö. Çakmakl1, The Glass finds from Water Pools and The General View of The Labraunda Glass

The East Baths Complex

16:30 Chr. Bost / A. Henry, An appraisal on three excavation seasons at the East Baths

16:50 A. Henry, New considerations on the "East Church"

17:10 O. Henry, Conclusion, future research projects at Labraunda

17:30 Discussion

18:00 Book presentation by J. Blid (Stockholm Univ.) Remains of Late Antiquity

\section{Exposition}

12/2016 : Exposition virtuelle Zeus à la double hache le sanctuaire de Labraunda (https://explore. univ-psl.fr/fr/exposition-virtuelle/zeus-\%C3\%A0la-double-hache-le-sanctuaire-de-labraunda)

\section{PROSPECTION}

La mission Labraunda de 2017 a débuté par une large prospection qui s'est déroulée sur deux semaines, du 4 au 16 juin 2017.

Depuis le début des recherches menées sur le site de Labraunda, et particulièrement à la lumière des dernières découvertes, Labraunda apparaît comme un lieu clé de la compréhension de l'évolution des contextes historiques et culturels du SudOuest de l'Anatolie.

Labraunda fut longtemps considéré comme un îlot de civilisation perdu au centre d'un "no man's land" de verdure, sur les pentes marquées de la chaîne de montagnes du Latmos. Les recherches très récentes, menées à la fois au cœur du complexe architectural, ainsi que dans son voisinage immédiat, ont montré qu'il n'en était rien. Mais encore, jusqu'à récemment, il était généralement admis que l'histoire du site et de l'occupation de cette zone avait débuté dans le courant de la période archaïque, autour du $6^{\mathrm{e}} \mathrm{s}$. av. J.-C. Là encore, de récentes découvertes ont permis de remettre en cause cette idée reçue en démontrant que l'occupation avait débuté probablement dès la fin du Chalcolithique ou le tout début de l'Age du Bronze ${ }^{1}$.

Le même 'bond chronologique' fut opéré il y a quelques années dans la partie nord du Latmos, grâce aux recherches dirigées par A. Peschlow. Ces dernières ont démontré l'existence de communautés organisées dès le milieu de la période chalcolithique autour du lac de Bafa, à une trentaine de kilomètres au nord de Labraunda ${ }^{2}$.

1) Voir Henry et al. 2013 : 298-300.

2) Peschlow-Bindokat et Gerber 2012 
L'objectif des recherches que nous avons décidé d'entreprendre en 2017 est de fournir une carte archéologique des environs de Labraunda, à grande échelle. Il s'agit de procéder à un inventaire précis des vestiges situés dans un rayon de plusieurs kilomètres autour du site. La mise en place de cette carte archéologique permettra de préciser la chronologie et les conditions d'implantation de communautés dans cette région de la Carie. La zone qui nous intéresse (d'une superficie totale de 180 $\mathrm{km}^{2}$ ) est pour l'instant une véritable terra incognita puisqu' aucune recherche de ce type n'y a encore été menée.

\subsection{REMARQUES PRELIMINAIRES}

\section{a. Durée du projet}

Le projet est planifié pour durer au moins 4 années. Compte tenu du peu d'informations dont nous disposons à l'heure actuelle sur la région il est toutefois possible que cette période soit reconsidérée, en cas de découvertes majeures.

La zone concernée couvre l'intégralité de la région qui ceinture le site de Labraunda (Fig. 2.1.1). Elle s'étend sur $15 \mathrm{~km}$ du Nord-Est au Sud-Ouest et environ $12 \mathrm{~km}$ du Nord-Ouest au Sud-Est. Elle s'adapte à la topographie locale tant que faire se peut ; c'est-à-dire qu'elle inclut des massifs entiers de la chaîne montagneuse et trouve ses frontières en fond de vallée ou au niveau des crêtes montagneuses.

La zone a été divisée en quatre portions : est, nord, ouest et sud. Chacun de ces quadrants fera l'objet d'une saison de recherche. La saison 2017 a été dédiée au quadrant ouest.

\section{b. Principes de la prospection}

Compte tenu de la topographie très marquée, nous avons décidé d'adopter une approche extensive plutôt que systématique. Dans un premier temps il s'agissait de repérer des zones d'intérêt à partir d'images satellite (QuickBird), de procéder à une analyse de la toponymie sur les cartes $1 / 25000^{\mathrm{e}}$, et de recenser les découvertes fortuites enregistrées auprès de la commission des monuments historiques de la région de Muğla. Ces trois éléments fournissent une première trame de recherche que nous avons enrichie à partir d'une enquête locale menées auprès des habitants des villages monticoles.

\subsection{TECHNIQUES DE DOCUMENTATION}

Tout les vestiges rencontrés ont été documentés aussi précisément que possible. Cette documentation inclut : une prise de coordonnées GPS ; une couverture photographique; des relevés dessinés au $1 / 20^{\mathrm{e}}$ (pour la plupart des vestiges construits) et parfois à l'échelle 1 . Lorsque les vestiges étaient trop étendus pour pouvoir être documentés à la main, nous avons procédé à un relevé topographique associé à un relevé photogrammétrique (sous la responsabilité de Fr. Marchand-Beaulieu), lequel a pour objet d'offrir une modélisation tridimensionnelle des vestiges concernés.

Cette technique de documentation a été particulièrement utile dans le cas des abris sous roche, dont les nombreuses aspérités sont particulièrement difficiles à relever. Afin de réaliser les modèles photogrammétriques, plusieurs séries de photographies à pied ont été prises pour chaque abri, avec des photos de détail pour les peintures. L'appareil photo est un Nikon D80, avec un objectif DX 18-70 mm. Au vu de la complexité de certaines structures, des relevés topographiques agrémentés de cibles installées sur les parois ont été nécessaires. La station Leica utilisée est une TS15. Lorsque le relevé topographique par tachéomètre laser n'était pas techniquement possible, nous avons pris des mesures entre les cibles afin de vérifier les modèles et de les modifier le cas échéant (SAR 007 et 008). Ces modèles serviront à présenter en 3D les abris relevés et sont également une base de travail sur l'ensemble du support pour comprendre, entre autre, l'occupation spatiale des rochers ou encore les schémas globaux du programme pictural.

\subsection{LES LIMITES DE LA PROSPECTION}

\section{a. La topographie}

Comme indiqué plus haut, la topographie est extrêmement marquée dans la région. C'est pourquoi nous ne pouvions pas procéder à une enquête systématique. S'appuyant principalement sur les informations fournies par les habitants locaux, les résultats de cette année ne reflètent que l'état de nos connaissances et non pas une carte archéologique exhaustive. Une autre conséquence du faciès topographique est que notre enquête couvrait essentiellement les zones habitées et les "yaylas" (estives), c'est-à-dire là où les gens connaissent le mieux leurs terres. Par conséquent, notre recherche fut circonscrite dans une bande de terre comprise entre 350 et $700 \mathrm{~m}$ d'altitude. Néanmoins, comme le montre les résultats glanés cette année, la mission 2017 a été particulièrement gratifiante avec 


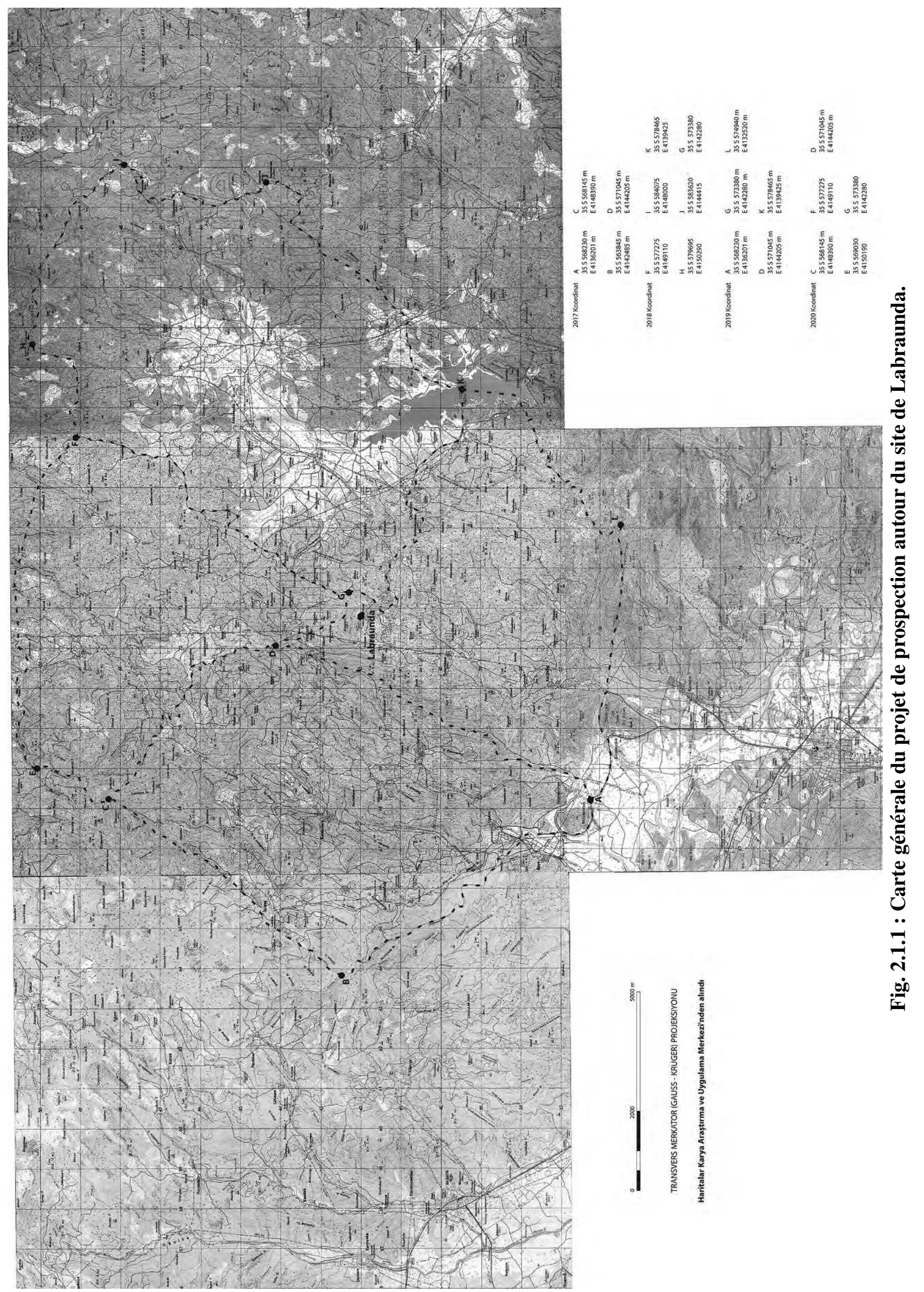




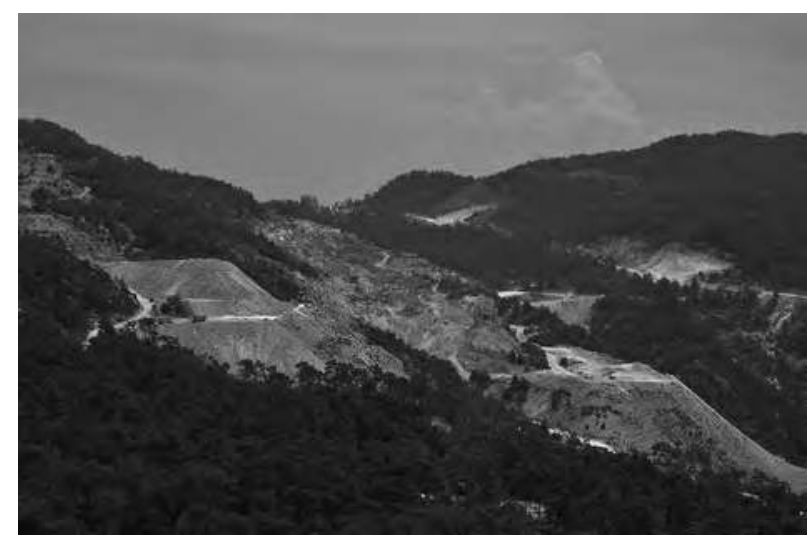

Fig. 2.3.1 : La carrière de feldspath au Nord d'Asarlık tepe (O. Henry).

12 sites découverts et documentés couvrant une vaste période depuis le Chalcolithique jusqu'à l'ère byzantine. Il est prévu de revenir sur cette même zone en 2018, avec des enquêtes test à la fois dans les secteurs élevés de la région, autour des pentes d'Oynalan $(1081 \mathrm{~m})$ et de Turgut tepe $(1119 \mathrm{~m})$ à l'Est, ainsi que autour de Çeyizkaya $(1181 \mathrm{~m})$ à l'Ouest, et dans les vallées basses autour des villages de Çomakdağ, Çınarlı et Bahçeburun.

\section{b. Les activités minières}

La région de Latmos est aujourd'hui célèbre pour ses ressources en feldspath. Les carrières sont florissantes dans la région et couvrent malheureusement de très grandes surfaces sur le versant sud du mont Latmos. Dans la zone couverte par la mission de 2017, nous avons compté au moins 10 carrières différentes, certaines modestes, d'autres très grandes (Fig. 2.3.1). Ces carrières posent deux types de problèmes différents. Le premier problème est la destruction systématique non seulement de la flore et de la faune, mais aussi des vestiges archéologiques. Bien que les autorisations d'exploitation ne soient données qu'après une enquête menée par les autorités locales, l'expérience a montré qu'il est presque impossible de mener une recherche systématique impliquant une topographie si difficile. Le deuxième problème résultant de cette activité concerne l'accès à différentes zones du secteur. En raison des lourds camions chargés de feldspath, non seulement les anciennes routes sont complètement détruites, mais les nouvelles routes sont aussi particulièrement dangereuses. La création continue de ces

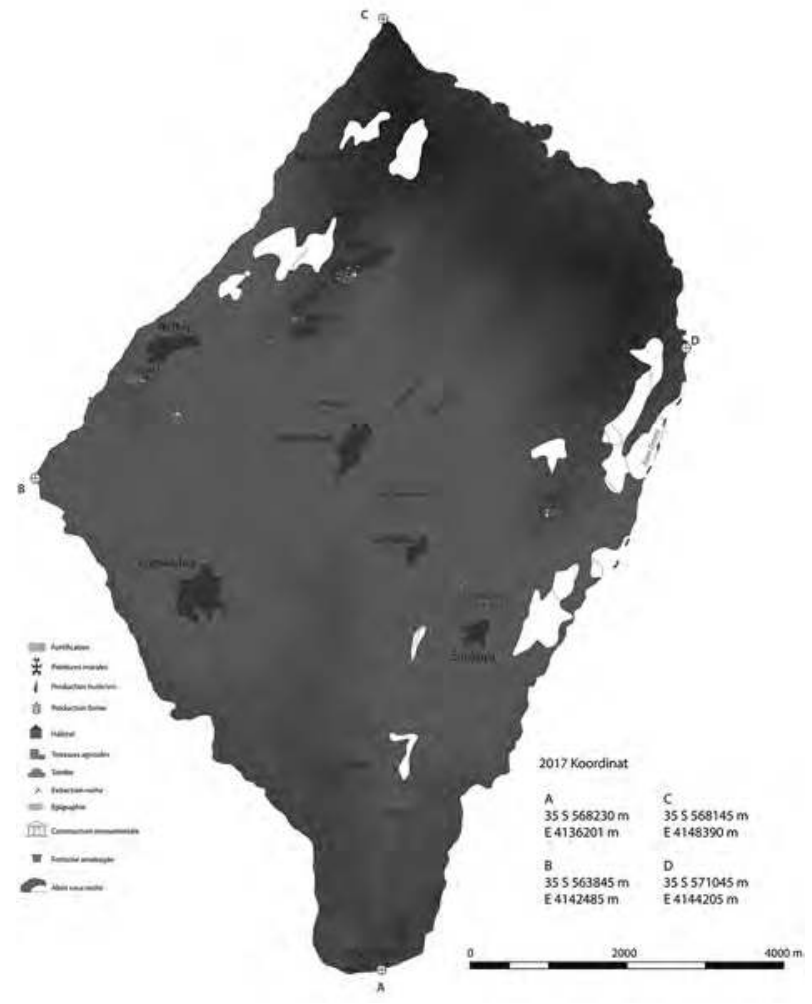

Fig. 2.4.1 : Carte de localisation des sites découverts au cours de la prospection 2017

(O. Henry).

dernières pose un problème de sécurité tout autant que de navigation dans cette région très montagneuse puisque nous sommes amenés à nous appuyer sur des cartes qui ne reflètent pas le dernier état des routes.

\subsection{LES RESULTATS 2017}

La superficie totale qui a été incluse dans le permis de cette année comprenait, du nord au sud, les villages de İkiztaş, Ketendere, Çomakdağ, Sarıkaya, Çınarlı et Bahçeburun. En raison de la quantité impressionnante de vestiges découverts au cours de l'enquête, nous n'avons malheureusement pas pu couvrir ni le sud ni le nord de la région. Au total, 12 sites différents ont néanmoins été découverts (Fig. 2.4.1).

\subsubsection{SARIKAYA}

\section{A. Les peintures rupestres (par A. Eyigör)}

So far, almost 200 rock paintings have been found in numerous rock shelters and caves in the Latmos range since $1994^{3}$. These paintings were

3) Peschlow-Bindokat 2014: 22. 


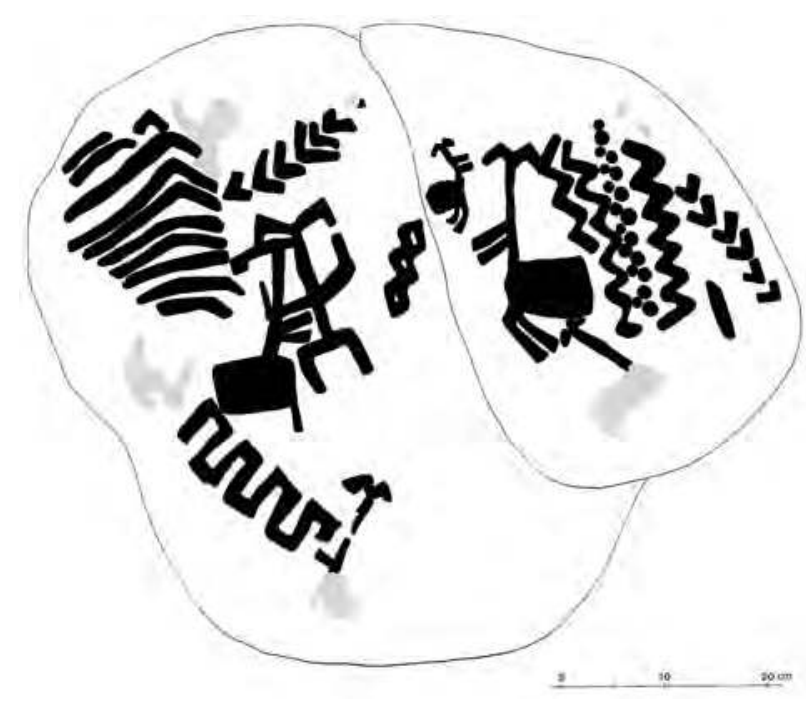

Fig. 2.4.2 : Copy of paintings in two niches of Balıktaş-5 (Peschlow-Bindokat 2014 : 62).

mostly composed of red ochre and, more rarely, yellow ochre and white. Most of the paintings represent individuals: women are depicted with steatopygia in profile and men are depicted with rised arms and open legs in front view (Fig. 2.4.2). They both have either $M$ shaped heads in schematic style or round head in naturalistic style. The individuals (whether human or animal) can be shown as single, in couple or grouped. Also, among the representations one can notice the presence of some geometric signs like zigzags, lines, dots, squares or meanders.

The rock paintings are dated so far by A. Peschlow to the Late Neolithic and Early Chalcolithic periods, which are defined for western Anatolia in the $6^{\text {th }}$ and $5^{\text {th }}$ millenium $\mathrm{BC}$, in regard to a sondage excavation in the Malkaya cave and some surface findings. Also, some patterns on women's dresses in paintings have similarity with ceramic patterns found on the Hacılar and Kuruçay ceramics ${ }^{4}$.

A new series of rock-cut paintings, similar to the ones found previously has been discovered during our survey around the village of Sarikaya (Milas, Muğla). In this village, 3 rock-paintings had already been found by A. Peschlow, although never published. Our survey, aiming at documenting the already known paintings led to the discovery of 9 more groups of paintings, as well as of 3 rock-shelters with pigment traces. Human/ animal figures and geometrical signs had been painted with red ochre and most of them are covering multi-faced rock-shelters. Besides, these

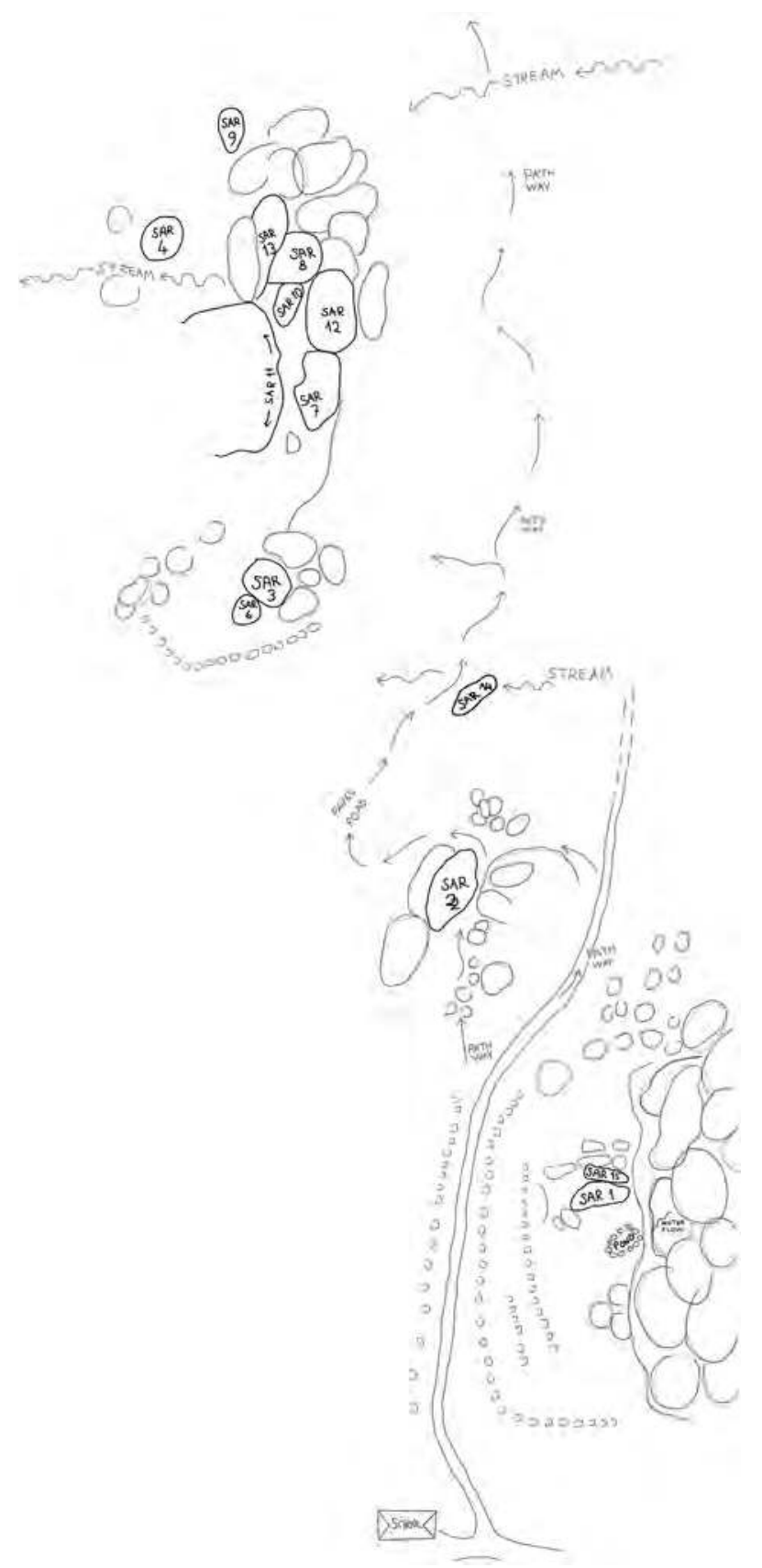

Fig. 2.4.3 : Outline drawing of the Sarıkaya Rock paintings (A. Eyigör).

paintings seem to be link to rock-cuttings of lines, holes and cupules in two different locations. The organization of the Sarıkaya rock-paintings seem to follow a pathway, leading from below the village northward, along the western slope of the hill (Fig. 2.4.3).

4) Peschlow-Bindokat 2012: 76. 


\section{A.1. Materials and Methods}

Beside the usual documentation techniques presented above, and specifically for analyzing the rock-paintings, we used a software $\left(\right.$ DStretch $\left.^{\circledR}\right)$ allowing us to enhance the digital photos and revealing better details of the paintings.

It quickly appeared that single boulders provided several shelters, and that each shelter carried a different group of paintings. Therefore, each boulder carrying painting(s) has been given a serial digit $(1,2,3$, etc.) and each face of the boulder carrying paintings has been attributed a specific letter $(\mathrm{A}, \mathrm{B}$, $\mathrm{C}$, etc.).

Most of the figures and signs are faded because of time and weathering. Only identifiable figures could be copied by wetting the surface with deionized water spray, then they were transferred on acetate films by drawing. This method can be used only on robust rock surfaces and stable pigments. When the surface will not allow a direct copy, documentation will be done through scaled and enhanced detail photographs. Because of limited time and difficulties in reaching some areas, only the three first boulder's paintings could be documented in details this year. We plan to proceed to the complete documenting of the other paintings in the coming years.

The study of these paintings will be integrated in a larger approach on prehistoric paintings of the Latmos, in the frame of the $\mathrm{PhD}$ thesis on prehistoric landscape archaeology and symbolism.

\section{A.2. Results}

\section{A.2.1. Sarlkaya-1}

Sarkaya-1 is the closest painting to the village, along the pathway (Fig. 2.4.4). It has two faces (North and South). Two figure groups on both face can be seen clearly from the pathway side (Fig. 2.4.5). Other figures and shapes are faded or covered with patina. Face A (north) contains 11 human figures, 2 geometric shapes, numerous dots and many unidentifiable shapes. A shape resembling a vulva is significant here (Fig. 2.4.6). Face B (south) (Fig. 2.4.7) has 4 human figures, geometric shapes with lines and zigzags. Here, one man has a W shaped head, a peculiarity that seems to be only found in our Sarıkaya group compared to other Latmos examples (Fig. 2.4.8). The same rock-face also carries a group of 2 lines and 2 zigzags vertically arranged in a deep niche.

The configuration of the rock creates natural passages on the sides and underneath the shelter
(Fig. 2.4.9), bringing to mind the possibility that this peculiar location might have been used for inititation rituals.

\section{A.2.2. Sarıkaya-2}

This rock shelter is located very close to and west of Sarıkaya-1, on the left side of the pathway where two ways merge on the side of the shelter and continue down to other rock paintings (Fig. 2.4.10). The boulder has an oval shape of a cocoon and there are 3 faces with paintings, respectively on the east, north and west faces. Face A (east) forms the side of a narrow corridor and carries three human figures, one hand, two geometric shapes, numerous dots and many unidentifiable shapes (Fig. 2.4.11). Face B (north) is a dome-shaped pocket with one single and one couple human figures, several dots and some faded shapes. One of the most interesting paintings in Sarıkaya is on Face C (west) (Fig. 2.4.12). This face is crowded with 1 human and 4 animals (at least), as well as a hand, lines, dots and many faded human figures. A caterpillar/larvae and 2 or 3 butterfly/moth/dragonfly can be seen on the right side (Fig. 2.4.13). This is the first time that caterpillar and butterflies are depicted among the Latmos rock-paintings. They could participate in the symbolizm of metamorphosis/(re)birth. The paintings also contain a sheep/goat in a square (fence?) with a man holding its neck, which might be a representation of the relationship between animal and human, and the domestication of the former.

Another distinctive feature of this group of paintings is the presence of paint remains on the floor, in the shape of a large dot and a long half-circle (Fig. 2.4.14). The big amorphous dot in the center could be a paint residue only. It could also indicate that the paint itself could have been prepared on the floor (in this specific location). The dot might also have been drawn deliberately in order to show the center of a half circle, remains of which can still be visible. This half-circle is particularly puzzling; it might very well be the materialization of some sort of specific/reserved/protected area. The dot is also located at the best location to be seated here. Other parts of the rock are very steep and slippery.

One last distinct feature of the boulder is the shape of its natural niches on the ceiling. Here, the natural shape of the niches have been highlighted with paint in order to complete what seems to represent a woman's silhouette (Fig. 2.4.15).

One must notice also that this location has one of the best view over the entire region. It is looking directly westward, toward the twin rocks of İkiztaş, 


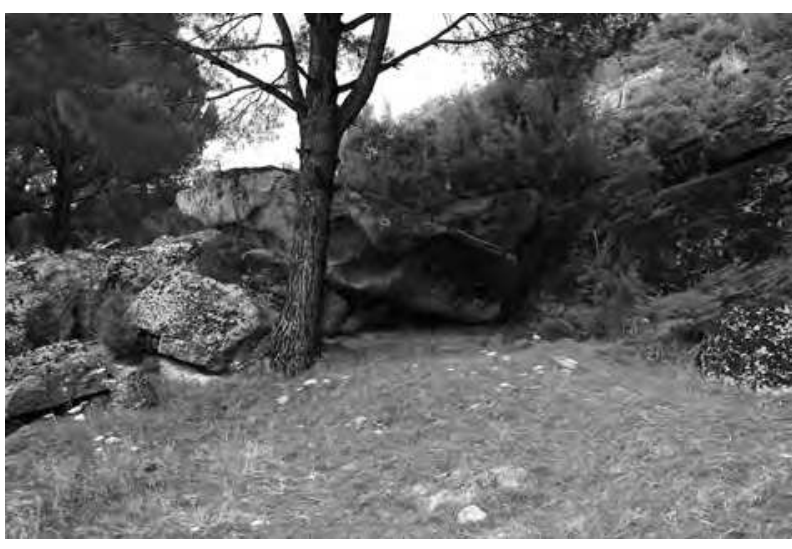

Fig. 2.4.4 : Sarıkaya-1 view from southwest (A. Eyigör).

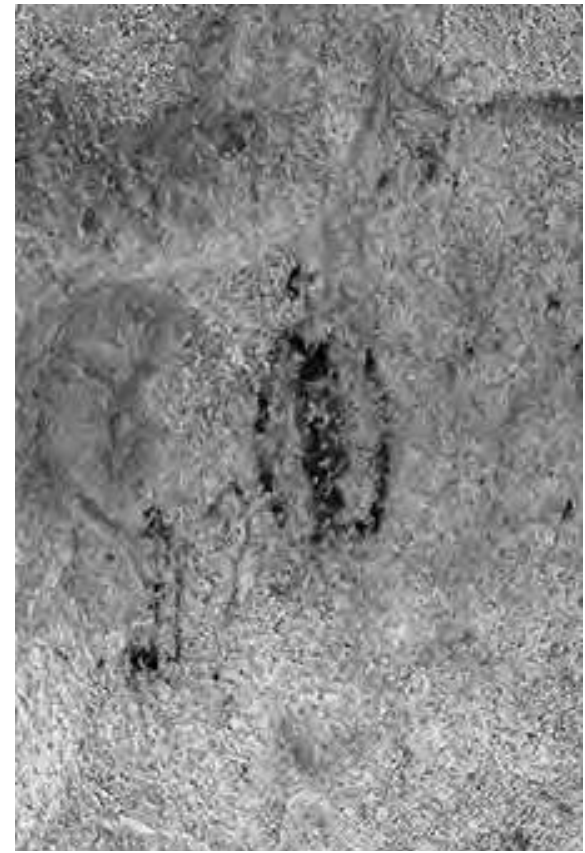

Fig. 2.4.6 : Enhanced detail from Sarkaya-1 Face $A$. In the middle, a vulva like shape and on the left below, a man/woman couple can be seen. There are also many paint traces, possibly remains of human figures (A. Eyigör).

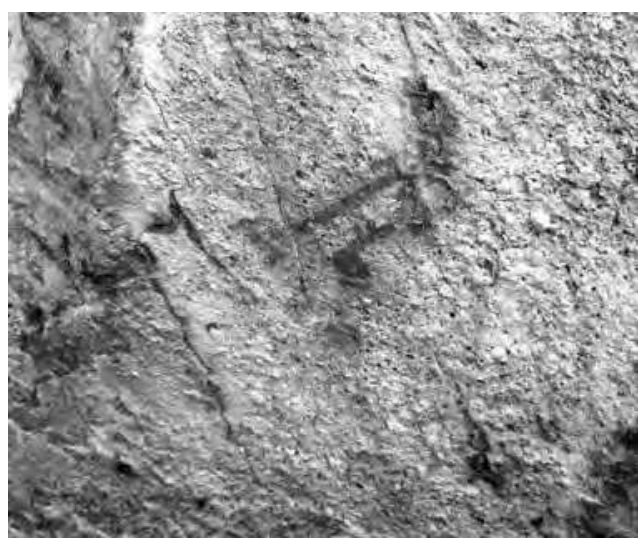

Fig. 2.4.5 : Figure group on right side of face A in Sarkkaya-1 (A. Eyigör).

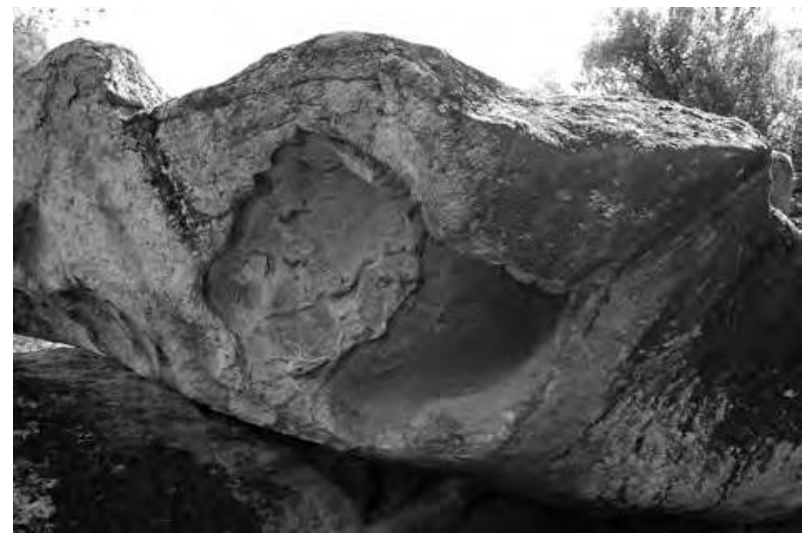

Fig. 2.4.7 : Face B (south) of Sarkaya-1 (A. Eyigör).

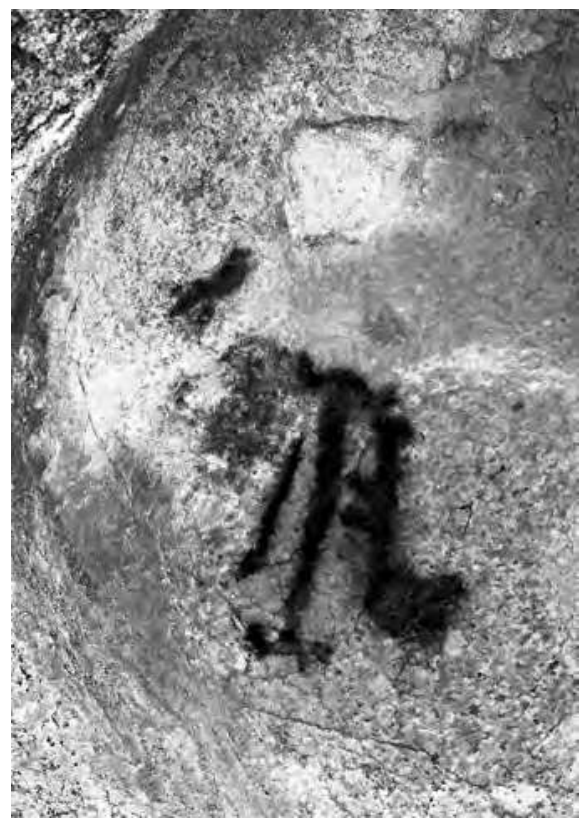

Fig. 2.4.8 : Enhanced detail of Face B (A. Eyigör). 


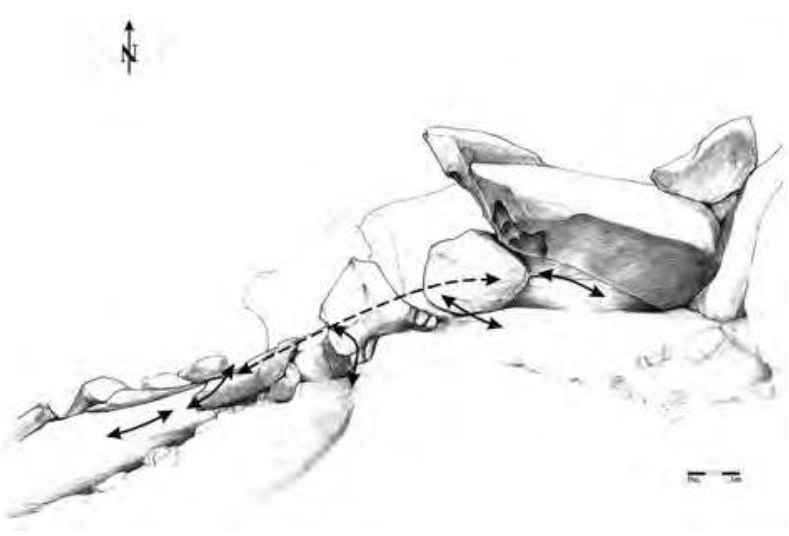

Fig. 2.4.9 : Scaled drawing of Sarkaya-1 from south. Passages are shown with arrows. There is a wide courtyard in three level and man-made pond in southern part of shelter (C. Georgescu).

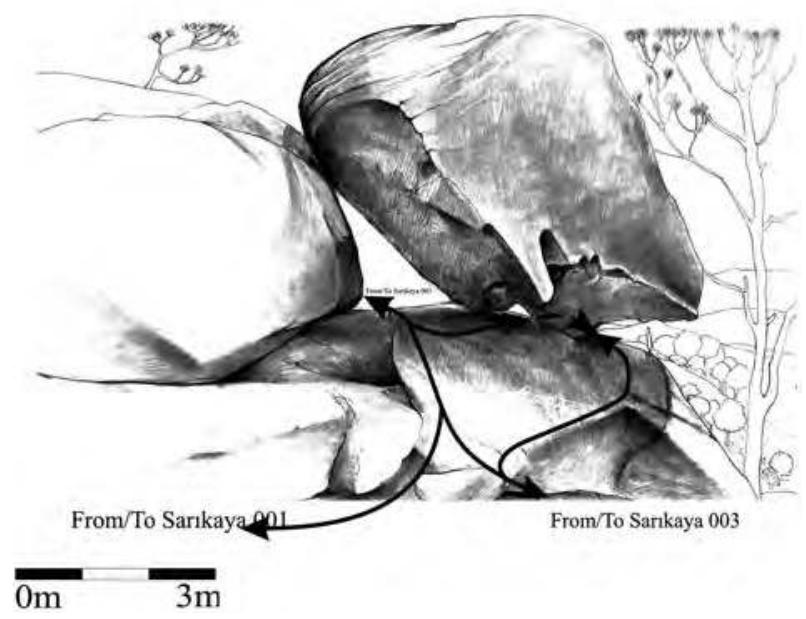

Fig. 2.4.10 : Scaled drawings of Sarkaya-3 showing the passages (C. Georgescu).

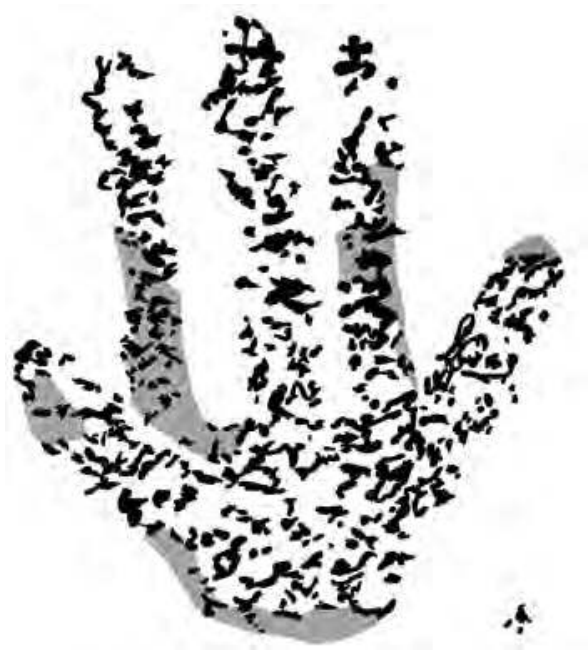

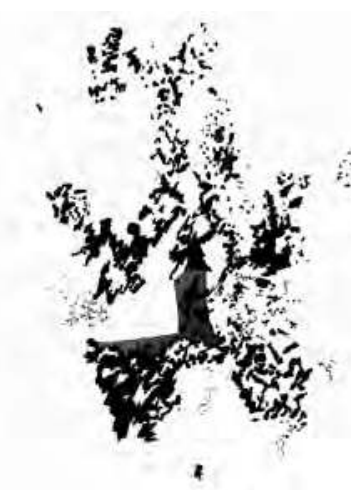
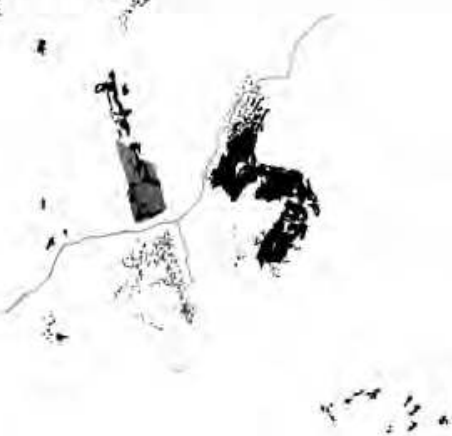

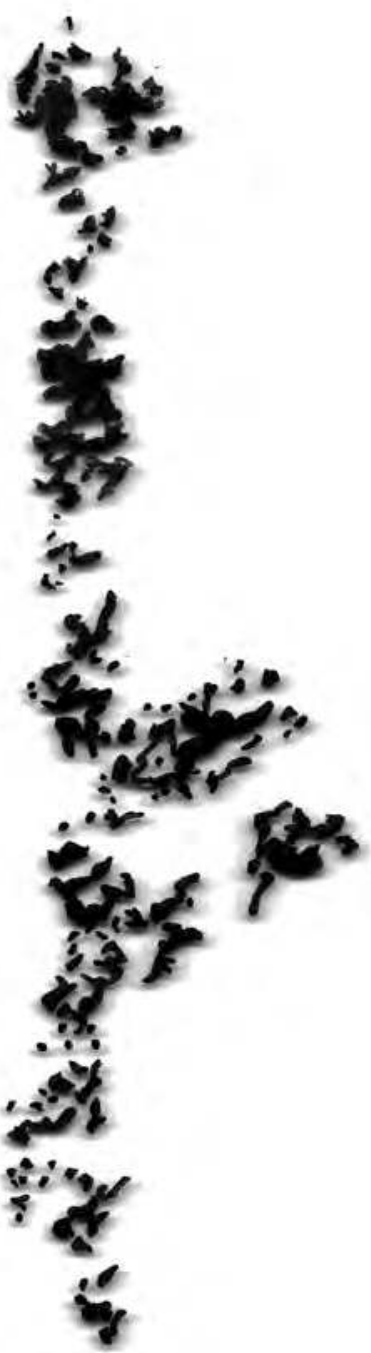

Fig. 2.4.11 : Examples of 1:1 copy of figures of Sarıaya-3 face A (A. Eyigör). 


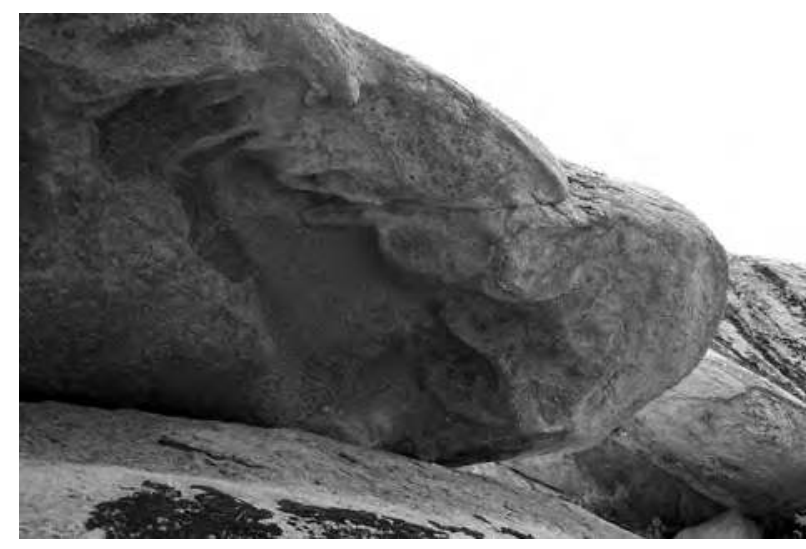

Fig. 2.4.12 : Sarıkaya-2 Face C (west)

(A. Eyigör).

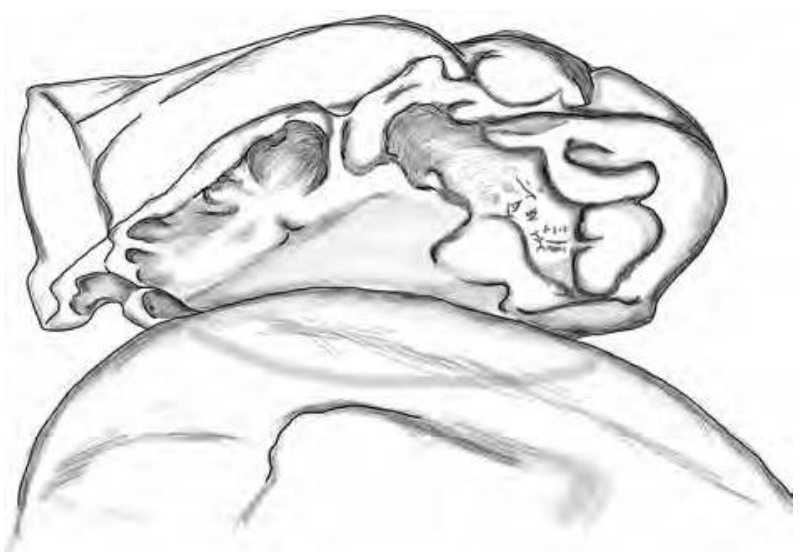

Fig. 2.4.14 : Sarıkaya-2, draft drawing of face $C$ (A. Eyigör).

located a few kilometers away on the other side of the valley (Fig. 2.4.16).

\section{A.2.3. Sarıaya-3}

There is a narrow paved road from west of Sarıkaya-2 leading down to other rock shelters with paintings near a stream (Fig. 2.4.17). The first one on this road, to the left, is Sarikaya 3 (Fig. 2.4.18). This boulder has two painted faces. Face A (west) shows four women figures and some traces of red pigment. Face B, on the other hand, is crowded with several couples, geometric patterns, single males and females, a hand and numerous dots in natural small cavities (Fig. 2.4.19 - Fig. 2.4.20). One man has a W shaped head like in Sarıkaya-1.

\section{A.2.4. Sarlkaya-4}

The same narrow paved road leading west from Sarıkaya-2 reaches down the stream, above which

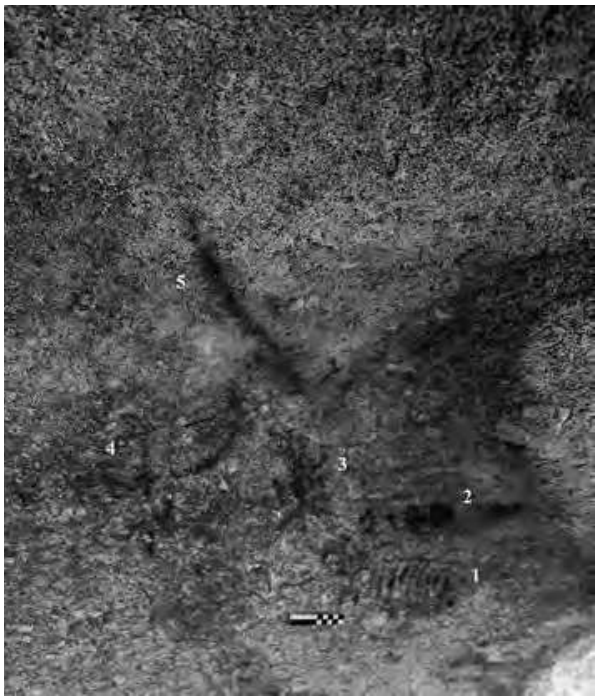

Fig. 2.4.13 : Enhanced image of Sarıaya-2 Face $\mathrm{C}$ (detail): 1. a goat/sheep in a fence (?) and a man hugged its neck can be seen;

2. a larvae/caterpillar in horizontal position; 3 . and 4. butterfly/moth; 5 . could be dragonfly (A. Eyigör).

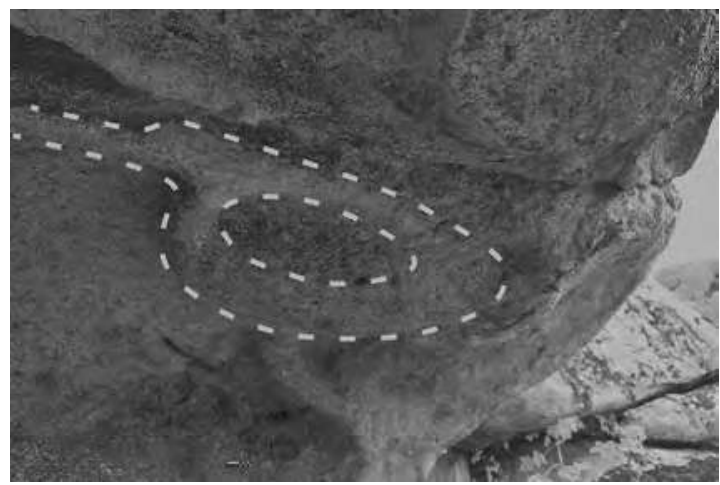

Fig. 2.4.15 : Some natural forms are highlighted with paint. Pigment traces are barely seen. On top right, a woman's silhouette was highlighted (A. Eyigör).

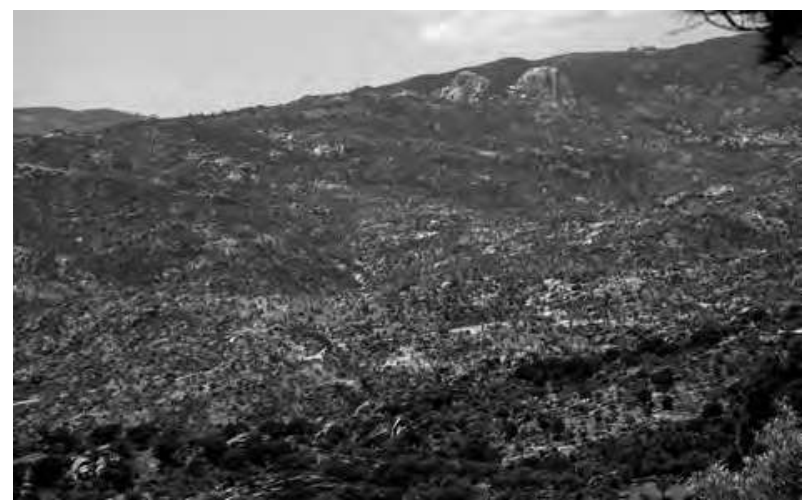

Fig. 2.4.16 : View of İkiztaş (twin rocks) from Sarıkaya-2 Face C (A. Eyigör). 


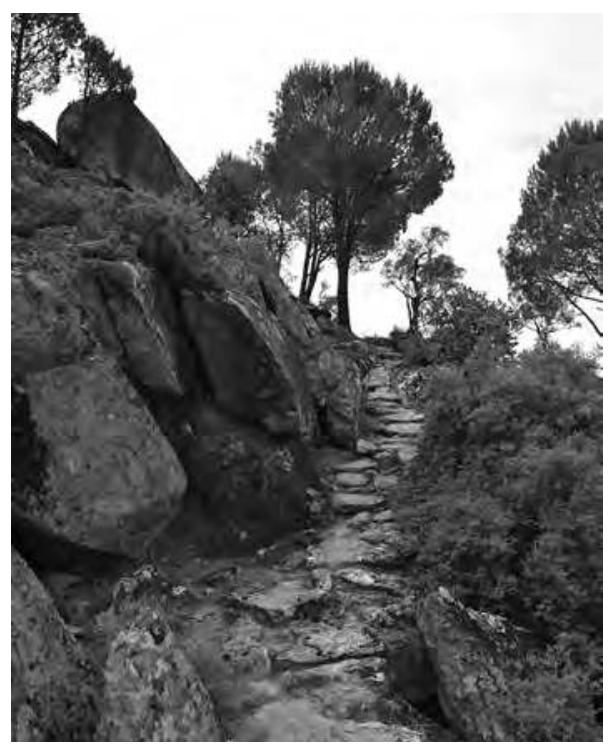

Fig. 2.4.17 : The path leading from Sarkaya-2 to Sarıkaya 3 (A. Eyigör).

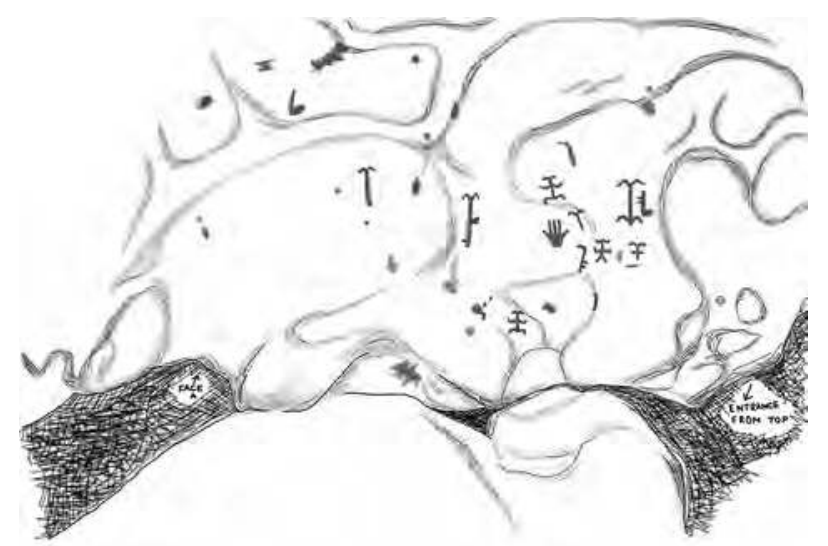

Fig. 2.4.19 : Draft drawing of Sarkaya-3 face B with positions of figures (A. Eyigör).

stands a large rock-shelter complex. Sarıkaya-4 (Fig. 2.4.21) is located close to this rock complex on the western edge of the stream. It has the shape of a small cave with low ceiling on grassy ground and there are numerous figures on its ceiling (Fig. 2.4.22). However, because of time limitation, the paintings could not be examined in detail.

\section{A.2.5. Sarıkaya-6}

Sarkkaya-6 is an individual rock laying directly on the earth and leaning against a large rock below Sarkkaya-3 (see Fig. 2.4.18 ; Fig. 2.4.23). Because of the rain water the figures are faded and covered with patina. Nonetheless, one seems to distinguish

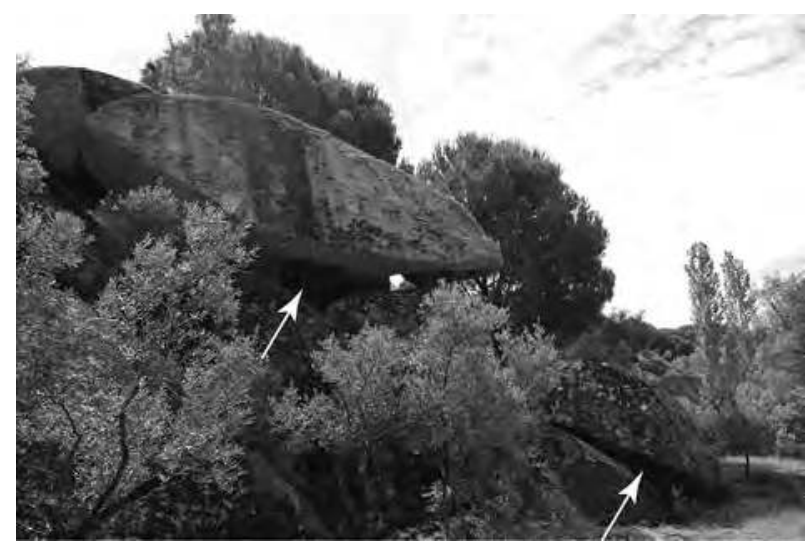

Fig. 2.4.18 : Sarıkaya-3 (left) and Sarıkaya-6 (right) view from west (A. Eyigör).

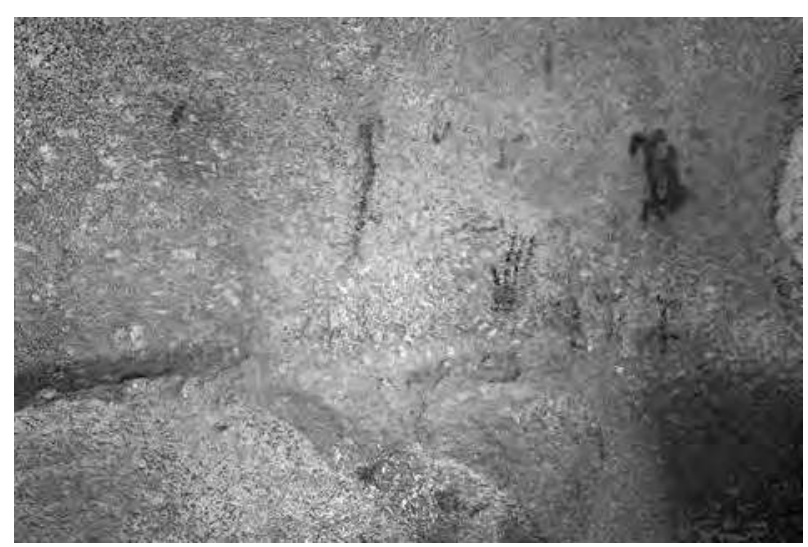

Fig. 2.4.20 : Enhanced images of Sarkaya-3 Face B (A. Eyigör).

several figures on its surface. A more detailed analysis of these paintings will be conducted in the coming seasons.

\section{A.2.6. Sarıkaya-7}

Sarıkaya-7 and Sarıkaya-11 form a corridor-like shape on the south side of the rock complex (Fig. 2.4.24). This is a gate to the courtyard in front and at the foot of Sarıkaya-3 and Sarıkaya-6. Sarıkaya-7 has two faces. Face A (northwest) lies directly above the stream bed (Fig. 2.4.25). This rock carries representations of human figures in elongated niches (Fig. 2.4.26). There are also numerous figures on face B. However, its surface is so detoriorated, be- 


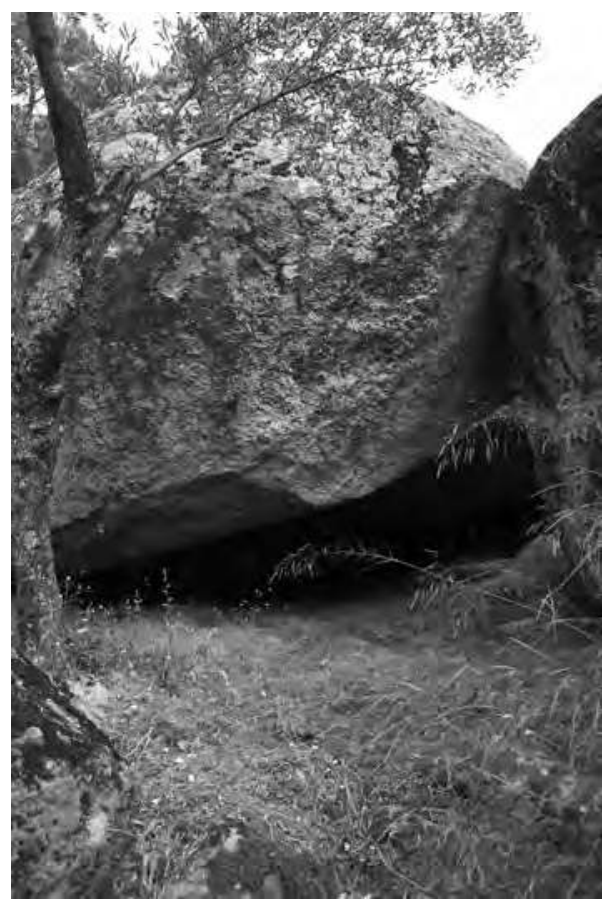

Fig. 2.4.21 : Sarıkaya-4 view from north (A. Eyigör).

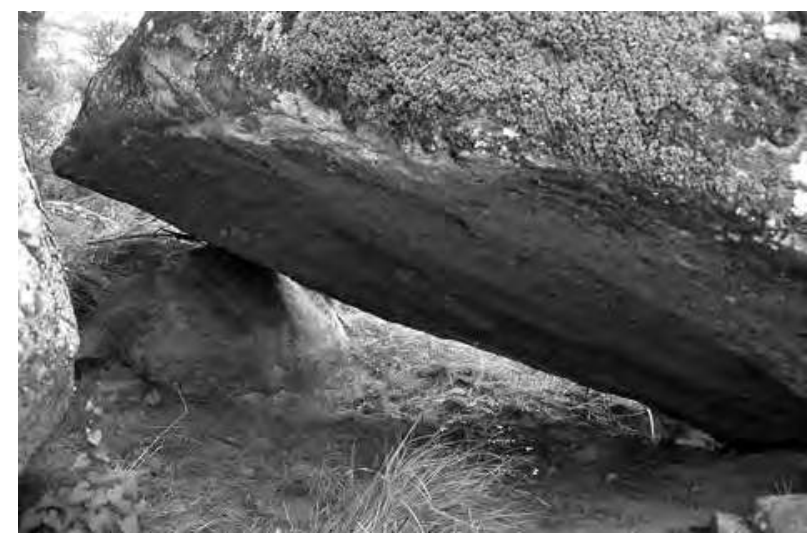

Fig. 2.4.23 : Sarıkaya 6 from the north (A. Eyigör).

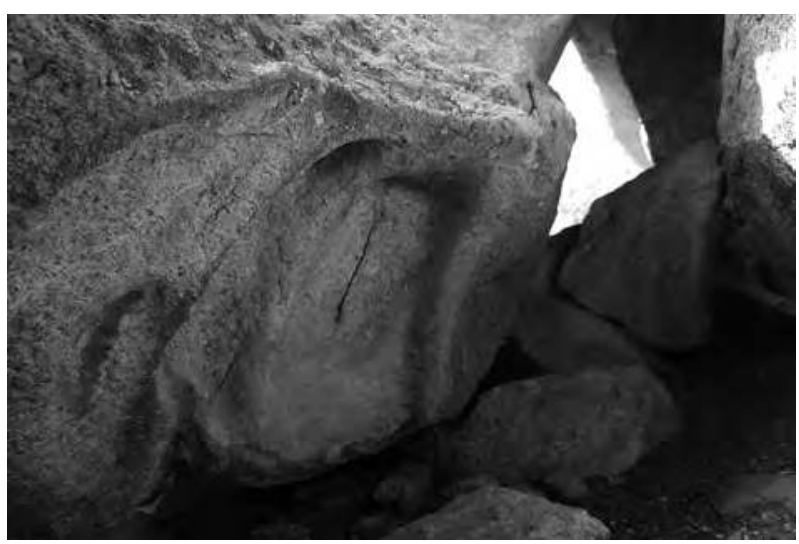

Fig. 2.4.25 : Sarıkaya-7 Face A (A. Eyigör).

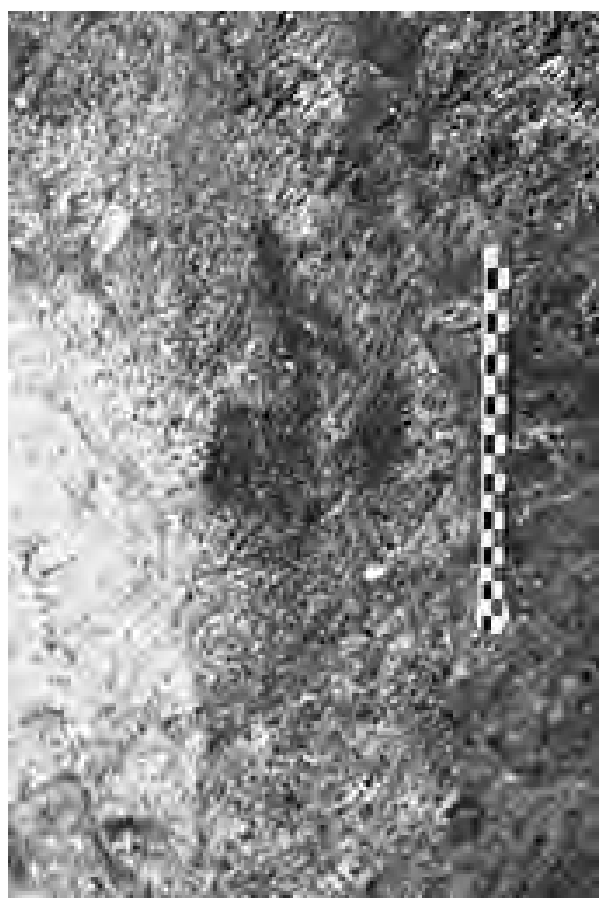

Fig. 2.4.22 : Enhanced image of detail from Sarıkaya-4 (A. Eyigör).

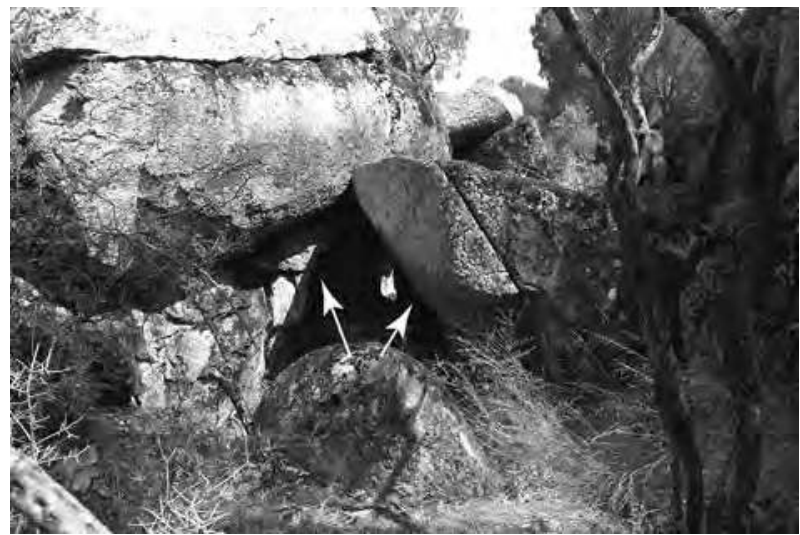

Fig. 2.4.24 : Sarkkaya-7 (right) and Sarkkaya-11 (left) from south (A. Eyigör).

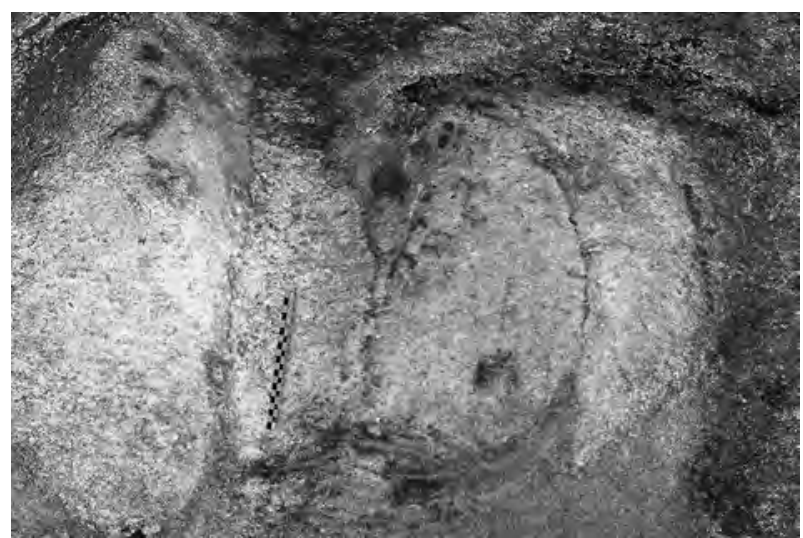

Fig. 2.4.26 : Enhanced image of Sarkaya-7 Face A detail (A. Eyigör) 
cause of direct exposure to sunlight and rain, that the figures can hardly be identified. A more detailed analysis of this rock-face will be done during the next seasons.

\section{A.2.7. Sarlkaya-8}

Sarkaya- 8 is a tall and wide rock block in the middle of the rock complex. Paintings are located on the upper and lower parts of one single face (east). In the lower part, in a long niche, a woman and some traces of two figures are seen on this surface. As for the upper part, it consists of a natural frieze, where figures have been painted. Because of the weathering of the rock, figures are very much faded and cannot be identified. At the same location we also found some anthropogenic erosions-like footprints on the lower rock. These could have been used to climb and paint/look at the upper paintings (Fig. 2.4.27).

\section{A.2.8. Sarıaya-9}

Sarıkaya-9 is located very close to the northeast of the previous rock-complex. It is a narrow shelter (Fig. 2.4.28). It now serves as an animal shelter. Two woman figures and a zigzag have been spotted in the natural niches of its ceiling (Fig. 2.4.29).

\section{A.2.9. Sarlkaya-10}

Sarikaya-10 is located right in the middle of the rock-complex, below Sarkkaya-8. It has two faces: A (north) and B (south). On the face A, there is no painting, but two holes have been clearly carved on the top of the rock (Fig. 2.4.30). This arrangement is looking directly at the woman figure of Sarıkaya-8 (lower part). The face B carries several representations of human figures and hand prints. They are much faded because of the rain water (Fig. 2.4.31).

\section{A.2.10. Sarıkaya-11}

Sarıkaya-11 is a wide boulder, located inside the rock-complex. It has two faces. Face A (northeast) is located inside the bed stream, while the face B (southeast) is on the south side of the rock-complex, opposite to Sarıkaya-7. Some marks left in the rock do not seem to be natural. It shows at least 7 vertical incisions, 5 horizontal thick lines and 4 cupules (Fig. 2.4.32). The face of the rock has greatly suffered from weathering and there is a large lacuna on the right bottom part of the surface, probably caused by the water flow of the stream when heavy rains fall in the region. It is therefore possible that the incisions were more numerous in the original state of the occupation. The dating of these cuttings is not possible yet, however we hope to find some evidences to define the tool used for making these incisions by microscopic examination in the near future.

On face B, one can distinguish human figures, highlighted areas and pigment traces. There is a group of three persons in the center of the composition (Fig. 2.4.33).

\section{A.2.11. Sarıaya-12}

Sarıkaya-12 is also located in the stream bed, north of Sarıkaya-7 and opposite of Sarıkaya-11 face B. Human figures and patterns can bee seen, although they all are very much faded (Fig. 2.4.34).

\section{A.2.12. Sarıaya-13}

Sarıkaya-13 was found on the last day of the survey. It is dominating the rock-complex, above Sarkkaya-8. It is particularly complicated to access this rock (and requires many detours), although once there the location is fairly comfortable for seating (Fig. 2.4.35).

This is probably the most impressive collection of paintings discovered so far at Sarkaya, both in term of the size of the figures (some of them are $30 \mathrm{~cm}$ high) and of their variety and particularities. The rock face carrying the paintings is slightly leaning forward and does not really provide a shelter. This explain why most of the paintings are particularly faded, due to the direct sunlight and running rainwater. Some photos were taken, but detailed examination could not be performed in 2017 .

Our rapid evaluation could however distinguish at least three different crowded compositions. Among the many unique peculiarities of these paintings one notices: a woman with a triangular shaped head/hat (Fig. 2.4.36); two large women associated with nine large dots/vertical lines (Fig. 2.4.37); dots above some individuals head; and finally and puzzling representation of a lonely zoomorphic man. His arms are raised up and prolonged with curved line turning downward and giving a wing-effect to the overall shapes (Fig. 2.4.38).

The location of the rock (on top and hardly accessible) and the characteristics of the figures are emphasizing the exclusivity of the area: this rock could have been used for special events. 


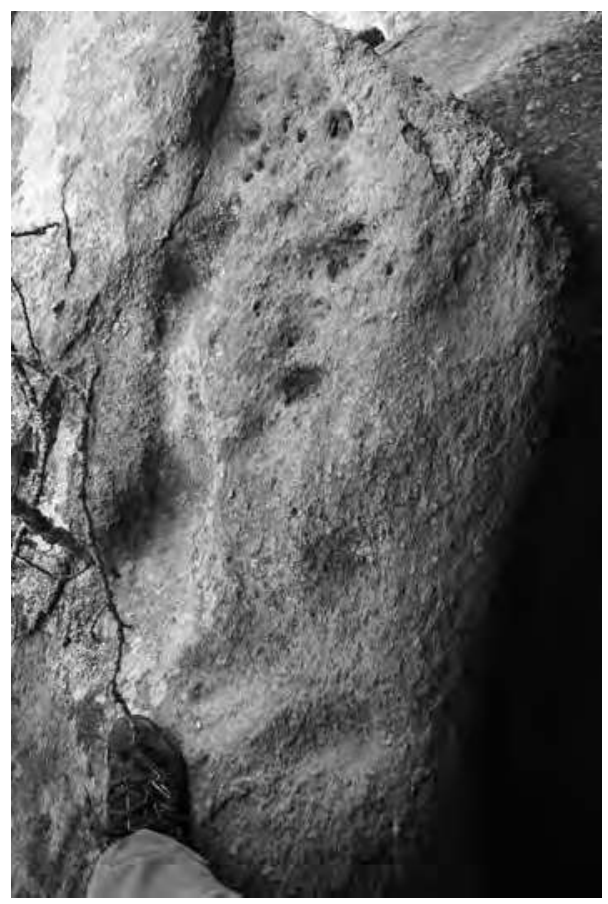

Fig. 2.4.27 : Some anthropogenic erosions like footprints on the lower rock of Sarıaya-8 (A. Eyigör).

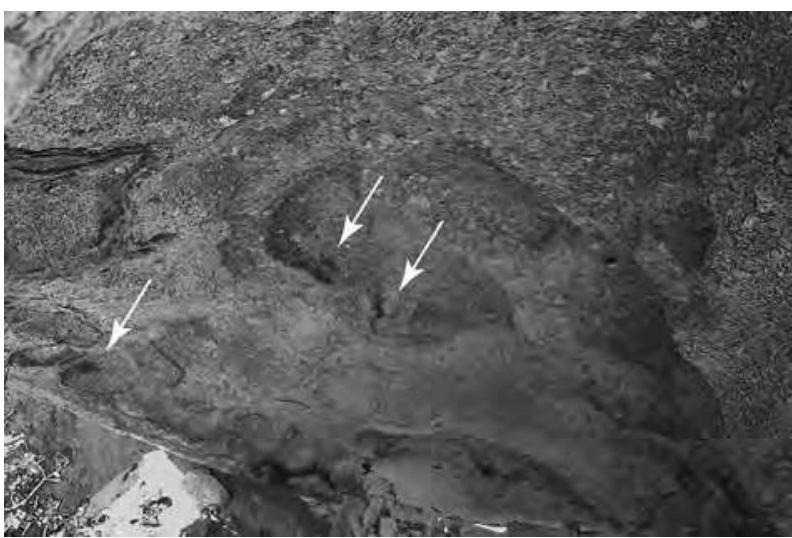

Fig. 2.4.29 : Enhaced image of Sarıkaya-9 (A. Eyigör).

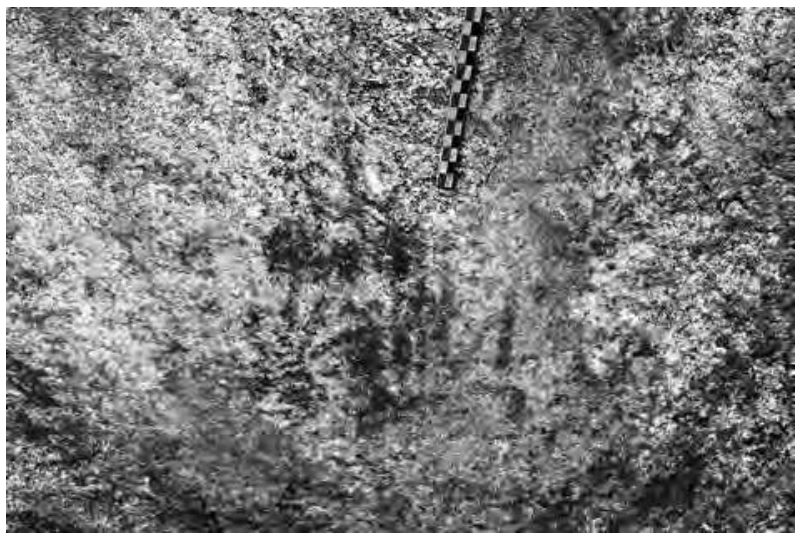

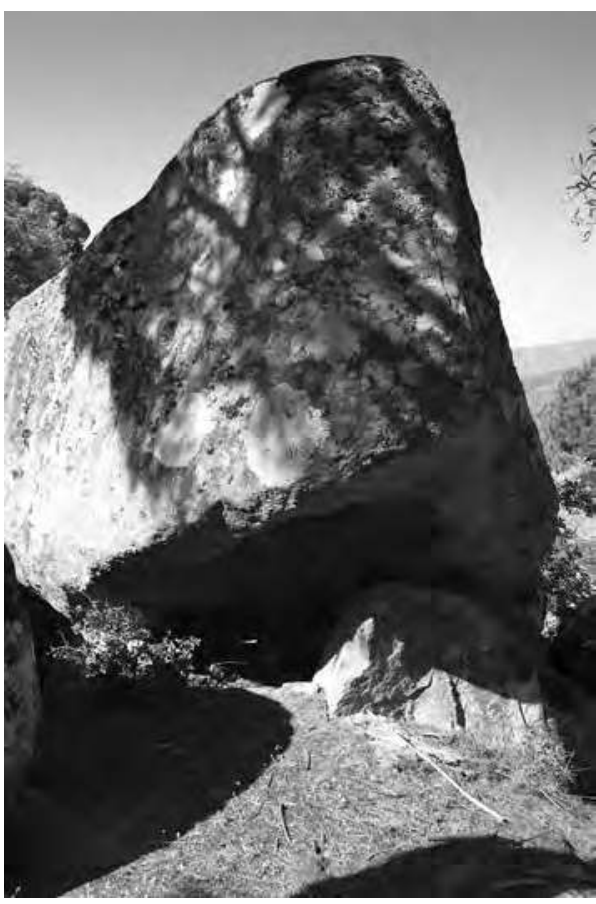

Fig. 2.4.28 : Sarıkaya-9 from the North (A. Eyigör).

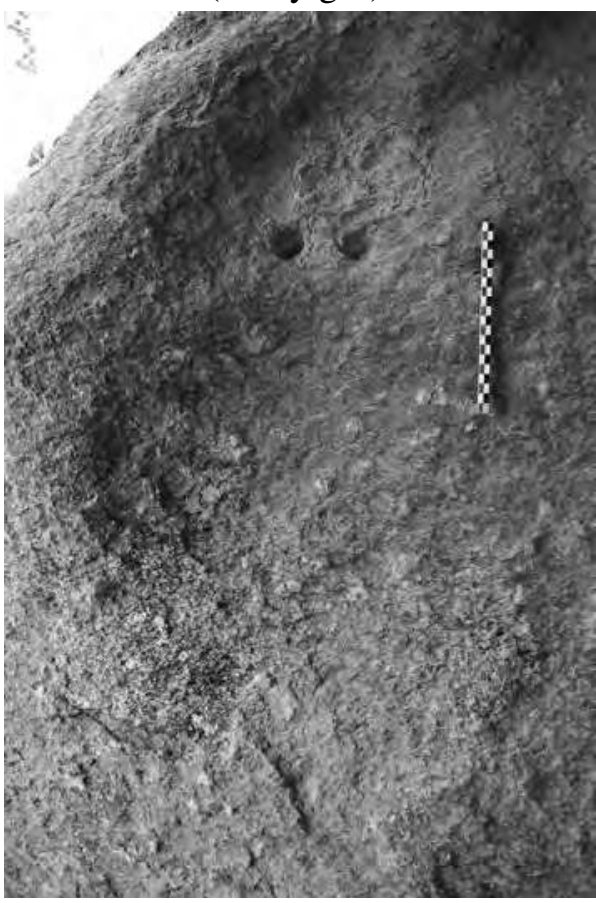

Fig. 2.4.30 : Sarıkaya-10 face A (north) (A. Eyigör).

Fig. 2.4.31 : Enhanced image of Sarıkaya-10 face B (south) detail (A. Eyigör). 


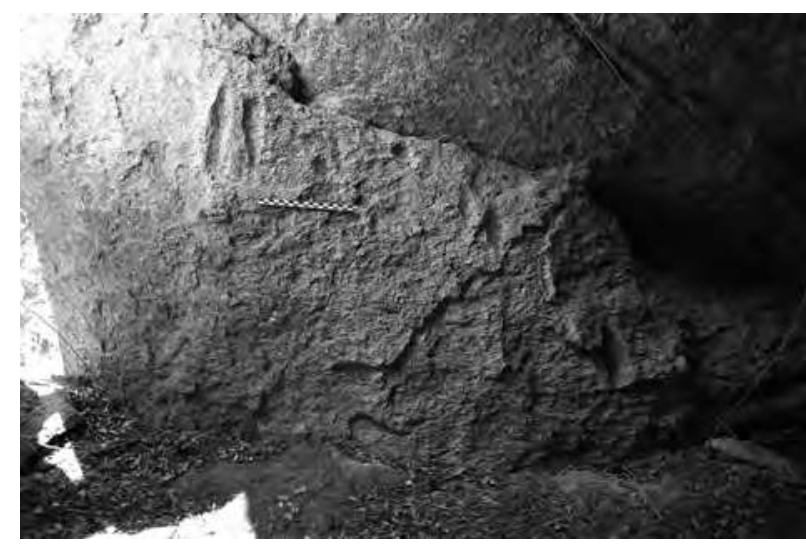

Fig. 2.4.32 : Sarkaya-11 face A, vertical and horizontal lines and some cupule can be seen on surface (A. Eyigör).

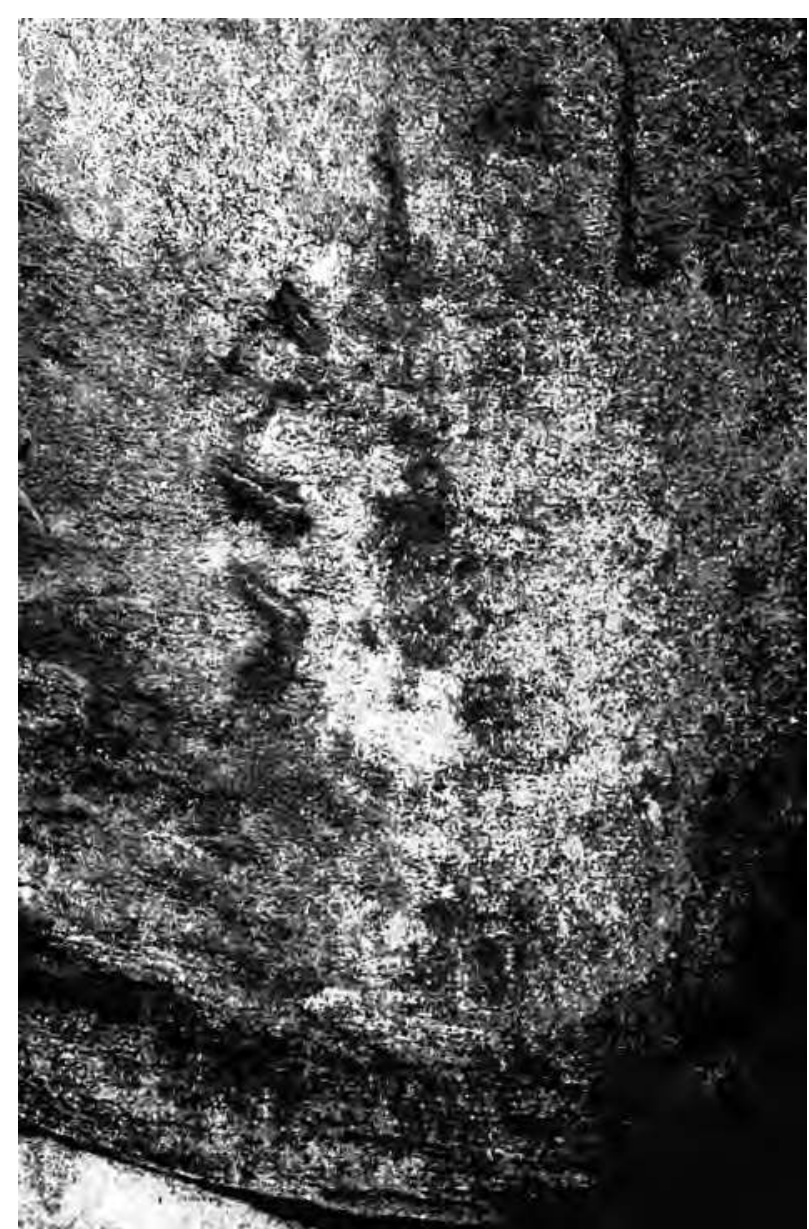

Fig. 2.4.34 : Enhanced image of Sarkaya-12 (A. Eyigör).

Fig. 2.4.37 : Enhanced image of Sarıaya-13 part $L$

(A. Eyigör).

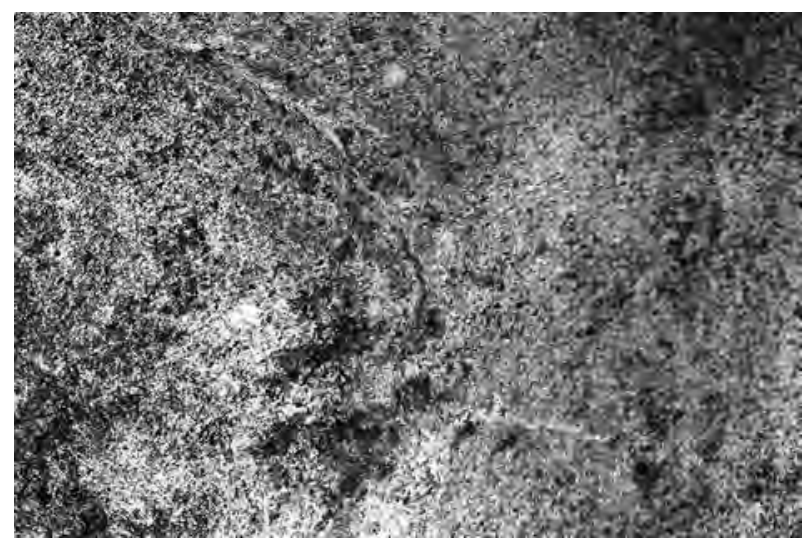

Fig. 2.4.33 : Enhanced image of Sarıkaya-11 face B detail (A. Eyigör).

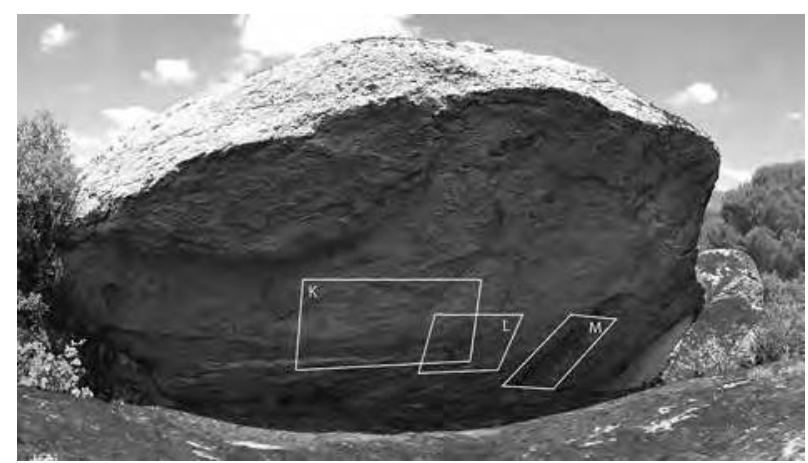

Fig. 2.4.35 : Panaromic view of Sarıkaya-13 (A. Eyigör).

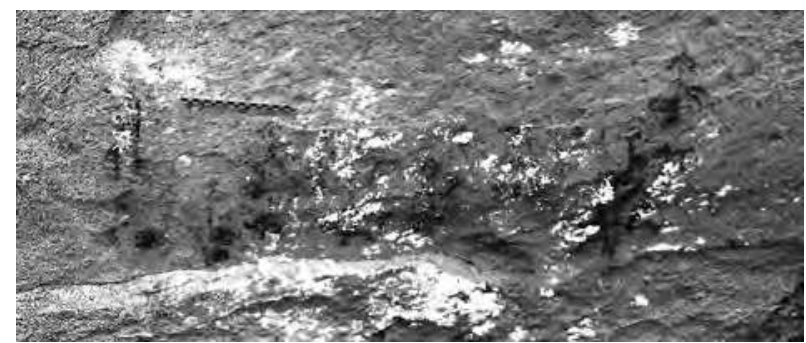

Fig. 2.4.36 : Enhanced image of Sarıkaya-13 part K (A. Eyigör).

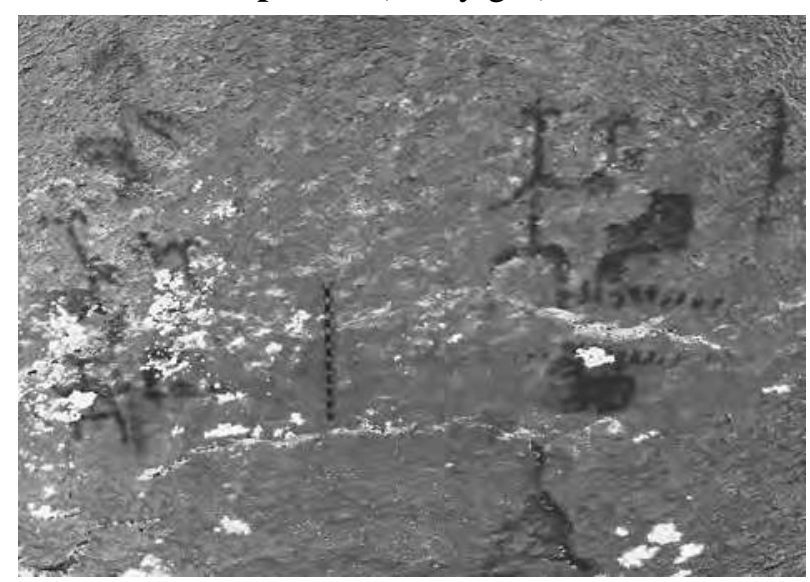




\section{A.2.13. Sarlkaya-14}

Sarıkaya-14 is located along the pathway between Sarıkya-2 and Sarıkaya-3 on the eastern side. Here, three rocks form some kind of a natural gateway (Fig. 2.4.39) Pigments of red paint that might represent a woman and a zig-zag have been spotted. Further investigations will take place during the 2018 season.

\section{A.2.14. Sarlkaya-15}

Sarıkaya-15 is an elongated piece of rock that leans close to Sarıkaya-1. Its painting are hardly seen since as they are located in a niche looking down and partly hidden by the summit of the boulder against which it leans (Fig. 2.4.40). There are some human figures represented. Further investigations will take place during the 2018 season.

\section{A.2.15. Sarlkaya-16, 17 and 18}

Some pigment traces in three more rock shelters were found close to the stream down toward the eastern side of the rock-complex. They will be examined in the next season.

\section{A.3. Conclusion}

Although it is much too early to start drawing conclusions to the discovery of this series of rock-paintings, many questions already come to mind. Among the most urgent are:

- Would the differences noticed between the paintings in North Latmos and the paintings in Sarıkaya indicate the existence of different living groups/clans in the region?

- Was this area a meeting point of different groups? If there were different groups, what was their relationships? Were these paintings showing or regulating the relationships among them?

- What was the aim of making these paintings? Were they ordinary or special? What kind of criteria was considered for choosing the locations of paintings?

- Was this area a stop for the community or were they living here close? Were there any stationary or temporary living areas (caves for example) around?

- When was the road paved? Was it related to the rock paintings?

This survey will continue for at least 4 seasons. During our studies, we will try to find some an- swers to these questions and we hope to find new rock-paintings and prehistoric materials to better date and understand the life in prehistoric time in Latmos.

\section{B. Dikmen Tepe - Habitat/Ferme (par O. Henry)}

A environ $250 \mathrm{~m}$ plus au Nord de la zone qui comprend les peintures rupestres, à une altitude de $424 \mathrm{~m}$, nous avons trouvé une modeste zone d'occupation s'étendant entre deux éminences rocheuses, installée à cheval sur un col étroit entre deux vallées. Il s'agissait probablement d'un complexe agricole, à en juger par les nombreux vestiges d'éléments de production. Le site est bien desservi avec des sources d'eau naturelles, dont deux furent aménagées en fontaines. L'une au Nord-Est (SAR005A) domine une série de terrasses étroites et hautes (SAR005B) (Fig. 2.4.41). Les murs de soutènement de ces terrasses sont faits de petites moellons. Ils sont élevés et présentent un important fruit qui semble avoir servi de contrefort. A l'Ouest, sous un pic rocheux, on trouve une pierre de presse oblongue, probablement destinée à la production d'huile d'olive (SAR005) (Fig. 2.4.42). Il est possible que cette pierre ne se trouve pas dans sa position d'origine car aucun autre élément lié à cette activité (contrepoids, cavité de prelum, etc.) n'a été observé dans les environs immédiats. D'autre part, à quelques mètres au Sud et au-dessus de ladite pierre se trouve un autre petit complexe. Il comprend un grand rocher affleurant, dont le dessus a été taillé afin d'offrir une courte plate-forme. Au milieu de cette plate-forme, un trou de mortaise/poteau circulaire a été aménagé. A environ $1,5 \mathrm{~m}$ au Sud, on remarque que le rocher environnant a aussi été taillé, à angle droit, et il est très possible que ces deux éléments appartiennent à la même structure (Fig. 2.4.43).

Au Sud-Est, on remarque l'aménagement de nombreuses terrasses, entourant le site et le sommet rocheux, au Sud. Certains murs, construits avec des blocs hétérogènes, et dont les plans en $\mathrm{Pi}$ ou en $\mathrm{L}$ ne semblent pas correspondre à du terrassement, semblent devoir être identifiés à autant d'habitats. Ils pourraient être des vestiges de petites maisons. Heureusement (et contrairement à la plupart des autres sites découverts cette année), nous avons pu collecter beaucoup de poterie sur place. L'étude préliminaire de ce matériel donne une large fourchette d'occupation, de la période hellénistique jusqu'à la période byzantine (Fig. 2.4.44). Il faut aussi mentionner la découverte d'une hache polie brisée, trouvée dans la pente sous le site, au Nord 


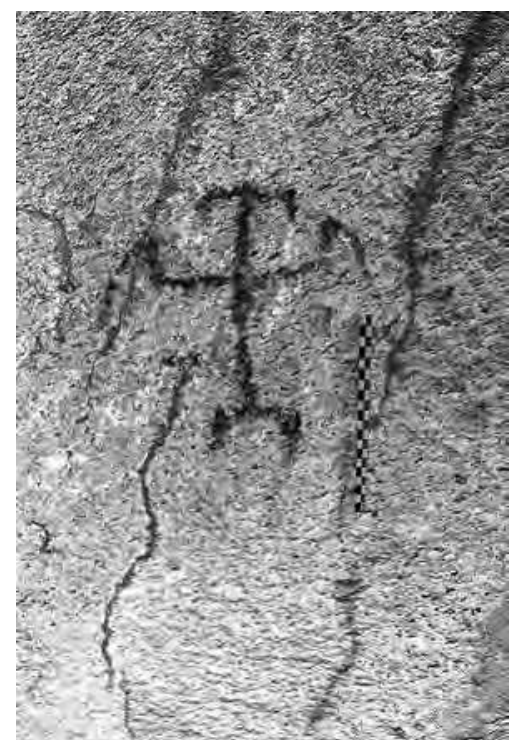

Fig. 2.4.38 : Enhanced image of part M (A. Eyigör).

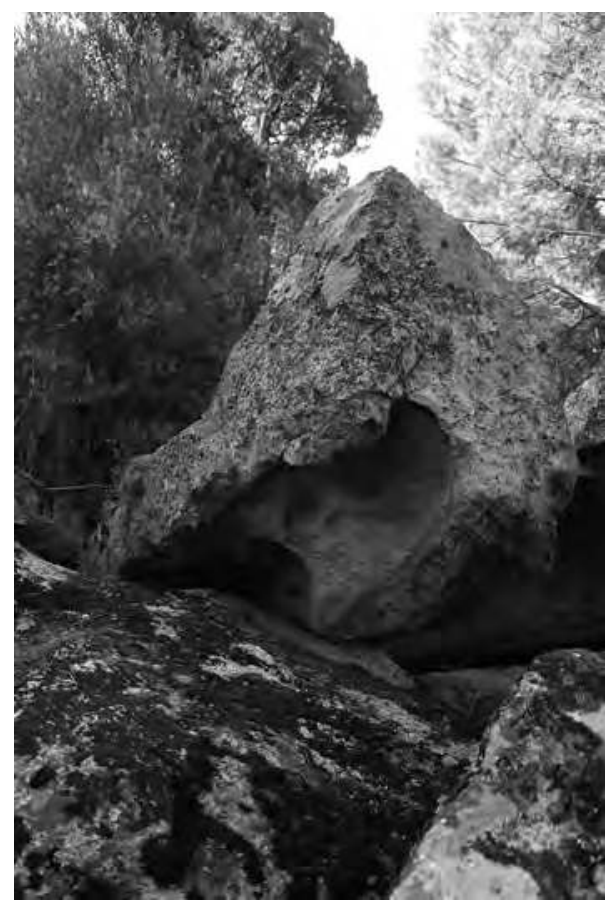

Fig. 2.4.40 : Sarıkaya-15 view from northwest (A. Eyigör).

(Fig. 2.4.45). Il est impossible d'affirmer qu'il s'agit d'un indice d'une occupation plus ancienne de la zone, au cours de laquelle les formations rocheuses aurait pu servir d'abri, ou si cette hache polie fut collectée dans la zone, relativement proche, des peintures rupestres et déplacée ici dans l'antiquité. L'absence totale de vestiges funéraires (antiques) est surprenante, compte tenu de la longue période d'occupation indiquée par la poterie. Notons cepen-

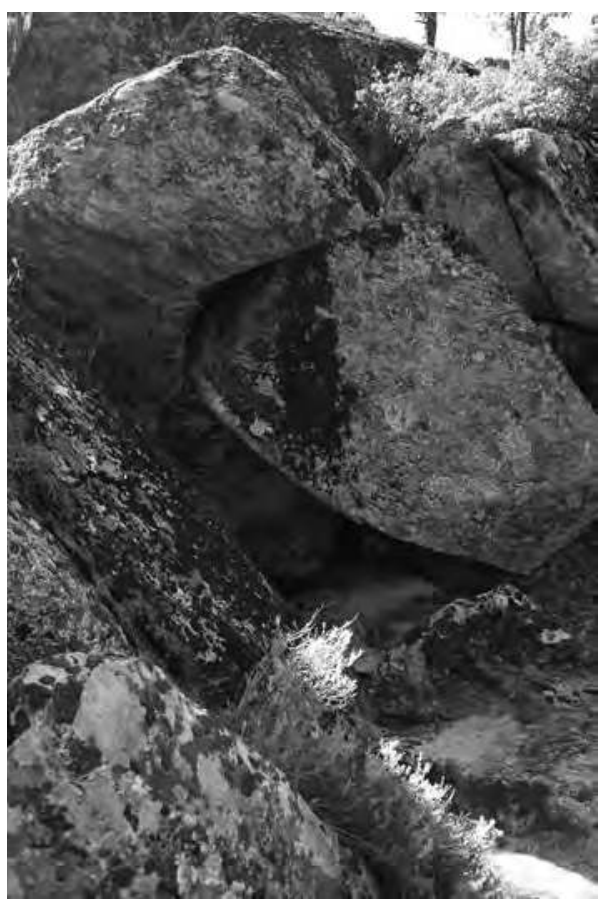

Fig. 2.4.39 : Sarıkaya-14 seen from the pathway (West) (A. Eyigör).

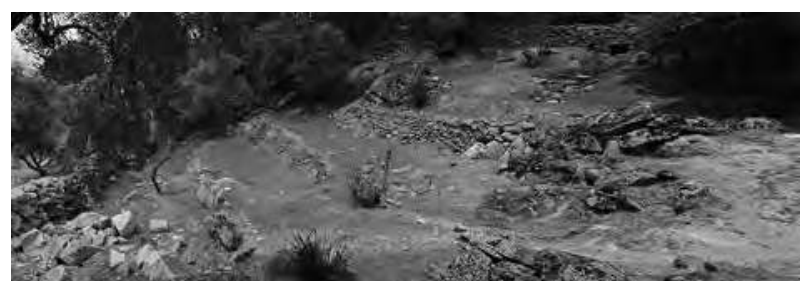

Fig. 2.4.41 : Les terrasses dominées par une fontaine aménagée à Dikmen Tepe (O. Henry).

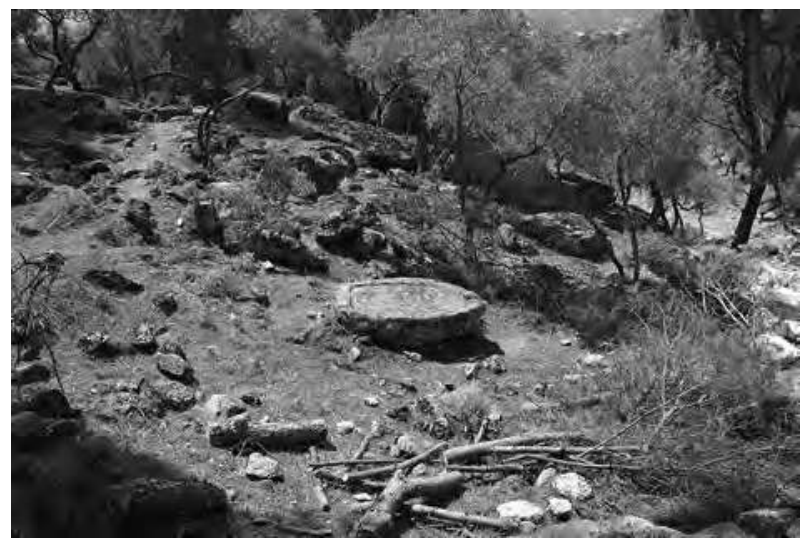

Fig. 2.4.42 : Pierre de maie à Dikmen Tepe (O. Henry).

dant la présence d'un cimetière musulman relativement ancien, au pied de la pente, à une centaine de mètres au nord. La pérennité de l'occupation de ce site, sur plusieurs siècles, pourrait s'expliquer à la fois par la présence de nombreuses résur- 

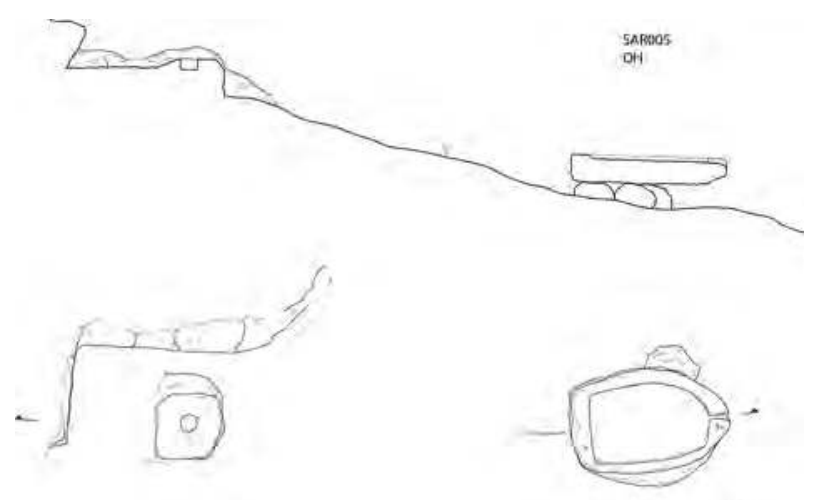

(4)

$\therefore-$

Fig. 2.4.43 : L'aménagement de presse à Dikmen Tepe (O. Henry).
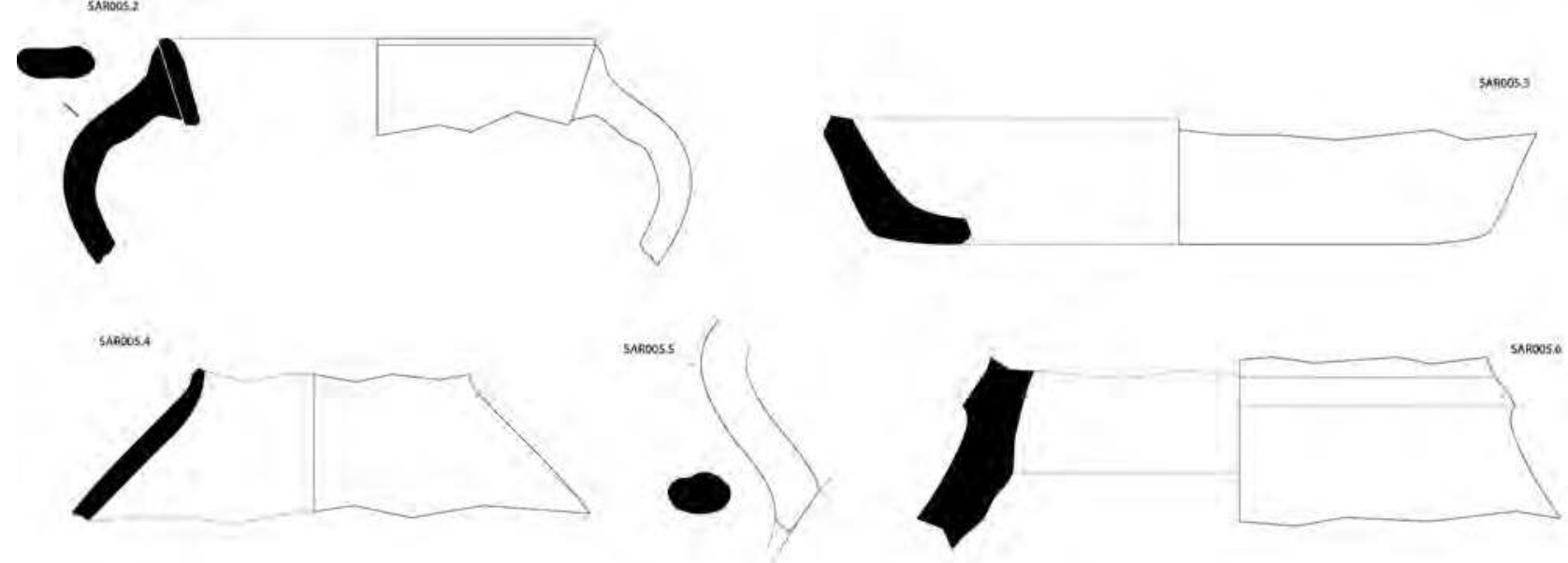

SAROOSOS
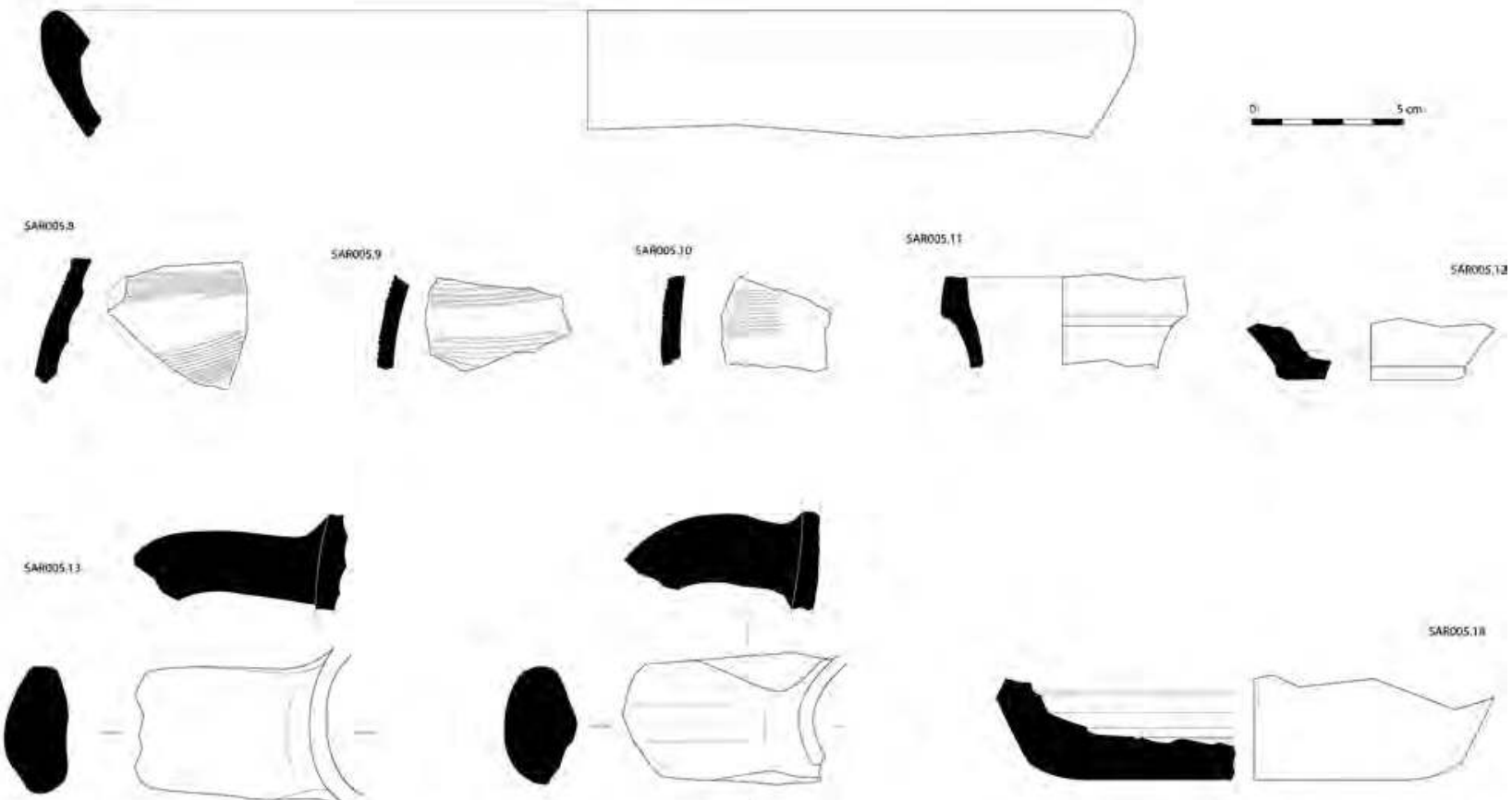

Fig. 2.4.44 : Exemple de tessons de céramique récoltés à Dikmen Tepe (O. Henry). 


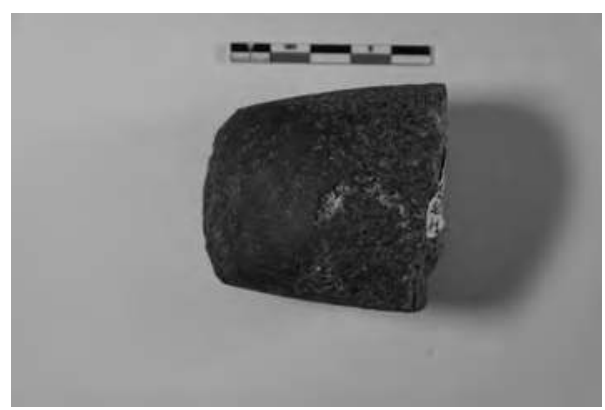

Fig. 2.4.45 : Hâche polie récoltée à Dikmen Tepe (O. Henry).

gences d'eau clair et par la localisation particulièrement stratégique du site, au point de contrôle d'un des principaux passages entre la plaine alluviale de Mylasa et les hauts plateaux du Latmos.

\section{Asarlık Tepe - Fortification (par O. Henry)}

A plus de $1,5 \mathrm{~km}$ au Nord-Est du village de Sarıkaya se dresse une colline rocheuse appelée Asarlık tepe (la colline du château), dont l'altitude atteint $743 \mathrm{~m}$. L'enquête menée au sommet de cette colline a révélé une grande quantité d'informations (Fig. 2.4.46). Il s'agit d'une zone oblongue, plus ou moins plate, de $200 \mathrm{~m}$ de long, dans la direction Nord-Est/Sud-Ouest, et environ $80 \mathrm{~m}$ dans la direction Est/Ouest. Cet espace est encadré à l'Est et au Sud-Ouest par des falaises abruptes, tandis que ses deux extrémités, au Nord et au Sud, sont marquées par deux pics naturels (respectivement points $\mathrm{C}$ et $\mathrm{E}$ de la figure 2.4.46). Sa pente ouest est assez douce. La topographie et l'emplacement d'Asarlık tepe lui donnent une position dominante sur une grande zone comprenant les villages de Ketendere à l'Ouest, Sarıkaya au Sud et la vallée profonde de Kara Deresi à l'Est. Cela explique probablement pourquoi le sommet de la colline a été choisi pour être fortifié dans l'antiquité. Le système de fortification ne concernait que les zones où la colline n'était pas naturellement défendue par les formations rocheuses.

A l'Ouest, là où la topographie est la plus douce, se dresse en effet un mur impressionnant érigé entre deux grands rochers (points A et B) (Fig. 2.4.47), fermant le seul accès au sommet sur cette partie de la colline. Le mur mesure près de $50 \mathrm{~m}$ de long et $2 \mathrm{~m}$ de large. Il est rectiligne et forme un redent à mi-chemin. Il est extrêmement bien conservé et semble avoir été utilisé à la fois comme mur défensif et mur de soutènement, compte tenu de la différence des niveaux de sol à l'intérieur et à l'extérieur du périmètre. Sa hauteur maximale sur la face ex-

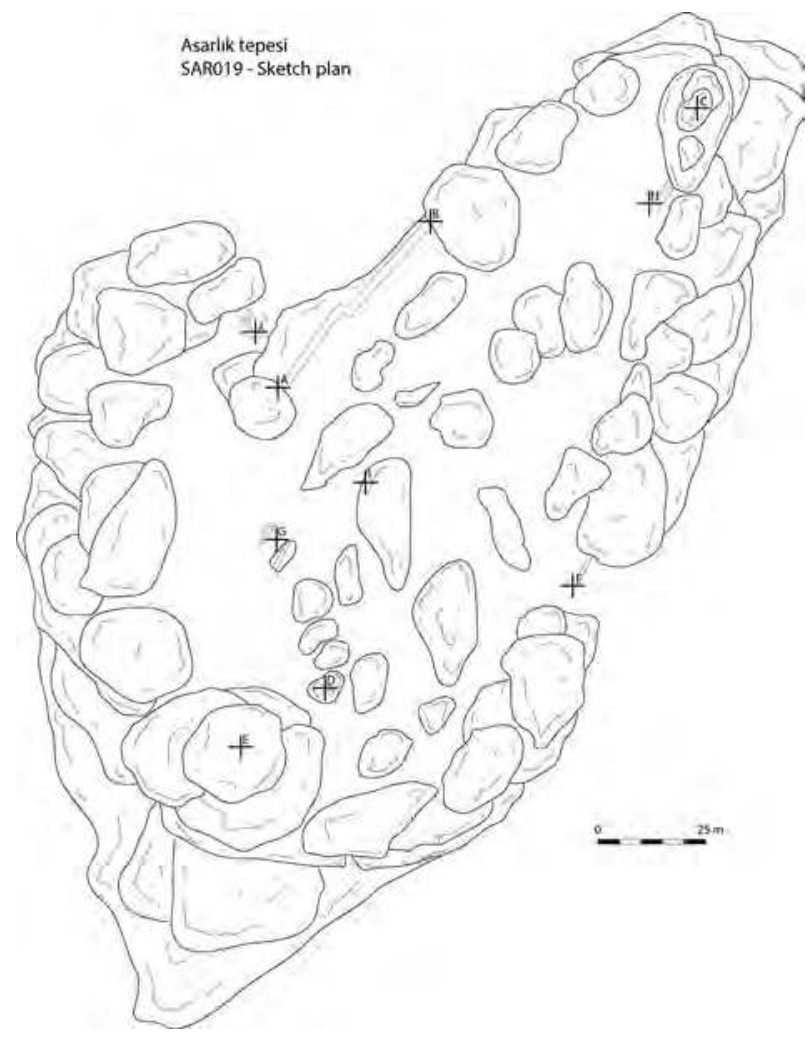

Fig. 2.4.46 : Croquis des vestiges de Asarlık Tepe (O. Henry).

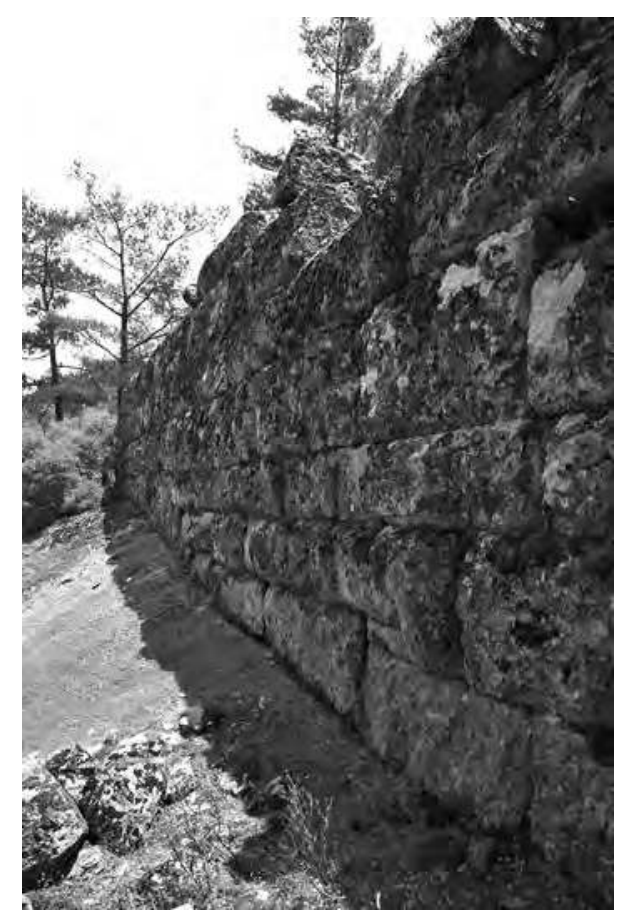

Fig. 2.4.47 : Vue de la section nord du mur de fortification de Asarlık Tepe (depuis le Sud-Ouest) (O. Henry). 
terne atteint jusqu'à 4,60 m, avec jusqu'à sept assises composées de pierres de taille, tandis que son parement interne, constitué de blocs plus petits et hétérogènes, n'est visible que sur $1,35 \mathrm{~m}$ au maximum. Les fondations de la face externe sont assises directement sur le socle rocheux aménagé en escalier. Les techniques de construction du mur, offrant des parpaings arrangés en carreau et boutisse, avec bossage et ciselure d'angle (Fig. 2.4.48), sont typiques de la période hellénistique. Les blocs utilisés dans la construction sont particulièrement massifs, avec des assises pseudo-isodomes variant entre 44 et $68 \mathrm{~cm}$ de hauteur, tandis que certains parpaings atteignent jusqu'à 2,70 m de longueur (Fig. 2.4.49). Les boutisses, traversent l'intégralité du mur et ont une longueur de 2,08 m (Fig. 2.4.50). Sur la section nord, on remarque la présence d'une chantepleure, aménagée dans la $6^{\mathrm{e}}$ assise, depuis l'arase du mur (Fig. 2.4.51).

Le côté oriental de la colline est presque entièrement naturellement défendu avec de hautes falaises, seulement traversé par une petite vallée peu profonde. L'accès vers et depuis cette vallée a également été fermé par un mur (point F). Ce mur, très mal conservé, est nettement moins bien construit que le long mur occidental. Il a été érigé à l'aide d'un matériel hétéroclite et son état de conservation actuel lui donne d'avantage l'aspect d'un mur de soutènement plutôt que d'une structure défensive.

De nombreux blocs équarris de grandes dimensions (point $\mathrm{J}$ ) ont été repérés sous le long mur occidental vers son extrémité sud. Ces blocs n'appartiennent pas au mur qui semble intact à cet endroit. Par contre les nombreuses marques portés par les blocs, et leur emplacement dans un couloir naturel étroit entre deux formations rocheuses qui longent le mur (Fig. 2.4.52), semblent indiquer qu'ils formaient autrefois probablement une porte d'accès.

Bien qu'il soit très possible que l'endroit n'ait jamais été utilisé comme habitat, on note, à l'intérieur du circuit, de nombreuses traces d'occupation. En effet, l'espace circonscrit par les fortifications, naturelles et construites, ne propose que très peu d'endroits dégagés entre les nombreux rochers disséminés. Parmi les vestiges d'occupation, on remarque : des traces de tailles longitudinales dans le rocher sous le sommet du sommet $\mathrm{du}$ sud (point $\mathrm{C}$ ), probablement les marques de fondation d'un mur/tour ; un groupe de cavités arrondies (bothroi ?) sur le dessus d'un rocher qui aurait pu être connecté à des libations (point D) ; des traces d'extraction et quelques blocs dispersés (point $\mathrm{G}$ ) ; un possible escalier qui mène au pic rocheux septentrional (point $\mathrm{H}$ ) ; des mortaises de charpente au-dessus d'un abri sous roche naturel (point I) (Fig. 2.4.53).

\subsubsection{KOCALAN - HABITAT (par O. Henry)}

Le site est localisé sur une terrasse plate naturelle sur le Kocalan Sr. La zone est orientée NordEst/Sud-Ouest, entre 600 et $650 \mathrm{~m}$ d'altitude et est encadrée par deux vallées. Les vestiges anciens, déjà connus par les autorités locales, bien que jamais documentés, sont nombreux et éparpillés sur une vaste zone. Ils comprennent plusieurs tombes rupestres, de nombreuses terrasses construites, une citerne, des traces de production d'huile et de vin et des vestiges de grands bâtiments.

\section{A. Les tombes (KOCA001, KOCA002, KOCA003 et KOCA010)}

Quatretombesontétérepérées.Elles sontchacune isolée et localisées dans la partie basse du site. Trois d'entre-elles appartiennent au type des sarcophages rupestres (KOCA001, KOCA002, KOCA010) (Fig. 2.4.54-2.4.55). Elles sont typiquement aménagées au sommet d'un rocher affleurant dont la partie haute est taillée de manière à offrir une plateforme. La fosse rectangulaire est creusée au centre de cette plate-forme et souvent entourée de canaux peu profonds qui visent à drainer l'eau de pluie. Les parois internes de la fosse sont marquées d'un ressaut sur chacun des quatre côtés, divisant la cuve en une partie supérieure et une partie inférieure. Les tombes disposent chacune d'un imposant couvercle à bâtière monolithique, orné sur les côtés de reliefs de préhension. La quatrième tombe (KOCA003) est une fosse rectangulaire simple, creusée dans le sol rocheux au sein d'une grande terrasse naturelle. Elle a fait l'objet de fouilles illégales. Contrairement aux sarcophages rupestres qui sont souvent isolés, ce type de tombes appartient souvent à de grandes nécropoles à forte densité d'individus. Il est donc probable que l'intégralité de la terrasse qui porte la tombe KOCA003 contienne davantage de sépultures.

En général, l'enquête n'a révélé aucun matériel archéologique qui nous aurait permis de déterminer une fourchette chronologique pour les tombeaux. Néanmoins, des parallèles typologiques sont bien connus dans la région, en particulier dans la nécropole de Labraunda. Il semble donc que les tombes 001,002 et 010 découvertes à Kocalan puissent être datées à l'époque hellénistique précoce, et qu'elles furent probablement réutili- 


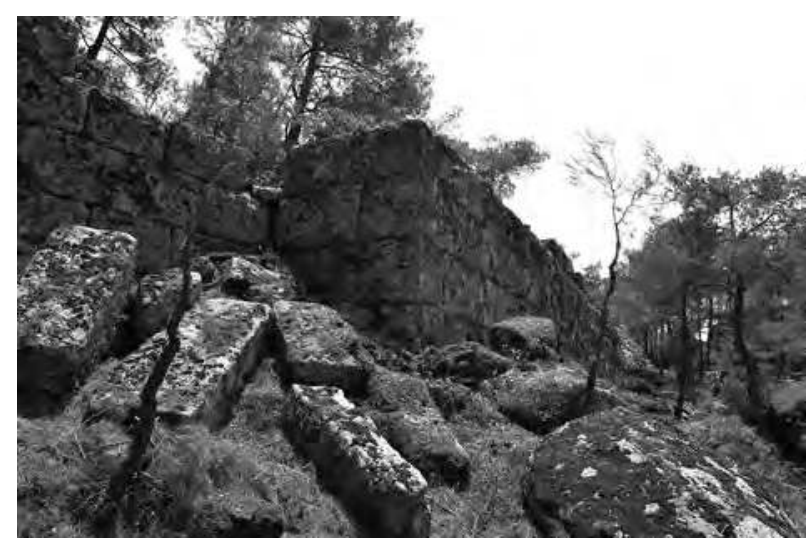

Fig. 2.4.48 : Vue du décrochement du mur de fortification de Asarlık Tepe (depuis le Nord) (O. Henry).

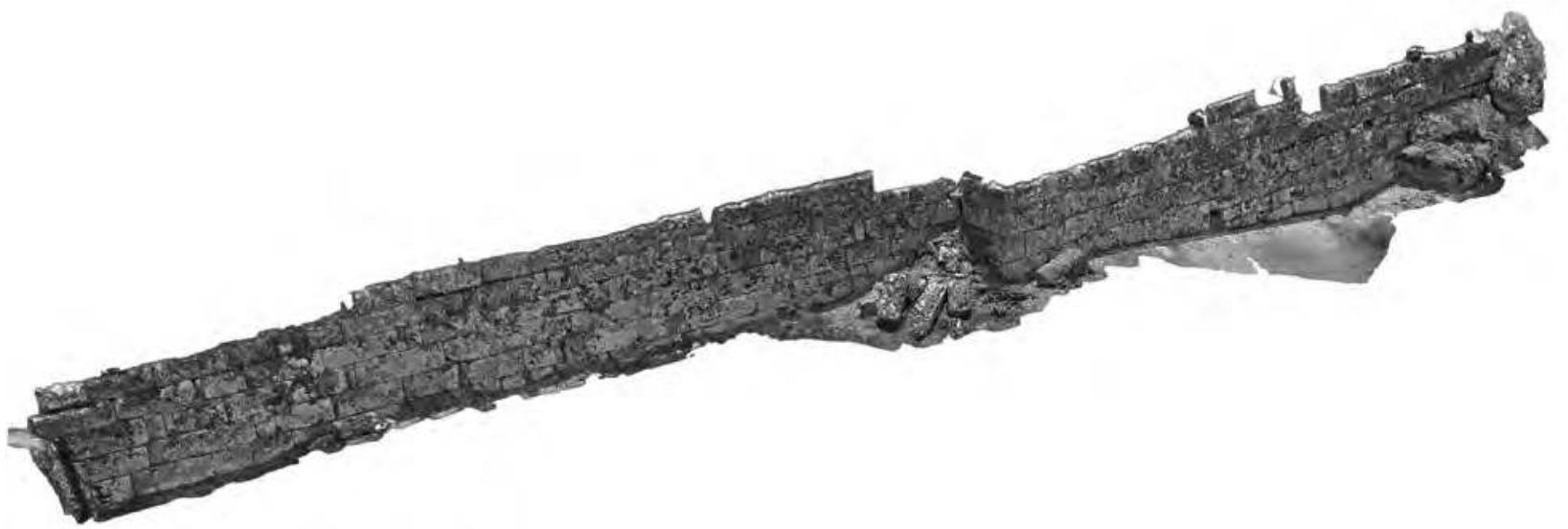

Fig. 2.4.49 : Modèle tri-dimensionnel du mur de fortification de Asarlık Tepe (Fr. Marchand-Beaulieu).

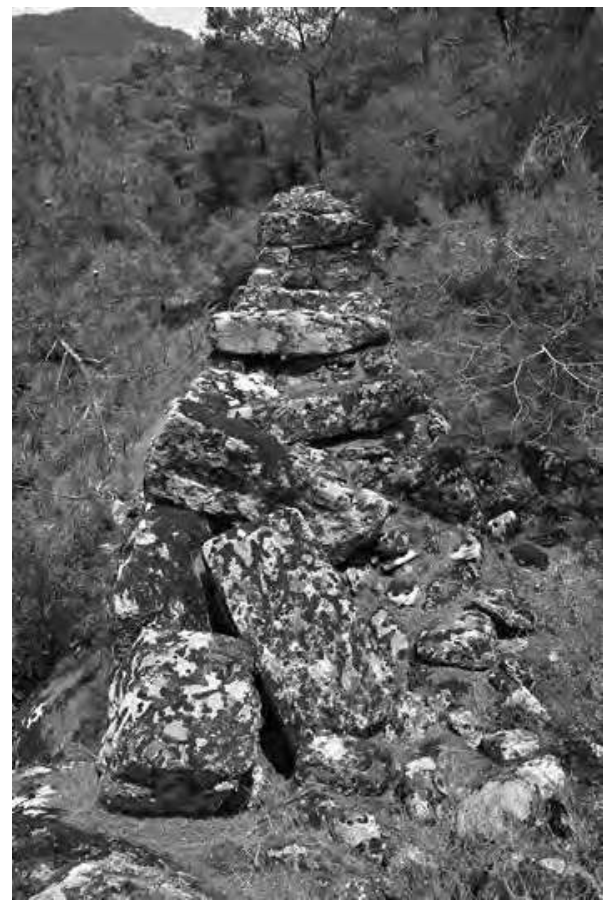

Fig. 2.4.50 : Vue du mur de fortification de Asarlık Tepe depuis le dessus (O. Henry).

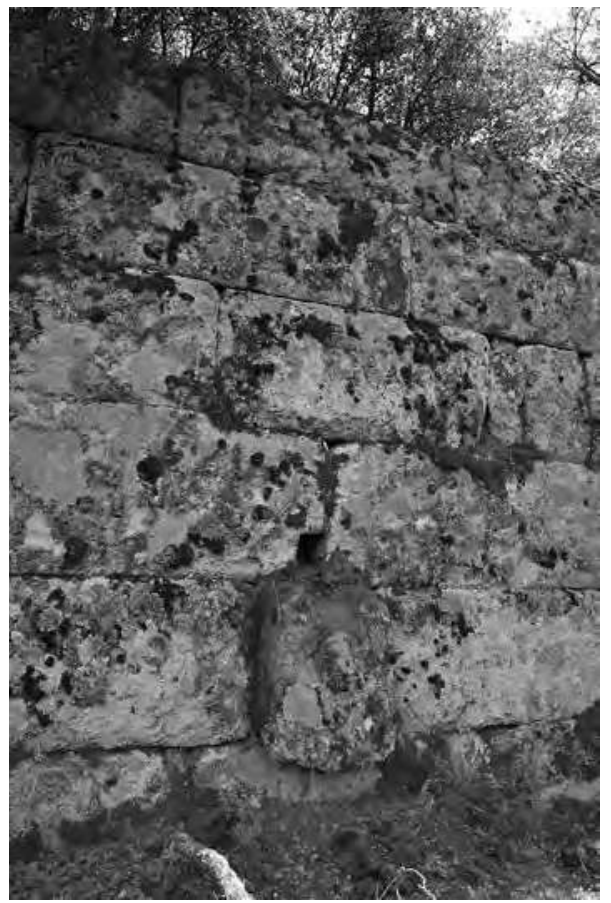

Fig. 2.4.51 : Chantepleure dans le mur de fortification de Asarlık Tepe (O. Henry). 


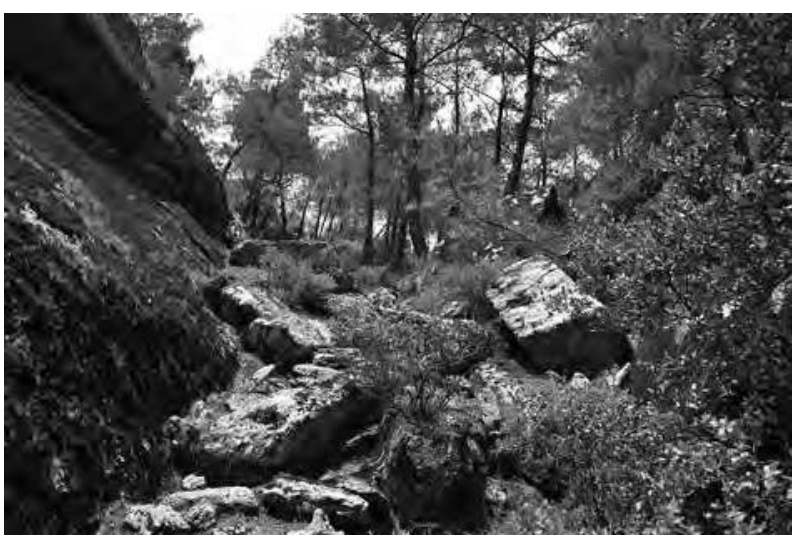

Fig. 2.4.52 : Les vestige de porte ( ?) au pied du mur occidental (O. Henry).

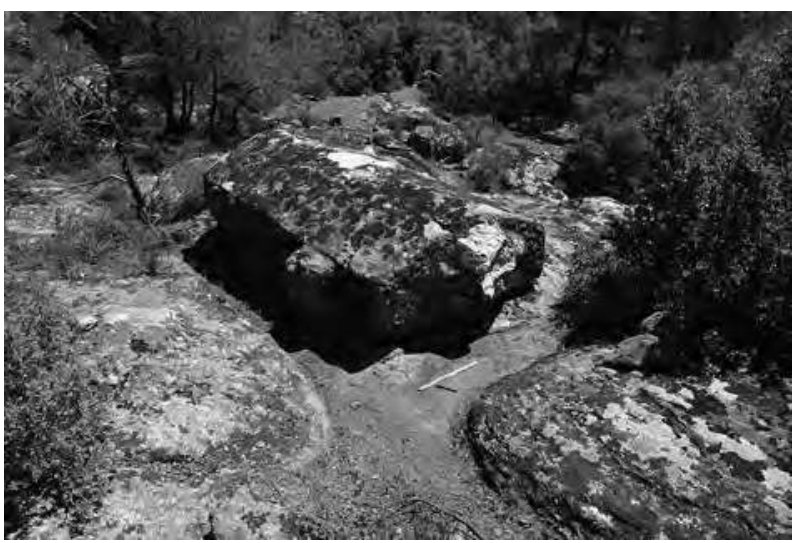

Fig. 2.4.54 : La tombe KOCA001 depuis l'Ouest (O. Henry).

sées sur une longue période de temps. En ce qui concerne la fosse simple 003, sa datation s'avère particulièrement ardue. Néanmoins, la présence des projections sur les faces internes de la fosse pourraient indiquer une tradition partagée avec les sarcophages rupestres de la zone.

\section{B. Les aménagements de production (KOCA010 et KOCA005)}

Le couvercle du sarcophage rupestre de la tombe KOCA010 est en position renversée, à environ $2 \mathrm{~m}$ au Sud-Est de la fosse (Fig. 2.4.562.4.57). Seule sa face inférieure et une partie de ses quatre côtés sont visibles. Les reliefs de préhension sont encore conservés sur les longs côtés latéraux et le petit côté sud. Ces reliefs sont toutefois mal conservés et semblent avoir été retaillés dans une phase ultérieure. Ces tailles secondaires pourraient être liées à une réutilisation spécifique du couvercle dans un contexte non funéraire. La face visible du couvercle a en effet été creusée afin de former un bassin large et peu profond. On re-

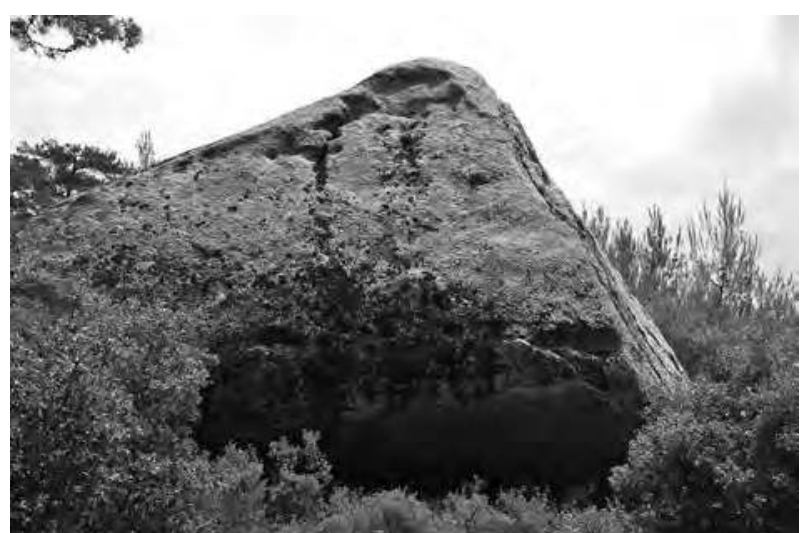

Fig. 2.4.53 : Traces d'une charpente au-dessus d'un abris sous roche à Asarlık Tepe (O. Henry).

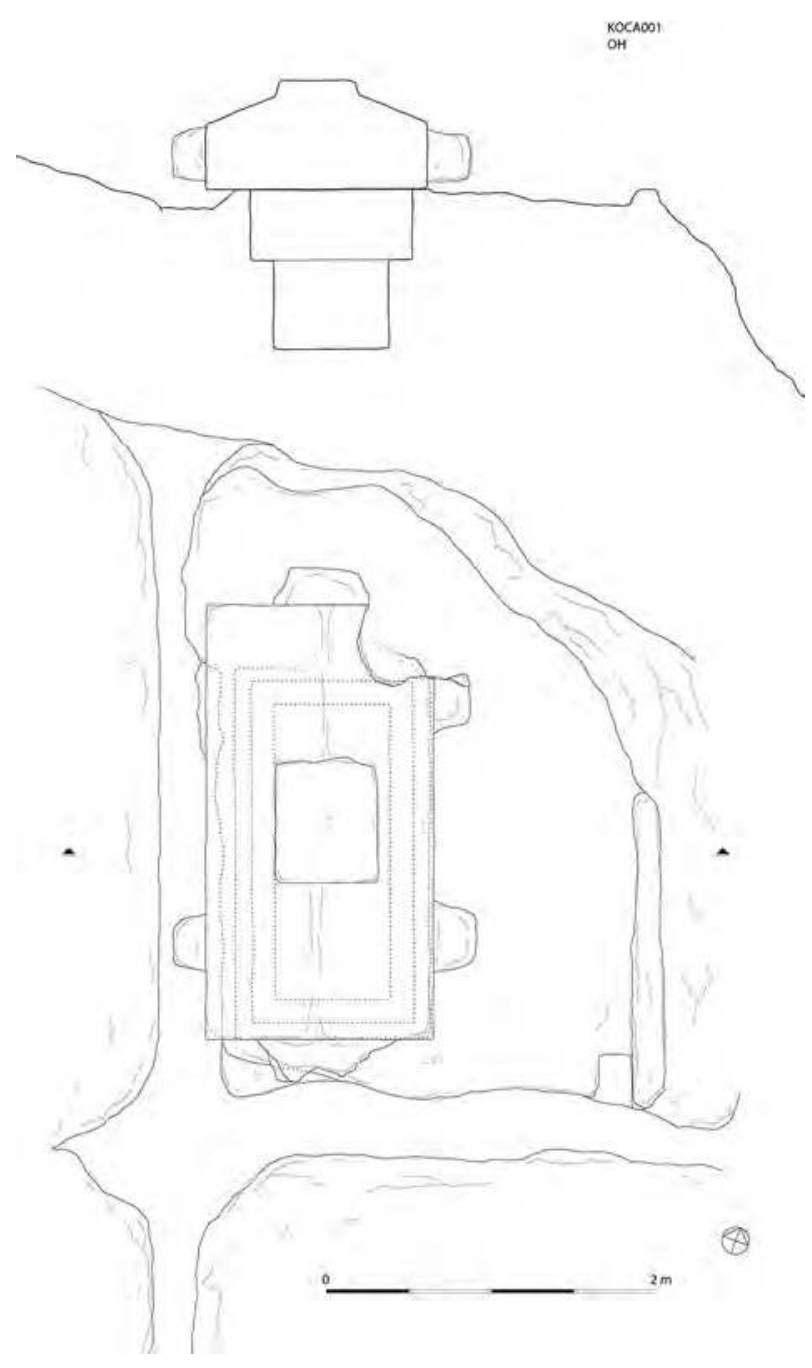

Fig. 2.4.55 : Relevé de la tombe KOCA001 (O. Henry). 


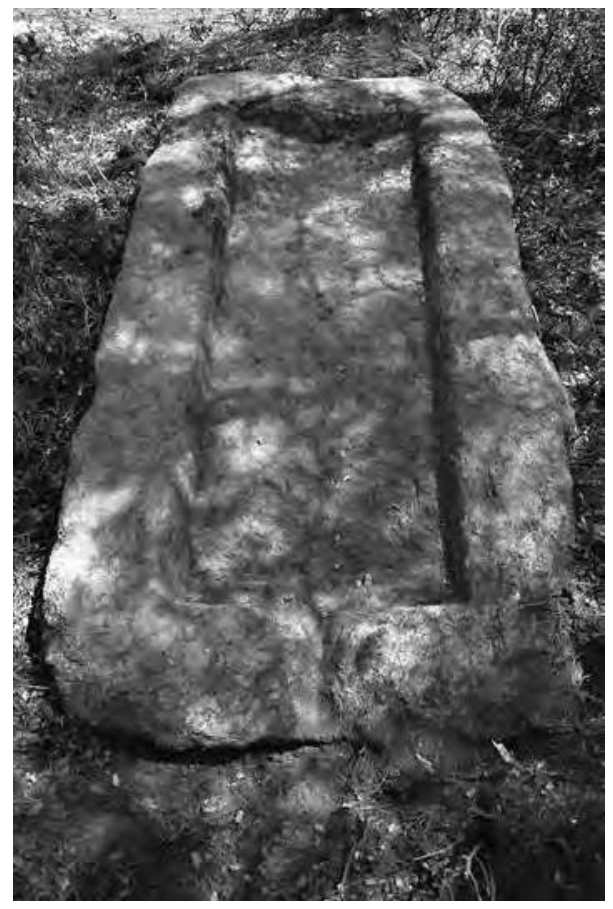

Fig. 2.4.56 : Le couvercle de la tombe KOCA003 (O. Henry).

marque également qu'un sillon de $2 \mathrm{~cm}$ de large perce le bord du bassin creusé, au centre de sa paroi sud. Il est possible que ces aménagements aient visé à transformer le couvercle du sarcophage rupestre en pressoir/fouloir.

Si le couvercle de KOCA010 semble avoir été réutilisé à des fins de production agricole, un autre aménagement découvert à Kocalan confirme que le site abritait au moins une autre production d'huile d'olive ou de vin. Cet aménagement, distinct du précédent puisque situé à plus d'une centaine de mètres, prend la forme d'une très large mortaise rectangulaire pratiquée dans la paroi latérale d'un imposant rocher taillé. Il s'agit d'un aménagement tout à fait typique pour des installations de prelum pour une presse (huile ou vin).

\section{Les terrasses (KOCA009)}

Des vestiges de nombreuses terrasses étroites, peu élevées et très longues ont été repérées à Kocalan. Ces terrasses, qu'il convient de mettre en relation avec les outils de production révélés ci-dessus, sont situés à $100 \mathrm{~m}$ au Nord de KOCA005, plus haut sur la pente douce du site. Leur faible état de conservation ne permet de les observer que sur une surface d'environ $1000 \mathrm{~m}^{2}$, mais il y peu de doute qu'elle devait couvrir un espace bien plus important.

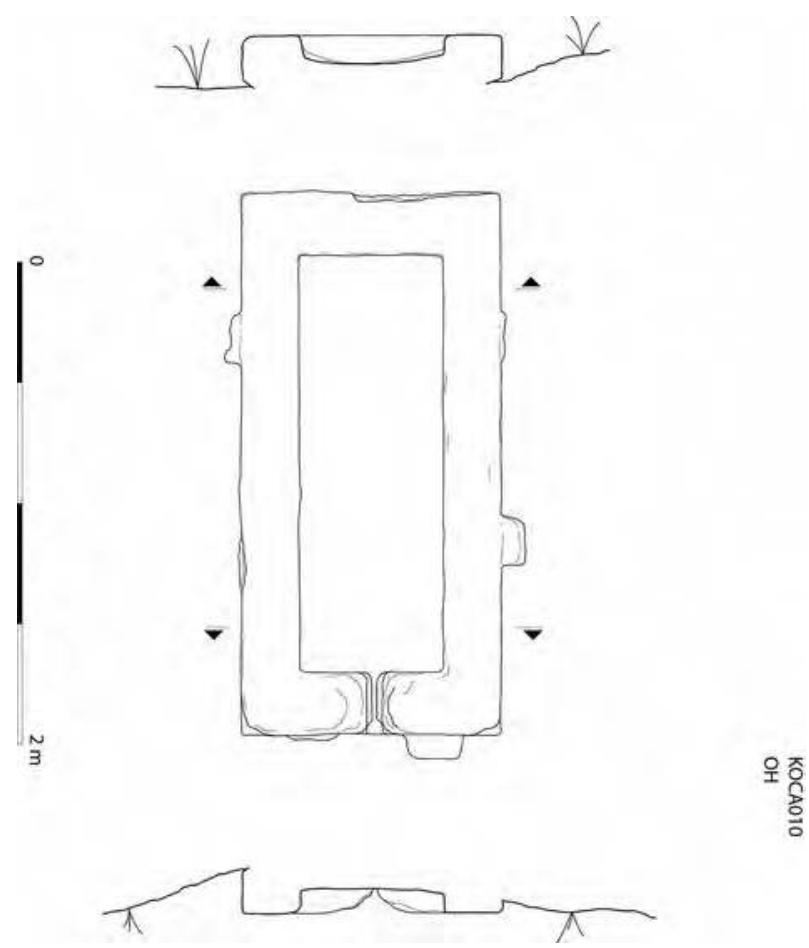

Fig. 2.4.57 : Relevé du couvercle de la tombe KOCA003 (O. Henry).

\section{L'habitation et la citerne (KOCA004, KOCA006)}

Des vestiges qui semblent appartenir à un grand habitat ont été repérés entre les terrasses et la zone de production. Il s'agit d'un groupe de bâtiments carrés, largement détruits (KOCA006) (Fig. 2.4.58). Les murs semblent délimiter un espace rectangulaire, orienté Nord/Sud, de $40 \mathrm{~m}$ de long sur $20 \mathrm{~m}$ de large. L'ensemble est implanté au sommet d'une colline basse et domine le paysage alentour. Immédiatement au-dessous de cet aménagement on trouve une grande citerne, directement taillée dans le sol rocheux (KOCA004). Cette dernière semble avoir une forme oblongue. Elle est encore entièrement inondée et ni son plan ni sa profondeur n'ont pu être calculés.

\section{E. La source aménagée (KOCA008)}

A environ $150 \mathrm{~m}$ au Nord-Ouest des terrasses, se trouve une fontaine aménagée (Fig. 2.4.59). Elle est située à la même altitude que la citerne $(624 \mathrm{~m})$ et il est possible que les deux aménagements aient été reliés par un drain, l'une alimentant l'autre. La fontaine elle-même est encore utilisée et produit un flux constant d'eau claire. De ce fait, il est fort possible que les murs de la fontaine aient été constamment entretenus par les locaux et qu'il ne reste très 
peu du matériel de construction original. Ce type de citerne est très répandu dans les sites anciens de la région.

\section{F. La "tour de garde" naturelle (KOCA007)}

A environ $200 \mathrm{~m}$ à l'Est de KOCA008 et au Nord-Est de KOCA009, bien au-dessus de tous les vestiges repérés à Kocalan, se trouve une formation rocheuse naturelle qui forme un pic étroit mais élevé, atteignant $15 \mathrm{~m}$ au-dessus du niveau du sol (Fig. 2.4.60). Au sommet de ce pic rocheux se trouve un second grand rocher qui offre un abri naturel. Les vestiges d'un mur de protection, construit à l'aide d'un matériel hétéroclite sur le coté de l'abri, indiquent clairement que ce dernier a été occupé. Il ne serait pas surprenant que cet abri rocheux, qui offre une vue dominante sur tout le paysage, fut utilisé comme une sorte de tour de guet.

Aucun matériel associé n'a malheureusement pu nous renseigner sur la période d'utilisation de cet abri sous roche.

\subsubsection{KETENDERE (par O. Henry)}

Quatre sites différents avec des restes anciens ont été repérés dans les limites de Ketendere (à Alınca, Ekinalan, İskele Yaylası et Köseler). Ils sont éloignés les uns des autres et du centre du village lui-même. L'un des sites était déjà connu des autorités locales tandis que l'autre est une nouvelle découverte.

\section{A. Alınca - Fortification / Habitat ?}

A environ $1700 \mathrm{~m}$ au Nord-Est du village de Ketendere, sur l'Alınca Yaylası et sur le Gözdengeç Sr., se trouve une petite formation rocheuse naturelle qui offre à la fois un emplacement naturellement défensif et une position dominante (à une altitude de $735 \mathrm{~m}$ ) sur le paysage environnant (KETEN001) (Fig. 2.4.61). Le site avait été repéré en 2014 par les autorités locales et inscrit sur la liste de protection ( $1^{\mathrm{er}}$ degré) par le conseil régional des monuments historiques. Cette inscription n'a malheureusement pas permis de protéger le site qui est aujourd'hui largement détruit suites à de nombreuses fouilles illégales.

Le site est protégé au Sud, à l'Est et à l'Ouest par des falaises naturelles, tandis que son flanc nord offre une pente douce. La plupart des vestiges semblent s'être trouvés dans cette pente où l'on peut reconnaître de grands blocs équarris ainsi que des restes de murs. Le sommet a été entièrement détruit par les pilleurs et des fragments de céramiques lo- cales en couvrent le sol. Ils n'ont malheureusement pas pu nous donner une chronologie précise du site, mais ils semblent pouvoir être datés dans le courant de l'époque romaine.

\section{B. Ekinalan Sr. - Sarcophage rupestre}

A environ $1600 \mathrm{~m}$ au Nord-Est de Ketendere, au milieu d'une grande clairière sur la pente douce d'Ekinalan Sr., un sarcophage rupestre a été repéré (KETEN002). Il a été taillé au sommet d'un rocher affleurant peu élevé, à environ $1,10 \mathrm{~m}$ au-dessus du sol (Fig. 2.4.62). La fosse rectangulaire mesure $1,86 \mathrm{~m}$ de long sur $74 \mathrm{~cm}$ de large. Elle est orientée approximativement Nord-Est/Sud-Ouest. Ses parois internes portent un ressaut d'environ $10 \mathrm{~cm}$ de large, situé $39 \mathrm{~cm}$ sous l'ouverture. La fosse est partiellement remblayée par de la terre et des petites pierres, nous empêchant d'en observer la profondeur totale. L'ouverture de la fosse est entourée d'un bandeau de $10 \mathrm{~cm}$ de large et de $2 \mathrm{~cm}$ de hauteur. Ce bandeau est lui-même bordé d'un canal sur le long côté sud-est, qui visait à drainer l'eau de pluie. Le couvercle de la tombe a disparu.

Il est assez surprenant de trouver une tombe isolée sans lien apparent avec d'autres éléments qui pourraient révéler une occupation pérenne. Néanmoins, la tombe est située à seulement $1 \mathrm{~km}$ à l'Ouest de KOCA008, et donc assez proche de l'habitat que nous y avons repéré. Les tombes de Ketendere et de Kocalan partagent en outre de nombreuses caractéristiques avec celle-ci et datent probablement de la même période (hellénistique). Dans ce contexte, le tombeau de Ketendere pourrait indiquer l'extension la plus occidentale de l'occupation de Kocalan.

\section{C. İskele Yaylası - Habitat}

L'occupation moderne de İskele Yaylası est située à environ $8 \mathrm{~km}$ au Nord de Ketendere, sur le versant sud de Burklıbelen tepesi à 650-700 m d'altitude. Elle forme un petit village encore habité. Dans le village, on peut remarquer de nombreux vestiges. Parmi les plus anciens, probablement hellénistiques, se trouvent trois sarcophages rupestres, une presse, plusieurs meules, des fontaines aménagées et quelques traces de carrières de pierre.

\section{Les tombes (ISK001, ISK002, ISK003)}

Les trois tombes que nous avons découvertes à İskele Yaylası sont toutes situées à une courte distance l'une de l'autre. Elles semblent avoir fait partie d'une 


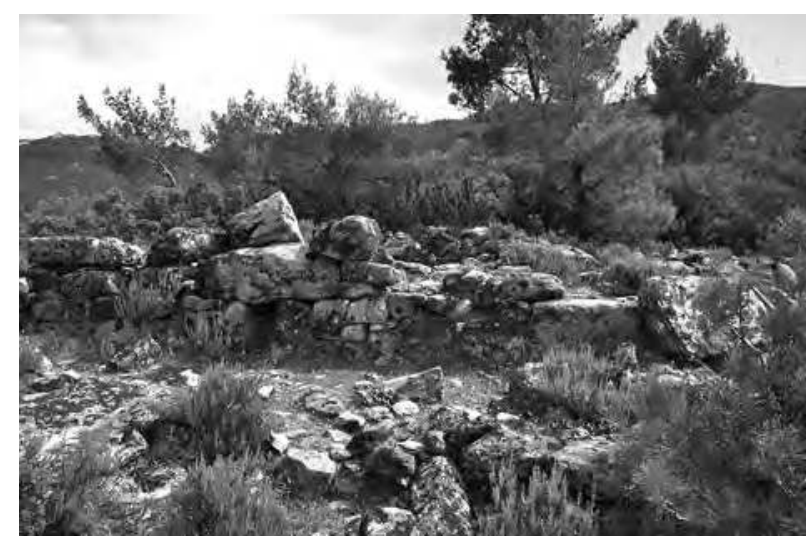

Fig. 2.4.58 : Vestiges de l'habitat à Kocalan (KOCA006) (O. Henry).

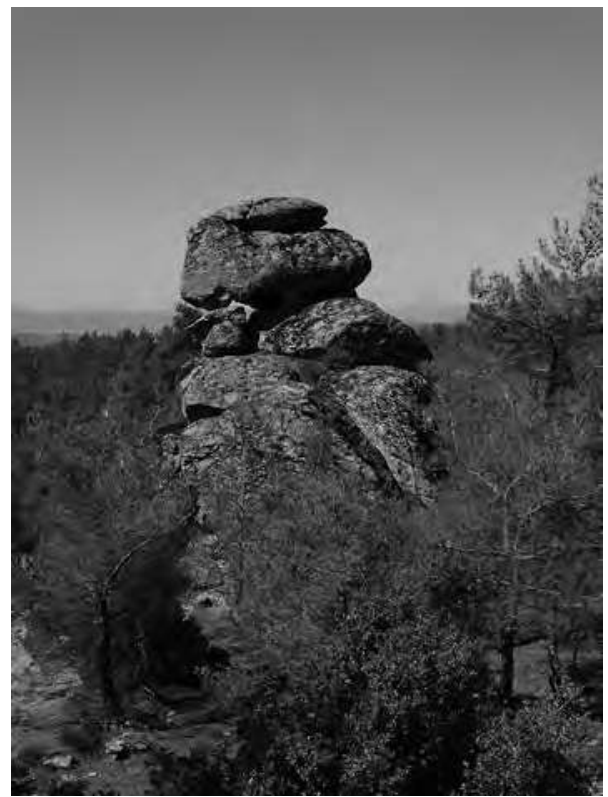

Fig. 2.4.60 : La tour de guet ( ?) à Kocalan (KOCA007) (O. Henry).

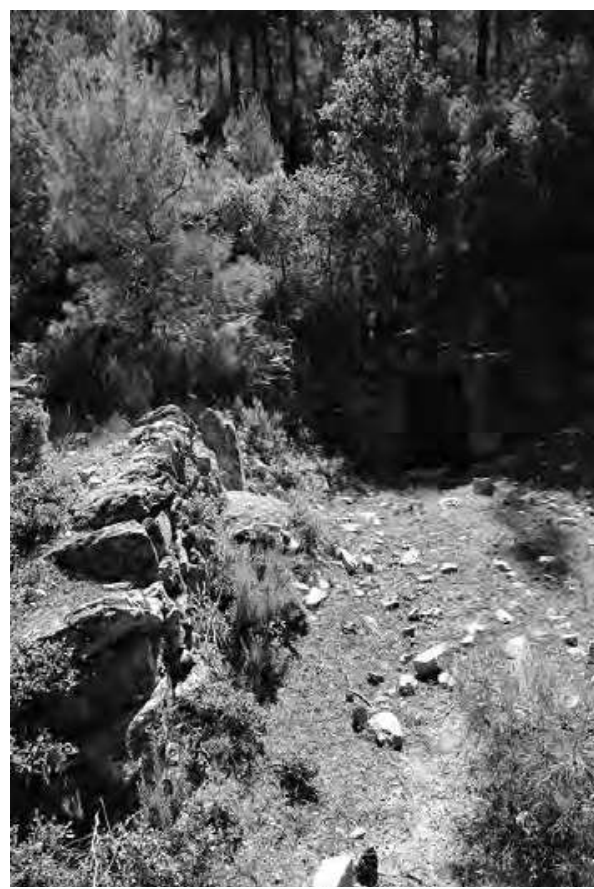

Fig. 2.4.59 : La fontaine aménagée à Kocalan (KOCA008) (O. Henry).

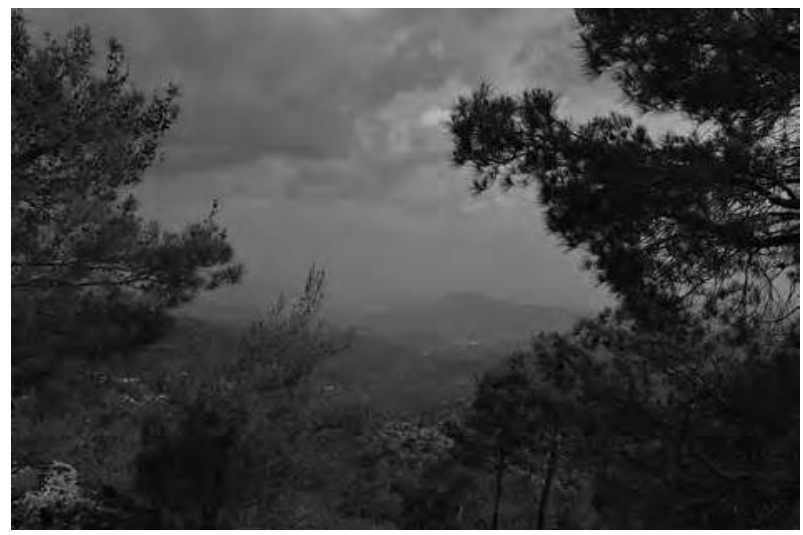

Fig. 2.4.61 : Vue sur la vallée de Milas depuis le site de Alınca (O. Henry).

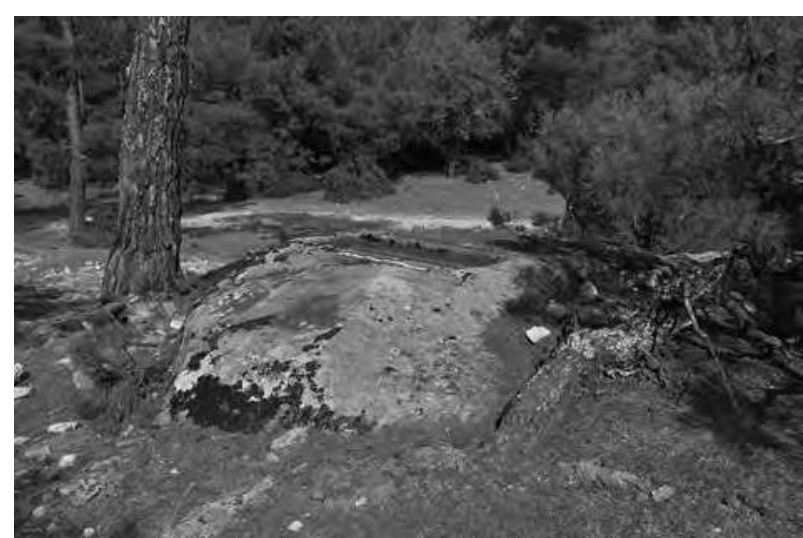

Fig. 2.4.62 : Sarcophage rupestre de Ekinalan Sr. (O. Henry). 


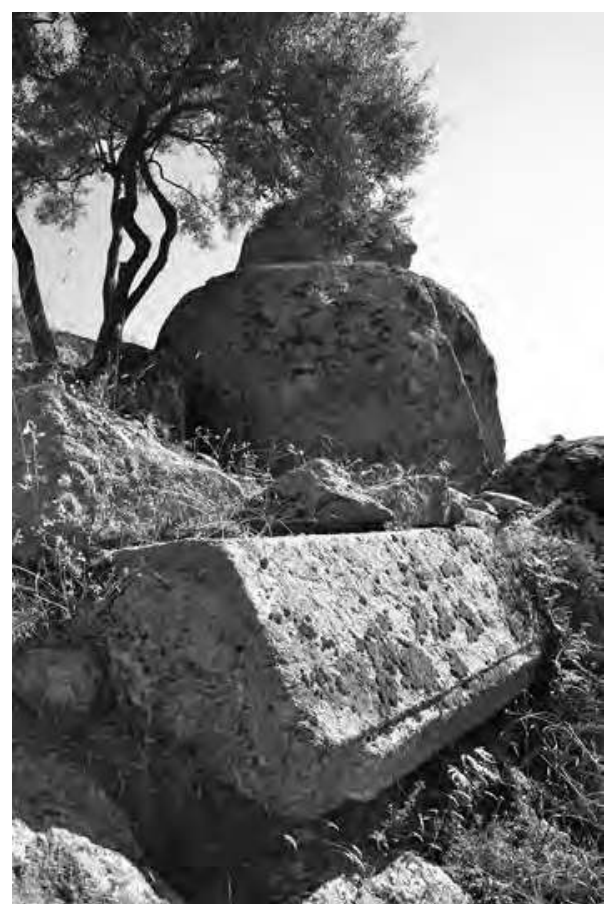

Fig. 2.4.63 : Couvercle de la tombe ISK001, la tombe elle-même se situe au sommet du rocher à l'arrière plan $(\mathrm{O}$. Henry).

nécropole dans la zone méridionale de l'occupation. Toutes les tombes repérées appartiennent au même type des sarcophages rupestres.

\section{ISK001 (Fig. 2.4.63)}

Ce tombeau est sculpté au sommet d'un rocher élevé et étroit. La partie supérieure du rocher a été aplanie afin de créer une plate-forme haute $(3,40$ $\mathrm{m}$ au-dessus du sol à l'Est) au milieu de laquelle la fosse de la tombe a été creusée. Cette fosse mesure 2,00 m de long et $84 \mathrm{~cm}$ de large et est orientée Nord/Sud. Ses parois internes latérales montrent un ressaut de $6 \mathrm{~cm}$ de large, $12 \mathrm{~cm}$ sous le bord de la cuve. La fosse a une profondeur totale de $82 \mathrm{~cm}$. L'ouverture de la fosse est entourée d'un bandeau irrégulier de $28 \mathrm{~cm}$ de large et de $2 \mathrm{~cm}$ de hauteur, partiellement détruit vers le Nord. Le couvercle de la tombe a été déplacé. Il est maintenant couché sur le sol, à 1,50 m au Nord-Est et au pied du rocher portant le tombeau. Il a été réutilisé dans un mur de terrasse et n'est que partiellement visible. C'est un couvercle massif à double pente de $2,40 \mathrm{~m}$ de longueur, 1,32 $\mathrm{m}$ de large et $68 \mathrm{~cm}$ d'épaisseur à son faîte. Son poids est d'environ 4 tonnes. Chacun des quatre côtés du couvercle est orné de bosses de préhension : sur les petits côtés, ces derniers mesurent

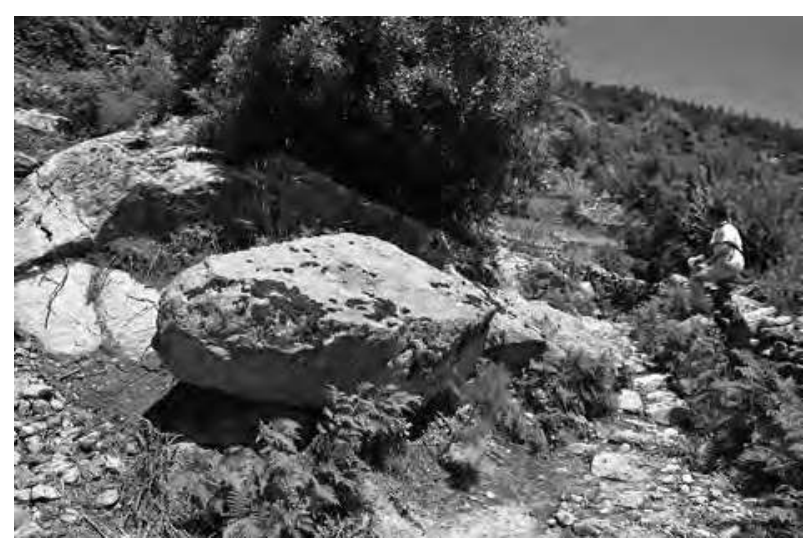

Fig. 2.4.64 : Extraction d'un bloc monumental à İskele Yaylası (O. Henry).

$60 \mathrm{~cm}$ de large, et projettent sur $17 \mathrm{~cm}$, tandis que sur chacun des cotés longs il s'agit d'un long (1,92 $\mathrm{m}$ ) et étroit bandeau, de $19 \mathrm{~cm}$ de hauteur, projetant sur $5 \mathrm{~cm}$, de section rectangulaire.

\section{ISKOO2}

La tombe est située à $10 \mathrm{~m}$ au Sud-Est, plus bas sur la pente, sous ISK001. Elle est aménagée sur un affleurant rocheux peu marqué, dont le sommet ne dépasse du sol que de quelques centimètres. Cette tombe n'est pas seulement mal conservée mais sa cuve est aussi entièrement remplie de petites pierres, ce qui limite les observations. La tombe est orientée Nord/Sud. Elle a une longueur de 1,80 m et une largeur de 60 à $64 \mathrm{~cm}$. Sa section semble irrégulière. On ne peut pas remarquer si les parois latérales de la fosse disposent d'un ressaut et la mauvaise conservation de la roche interdit même de constater la présence d'un bandeau ceinturant l'ouverture de la fosse. Le couvercle de la tombe a disparu.

\section{ISKO03}

La tombe est située à $20 \mathrm{~m}$ au Nord d'ISK001, au pied du mur de pierre moderne limitant la parcelle de terrain contenant à la fois ISK001 et ISK002. L'affleurement rocheux dans lequel la fosse a été aménagée est étroit et peu élevé. La roche est sérieusement endommagée et donne l'impression que la cuve n'a pas été soigneusement taillée. La plateforme dans laquelle la cuve fut taillée est étroite et accommode à peine la cuve elle-même. Cette dernière est rectangulaire, orientée Nord/Sud. Elle mesure $1,84 \mathrm{~m}$ de long sur $64 \mathrm{~cm}$ de large. Le fond de la cuve est remblayé jusqu'à $34 \mathrm{~cm}$ sous le bord. Aucun ressaut sur les parois internes n'est visible. Il est possible qu'un tel aménagement n'ait jamais 
ISK005

$\mathrm{OH}$ - Sketch drawing
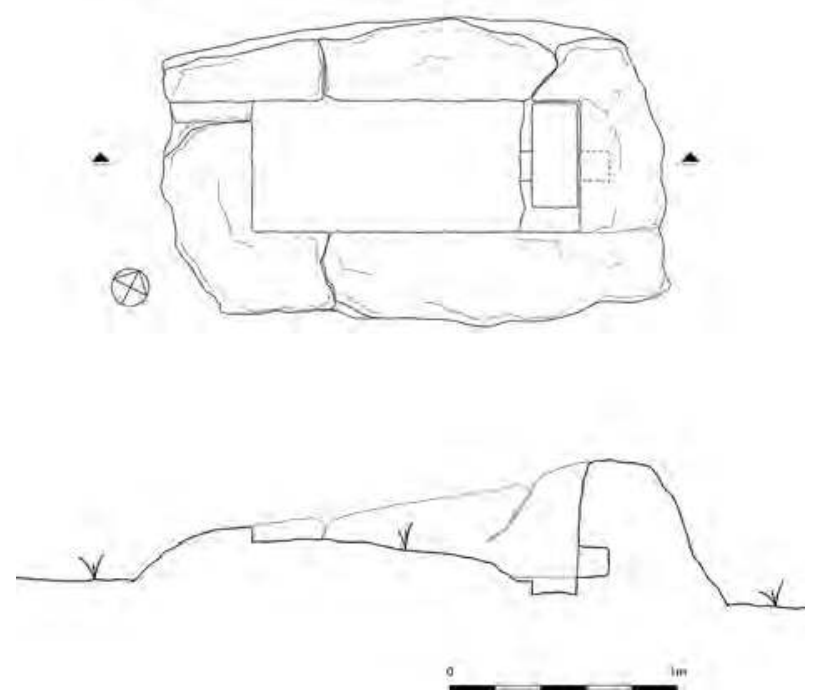

Fig. 2.4.65 : Une cuve de presse/fouloir (?) à İskele Yaylası (O. Henry).

été taillé, ce qui serait assez inhabituel. Il est aussi probable que le ressaut, situé en position basse, soit caché par le remblai (en KOCA001, le ressaut est situé $42 \mathrm{~cm}$ sous le bord de la cuve). Le couvercle de la tombe a disparu.

\section{Les traces de taille}

A plusieurs endroits dans le village, nous avons remarqué des traces d'extraction, impliquant parfois de très grands blocs de roche (Fig. 2.4.64). Les techniques d'extraction ne semblent pas avoir utilisé la technique de rainure de préparation. Au lieu de cela, il semble que les carrières utilisent de petites mortaises placées l'une à côté de l'autre, dans lesquelles étaient insérés des coins en bois. Cette technique a été utilisée pendant des siècles, depuis l'antiquité jusqu'à très récemment, et il est extrêmement difficile de dater de tels vestiges. Cependant, on remarque qu'aucune des maisons du village moderne utilise de larges blocs de taille, il est donc très probable que cette activité de carrière date d'une période ultérieure à l'établissement du village moderne.

\section{Une presse (?)}

Plus haut dans le village, nous avons découvert un aménagement ancien que nous avions d'abord interprété comme la cuve d'une tombe rupestre (ISK005). Cet aménagement est constitué d'une fosse rectangulaire $(1,44 \mathrm{~m}$ sur $58 \mathrm{~cm})$, taillée dans un affleurement rocheux peu élevé, et orientée NordEst/Sud-Ouest. La cuve n'est néanmoins pas placée au centre d'une plate-forme taillée horizontalement, mais plutôt sur un des côtés du rocher affleurant. En conséquence, chacun des quatre côtés de l'ouverture de la cuve se situent à une hauteur distincte, ce qui interdit de fermer la fosse avec un couvercle horizontal. Un nettoyage de surface de la fosse a révélé des caractéristiques inhabituelles : la cuve est relativement peu profonde $(65 \mathrm{~cm})$; une mortaise carrée de $13 \mathrm{~cm}$ de côté et $14 \mathrm{~cm}$ de profondeur a été taillée dans la paroi est, $52 \mathrm{~cm}$ sous le sommet de la cuve; sous la mortaise, une cuve secondaire décalée vers le Nord ( $20 \times 43 \mathrm{~cm}$, profondeur inconnue) a été creusée ; sur le côté ouest de cette cavité secondaire, on remarque l'aménagement d'un canal de $13 \mathrm{~cm}$ de large et $2 \mathrm{~cm}$ de profondeur qui visait apparemment à drainer un liquide depuis le corps principal de la cuve principale vers la cuve secondaire (Fig. 2.4.65). La fosse est encore largement remblayée et aucune autre observation supplémentaire ne peut être faite. Il semble néanmoins que ISK005 ne puisse pas être identifiée à une sépulture, mais peut-être à un dispositif utilisé pour la production d'un liquide, soit de l'huile d'olive, soit du vin. La mortaise dans le mur Est aurait pu servir d'encoche pour un prelum de petite section, alors que la cavité secondaire aurait pu servir de conteneur pour le moût (de raisin ?).

\section{Habitat (?)}

Parmi les terrasses modernes, certaines parties de murs semblent plus anciennes que d'autres. On remarque par ailleurs que de larges parpaings sont encore conservés, notamment des blocs longs et étroits, dressés, qui semblent former les deux jambages d'une porte reposant sur un troisième bloc, horizontal (seuil ?) (ISK006).

Fontaines (ISK007, ISK008, ISK009) (Fig. 2.4.66)

Parmi les nombreux vestiges repérés à İskele Yaylası, il faut souligner la très grande quantité de fontaines aménagées (plus d'une trentaine au bas mot). Trois d'entre elles, parmi la trentaine de fontaines repérées, ont été documentées. Comme indiqué plus haut, ces fontaines sont souvent aménagées dans un premier temps à l'époque antique et sont, ensuite, entretenues par la population locale. Ces fontaines d'İskele Yaylası offrent des caractéristiques variées. La plupart d'entre elles sont précédées d'un long dromos, généralement un indice d'ancienneté. Néanmoins, comme elle demeurent 


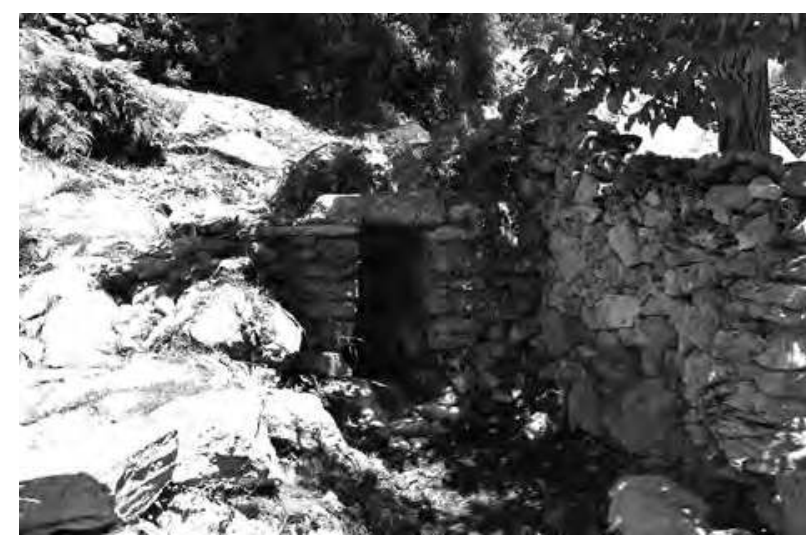

Fig. 2.4.66 : Un exemple des nombreuses fontaines aménagées à İskele Yaylası (O. Henry).

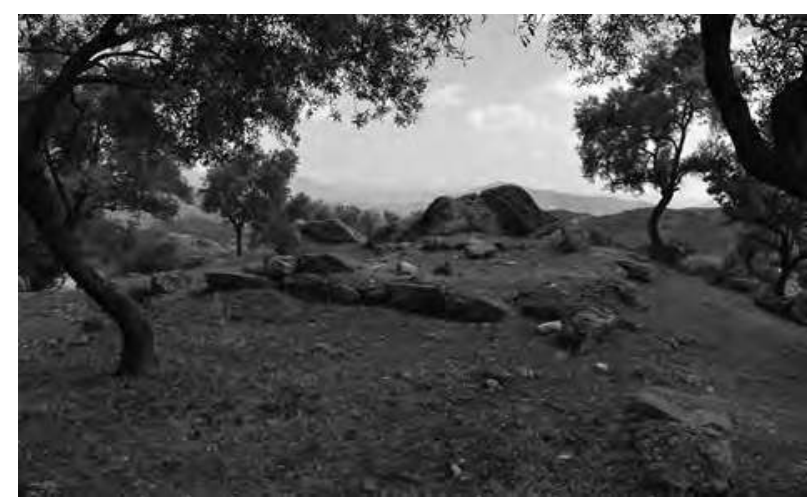

Fig. 2.4.68 : Vestiges d'habitat à Köseler (KOSE001-D) (O. Henry).

encore en fonction et ont probablement bénéficié d'un soin constant de la part des locaux, il reste difficile de déterminer ce qui reste du bâtiment d'origine. L'abondance de l'eau pourrait facilement expliquer les raisons de l'implantation d'une communauté dès l'antiquité.

\section{Pierres de meule}

Dans le village, nous avons repéré plusieurs pierres de meule, indiquant l'existence d'une importante production de blé et de farine. Une de ces meules a été documentée. Elle a été taillée dans un bloc de gneiss local. Elle mesure $42 \mathrm{~cm}$ de diamètre. L'une de ses faces est plate tandis que l'autre est convexe (Fig. 2.4.67). Au centre de la face convexe, une petite mortaise rectangulaire $(3 \times 4 \mathrm{~cm})$ a été taillée, tandis que le centre de la face plate présente une cavité hémicirculaire de $12 \mathrm{~cm}$ de diamètre.

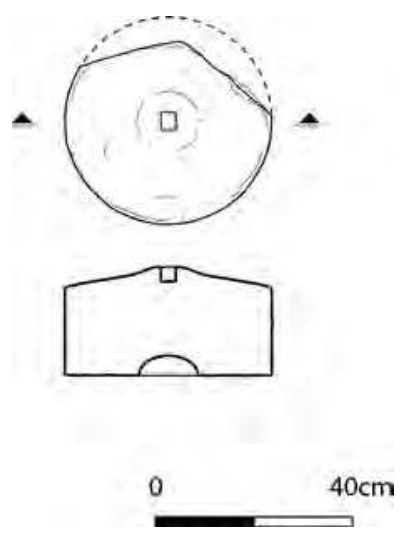

Fig. 2.4.67 : Pierre de meule à İskele Yaylası (O. Henry).

\section{Köseler - Habitat}

Le site de Köseler est situé à environ $750 \mathrm{~m}$ au Sud-Ouest du village de Ketendere. Il se trouve sur la pente sud du Gemikaya tepe, à une altitude de 350-380 m. Les vestiges de Köseler indiquent la présence d'une occupation modeste, non fortifiée mais placée dans une position dominante sur le paysage. L'emplacement semble avoir été soigneusement choisi car il est l'un des rares endroits où la topographie naturelle forme une zone plane, qui projette depuis la pente vers le Sud. La production de blé/ farine et d'huile d'olive semble avoir été l'une des principales activités de cette petite communauté.

\section{Habitat}

Au moins deux complexes de bâtiments sont repérables. L'un (KOSE001-A) est situé sur le point le plus haut de la zone, composé de plusieurs murs en matériau hétérogène mélangeant à la fois moellons et grands blocs. Il est de plan rectangulaire, orienté Nord/Sud et couvre une superficie d'environ $200 \mathrm{~m}^{2}$ (20 par $10 \mathrm{~m}$ ). Le deuxième complexe est situé légèrement plus bas sur la pente vers l'Est, au sommet d'une petite éminence (KOSE001-D) (Fig. 2.4.68). Ici, le plan est plus clair. Il se compose de deux pièces adjacentes et forme un bâtiment allongé orienté Nord/Sud. Les deux pièces ont les mêmes dimensions $(4 \times 5 \mathrm{~m})$ mais sont orientées perpendiculairement l'une à l'autre. Les murs, construits en grand appareil, sont mal conservés.

\section{Structures de production}

Les autres vestiges repérés sur le site concernent principalement des aménagements de production. A KOSE001-B, nous avons repéré à quelques mètres 


$$
\text { KOSEOO1-B }
$$

$\mathrm{OH}$
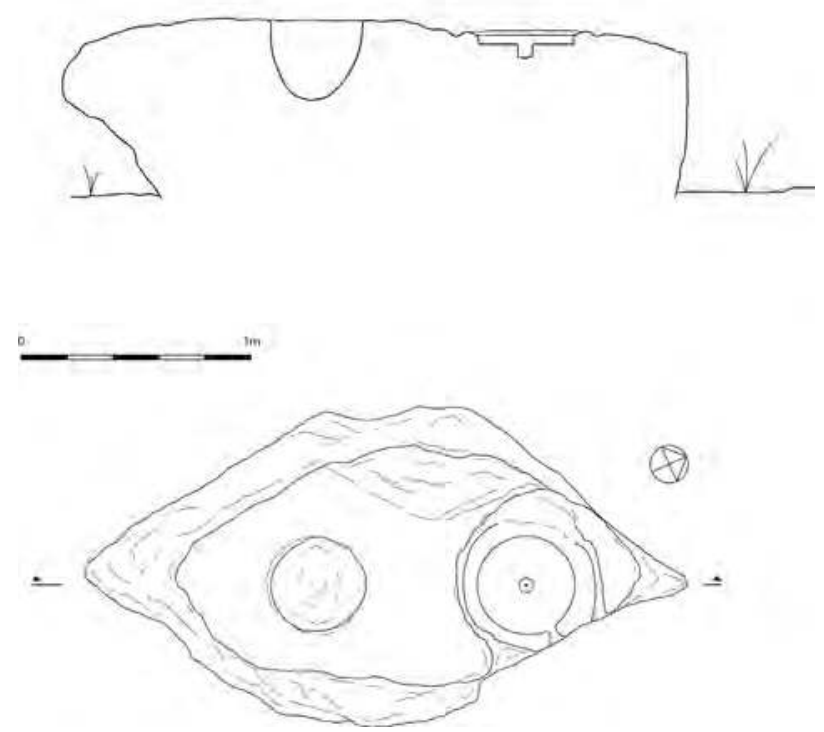

Fig. 2.4.69 : Meule et mortier à Köseler (KOSE001-A)(O. Henry).

au Nord de KOSE001-A des aménagements taillés sur la surface supérieure aplanie d'un rocher peu élevé (Fig. 2.4.69). Il s'agit de deux cavités voisines l'une de l'autre. Au Sud, on trouve une cavité circulaire de $40 \mathrm{~cm}$ de diamètre et $34 \mathrm{~cm}$ de profondeur, à la section hémisphérique. Il est probable qu'il s'agisse d'un mortier à céréales. Une telle identification est confirmée par les caractéristiques de la seconde cavité, une meule à grain. Celle-ci est parfaitement circulaire, d'un diamètre de $42 \mathrm{~cm}$, peu profonde $(6 \mathrm{~cm})$ avec une paroi latérale parfaitement verticale sur $4 \mathrm{~cm}$. Un canal horizontal creusé dans la paroi latérale orientale permettait de verser la farine produite vers un récipient placé au contact du rocher. Une mortaise circulaire, de $6 \mathrm{~cm}$ de diamètre et $6 \mathrm{~cm}$ de profondeur, a été taillée au centre de la meule afin de sécuriser l'axe de rotation de la partie mobile. L'axe, probablement en bois, avait une extrémité inférieure pointue, placée dans une deuxième cavité, minuscule, située au centre de la mortaise circulaire.

La production de farine n'était pas la seule activité de cette communauté, qui semble également avoir produit de l'huile d'olive si l'on en croit la pierre de presse découverte à $100 \mathrm{~m}$ au Nord-Est de la meule, plus haut sur la pente (Fig. 2.4.70). La presse (KOSE001-C) a été aménagée au sommet $(60 \mathrm{~cm}$ au-dessus du sol) d'une grande pierre aplanie, dont on a aussi taillé les parois verticales afin qu'elles adoptent le plan de la presse. Ce dernier est de forme ovoïde, allongé et pointu, et mesure environ 1,20 m

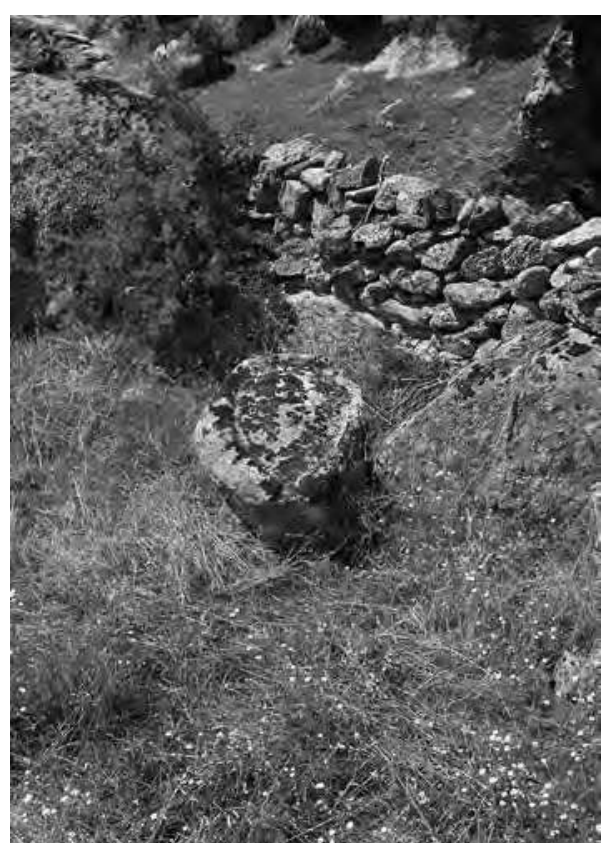

Fig. 2.4.70 : Pierre de maie à Köseler (KOSE001-C) (O. Henry).

de long sur $86 \mathrm{~cm}$ au plus large. La surface centrale est cernée par une rigole profonde et peu large qui ouvre sur un canal verseur pratiqué à l'extrémité pointue de la pierre. La surface de la presse n'est pas horizontale mais légèrement inclinée vers le bec verseur. Il est impossible de déterminer si cette pente était intentionnelle ou non.

Plus à l'Est, nous avons repéré dans un champ une colonnette sculptée à partir d'une pierre de gneiss local (KOSE001-E). Elle mesure $70 \mathrm{~cm}$ de long et $31 \mathrm{~cm}$ de diamètre et une de ses extrémités est percée d'une mortaise circulaire, de $5 \mathrm{~cm}$ de diamètre et $3,5 \mathrm{~cm}$ de profondeur. Les extrémités de la colonnette semblent très érodées. De telles caractéristiques semblent indiquer qu'il s'agit là d'un broyeur, probablement destiné à la production d'huile d'olive.

A proximité de la colonnette, nous avons découvert un deuxième mortier creusé au sommet d'un rocher naturel (KOSE001-F). Il est plus large mais moins profond que le premier avec $50 \mathrm{~cm}$ de diamètre et $15 \mathrm{~cm}$ de profondeur. A proximité de ce mortier, on trouve, dans un mur de terrasse, une pierre soigneusement taillée, de forme hémisphérique. Elle mesure $30 \mathrm{~cm}$ de diamètre et 30 $\mathrm{cm}$ d'épaisseur. Elle dispose, sur sa surface plane, d'une mortaise décentrée. La forme hémisphérique de la pierre et la mortaise non centrée, qui aurait pu être utilisée pour fixer une perche, pourrait indiquer qu'il s'agit ici de la partie mobile du mortier rupestre repéré à proximité. 
Aucun fragment de céramique n'a pu être récolté sur ce site et nous ne sommes donc malheureusement pas en mesure de donner un cadre chronologique à son occupation qui semble toutefois antique (période romaine ? basée sur la construction des murs de l'habitat). Il est surprenant qu'aucune tombe n'ait été repérée sur place. Ceci pourrait indiquer qu'il s'agit probablement d'un habitat temporaire, lié aux activités de production. Il est aussi possible que des tombes soient présentes ici mais qu'elles n'aient jamais été découvertes par les pilleurs dont l'activité semble avoir été néanmoins particulièrement intense dans la zone.

\subsection{4. İKIZZTAŞ (par O. Henry)}

Nous avons étudié quatre sites localisés dans les limites du district du village d'İkiztaş. Trois d'entre eux, s'ils n'avaient jamais fait l'objet d'une documentation, étaient déjà connus des autorités locales.

Le premier site s'étend autour, au pied et à proximité immédiate des deux grands pics rocheux qui ont donné leur nom au village (ikiz = jumeau, taş $=$ rocher). Il comprend les vestiges numérotés de IKIZ001 à IKIZ013. Le deuxième site est situé le long de la route principale menant à İkiztaş, à $750 \mathrm{~m}$ au Sud du village (IKIZ014 et IKIZ020). Les deux sites avaient été reconnus en 2010 et inscrits sur la liste de protection par décision du conseil régional des monuments historiques. Le troisième site est situé à $500 \mathrm{~m}$ à l'Est de l'école du village (IKIZ015IKIZ016). Il avait également été identifié par les autorités locales (en 2014) et placé sur la liste des sites protégés. Le quatrième et dernier site, inconnu auparavant, est situé plus à l'Est et plus haut sur la pente, vers Alınca Yaylas1, à environ 1 km de l'école du village (IKIZ017-IKIZ018).

Même si peu de fragments céramique ont pu être récoltés au cours de la prospection, les vestiges indiquent clairement une occupation aux époques hellénistique tardive et romaine.

\section{A. İkiztaş tepesi - Fortification et habitat}

Même si les vestiges repérés occupent deux zones distinctes, il est difficile de les considérer séparément l'un de l'autre. Les vestiges concernés sont géographiquement déconnectés, l'un prenant place immédiatement au pied des pics rocheux, tandis que l'autre est situé quelques $250 \mathrm{~m}$ à l'Ouest de ces derniers. Cependant, ils partagent beaucoup de caractéristiques qui indiquent une période d'occupation commune. Il est possible que l'occupation sur et autour des sommets ait appartenu à une période "haute" de l'occupation de la zone, avec une puissante fortification utilisant les pics rocheux euxmêmes, entourés d'une nécropole. Le deuxième groupe de vestiges pourrait dès lors appartenir à l'expansion tardive de cette même occupation, avec une zone funéraire élargie ainsi que des traces d'installation urbaine.

\section{Le voisinage immédiat des sommets}

İkiztaş est composé de deux pics imposants, dont le plus élevé est au Sud, séparés par un espace en selle de cheval d'environ $100 \mathrm{~m}$ de long et $50 \mathrm{~m}$ de large. Ces pics créent un repère impressionnant dans le paysage, ainsi qu'une position naturellement inexpugnable avec de hautes falaises reliant les deux pics à l'Est (Fig. 2.4.71).

La prospection menée entre les pics ainsi que sur le versant ouest a révélé une occupation relativement dense. Les constructions principales incluent de grands murs, construits aux pieds des pics (IKIZ007) et les reliant l'un à l'autre. Composés d'un blocage de moellons scellés au mortier, ces murs ont une largeur de 1,60 m et semblent fermer le versant occidental de la double formation rocheuse (Fig. 2.4.72). Vers le Sud, le mur longe littéralement la face ouest du pic rocheux, créant ainsi une sorte de long corridor étroit. Plus au Nord, le mur dont l'élévation peut atteindre jusqu'à $2 \mathrm{~m}$ de hauteur relie les deux pics. A l'intérieur de l'espace dessiné d'une part par ces murs à l'Ouest et d'autre part par les falaises à l'Est, la végétation est très dense et presque aucune autre structure ne peut être repérée. Néanmoins, de nombreux fragments de céramique, des scories métalliques et de fragments de tuiles et de briques insérées dans une gangue de mortier indiquent que l'occupation a probablement débuté dès la période romaine, sinon plus tôt. Immédiatement au-dessous du mur occidental, on remarque de nombreux vestiges de terrasses anciennes. Beaucoup d'entre-elles se sont effondrées, mais la quantité de pierres empilées semble indiquer une importante élévation dans leur état original. Dans deux cas, ces murs de terrasse sont associés à des seuils directement découpés dans la roche (IKIZ005, Fig. 2.4.73 et IKIZ006), révélant la présence d'un habitat qui occupait probablement une grande partie de la pente ouest. Ces seuils sont maintenant situés très au-dessus du sol, à parfois près de $2 \mathrm{~m}$, ce qui confirme l'impression selon laquelle les murs de terrasse pouvaient atteindre d'importantes élévations.

Plusieurs vestiges funéraires ont été repérés environ $30 \mathrm{~m}$ plus bas dans la pente, à l'Ouest du pic nord. Deux sarcophages rupestres ont été aménagés à la surface d'un affleurement rocheux. Le premier 


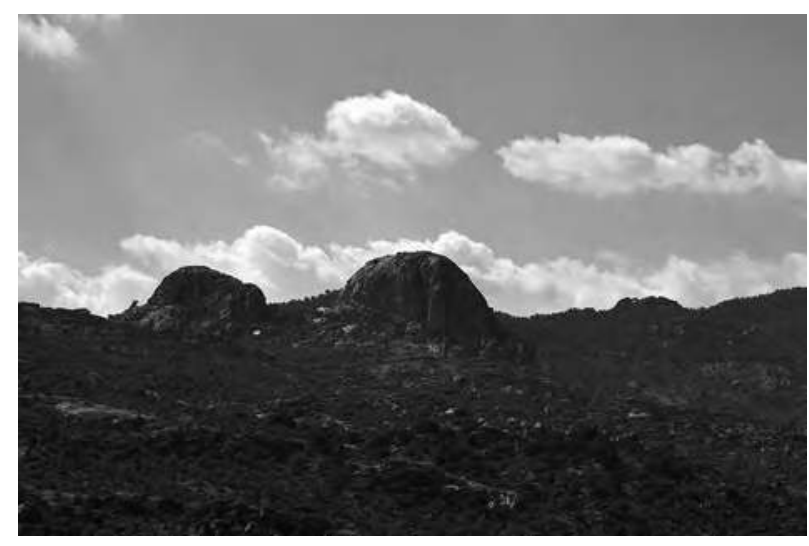

Fig. 2.4.71 : Vue de İkiztaş depuis l'Est (O. Henry).

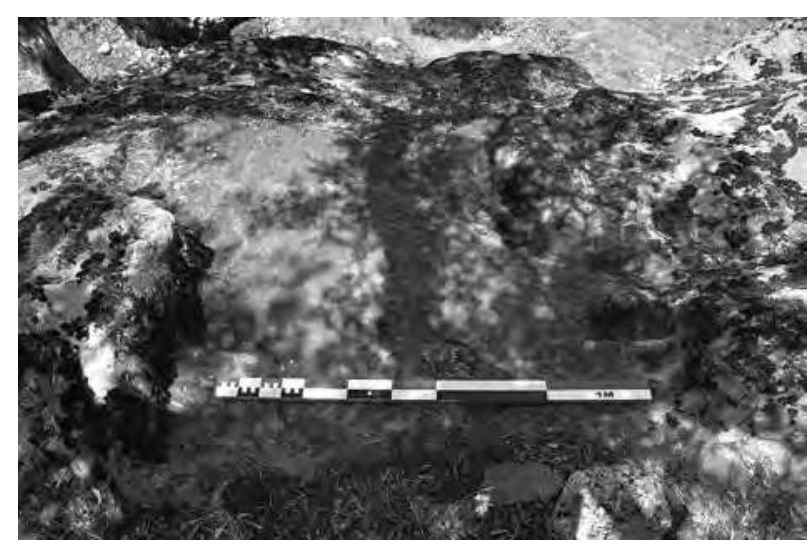

Fig. 2.4.73 : Le seuil taillé dans le rocher IKIZ005 (O. Henry).

(IKIZ001) est localisé sur une modeste plate-forme taillée (Fig. 2.4.74). La cuve est rectangulaire en plan, $1,86 \mathrm{~m}$ de long et $70 \mathrm{~cm}$ de large, et orientée Nord/Sud. Elle est partiellement remblayée par de petit blocs et, au moment de notre visite, inondée jusqu'à $54 \mathrm{~cm}$ sous l'ouverture. Un ressaut de $4 \mathrm{~cm}$ de large et $6 \mathrm{~cm}$ de profondeur court le long des côtés longs de la cuve, probablement pour recevoir des dalles de fermeture. La cuve est également encadrée au Nord et au Sud par un canal visant à drainer l'eau de pluie. Deux larges couvercles monolithes à double pente reposent sur le sol près de la cuve (Fig. 2.4.75). L'un d'eux, le plus grand, est encore partiellement posé en travers de la plate-forme portant la fosse, à l'Ouest (couvercle A), tandis que l'autre est renversé et repose à près de $2 \mathrm{~m}$ à l'Est (couvercle B) de la tombe. Le premier couvercle mesure $2,30 \mathrm{~m}$ de long, et $75 \mathrm{~cm}$ de hauteur maximale. Il a été sérieusement endommagé par des pilleurs et il lui manque près de la moitié de l'un des

Fig. 2.4.75 : Les couvercles A et $B$ de la tombe IKIZ001 (O. Henry).

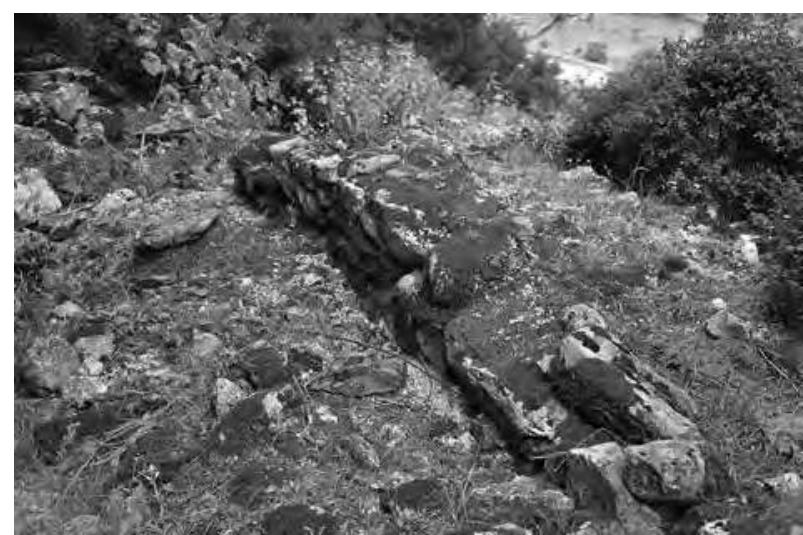

Fig. 2.4.72 : Le mur de fortification à İkiztaş (O. Henry).

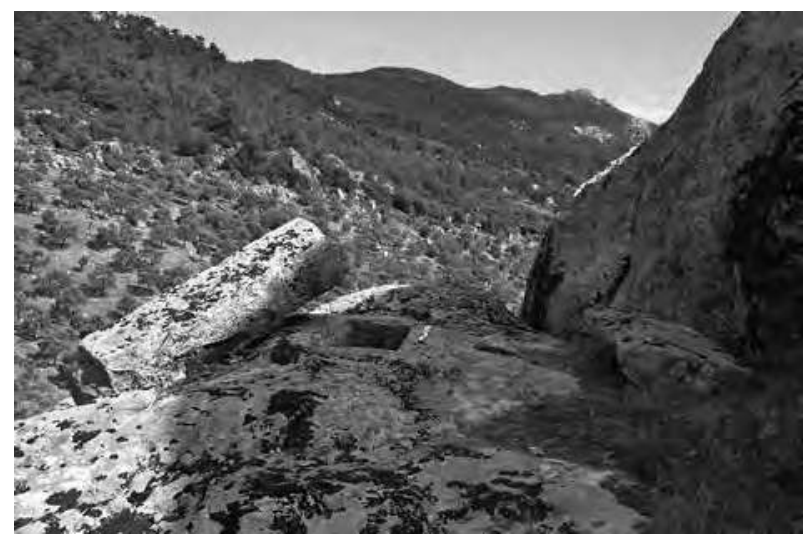

Fig. 2.4.74 : La tombe IKIZ001

(O. Henry).

IKIZOO

$\mathrm{OH}$
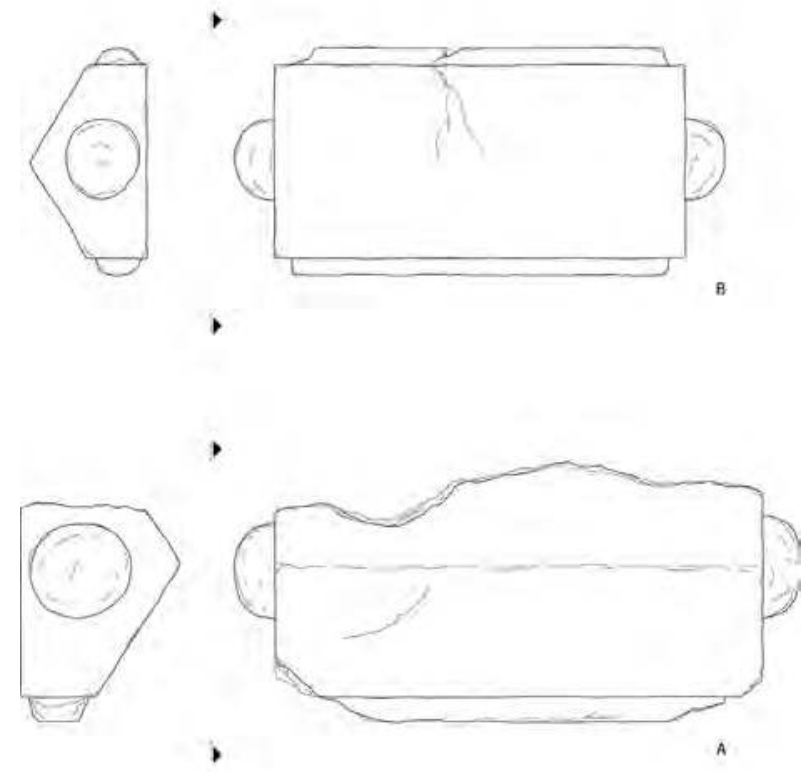
longs côtés. La largeur du couvercle, dans son état d'origine, doit avoir été d'environ 1,28 m. Le seul côté long préservé du couvercle montre un bandeau en saillie, de section carrée, presque aussi long que le côté du couvercle lui-même. Les deux petits côtés du couvercle portent chacun une projection, probablement des bosses de levage, de forme hémisphérique (de $44 \mathrm{~cm}$ de diamètre et $20 \mathrm{~cm}$ d'épaisseur). Le poids estiméducouvercledanssaformeintacteestsupérieurà 4,6 tonnes. Le deuxième couvercle (B) a une longueur de $1,94 \mathrm{~m}$, une largeur de $92 \mathrm{~cm}$ et une hauteur de $56 \mathrm{~cm}$ à la crête. Il semble être en parfait état (sa partie supérieure ne peut pas être entièrement observée du fait de la position renversée). Il montre le même agencement que le couvercle A de bosses / reliefs en saillie sur les côtés courts et longs. Le poids de ce couvercle, estimé à 2 tonnes, est nettement inférieur à celui du couvercle A. Nous avons cherché, sans succès, la présence d'une seconde tombe dans le voisinage immédiat, qui aurait pu justifier la présence simultanée de ces deux couvercles. Il semble donc que ces deux couvercles appartiennent à la même tombe et que, pour certaines raisons (probablement liées à la protection de la sépulture), le couvercle "léger" original a été remplacé par un autre, plus lourd.

La deuxième tombe (IKIZ003) est située à environ $25 \mathrm{~m}$ à l'Est de IKIZ001. Elle a été taillée dans une plate-forme aménagée sur le flanc est d'un imposant rocher (Fig. 2.4.76). Ladite plate-forme est cernée au Nord, au Sud et à l'Est par un canal taillé visant à drainer l'eau de pluie. La cuve a un plan rectangulaire et mesure $1,78 \mathrm{~m}$ de long et 70 $\mathrm{cm}$ de large. Elle est orientée Nord-Est/Sud-Ouest. Ses parois latérales montrent un profond ressaut de $8 \mathrm{~cm}$ de large, situé $28 \mathrm{~cm}$ sous l'ouverture. Les faces internes de la fosse sont, en outre, inclinées, offrant à cette dernière une section tronconique. La profondeur totale de la fosse ne peut pas être observée en raison de l'important remblai qui couvre ses parties basses. Néanmoins, on peut remarquer que le fond de la fosse semble avoir eu deux niveaux différents, marqués par un très large ressaut pratiqué dans la paroi nord. De tels agencements sont connus dans d'autres tombes rupestres et sont habituellement identifiés à des formes de coussin ou de tablette, en fonction de la hauteur du ressaut. Le couvercle de la tombe repose à l'Ouest de la cuve. Il semble avoir été grossièrement taillé et ne présente aucun angle droit net. Il est de plan rectangulaire et mesure $2,26 \mathrm{~m}$ de long et $1,20 \mathrm{~m}$ de large. Il offre une section arrondie avec une hauteur maximale de $40 \mathrm{~cm}$. Il ne porte aucun type de relief latéral tels qu'observés dans les autres tombes de la zone. On remarque, en outre, la présence de deux cavités taillées dans le flanc du tombeau sous la plate-forme. L'une est située à $20 \mathrm{~cm}$ au-dessous du côté Est de la fosse. Elle a la forme d'une mortaise circulaire, de $12 \mathrm{~cm}$ de diamètre et de $8 \mathrm{~cm}$ de profondeur. La deuxième cavité, située plus bas et plus au Nord, est plus grande. Son ouverture rectangulaire mesure $40 \mathrm{~cm}$ de haut et $30 \mathrm{~cm}$ de large. Elle est profonde de presque $50 \mathrm{~cm}$ et légèrement concave. Il est probable qu'il s'agissait là d'un réceptacle lié aux cérémonies funéraires (dépôt d'objets votifs, de nourriture, de lampe, etc.).

Entre les deux tombes, on remarque un escalier à six marches (IKIZ002) taillé directement sur le flanc du rocher portant IKIZ003 (Fig. 2.4.77). La destination de cet aménagement n'est pas claire. L'escalier semble mener vers le sommet du rocher, relativement plat, où l'on note la présence de nombreuses cavités rondes, apparemment naturelles mais fortement érodées, qui auraient pu servir de contenant pour liquides (IKIZ008). Il est impossible de confirmer si les tombes, l'escalier et les "cupules" naturelles sont liées d'une manière ou d'une autre (libation?).

Plus au Sud, à une dizaine de mètres sous la base du pic sud, on trouve une autre tombe rupestre (IKIZ004). Aménagée sur une étroite plate-forme rocheuse, elle est fortement érodée et presque intégralement remblayée. La cuve rectangulaire est orientée Nord-Ouest/Sud-Est. Elle mesure 2,18 m de long et 1,20 $\mathrm{m}$ de large. Un ressaut a été taillé le long des quatre côtés de la fosse. Il mesure $20 \mathrm{~cm}$ de large sur le côté long et de 12 à $16 \mathrm{~cm}$ de large sur les petits côtés. La profondeur de ce ressaut depuis l'ouverture de la fosse varie de $46 \mathrm{~cm}$ à l'Est jusqu'à presque rien à l'Ouest, en fonction de la destruction avancée de la roche elle-même. La profondeur maximale de la fosse semble avoir été de $68 \mathrm{~cm}$. Le dispositif de fermeture de la tombe a disparu.

Un dernier vestige (funéraire ?) a été repéré au pied du grand sommet. Il est composé de trois dalles, soigneusement taillées, placées de chant et disposées en U (IKIZ009). Il pourrait s'agir des trois côtés d'une tombe à ciste, qui mesurerait 1,80 m de long et $60 \mathrm{~cm}$ de large.

Aucun matériel associé n'a pu être récolté en relation avec les tombes. Le type de tombe et les détails de leur fosse ainsi que de leur couvercle semblent indiquer une datation comprise entre l'hellénistique tardif et la période romaine.

\section{La zone d'habitat}

Un groupe de vestige est encore visible à environ $200 \mathrm{~m}$ au Sud-Ouest du pic sud, à proximité et à l'intérieur du cimetière moderne. 


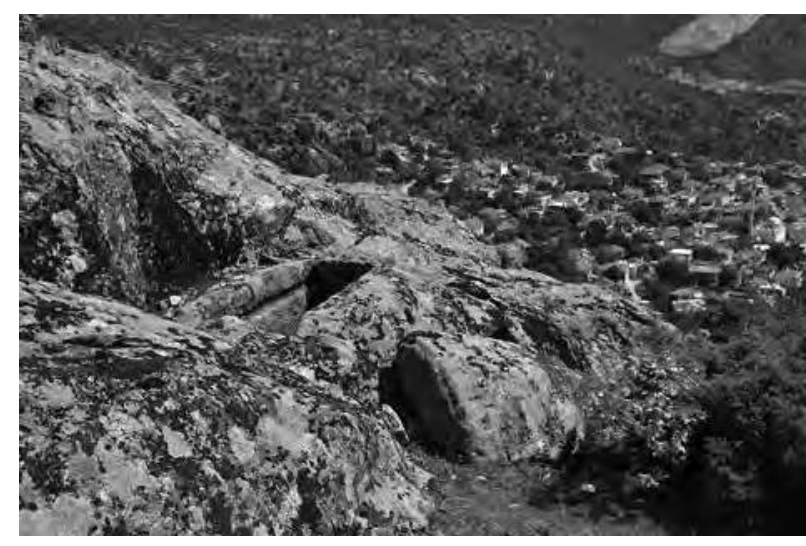

Fig. 2.4.76 : La tombe IKIZ003, depuis le Sud (O. Henry).

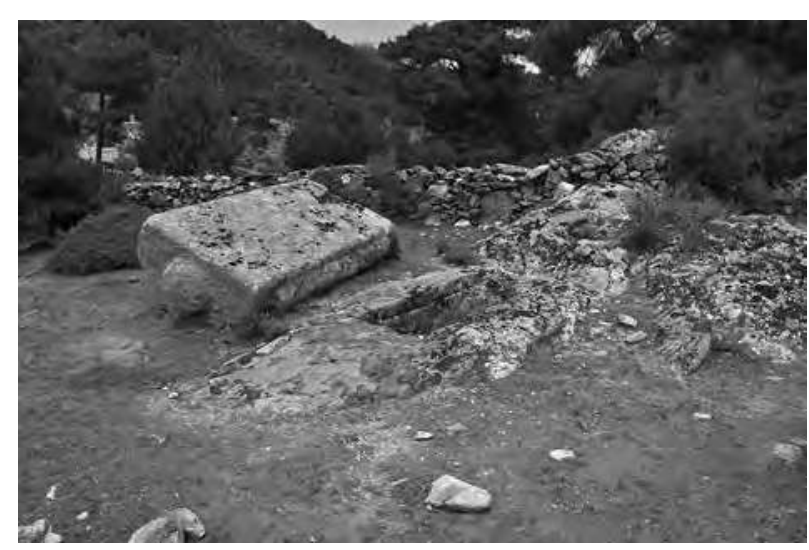

Fig. 2.4.78 : La tombe IKIZ010 (O. Henry).

Immédiatement à l'Est de la route on remarque un sarcophage rupestre taillé au sommet d'un modeste affleurement rocheux (IKIZ010) (Fig. 2.4.78). La cuve de la tombe est située au milieu d'une plate-forme étroite et allongée. Elle dispose d'un plan rectangulaire, orienté Nord-Ouest/Sud-Ouest, de $1,90 \mathrm{~m}$ de long sur $68 \mathrm{~cm}$ de large. Son fond est en partie remblayé de terre et de moellons jusqu'à $64 \mathrm{~cm} \mathrm{du}$ rebord supérieur. Les côtés longs de la fosse, ainsi que son côté court nord, montrent une ciselure de $10 \mathrm{~cm}$ de profondeur et $6 \mathrm{~cm}$ de largeur, probablement destinée à recevoir des dalles de fermeture. Un énorme couvercle à double pente $(\mathrm{A})$ repose, à l'envers, à moins de $1 \mathrm{~m}$ à l'Ouest de la cuve (Fig. 2.4.79). Il mesure $2,52 \mathrm{~m}$ de long, $1,26 \mathrm{~m}$ de large et $76 \mathrm{~cm}$ de haut à la crête. Il porte deux larges bosses de levage hémisphériques sur ses côtés courts, de 54 à $56 \mathrm{~cm}$ de diamètre, projetant sur 14 à $18 \mathrm{~cm}$. Chacun des longs côtés porte une moulure arrondie de $2 \mathrm{~m}$ de long, $30 \mathrm{~cm}$ de hauteur et projetant sur $6 \mathrm{~cm}$. Le poids du couvercle est estimé à plus de 5 tonnes. Un deuxième couvercle (B) se situe à $10 \mathrm{~m}$ à l'Est, plus bas dans la pente. Il a

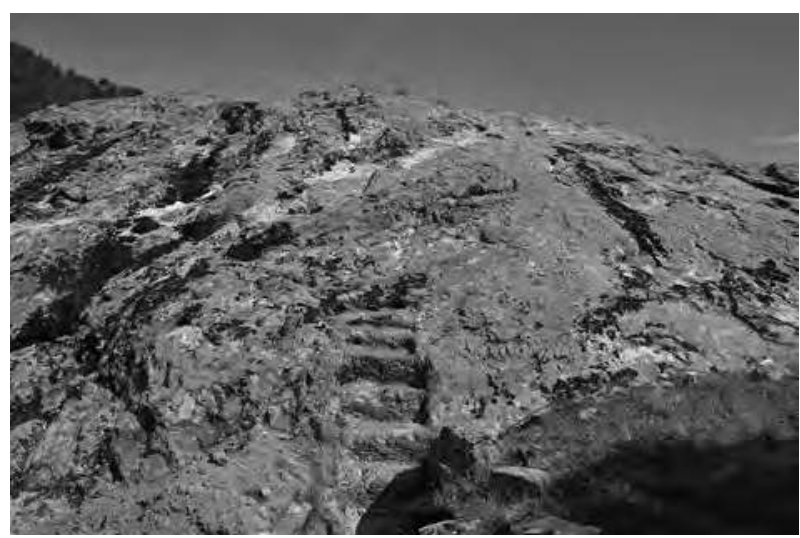

Fig. 2.4.77 : L'escalier rupestre (IKIZ002) taillé entre les tombes IKIZ001 et IKIZ003

(O. Henry).

IKIZOIO(2)

$\mathrm{OH}$

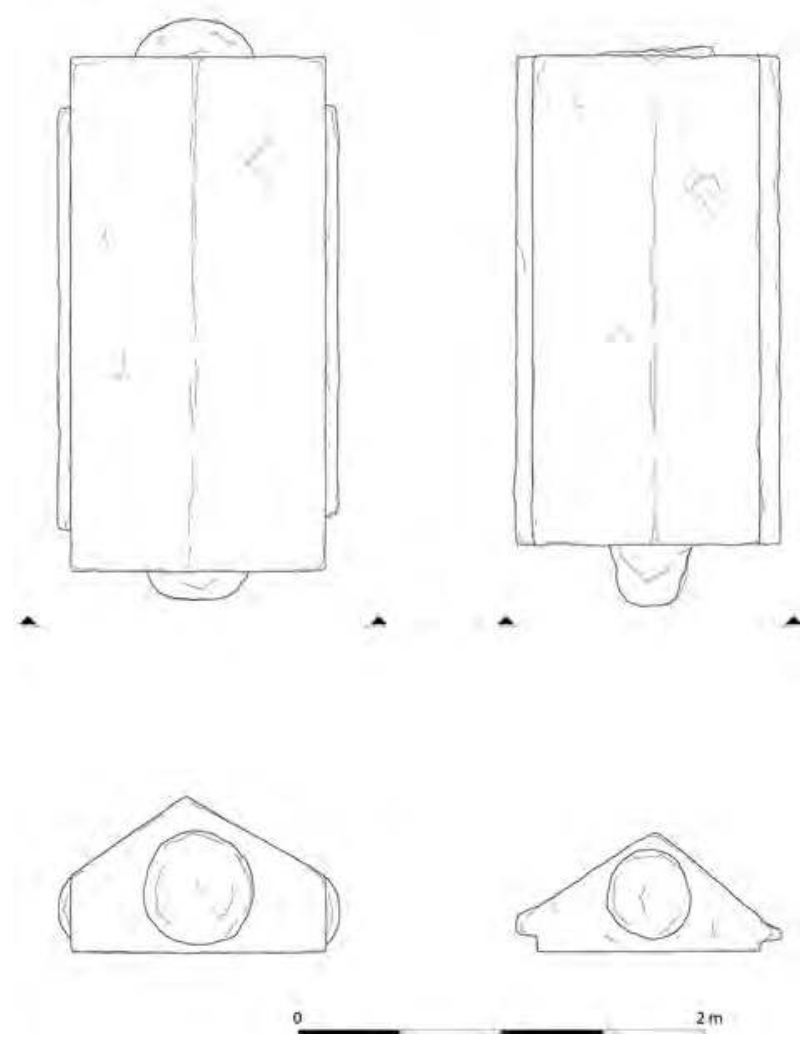

Fig. 2.4.79 : Les couvercles $A$ et $B$ associés à la tombe IKIZ010 (O. Henry).

les mêmes caractéristiques que le précédent mais a été taillé à plus petite échelle. Il mesure $2,40 \mathrm{~m}$ de long, $1,10 \mathrm{~m}$ de large et $58 \mathrm{~cm}$ de haut à la crête. Ses bosses en saillie sur les côtés courts et les moulures sur les côtés longs sont asymétriques, contrairement au couvercle A qui offre une symétrie presque parfaite. Les moulures des longs côtés sont aussi plus 


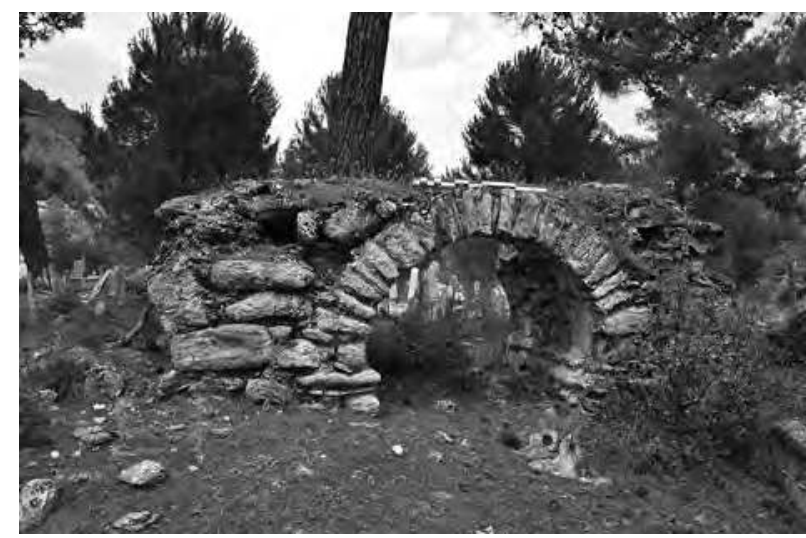

Fig. 2.4.80 : L'extrémité ouest de la structure IKIZ012, depuis l'Ouest(O. Henry).

fines (6 à $10 \mathrm{~cm}$ de hauteur) tandis que leur projection est davantage marquée (environ $10 \mathrm{~cm}$ ). Cette configuration à deux couvercles à proximité immédiate d'un tombeau unique rappelle clairement le cas de IKIZ001. Ici encore, et en l'absence d'une autre tombe à proximité, il est possible que le couvercle "léger" original ait été remplacé, par un système plus lourd, probablement afin d'accroître la protection de la tombe.

Posé sur le mur du cimetière, à 30 m à l'Ouest de la tombe, on repère un grand fragment de mosaïque (IKIZ011). Il mesure 64 sur $50 \mathrm{~cm}$ et $20 \mathrm{~cm}$ d'épaisseur. Il est composé d'un radier de $10 \mathrm{~cm}$, composé de petits moellons et d'un mortier blanc épais mélangé à du gravier. Celui-ci supporte un niveau de mortier blanc de $4 \mathrm{~cm}$ d'épaisseur qui comporte de petites inclusions de gravier. Au-dessus se trouve la mosaïque elle-même, composée d'un mortier hydraulique rosé, dans lequel on a inséré d'épaisses tesselles carrées de marbre blanc. Cette dernière couche mesure $6 \mathrm{~cm}$ d'épaisseur. La taille (env. $4 \mathrm{x}$ $3 \mathrm{~cm}$ ) et la manufacture des tesselles semblent indiquer une date de construction relativement tardive dans le courant de la période romaine.

A l'intérieur du cimetière moderne se trouvent les vestiges d'une imposante construction d'époque romaine (IKIZ012). Elle se compose d'un mur de 7,50 m de long et de 1,50 m d'épaisseur orienté Est/ Ouest. Le mur est construit à partir de blocs hétérogènes, liés par un mortier de chaux blanchâtre. Dans le liant du mur on remarque la présence, discrète, de quelques fragments de briques/tuiles. Dans sa partie occidentale le mur était percé de trois arches. Seule l'arche centrale est intégralement conservée (Fig. 2.4.80). La section orientale du mur est aveugle. Dans son parement sud ont a aménagé une niche de $90 \mathrm{~cm}$ de haut, $54 \mathrm{~cm}$ de large et $60 \mathrm{~cm}$ de profondeur. Les arches, d'une portée de 1,80 m,

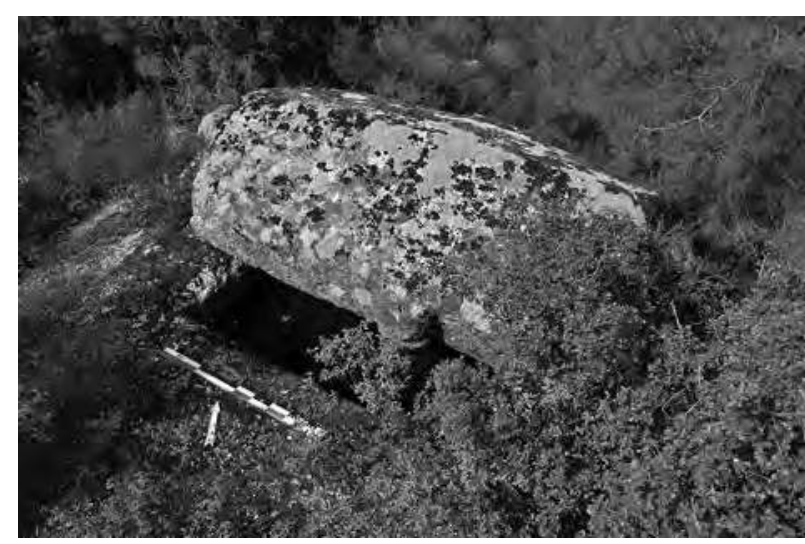

Fig. 2.4.81 : Le sarcophage rupestre IKIZ013, depuis le Sud (O. Henry).

reposent sur des piliers carrés, de 1,45 à $1,50 \mathrm{~m}$ de côté et séparées d'un espace de 1,55 m. On ignore la hauteur totale de ces piliers, remblayés sur leur quasi-totalité. L'arche conservée montre qu'elle n'est pas parfaitement circulaire, puisque son diamètre à la base est de 1,80 m alors que son rayon, calculé depuis le sommet du pilier, mesure 1,00 m. Des restes d'un enduit blanc peint de motifs géométriques rouges indiquent que le mur, ainsi que son intrados, étaient entièrement décorés, indiquant par ailleurs que cette construction faisait probablement partie d'une structure couverte.

Il est particulièrement difficile de définir, à ce stade de l'étude et dans de telles conditions de conservation, la fonction de ce bâtiment auquel appartient probablement la pièce de mosaïque trouvée à quelques mètres de là. Le bâtiment, qu'il s'agisse d'une maison ou d'un ensemble thermal (?), témoigne clairement de l'existence d'une riche occupation de la zone dans le courant de la période romaine.

Enfin, on repère un dernier sarcophage rupestre (IKIZ013) à $250 \mathrm{~m}$ à l'Ouest d'IKIZ012. Il est situé sur une grande plate-forme, taillée dans le flanc sud-ouest d'un modeste pic rocheux (Fig. 2.4.81). La roche autour de la tombe est très érodée et on ne remarque aucun aménagement supplémentaire sur la plate-forme (canal de drainage, moulure, etc.). La cuve elle-même est rectangulaire, orientée Nord-Ouest/Sud-Est. Elle a une longueur de 1,84 m et une largeur de $72 \mathrm{~cm}$. Elle était remplie d'eau et de pierres jusqu'à $30 \mathrm{~cm}$ sous l'ouverture lors de notre visite. Les parois latérales de la cuve ne montrent aucun ressaut tel qu'observé dans les autres sarcophages rupestres de la zone. Ils pourraient être cachés par le remplissage de la fosse. Les parois latérales de la cuve ont été taillées en contre-fruit, créant une section tronconique. La cuve est encore partiellement couverte 


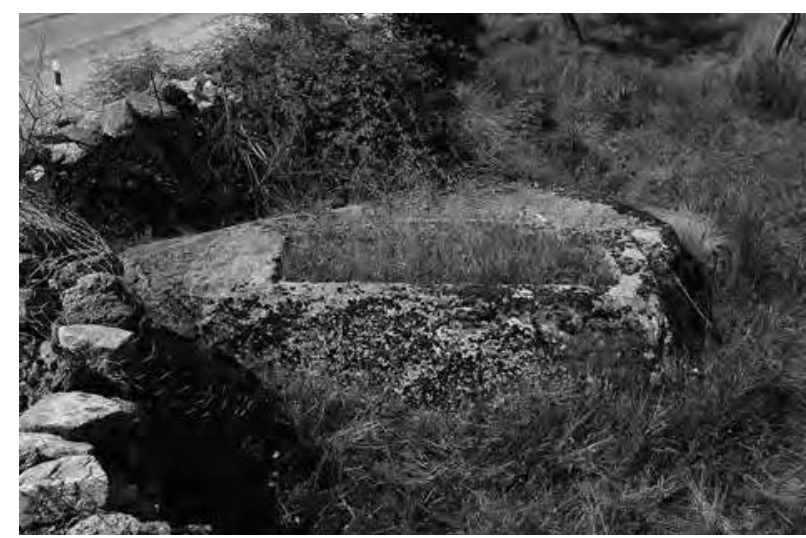

Fig. 2.4.82 : Le sarcophage rupestre IKIZ014, depuis le Nord (O. Henry).

par son couvercle massif dont le poids est estimé à 4 tonnes. Ce dernier mesure 2,40 m de long, 1,28 $\mathrm{m}$ de large et $56 \mathrm{~cm}$ de haut à la crête. Il ne dispose que d'un unique relief de bardage sur chacun de ses côtés. Ces "bosses" sont toutes de tailles différentes et leur disposition n'est pas symétrique. Aucun matériel associé à la tombe n'a pu être récolté. Néanmoins, les caractéristiques de la cuve à la section tronconique semblent indiquer une datation vers la toute fin de la période hellénistique ou dans le courant de la période romaine.

\section{B. Tekeoturağı Mevkii - Ferme ?}

A environ $750 \mathrm{~m}$ au Sud d'íkiztaş, nous avons visité un endroit qui avait été repéré par les autorités locales en 2010. A cette époque, un sarcophage rupestre et un broyeur avaient été enregistrés. Les deux structures ont été retrouvées cette année et documentées. Il s'est avéré par ailleurs que le broyeur appartient à un complexe bien mieux conservé que les documents officiels ne le laissaient supposer.

Le sarcophage rupestre (IKIZ014) est encore clairement visible, situé immédiatement derrière et à l'Ouest du muret qui longe la route moderne vers İkiztas (Fig. 2.4.82). La cuve du tombeau a été taillée au sommet d'une plate-forme étroite aménagée sur un affleurement rocheux peu élevé, à environ $1 \mathrm{~m}$ au-dessus du sol. La cuve est de plan rectangulaire, orienté Est/Ouest, de 2,10 m de long et $78 \mathrm{~cm}$ de large. Elle est presque intégralement remblayée et sa profondeur n'est visible que sur $15 \mathrm{~cm}$. L'ouverture de la cuve est entourée d'un bandeau en relief de $2 \mathrm{~cm}$ de haut et $23 \mathrm{~cm}$ de largeur. Le bord de la cuve dispose d'une ciselure de $8 \mathrm{~cm}$ de large et de $10 \mathrm{~cm}$ de profondeur, probablement destinée à recevoir des dalles de fermeture. Le couvercle de

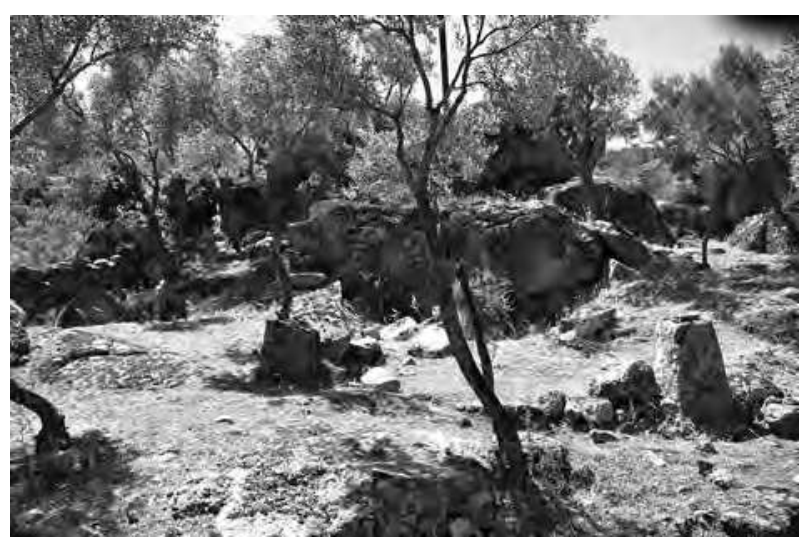

Fig. 2.4.83 : Le complexe IKIZ020, depuis l'Est (O. Henry).

la tombe a disparu. On remarque des marques d'extraction rocheuse sous le long côté nord de la fosse. Ces marques pourraient être liées soit à la taille de la plate-forme visant à recevoir la cuve, soit à une activité antérieure qui aurait offert aux concepteurs de la tombe un emplacement privilégié pour implanter la cuve.

Le complexe associé au broyeur est situé de l'autre côté de la route, à une trentaine de mètres de la tombe, à l'Est et au pied d'une grande formation rocheuse (IKIZ020). L'ensemble est exceptionnellement bien conservé puisque la presque intégralité des éléments constitutifs de l'huilerie est conservée (Fig. 2.4.83). Le pressoir était composé d'une pièce rectangulaire dont les murs sont conservés à certains endroits sur 1,50 m d'élévation. Les dimensions extérieures de cette pièce sont de 4,70 m d'Est en Ouest et de 5,70 m du Nord au Sud. La porte est excentrée, située à l'angle nord de sa façade est, où on note encore la présence in situ d'un des deux montants latéraux. A l'Ouest, le bâtiment s'appuie sur la paroi de l'imposante formation rocheuse dont le flanc ouest, qui forme l'une des quatre parois de la pièce, a été taillé à la verticale. Cette paroi porte la mortaise qui servait à recevoir l'extrémité du prelum, lequel devait être de large section compte tenu des dimensions importantes de la cavité $(55 \mathrm{~cm}$ de large, $60 \mathrm{~cm}$ de haut et $33 \mathrm{~cm}$ de profondeur). Autant que les remblais permettent de le constater, il semble que la mortaise se situe à $72 \mathrm{~cm}$ au-dessus du niveau du sol ancien, lequel est largement remblayé. Ce remblai empêche de confirmer la présence d'une meule ainsi que d'un contrepoids.

Le broyeur est situé à l'extérieur du pressoir, environ 2,60 m au Sud (à gauche sur la Fig. 2.4.83). Il a été renversé et est partiellement enfoui dans le sol mais semble être encore intact. Il est monolithique 


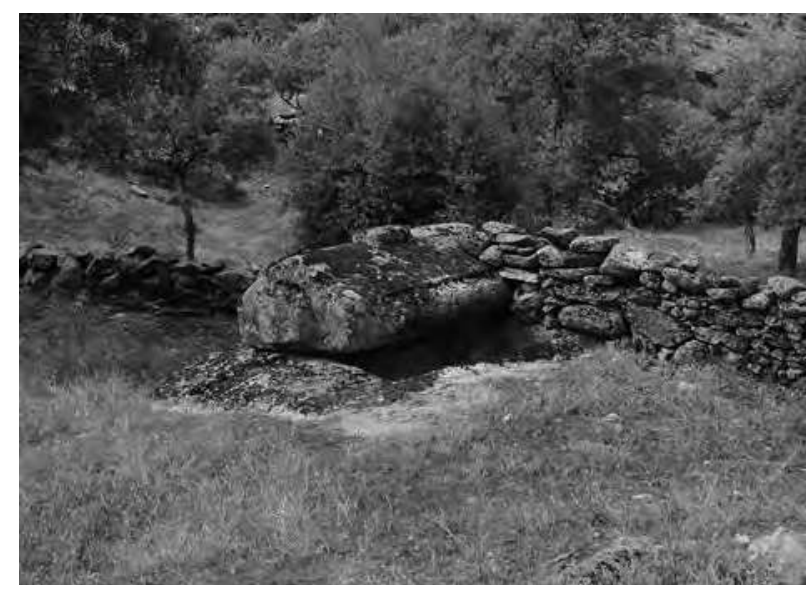

Fig. 2.4.84 : Le sarcophage rupestre IKIZ015, depuis le Nord (O. Henry).

et mesure près de $1,10 \mathrm{~m}$ de diamètre pour $60 \mathrm{~cm}$ d'épaisseur. Le rebord, d'environ $10 \mathrm{~cm}$ d'épaisseur, a été percé d'un côté par une rainure peu profonde (12 $\mathrm{cm}$ de largeur et $5 \mathrm{~cm}$ de profondeur). Le bassin mesure $95 \mathrm{~cm}$ de large et $28,5 \mathrm{~cm}$ de profondeur. Au fond, une cavité circulaire de $2 \mathrm{~cm}$ de profondeur et de $37 \mathrm{~cm}$ de diamètre, a été creusée afin de recevoir le milliarum. Au centre de cette cavité se trouve une mortaise carrée $(12 \mathrm{~cm}$ par $12 \mathrm{~cm}$ et $4 \mathrm{~cm}$ de profondeur) probablement impliquée dans le système de fixation du milliarum.

La présence simultanée du tombeau et du pressoir semble souligner l'existence d'un petit établissement dans la zone, peut-être une ferme, qui aurait appartenu au territoire lié à l'occupation repérée à İkiztaş. Peu de fragments de céramique et de tuiles ont été collectés sur place. Ils semblent toutefois homogènes et indiquent une période d'occupation autour du $1^{\text {er }}$ siècle ap. J.-C.

\section{Koca deresi - Habitat}

Le site de Koca deresi, localisé au sommet d'une colline, à 470-480 m d'altitude, avait déjà été repéré par les autorités locales en 2014, mais encore jamais documenté. Il est composé d'une tombe (IKIZ015), de vestiges d'habitations et de terrasses (IKIZ016).

Le tombeau est un sarcophage rupestre (IKIZ015) (Fig. 2.4.84). Il est situé au sommet d'un affleurement rocheux peu élevé. La cuve est orientée Nord/ Sud. Elle mesure 1,92 m de long sur $86 \mathrm{~cm}$ de large. Sa profondeur ne peut être observée que sur $72 \mathrm{~cm}$, du fait d'un important remblai. Une ciselure basse de $8 \mathrm{~cm}$ de large et $5 \mathrm{~cm}$ de profondeur a été taillée au sommet des parois est, ouest et nord de l'ouverture de la cuve. Celle-ci est également ceinte d'une bande saillante de $4 \mathrm{~cm}$ de haut et de 20 à $25 \mathrm{~cm}$ de largeur.
Le couvercle de la tombe est proche de sa position d'origine. Il a été légèrement poussé vers l'Est. Il mesure 2,40 m de long, $1,25 \mathrm{~m}$ de large et $77 \mathrm{~cm}$ de hauteur à la crête. Son poids est estimé à environ 4,8 tonnes. Il dispose d'un large relief sur chacun de ses petits côtés, tandis que ses longs côtés portent une moulure continue, presque aussi longue que le couvercle lui-même. Ces moulures ont des sections différentes : arrondie à l'Ouest, rectangulaire à l'Est. Une tablette rectangulaire, de $35 \mathrm{~cm}$ par $45 \mathrm{~cm}$, a été aménagée au sommet du couvercle, dépassant de 5 $\mathrm{cm}$ le faît de ce dernier. L'orientation de la tablette est légèrement désaxée par rapport à la crête du couvercle.

La pente de la colline au-dessus du tombeau semble avoir été entièrement occupée par des terrasses longues et étroites (IKIZ016), construites en moellons. Au sommet de la pente, sur une surface plane, on remarque de nombreux blocs quadrangulaires éparses, de grandes dimensions (plus de $1,50 \mathrm{~m}$ de long et $60 \mathrm{~cm}$ d'épaisseur), dont certains ont été percés d'une mortaise de fixation, soulignant le soin qui fut apporté, jadis, à la construction de cet habitat (?).

Très peu de fragments de céramique, principalement de pithoi, ont pu être collectés sur place. Les techniques de construction, ainsi que la tombe, semblent toutefois pointer vers une période comprise entre la fin de la période hellénistique et la fin de l'époque romaine.

\section{Bozuk Harım deresi Mevk. - Habitat/Ferme}

Plus haut dans le relief, à 250-300 m à l'EstSud-Est d'IKIZ015, et à une altitude de 620-640 $\mathrm{m}$, nous avons repéré un autre groupe de vestiges. Ceux-ci étaient inconnus jusqu'ici. L'ensemble est situé sur un terrain plat naturel dans la pente du Gözdengeç Sr., non loin de KETEN001. L'eau est omniprésente, ce qui a d'ailleurs motivé la construction d'une citerne moderne remplie d'eau fraîche, propre et potable. Malgré l'occupation moderne (certes légère), nous avons pu recueillir un peu de matériel céramique, principalement des tuiles et des fragments d'amphores. Ces derniers semblent tous appartenir à la fin de l'époque hellénistique / début de la période romaine.

Parmi les vestiges on note la présence d'un grand sarcophage rupestre (IKIZ017), aménagé au sommet d'un imposant rocher (Fig. 2.4.85). La cuve est orientée Nord/Sud. Elle mesure 2,02 m de long et $1,01 \mathrm{~m}$ de large. Sa profondeur ne peut être évaluée en raison de la grande quantité de remblais. Les parois nord, est et ouest de la cuve portent une ciselure de 5 à $10 \mathrm{~cm}$ de large et $22 \mathrm{~cm}$ de profondeur. 


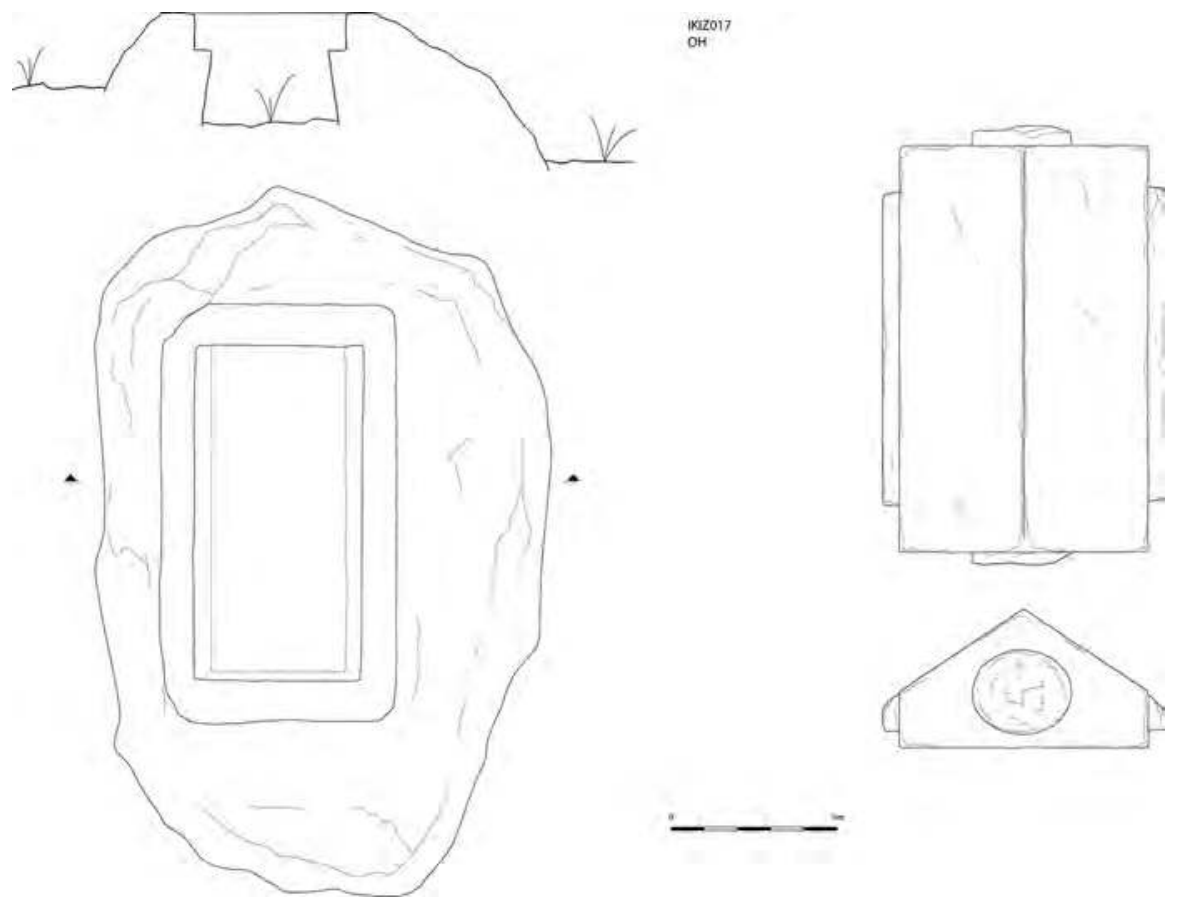

Fig. 2.4.85 : Le sarcophage IKIZ017 (O. Henry).

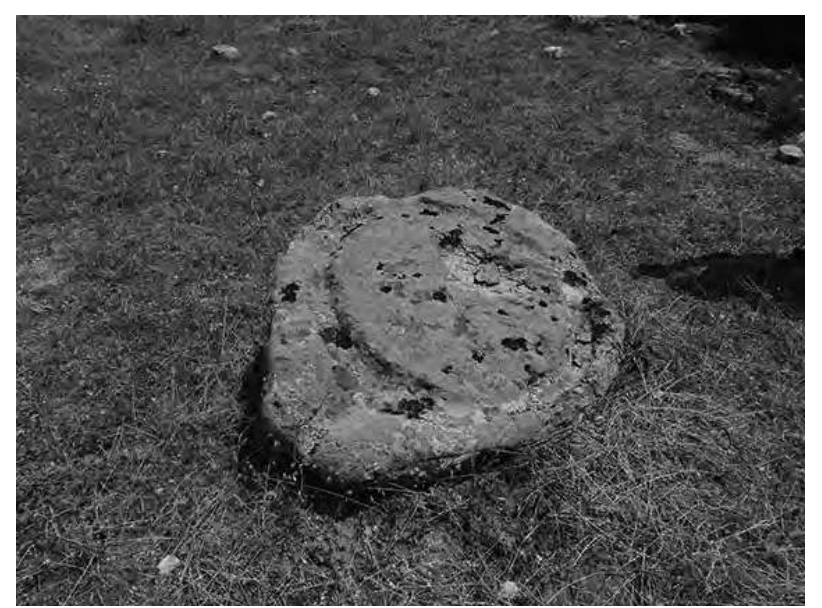

Fig. 2.4.86 : La presse IKIZ018 (O. Henry).

La cuve est également ceinte d'une bande en saillie, de $2 \mathrm{~cm}$ de haut et de 15 à $20 \mathrm{~cm}$ de large. Les parois latérales de la fosse ont été taillées en contre-fruit, créant ainsi une section tronconique. Le couvercle du tombeau est bien conservé, il a été poussé vers l'Est et intégré à l'intérieur d'un mur de parcelle moderne. Ce couvercle est massif, il mesure 2,45 $\mathrm{m}$ de long, 1,50 $\mathrm{m}$ de large et $84 \mathrm{~cm}$ de hauteur à la crête. Il dispose d'un relief hémisphérique (brisé) de $60 \mathrm{~cm}$ de diamètre sur ses petits côtés, et d'une longue moulure continue de 1,89 $\mathrm{m}$ de long, à la section rectangulaire $(22 \mathrm{~cm}$ de haut, projetant sur $10 \mathrm{~cm}$ ), sur ses longs côtés.
A environ $20-25 \mathrm{~m}$ au Nord-Est de la tombe, au-dessus d'une série de terrasses, on trouve une pierre de presse (IKIZ018). Celle-ci mesure 1,34 m de long et 1,08 $\mathrm{m}$ de large. La partie centrale est parfaitement circulaire, et mesure $75 \mathrm{~cm}$ de diamètre. Elle est ceinturée d'un canal de 5 à $7 \mathrm{~cm}$ de large (Fig. 2.4.86).

L'ensemble des éléments mis au jour ici (eau abondante, pressoir, tombe et matériel céramique) confirment la présence d'un petit établissement (une ferme ?) à l'époque hellénistique/romaine.

\subsection{CONCLUSION}

Les recherches de prospection menées autour du site de Labraunda durant cette saison 2017 ont démontré, une fois de plus, la richesse de la région, ainsi que sa profondeur historique, de la préhistoire à la période byzantine.

La prospection a été particulièrement réussie pour une première année, avec notamment la découverte de vestiges très importants, tant architecturaux que picturaux. La richesse et la variété de ces vestiges nous a surpris et il sera nécessaire de revenir sur de nombreuses zones afin de compléter la couverture de la région.

La répartition des sites découverts nous indiquent aussi que nous nous sommes limités cette année, sans vraiment nous en rendre compte, à une étroite bande de terre, situé à mi-chemin entre la plaine de Milas 
et les sommet du Latmos. Il sera donc nécessaire de tenter de couvrir ces zones-ci en 2018. De même, il sera nécessaire de revenir sur le site des peintures rupestres dont la documentation, particulièrement chronophage, n'a pas pu être terminée.

La mission 2018 sera donc étendue, par rapport à celle de 2017, avec trois semaines de pros- pection, dont une semaine entière dédiée à la zone ouest, tandis que les deux semaines restantes seront destinées à l'exploration de la région située à l'Est du site de Labraunda. Si les financements nous le permettent, nous prévoyons également de renforcer l'équipe qui comportera au total une dizaine de personnes.

\section{PROTECTION/CONSERVATION A LABRAUNDA}

\subsection{MARBLES (by E. Andersson)}

The marble conservation project in Labraunda was started by Agneta Freccero in 2010 and has been carried out yearly since then, making this the eight season. So far around 60 marble blocks have been treated, mainly focusing on inscriptions and important architectural fragments. This year we worked on fourteen blocks during a period of two weeks, from July $10^{\text {th }}$ until July $22^{\text {nd }}$. The elements that are included in the work are documentation, preventing biological growth, cleaning, consolidation and reparation of fractures. We also look for possibilities to move blocks to a better position, taking into consideration both original location and the preservation perspective.

This year the conservation team consisted of conservators Erika Andersson and Sona Holickova, archaeologist Görkem Çimen, archaeology students Merve Günal and Héloïse Frébault with assistance by archaeologists Mélanie Hauchard and Anıl Arslan.

During this year's season, we worked in several areas of the site: the Temple of Zeus and the Temple Terrace, the East Church, the East Propylon, the Oikoi and with some smaller blocks from the depot.

Prior to the other treatments, a biocide solution (Grönfri, 20 vol\% in water), is applied to the blocks to prevent further biological growth. Preferably, this should be carried out a year in advance to achieve maximum effect. Some of the blocks treated this year were not planned for in 2016, which meant the biocide treatment had to be applied just a few days before cleaning. Cleaning is carried out with spring water and natural pine oil soap (Grönsåpa) using soft brushes of different sizes and a scalpel for the removal of mosses and lichens.

Following cleaning, the marble surface is consolidated with diammonium phosphate (DAP), $50 \mathrm{gr} / 1 \mathrm{l}$ water with $10 \mathrm{vol} \%$ ethanol. This is to prevent sugaring of the marble surface (loss of the calcite crystals). This treatment has also proved to have a cleaning effect, which can be seen on previously conserved objects on the site. New research on the method suggests the addition of a small quantity of ethanol, $10 \mathrm{vol} \%$, in the DAP solution to improve the coating and reduce its porosity ${ }^{5}$. It was decided to try the method in Labraunda this year.

When necessary, fractures and fissures on the blocks are repaired with a mix of slaked lime and marble crystals of different sizes. The infills are retouched using aquarelle paint.

In 2017, the following blocks were conserved:

- Statue base with inscription inv. 112/P42 in front of the East Propylon (Fig. 3.1.1a-b)

- Sima placed on the brick altar, in the Oikoi (Fig. 3.1.2)

- Two architrave blocks with inscriptions, 114/ Y43 and Y 2011-1 in the East part of the Temple Terrace (Fig. 3.1.3a-b)

- Ionic capital inv. 3F-10 from the Temple Terrace broken in half, stored in the depot

- A small block located outside of the depot, possibly a fragment from an altar

- Architrave block inv. 35/D 99 in the Temple of Zeus

- Inscription inv. 104/P44 in the East Church

- Measuring block in the East Propylon (Fig. 3.1.4a-b)

The measuring block in the East Propylon was excavated by Swedish archaeologists in the 1950's. The block is broken in half and was mended at that time with concrete. The plan is to join the pieces together in the future. The old concrete mending was removed by dissolving it with water and ethanol, and then scraping it off carefully with a scalpel. The block was cleaned and consolidated this year.

Consolidation of three newly excavated blocks; an anta capital in the East Propylon and two blocks from the entablature of Andrôn A (Fig. 3.1.5), was done using the same method as above. Additionally, several fragments belonging to the architrave of the South Propylon have been cleaned and consolidated. Some of them are stored in the depot and others were found on the ground close to the architrave. A small 

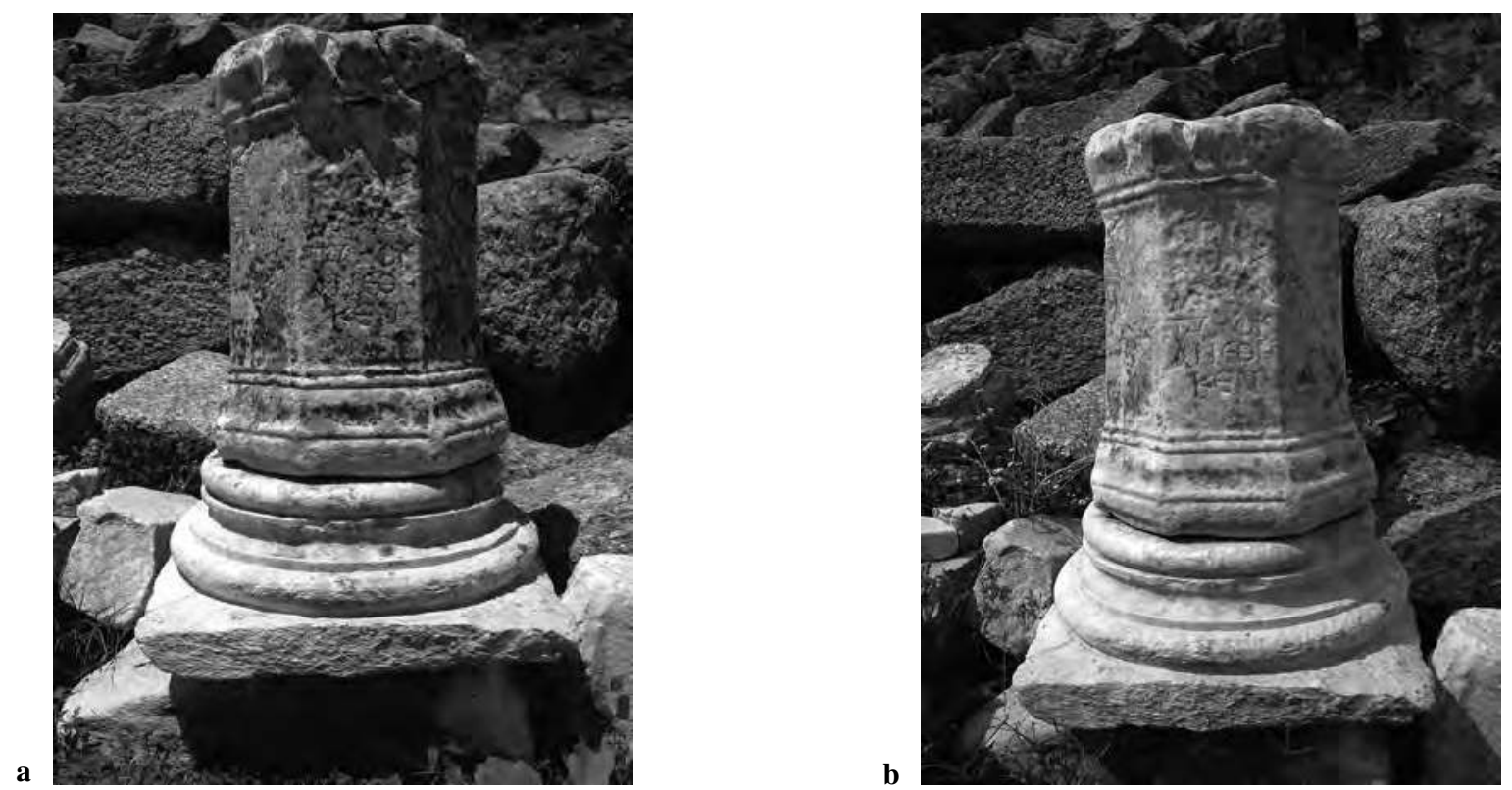

Fig. 3.1.1a-b : Statue base with inscription inv. 112/P42, before (a) and after (b) conservation (E. Andersson).

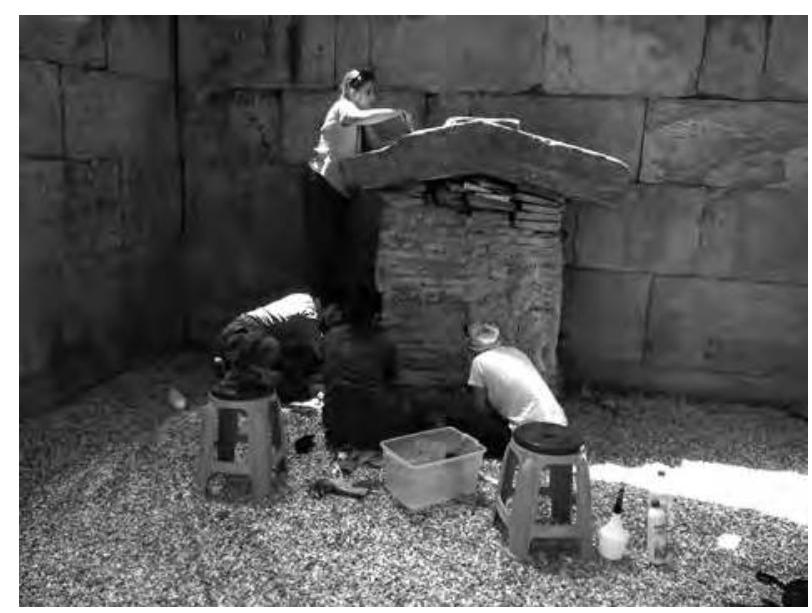

Fig. 3.1.2 : Work on the sima block placed in the Oikoi (O. Henry).
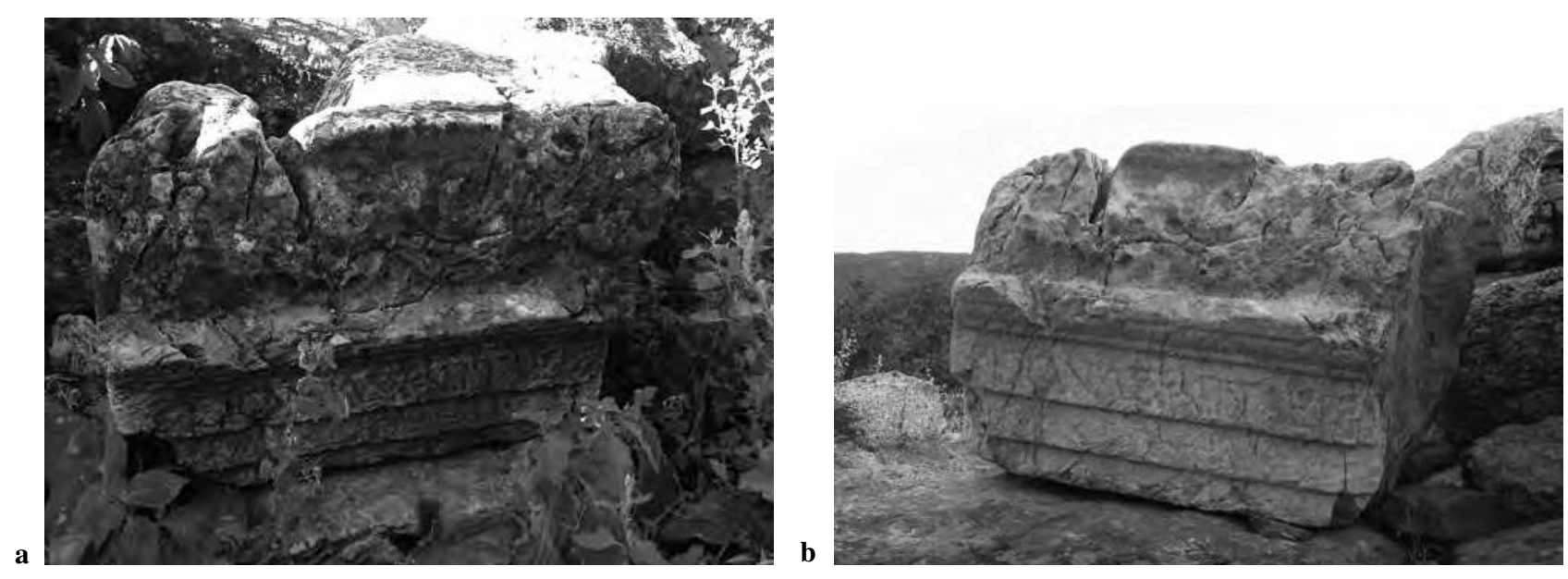

Fig. 3.1.3a-b : Inscription 114/Y43, before (a) and after (b) conservation (E. Andersson). 

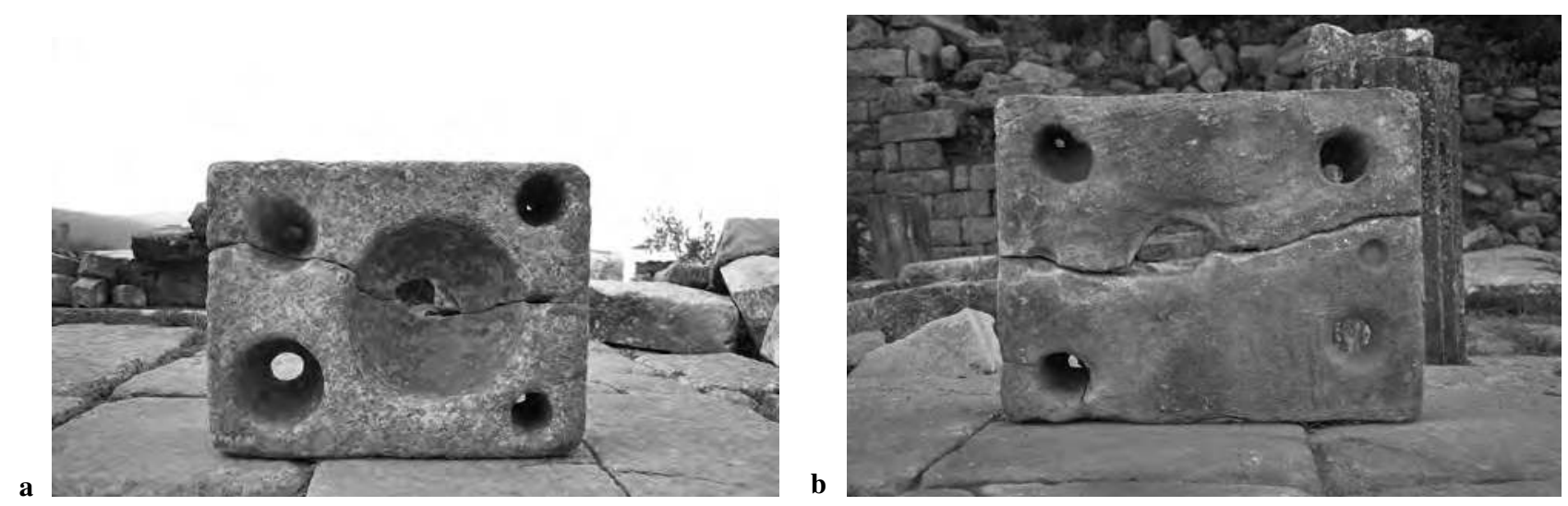

Fig. 3.1.4a-b : Measuring blocks, top (a) and bottom (b) faces, after conservation (E. Andersson).

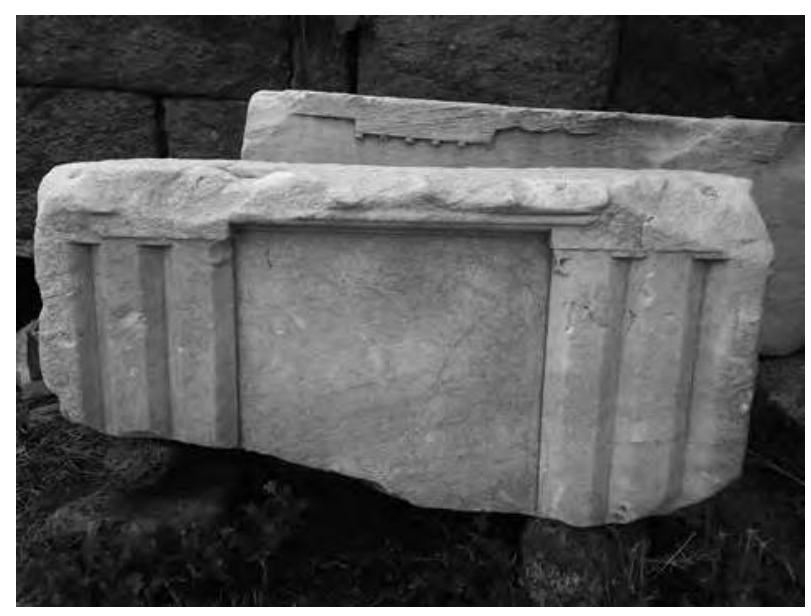

Fig. 3.1.5 : Blocks from the entablature of Andrôn A (E. Andersson).

marble block, possibly the fragment of a sphynx was found on the ground close to the site during our stay, and it was also cleaned and consolidated.

\subsection{DORIC HOUSE}

(by G. Çimen, M. Hauchart and Cr. Georgescu)

This part of the report regarding the activities at the Doric House at Labraunda is divided into three parts. The first part is a historical background, presenting the early excavations in the beginning of the 1950s. The second part presents the results of the new archaeological work which took place during the excavation season of 2017 and the third part covers the conservation and restoration work which was carried out in the same season, directly after the excavation.

\section{A. EARLY EXCAVATIONS OF THE P-BUILDING IN 1951 AND ITS EXCAVATION DIARY ${ }^{6}$} (by Görkem Çimen)

The 1950s designation of the P-building at Labraunda equals to what at present day is called the Doric House, situated adjacent with the East Bath on its western side. The P-building was excavated mainly in 1951, between July 9 and 26, under the supervision of the Swedish archaeologist Inge Dahlén (1920-2000). This excavation, as well as other excavated areas, was documented with drawings, photographs and a diary of 113 pages, written by Dahlén.

This part of the report is built on the contents of this excavation diary from 1951 and focuses on the excavation of the P-building as well as its immediate surroundings. Even though the diary also documents the work conducted around the area of the P-building, at the R-bath, which today refers to the East Bath and the East Church, this is excluded in the below text. The excavation of the P-building continued into the following excavation season of 1953 , but then mainly focused on documenting its foundation during a few days before moving eastwards, towards the R-bath. The 1953 work is also excluded from this report.

The 1951 and 1953 diaries have, however, never been published in their entirety. ${ }^{7}$ These two seasons have not resulted in any excavation reports either. Today this area is simply called the Doric House, but to be true to the old diaries the designation P-building is used in this part of the report.

6) This first part of the report stems from my bachelor thesis: The excavations of the P-building and the R-bath at Labraunda: Archaeology in the 1950s based on Inge Dahlén's three excavation diaries.

7) Jesper Blid has, however, made good use of these diaries in Labraunda 4, Blid 2016. 


\section{Uncovering the P-building}

Some photographs display the P-building before the excavation season of 1951. As they show, not much of the building was visible before the excavations, except for its west wall, the structure was totally covered with soil.

There are two main focus areas for the documentation of the P-building. The stylobate of the north wall of the building is one of them and the progress of its excavation can be followed day by day in Dahlén's notes and described as follows: The stylobate, with its mortises and traces of the columns, appears already in the afternoon of the excavation's first day (Fig. 3.2.1) ${ }^{8}$. The soil layer above it is estimated to be approximately $115 \mathrm{~cm}$ deep ${ }^{9}$. The direction of the stylobate is during the next day identified as WNW-ESE orientated ${ }^{10}$. The whole stylobate (Fig. 3.2.2) which is totally uncovered on the second day, had three columns between two antae. The middle block in the stylobate was displaced toward the building, which is also visible in the photographs (Fig. 3.2.1 and 3.2.2), and the whole north-eastern part of the building had sunk. Dahlén notes, however, that it could still not be determined if the divergent orientation could possibly depend on the sinking of the foundation ${ }^{11}$.

When the entire length of the stylobate was cleaned and photographed, a lot of preserved bronze cramps were identified both at the topmost course of the foundation and at the stylobate ${ }^{12}$. The middle block of the stylobate was later slightly raised and fitted in so that it was placed on the same line as the other blocks on both sides (Fig. 3.2.3 and 3.2.4). The block situated westwards has a one decimeter wide open conduit (Fig. 3.2.2, 3.2.4 and 3.2.5). Dahlén notes a crack along it that goes through the foundation ${ }^{13}$. The final P.S note on the last page of the diary, which is in fact dated to 1953 , includes a causal explanation of his archaeological thinking about it: "The open conduit in the middle of the stylobate is suitable for the small pipe of the tepidarium" ${ }^{14}$, which was excavated in 1953 . This open conduit in one of the stylobate blocks (Fig. 3.2.2, 3.2.4 and 3.2.5) corresponds to a vertical conduit in the southern part of the western outer wall where the foundation had cracked and burst open ${ }^{15}$. This description was directly followed by its sketch in the diary. Dahlén explains the next day that archaeologist Kristian Jeppesen, who was a member of the team, went under the floor in the southern part of the west wall where the floor was identified to be much thinner than in the south-eastern corner. It appears it had sunk strongly and was therefore filled with layers of stone, plaster and tiles ${ }^{16}$.

Four column drums of marble (P22-25) with and without cutting for the beam ends were also found overthrown immediately in front of the building, which were later placed on the stylobate (Fig. 3.2.1, 3.2.3 and 3.2.4) ${ }^{17}$. The block in yellowish stone (P31) is mentioned to be laid by them in the northernmost part of the west wall (Fig. 3.2.3 and 3.2.5) ${ }^{18}$. Dahlén distinguishes in his notes the architectural elements which were not found in-situ and explains explicitly what kind of relocations that had been carried out by them.

The second focus area in the diary is the multiple floor layers of the P-building. Their continuous archaeological work can be presented as follows, based on Dahlén's own descriptions: The floor of the building was found when the south-western quarter was unearthed. A "wall" going across the structure is identified and the south part of it is recorded to have a mosaic floor of 3-4 cm large "stones", as partly seen in the lower part of Fig. 3.2.5. The identification of "wall" is most probably referred to the foundation of the parapet (Fig. 3.2.5), consisting of marble blocks, which separates the building's north area from the south. The red plaster leaning up the edges are noted to resemble a bathroom floor. Directly after these descriptions several water pipes running from the north and the north-west of the building are also mentioned ${ }^{19}$. The above-mentioned marble mosaic is temporarily exposed only in the south-western quarter of the floor but can be traced in several places.

8) Dahlén 1951: 6.

9) The soil layer in the north of the P-building is additionally documented to be approximately $1.60-1.65 \mathrm{~cm}$ above the stylobate level in Dahlén 1951: 42.

10) Dahlén 1951: 7.

11) Dahlén 1951: 9-10.

12) Dahlén 1951: 11.

13) Dahlén 1951: 12.

14) Dahlén 1951: [113].

15) Dahlén 1951: 43-44.

16) Dahlén 1951: 49.

17) Dahlén 1951: 13, 30.

18) Dahlén 1951: 13, 31.

19) Dahlén 1951: 22-23. 


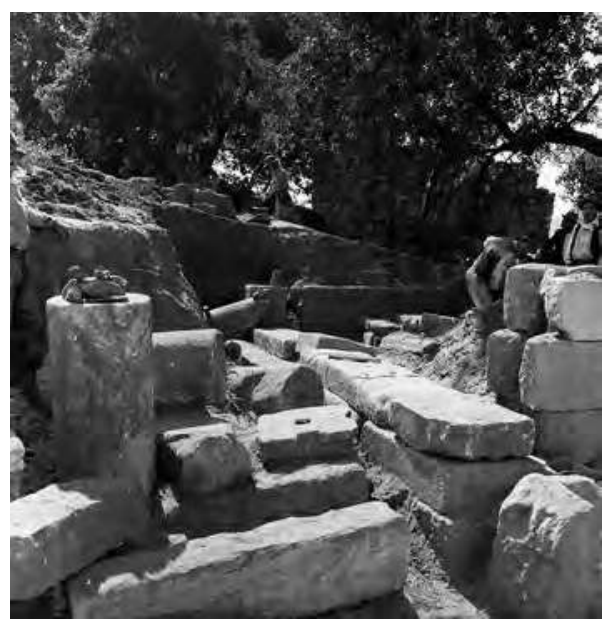

Fig. 3.2.1 : Stylobate of the P-building, seen from NW, also showing the column drums in front of the building and the level of the soil covering the area (from Labraunda archive).

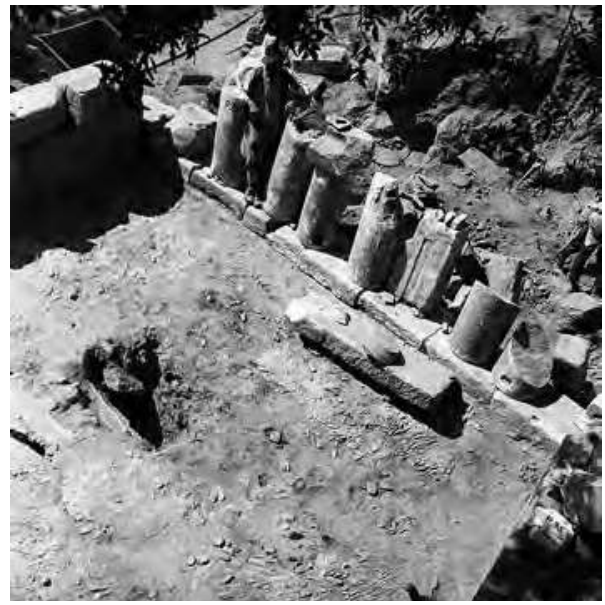

Fig. 3.2.3 : Northern area of the P-building, seen from $\mathrm{SE}$, with the visible stylobate and the architectural elements of marble (from Labraunda archive).

The floor layers in the south-eastern quarter (Fig. 3.2.6) consist of the following explanations: The topmost is a floor level of small tile pieces in plaster, particularly well-preserved in the south-eastern corner where the tile pieces with an average length of about $7 \mathrm{~cm}$ are located in quite well-arranged rows ${ }^{20}$. This description is followed by a simple sketch in the diary, showing the floor of the south-eastern corner with the tile pieces in situ. Approximately $7 \mathrm{~cm}$ below the tiles and the pink mortar, there is an older floor, consisting of a marble mosaic of simple type in similar mortar. These mar-

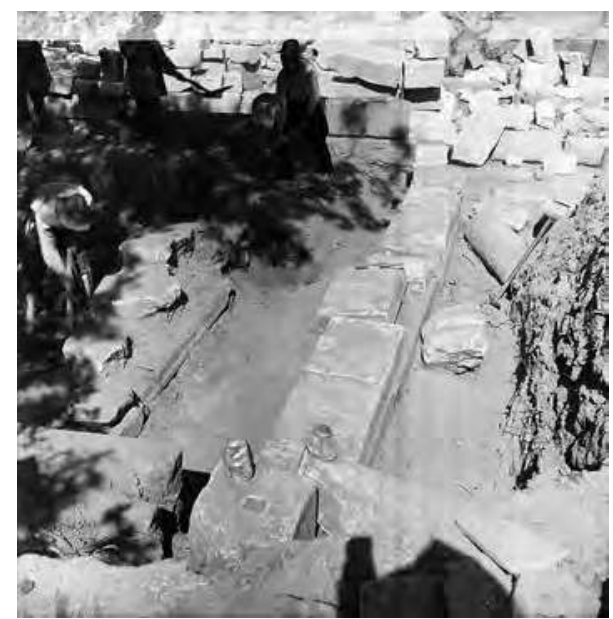

Fig. 3.2.2 : Stylobate of the P-building, seen from ENE (from Labraunda archive).

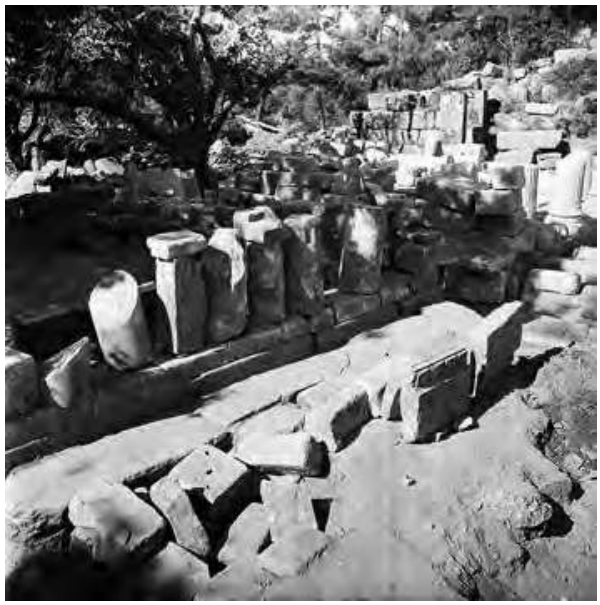

Fig. 3.2.4 : P-building, seen from NE (from Labraunda archive).

ble tesserae are about $3 \mathrm{~cm}$ large. Below the mortar there is a layer of elongated stones. Thereunder there is mortar again and in many places, there is a void space below. In one place the tape measure goes down a few meters ${ }^{21}$. The location of this "one place" is, however, not explained clearly. As these explanations of the archaeological work depict, the stratigraphical descriptions of the floor with the different layers of tile pieces, "marble mosaic" and stones are textually well-documented in the diary.

Amongst the notes, there is an emphasis on the strong sinking of the foundation where Dahlén tries

20) Dahlén 1951: 39.

21) Dahlén 1951: 39-40. 


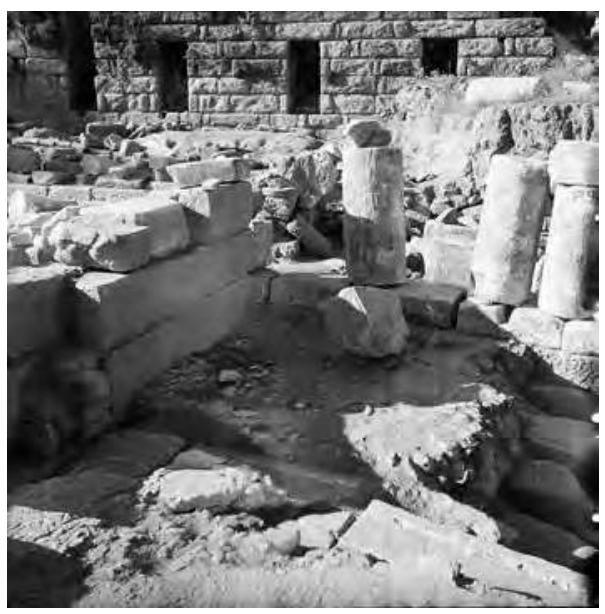

Fig. 3.2.5 : North-western quarter of the P-building, seen from SE, also showing the open conduit in the stylobate below the middle column drum, parapet in the middle and the marble mosaic floor of the south-western quarter (from Labraunda archive).

to explain for instance the changes of the floor. The tile floor in the south-western corner is described to be over the marble mosaic, but at a specific spot the marble mosaic appears to be above the tile floor. Dahlén concludes therefore that it is an indication showing that the floor was hastily patched up after a strong sinking of the foundation ${ }^{22}$. The cavities in the building, as noted, may also depend on a very large sinking of the foundation. He additionally explains that the foundation and floor have been separated and large cracks have been formed, but they have not been filled, perhaps because the floor has prevented soil from flowing down ${ }^{23}$.

Most of "the marble wall" that went across the building in an east-west direction was uncovered by Jeppesen after one week of work ${ }^{24}$. Dahlén's following descriptions on the architectural details of it refer to the parapet (Fig. 3.2.5 and 3.2.7): The floor blocks, across the floor, are five in total, of which the outermost are half the size of the others. They rest on a stone wall. The joints appear to originally have been carved with refined technology. There is a string of plaster (the strong white) in two lines which hide two carved grooves, the southern one is well-made with a vertical groove and the northern is only a carved groove. The plaster is in one place shaped as if there had been a block (tile) with a nar-

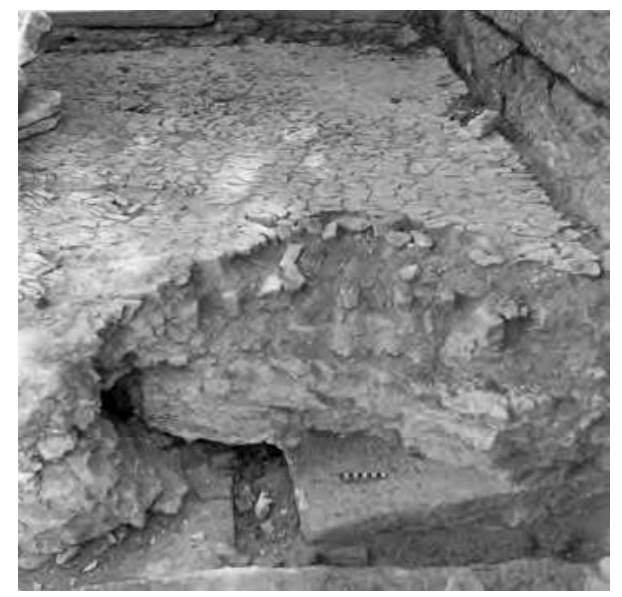

Fig. 3.2.6 : Floor in the south-eastern quarter of the P-building, seen from $W$

(from Labraunda archive).

row groove in $\mathrm{it}^{25}$. These descriptions are followed by two sketches, one showing the joining part of two marble blocks with the carved grooves filled with plaster (Fig. 3.2.7) and one depicting a marble block from profile.

\section{Documentation methods}

Both the archaeological work, where Dahlén explains what is being unearthed, and the archaeological finds, discovered in the area during the excavations of the building, are described thoroughly in his notes. All these descriptions give the reader a visual understanding of the 1951 excavation.

The find-spots of the architectural elements and archaeological finds are most commonly described as clearly as possible in the notes, by placing the finds in relation to the distances of visible structures or blocks, used as reference points. An example of this is: "P16. A column base was found somewhat below the stylobate level, $180 \mathrm{~cm}$ north of the stylobate, in front of the first intercolumn from the west (= west of the middle column)"26.

Marble. The documented archaeological finds in the 1951 diary consist almost only of architectural elements of marble, specifically marble blocks. Except for only a few blocks, the descriptions of

22) Dahlén 1951: 43.

23) Dahlén 1951: 59.

24) Dahlén 1951: 49.

25) Dahlén 1951: 56-57.

26) Dahlén 1951: 25. 


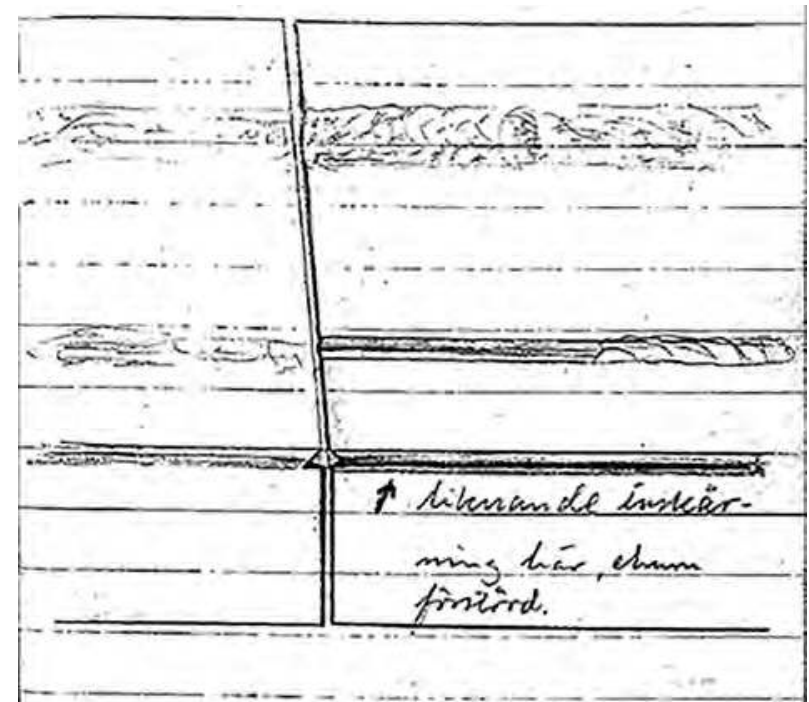

Fig. 3.2.7 : Parapet

(I. Dahlén in Dahlén 1951 : 57).

the architectural elements are, however, in most cases not accompanied by sketches or drawings in the diary. Some examples of the documented marble elements are metopes, triglyphs, column drums, capitals and marble moldings. Every documented architectural marble element is designated with the letter P, referring to the P-building, and given consecutive block number.

There is, however, one challenge concerning the documentation of the finds, specifically marble elements. As the excavation started in the P-building and then moved eastwards towards the R-area, the spatial context had changed. Nevertheless, Dahlén did not separate the documentation of the finds in the diary, but continued to refer to the marble blocks with the "P" designation. Even though an architectural element was found in or closer to the R-bath area, Dahlén continued to assign it a P-number. This means that, following the daily work which already is a challenge by itself as it requires systematic close reading, the choice to use $\mathrm{P}$ for all architectural elements adds to the complexity of analyzing the diary and contextualizing the find-spots.

Inscriptions. The letters of the inscriptions are always noted in Greek and are not translated to Swedish by Dahlén. The state of preservation of the blocks and the condition of their surfaces are most often explained. The measurements of the inscribed blocks and lengths of the inscriptions with the heights of the letters are thoroughly record-

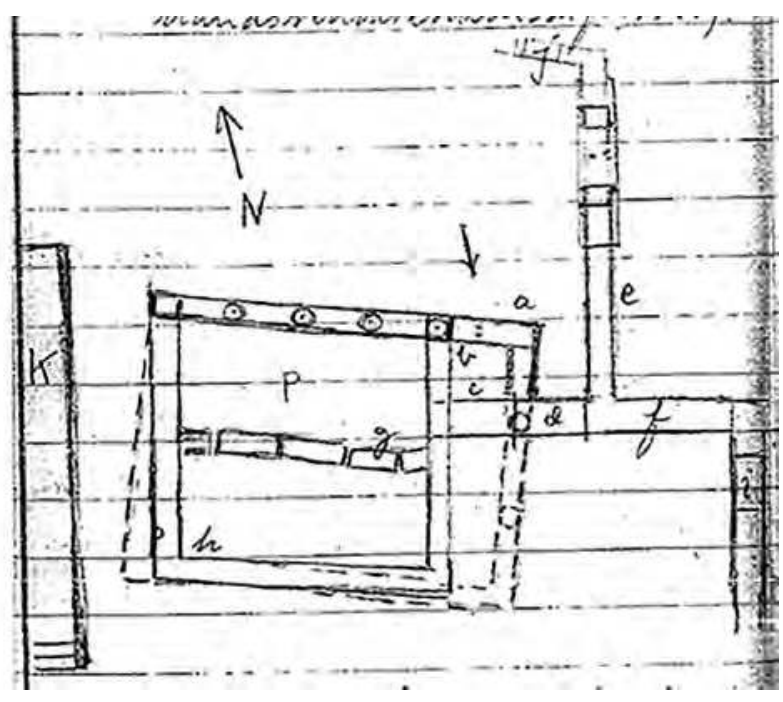

Fig. 3.2.8 : "Reconstructed" plan of the P-building (I. Dahlén in Dahlén 1951 : 80).

ed. According to the documented records in the 1951 diary, six blocks with inscriptions nearby the P-building were found. One of them, identified as $\mathrm{P}$ $12^{27}$ is an architrave block of marble with a groove underneath and is found $1.5 \mathrm{~m}$ north of the building, slightly west of its centerline. This block consists of six Greek letters: O M N $\Omega$ M Y. At the end of the page in the diary, the dedication of the P-building has been chosen by setting the Hecatomnids in order as: Maussollos, Idrieus, Pixodaros (with a question mark). Maussollos and Pixodaros have, however, been crossed out and Idrieus has not, but the motive is not given in the notes ${ }^{28}$.

Small finds. The number of the documented small finds in the 1951 diary are limited, especially in comparison to marble. This limited amount of the small finds represents in most cases pottery sherds which are documented with textual descriptions and are mostly complemented with sketches but do not receive any find number.

Coins. Five bronze coins are documented in the 1951 diary, but none of them were found in the P-building or in its immediate surroundings. The indications of the find-spot heights in this area are, however, most of the times related to the P-building, using the descriptions as "surface", "the stylobate level", "above the floor" and "above the pavement". An example of this is: "found by P 52, $30 \mathrm{~cm}$ under the stylobate level" 29 . Descriptions and comments on visible letters and motives of both sides of the

27) Dahlén 1951: 20-21. It was later placed on the column drum $P 25$ on the stylobate.

28) It is the inscription (I.Lab 20), which suggests that the P-building is dated to Idrieus ruling years. See Labraunda III:2, 17f. A conservation treatment was carried out on this marble inscription in 2016 and today it is placed on the platform which was constructed in 2017 north of the P-building.

29) Dahlén 1951: 69. P52 is the given marble block number and this block is situated in the area of R. 
coins are well-explained. There is also an explicit tendency to date the coins as well as give references to their location.

\section{Dahlén's interpretations on the architectural development of the P-building}

Dahlén not only documents the archaeological work factually in the 1951 diary but often adds his own understanding and interpretation regarding the P-building ${ }^{30}$ as well as the function and use of the finds. The first sketch showing a simple plan of the P-building is dated to July $14^{31}$. The displaced shape of the stylobate with the cramps for the four columns are shown on the plan. The find-spot of the three newly found blocks, of which one is a tympanon block of stone, are also situated on the sketch.

A more developed sketch showing the plan of the P-building is dated to July 20 (Fig. 3.2.8) and explained by Dahlén as "the P-building's relation to walls in the east and first-hand reconstruction attempt" 32 . This "reconstructed" plan is organised by giving letters from "a" to " $\mathrm{j}$ " for specific areas to present related archaeological data. This sketch together with its explanations shows numerous significant details on his understanding of the structure. These are: the irregular orientation of the building, the columns situated on the displaced stylobate, the original building phase with its displaced current walls and the stereobate "a" which continues outside of the current building. The foundation is recorded to continue into the "d-f wall". " $d$ " is identified as a door that is covered-up and "e" is defined as "bad quality, late wall". "g" is defined to have been displaced about half a meter due to the sinking of the foundation. The wall by the corner of " $h$ " is described to be significantly displaced southwards ${ }^{33}$.

All these identifications and interpretations are followed by a P.S. note, written one week later, on July 27. According to the note, Dahlén had three types of evidence of an older building that preceded the P-building. The first one is that it contains "well-cut, yellow re-used stone (gneiss)", the second one is "the stereobate angle in the northeast" and the third one states that "a sharp angle by the north-western corner that should mean that the west wall has been repositioned to give passage" ${ }^{34}$.

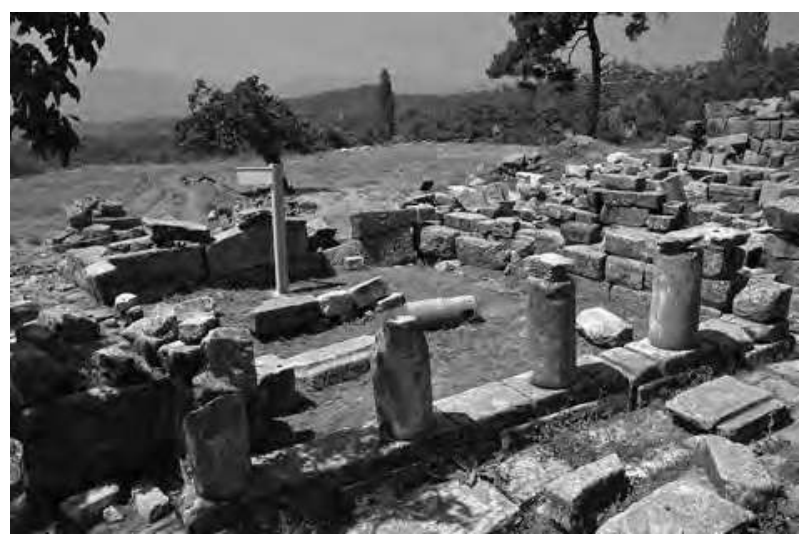

Fig. 3.2.9 : Vue d'ensemble de la Doric House avant le début des travaux (M. Hauchart).

\section{Conclusion}

Inge Dahlén's handwritten excavation diary from 1951 is a set of texts that contain explanations of the archaeological work conducted at the P-building together with practiced documentation methods and archaeological discoveries. There are challenges and absences in the notes which in some cases complicate the archaeological considerations. Dahlén's diary, however, provides indications and explanations for our archaeological understanding of the past work that has already been done and how it has been conducted as well as how the archaeological data has been interpreted. The diary contains numerous examples where Dahlén interprets archaeological data and finds and presents his own analysis of their possible functional use. The diary is written in a very visual and descriptive way, includes very well depicted drawings and makes for an important contribution of the past for the present-day research. For the current and future excavations that are being conducted in the same area at Labraunda, especially the East Bath and the Doric House, it is useful and worthwhile to further analyse Dahlén's explanations, reflections and interpretations on the P-building.

\section{B. L'INTERVENTION DE 2017} (par M. Hauchart)

Les travaux dans le bâtiment de la "Doric House" ont commencé le 21 juillet 2017. Le but était

30) The P-building was later suggested by Westholm to function as a "treasure house"; see Westholm 1963: 98. Both Hellström and Blid suggest on the other hand, that the P-building functioned as a "fountain house"; see Hellström 2007: 74 and Blid $2016: 127$.

31) Dahlén 1951: 20.

32) Dahlén 1951: 80.

33) Dahlén 1951: 81-83.

34) Dahlén 1951: 83. 
de remettre au jour les niveaux de sols, découverts puis remblayés lors des fouilles suédoises des années 1950. Le secteur était occupé par divers blocs de marbre posés sur les remblais. La première partie du travail a consisté à les déplacer à l'extérieur du bâtiment. L'épaisseur moyenne des remblais était inférieure à $40 \mathrm{~cm}$, ce qui a permis de les évacuer rapidement pour retrouver tous les niveaux de sols et vider les sondages profonds (Fig. 3.2.9).

Le nettoyage a offert l'opportunité de faire de nouvelles observations et de compléter les données sur l'organisation de l'espace et des sols.

\section{Description}

L'espace est divisé en deux par une rangée centrale de blocs de marbre orientée Est/Ouest et qui se prolonge vers le Sud, le long du mur ouest. Cet aménagement définit un secteur dans la moitié sud du bâtiment où sont concentrés les niveaux de sol.

Ces blocs de pourtour présentent sur leur face d'attente des rainures comblées de mortier blanc. Le profil de ces rainures diffère : sur les blocs alignés Est-Ouest, il s'agit de deux rainures parallèles : la rainure sud dispose d'une section en $\mathrm{V}$ de 3 à $4 \mathrm{~cm}$ de large, alors que la rainure nord est moins profonde, ses contours sont moins nets et elle est large de 4 à $5 \mathrm{~cm}$. Sur le retour ouest, seule la rainure sud perdure, en formant un coude sur le bloc d'angle (nord-ouest) alors que la rainure nord prend fin à l'extrémité dudit bloc.

Le bloc sud-ouest, manquant à la fouille, a été retrouvé parmi l'ensemble des blocs qui avaient été déposés sur les remblais. Il a été repositionné.

Les niveaux de sol sont concentrés dans le secteur au Sud de la ligne de blocs de marbre. L'état général de conservation des sols est très mauvais (Fig. 3.2.10). Qui plus est, il semble que le centre de l'espace au Sud se soit effondrés le long d'un axe Nord-Sud (Fig. 5.1.7).

La fondation de cet espace sud est composée de grandes dalles de gneiss rectangulaires équarries. Leur taille moyenne est de $86 \times 58 \times 19 \mathrm{~cm}$. Elles étaient en outre liés l'une à l'autre à l'aide d'un mortier blanc (identique à celui noté sur les blocs de marbre). Sur cette base, une couche de mortier rose a été posée. Il est assez grossier et les inclusions de dégraissant de TCA millimétrique sont bien visibles. Il sert de support à un hérisson de moellons en gneiss dont la taille varie entre $10 \times 5 \times 3$ et 5 x $3 \times 1 \mathrm{~cm}$. L'épaisseur de ces deux niveaux, noyés l'un dans l'autre, est d'environ $10 \mathrm{~cm}$. Ils sont recouverts d'un niveau de $5 \mathrm{~cm}$ d'épaisseur environ, composé de fragments centimétriques de TCA bien plats, noyés de manière irrégulière dans du mortier de même nature que le précédent. La régularité de sol ainsi créée a permis de poser un ensemble homogène formé de tesselles de marbre, de TCA et de fragments de céramiques relativement carrés ( $3 \times 3 \times 2 \mathrm{~cm}$ à $3 \times 4 \times 2 \mathrm{~cm})$. Sur ce tapis de tesselles, un niveau très fin (quelques millimètres) de mortier a été repéré à quelques endroits. Il est rose, à inclusions millimétriques de TCA, et très lisse (Fig. 3.2.11).

Le même schéma est ensuite répété : un hérisson de pierres en gneiss noyées dans du mortier, un niveau de fragments de TCA irrégulier, surmonté d'un autre niveau de TCA plus régulier et d'un niveau de tesselles. Au Sud, contre le mur et quasiment au centre de la pièce, la pose d'un panneau d'indication moderne a perturbé les niveaux de sol sur une cinquantaine de centimètre de diamètre et une trentaine de centimètre de profondeur. Sa stabilité ayant été fragilisée par la fouille, il a été retiré.

Les niveaux de sols empiètent de quelques centimètres sur les pierres de pourtour (Fig. 3.2.12). L'observation de la liaison entre les deux éléments a permis de constater que le niveau de mortier remonte légèrement, comme pour venir se coller sur un élément placé verticalement à l'avant du sol.

Dans la moitié nord de la Doric House, au-delà de l'alignement Est-Ouest des blocs de marbre, on trouve la continuité du sondage profond effectué par les suédois. Il n'a pas été vidé. Le nettoyage a révélé un radier de grosses pierres de calibre moyen $(8 \times 4 \times 2 \mathrm{~cm}$ à $16 \times 18 \times 4 \mathrm{~cm})$ et de fragments centimétriques de TCA organisés dans le sens EstOuest. A l'Est on retrouve, sur cet aménagement, un hérisson de pierres en gneiss noyé dans du mortier rose, sur lequel repose un niveau de fragments de TCA désorganisé et un niveau de fragments de TCA organisé, de même nature que ceux visibles dans la moité sud du bâtiment.

Enfin, la partie supérieure des murs a été nettoyée des petits blocs posés là, de la terre qui s'y était déposée et des herbes qui y avaient poussé.

\section{Interprétation}

Le travail de nettoyage et d'observation des vestiges a permis de comprendre trois points importants sur la Doric House, qui n'avaient pas été mentionnés dans les notes de l'équipe suédoise :

1) Les blocs rainurés de pourtour ont été interprétés comme des blocs de support de parapet. Cette hypothèse a été renforcée par la présence, dans les blocs en vrac, d'un marbre dont les rainures correspondent avec celles des blocs de pourtour. Il a alors été considéré comme appartenant au parapet, et re- 


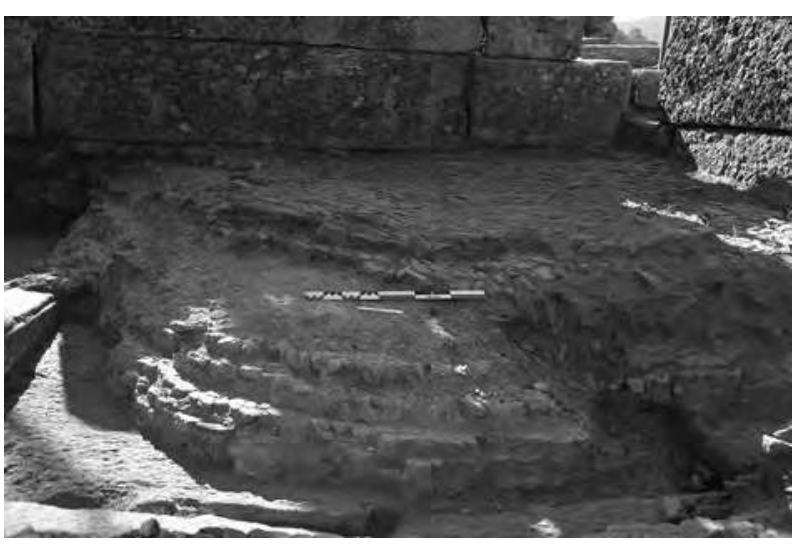

Fig. 3.2.10 : Vue générale de la stratigraphie et des niveaux de sol, moitié sud-est (M. Hauchart).

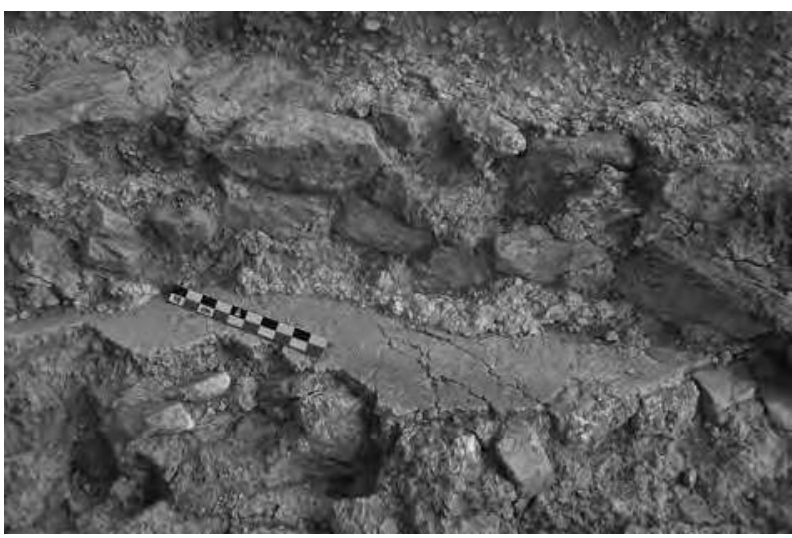

Fig. 3.2.11 : Détail du niveau de mortier hydraulique (état 1) (M. Hauchart).

placé à l'Est. La remontée effectuée par le niveau de mortier laisse également supposer la présence d'un élément vertical posé sur les blocs de pourtour, qui peut correspondre avec la présence d'un parapet composé de blocs de marbre. La remontée de mortier peut s'expliquer si l'on considère que les blocs étaient enduits du même mortier sur leur face intérieure. Les deux mortiers, de sol et du parapet, sont alors liés et forment un "bourrelet" de protection à l'endroit où ils se rejoignent.

2) En observant l'alignement central du même parapet, on constate que le bloc d'angle qui devrait se trouver à l'Est est inexistant. L'alignement semble s'être prolongé au-delà de l'actuel mur est du bâtiment. Lors de la fouille de la pièce des thermes contigüe, à l'Est, des fondations, distantes de quelques mètres du mur actuel de la Doric House avaient été repérées. Ces indices nous montrent que le bâtiment a été amputé de sa partie orientale et qu'un nouveau mur fut alors construit, à l'Est, sur l'aménagement d'origine. Sa fondation est de piêtre

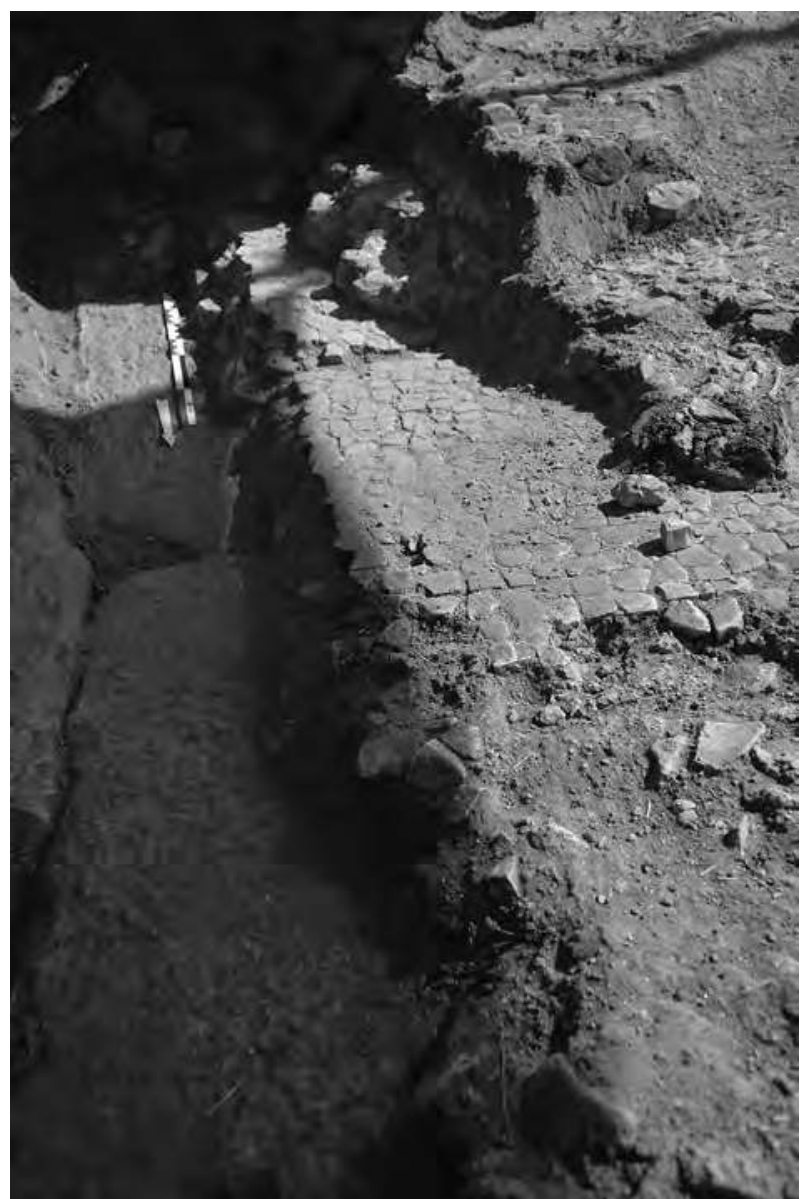

Fig. 3.2.12 : Empiétement des niveaux de sols sur les blocs de marbre du pourtour

(M. Hauchart).

qualité : blocs et pierres placés sans ordonnance, surmontés de grands blocs rectangulaires.

3) La superposition des niveaux de sol correspond à deux états distincts. Pour résumer, les niveaux se répartissent comme suit en partant du plus profond posé sur les gros blocs équarris formant base :

1. Hérisson de pierres en gneiss noyées dans le mortier ;

2. Fragments de TCA désorganisés noyés dans du mortier et/ou un niveau de fragments de TCA organisés ;

3. Tesselles ;

4. Niveau de mortier très fin.

Cet ensemble forme un seul aménagement. Les trois premiers niveaux sont des niveaux de préparation donc invisibles, alors que le mortier hydraulique constitue la couche supérieure, visible. Le schéma se répétant une deuxième fois et deux niveaux de mortier ayant été repérés, nous avons deux états de sol.

Le fait que la paroi interne du parapet ait été enduite de mortier, lié au mortier de sol et que ce 
mortier soit hydraulique permet de formuler l'hypothèse selon laquelle nous pourrions être en présence d'une retenue d'eau dans la moitié sud du bâtiment, alors que la moitié nord permettait un accès aux visiteurs et au bassin depuis le parapet.

Le nettoyage de l'espace et la fouille précédente de la pièce contigüe des thermes nous permettent d'affirmer, en chronologie, que la Doric House a été construite avant les bains est, ou tout du moins avant l'aménagement de la pièce nordouest des bains, dont l'aménagement a eu pour conséquence de réduire la superficie de la fontaine monumentale.

\section{CONSERVATION AND RESTORATION OF THE DORIC HOUSE}

(by Cr. Georgescu and G. Çimen)

The conservation and restoration work at the Doric House started on July $25^{\text {th }}$ and ended on August $17^{\text {th }}$. The team consisted of restorator Cristina Georgescu and archaeologist Görkem Çimen. During the second week, the team was helped by archaeology student Merve Günal.

As explained in the previous part of the report, after the 1950s excavations, the building was cleaned for the first time in 2017. The stone floor in the northern part as well as different layers of the floor in the southern part were uncovered. This archaeological work at the Doric House was followed directly by the conservation and restoration project. The primary aim of the project during the 2017 season, was to stabilize every layer of the floor in order to allow further progress.

\section{State of preservation}

The state of the preservation concerning the Doric House is briefly described, as follows: the building is divided into two where the northern area is separated from the southern area by a marble parapet in the east-west direction. The southern area which rests on gneiss blocks is also divided into two by a major central crack in the north-south direction of the building. The gneiss stone blocks are partly visible in the middle of the south-eastern and south-western parts. In this way, the Doric House, divided into three areas, resembles a T-shaped room. The mortar which was still visible on some parts of the gneiss blocks is identified as Hecatomnid, due to its similarity to the Hecatomnid mortar which was attached and visible on the marble parapet.

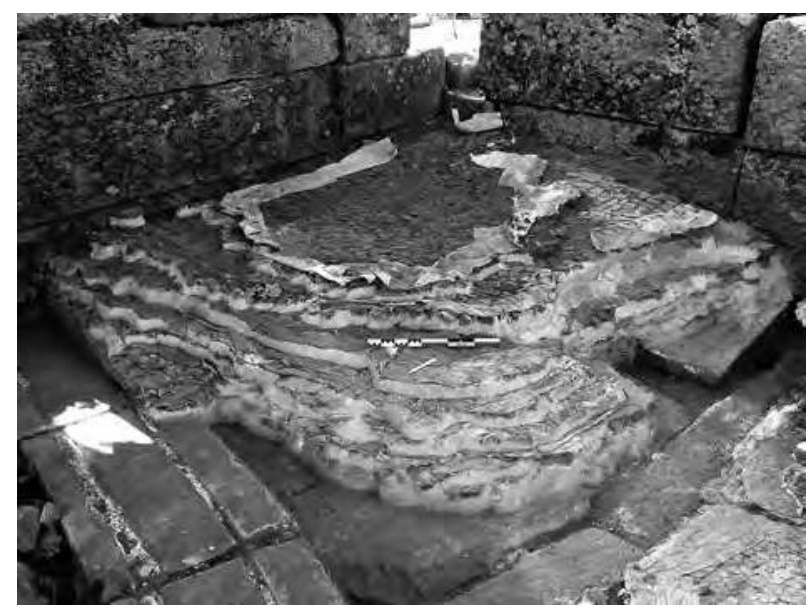

Fig. 3.2.13 : South-eastern part, showing the applied wet tissue papers and the applied mortar on the vertical edges (G. Çimen).

The color of the mortar is pure white which means that it might be pure lime ${ }^{35}$ as material. The height of the floor at the south-eastern part (Fig. 3.2.13), starting from the gneiss level, is around $0,5 \mathrm{~m}$. The area of the floor at the south-eastern part is around 14 square meters. As mentioned above in the previous parts of the report, the floor at the southern part of the building is stratified with different layers of tiles, stones, tesserae marble and mortar.

\section{Methodology}

Within this methodological introduction, three aspects should be mentioned:

Firstly, the choice of the methodology to preserve the floor of the Doric House depended on the building's outdoor exposure, the anthropic and accidental damages of the floor and the heterogeneous composition of the materials.

Secondly, weather conditions are of major importance for the conservation and restoration treatments concerning the length of the working period, usage of materials and choice of methodology. The conservation and restoration work at the Doric House was carried out in situ, which meant direct exposure to weather conditions. The specific conditions of the site during the summer of 2017 was a continuous wind, loaded with dust particles from the nearby quarries. The temperature during the working period of the project in 2017 was observed to have varied between $30^{\circ} \mathrm{C}$ and $39^{\circ} \mathrm{C}$ during the day with an average temperature of $36^{\circ} \mathrm{C}$.

Thirdly, we had to proceed to the cleaning of the same surface several times during the conser-

35) Air lime or Calcium lime made from pure lime (marble), with 5\% tolerated impurities. This type of mortar was also analysed and mentioned by Arnaud Coutelas on sample M12 from Andrôn A at Labraunda, in Henry et al. 2016: 350-354. 
vation and restoration work, using brushes and a domestic vacuum cleaner. This operation ensured de-dusting of the surfaces in order to prepare them for each of subsequent conservation and restoration treatment.

The chosen methods for our conservation and restoration work are shortly listed as follows:

1. Pre-work documentation by taking notes, measurements and photographs;

2. Treatment planning by choosing the treatments and techniques to use and putting them in order;

3. Treatments in form of stabilization, cleaning, consolidation, protection, exposure;

4. Post-work documentation with daily, weekly and final reports and photographs.

\section{Preparation and application of mortar}

A new lime mortar was prepared, from an original antique mortar saved from previous years in relation to the East Bath excavations at Labraunda, for the process of reinforcement which was the aim of the project. This preparation was carried out in several stages, as follows: firstly, the hydraulic lime powder was combined with water and left for a few days in order to obtain a better consistency; secondly, 1 liter of the slaked lime, together with some of its own water was taken to a basin and stirred with large trowels to remove possible particles in the lime. When the slaked lime reached its desired consistency without particles, 1.5 liters of crushed antique mortar was added in the basin. This new mixture was stirred until the crushed mortar pieces were well-mixed with the lime. These mentioned quantities of the materials in the mortar mixture was for one-time use in our work settings and this preparation of the mortar was daily repeated.

The application of this new mortar was carried out in four main steps:

- The surfaces of the horizontal and vertical edges of the floor layers were cleaned from soil and dust in order to reveal the material of the floor which were either tiles or stones. It was also done to find as much as possible of the original antique mortar in-between the material. Attaching the new mortar on the soil before cleaning it would otherwise result in cracks to be formed.

- These cleaned spaces between tiles or stones were afterwards wetted plenty with water and $5 \%$ ethyl alcohol ${ }^{36}$ before filling the spaces with the new mortar mixture. The result of the carbonation pro- cess of the mortar could have been affected without first wetting the surface.

- The applied new mortar was afterwards wellpressed, first with trowels and then, pressed again with Wishab ${ }^{37}$ sponges to obtain a flat, strong and rough surface, and also to avoid any possible cracks in the newly applied mortar.

- All intervened surfaces were covered after the application of the new mortar, in order to slow down water evaporation.

This was the main process for applying mortar throughout the restoration work.

\section{Conservation and restoration treatments}

The horizontal edges of every layer of the floor both in the south-western and south-eastern parts as well as the north-eastern area of the building were reinforced during the first week of the project. The process of this reinforcement started with cleaning the surfaces of the edges from dust and soil with brushes and continued with applying a solution on the edges. This solution consisted of Primal AC 33, diluted in $1 / 3$ of pure water and ethyl alcohol, which was used during the whole process, both to stabilize the surface and to stop the biological growth. The edges with the applied Primal solution were thereafter covered by two layers of wet tissue paper (as seen in Fig. 3.2.13). This first step was followed by reinforcing the vertical edges of all seven layers in the south-eastern part before applying the new mortar (a combination of slaked lime and antique mortar, as explained above) on them. The process of applying mortar started with covering the lowest layers first and continued upwards. After this, the following conservation and restoration work focused mainly on the south-eastern part of the Doric House.

The work continued in the second week of the project, by cleaning the whole south-eastern part and applying mortar on the vertical edges which were left from the previous week, namely on the south-western parts of the uppermost layer and the second layer from above. In this way, all the vertical edges of the south-eastern part had now been covered with mortar (Fig. 3.2.13). The conservation and restoration process thereafter moved on to the horizontal surfaces where the uppermost layer, identified as the tile floor, and the second layer had been our priority. This process consisted of five steps. The first treatment was to remove the wet tissue paper which during the first week was applied on the horizontal edges of the layers with

36) Alcohol is a surfactant that reduces superficial tension of the water.

37) A special sponge used in restoration operations for cleaning and aesthetic purposes. 


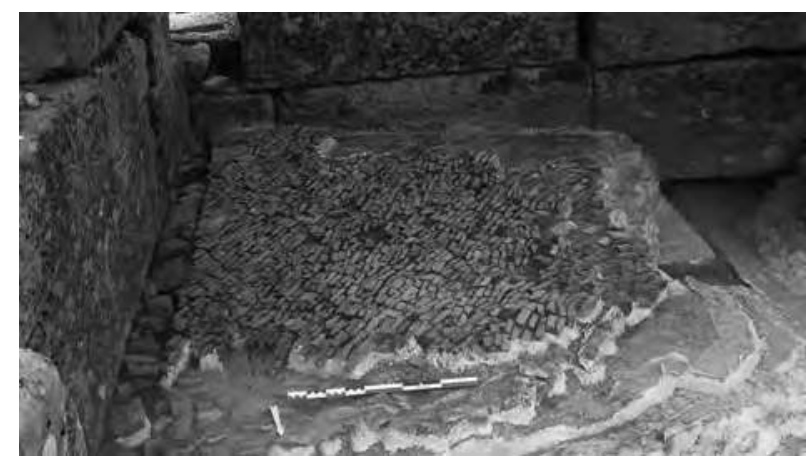

Fig. 3.2.14 : South-eastern part, after cleaning the surfaces of the tiles and spaces between them (C. Georgescu).

Primal solution. The second step was to clean the surfaces of the tiles and the space between every tile piece, using water, brushes and natural pine oil soap (Fig. 3.2.14). In order to clean between the tile pieces better, a vacuum cleaner was used. The next step was to apply Primal on the soil between the tile pieces to stabilize the surface. The fourth treatment was to apply Paraloid B72 (5\%) on the tile pieces. As the last step of this restoration process on the surfaces, the new mortar started to be applied in the spaces between the tiles of the two uppermost layers, using a tiny trowel. The new mortar was applied until just below the top parts of the tile pieces on the floor layer. Beginning from south and going northwards at the south-eastern part, the spaces between every tile piece of the uppermost layer of the tile floor and the second layer from above were at the end of the week completely filled with mortar. Apart from this, the space between the tile floor on the uppermost layer and the south wall of the building was cleaned, by removing soil until finding the original antique mortar and then wetting the area and applying Primal on the surface. The space was thereafter filled with mortar (Fig. 3.2.15).

During the beginning of the third week of the project, the work was stopped at the south-eastern part for one day to allow us to better understand the south-western part. The south-eastern side of the south-western part needed to be removed to see if the "marble floor" as a preparation level continued under the three remaining layers of the flooring (Fig. 3.2.16). These layers were unearthed as follows: the uppermost layer consisted of tiles which resembled the tile floor of the south-eastern part. The second layer consisted of stones, mostly on its eastern area and there was a compressed white mortar on the stones in the middle of this layer. On the west side of this mortar there were tile pieces that emerged instead of the stones, which were the main

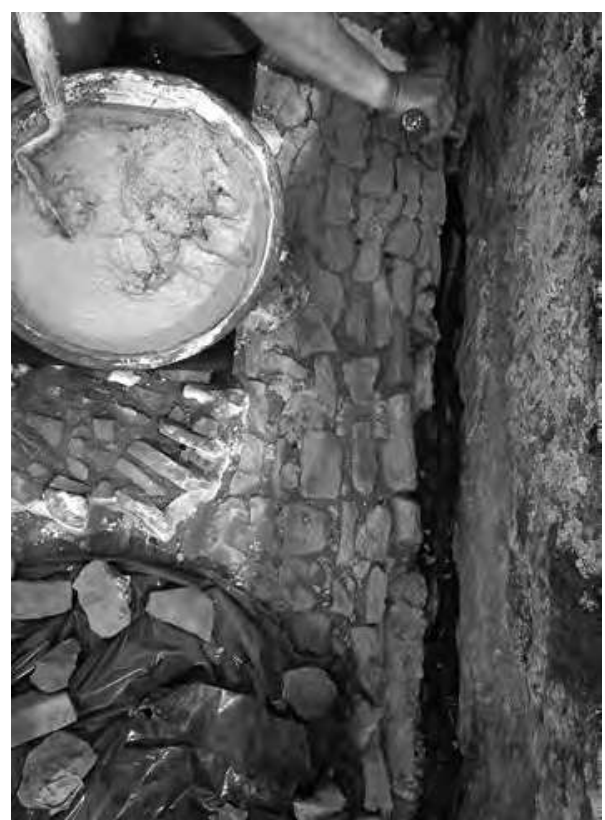

Fig. 3.2.15 : South-eastern part, when applying mortar in the space between the south wall and the tile floor (G. Çimen).

material of this layer. In other words, the stones were replaced by tile pieces in some spots which were similar to the uppermost layer. This way of using stones and tile pieces on the same level of the floor indicated a mixed style of two materials. Amongst the tile material of the second layer, 8-10 fragments of roof tiles were found. The lowermost layer also consisted of stones, as in the second layer. After the removal of these three layers, it was observed that the marble preparation level continued underneath the lowermost layer of stones in order to have a flat surface below the other layers (Fig. 3.2.17). A large and deep crack in the middle of this marble preparation level was also revealed when the layers were removed.

During the fourth and last week of the project, the process of applying mortar between the tiles and stones of all the horizontal layers on the south-eastern part, was totally completed. The original antique roman mortar, pinkish in color was well-visible especially on an area right below the second layer of tiles from above, and between the stones and tesser$a e$ on the third and fourth layers from above. For the purpose of reinforcement, resin was applied twice on these surfaces of antique roman mortar which were decided to be left in situ.

As the last step of the conservation and restoration, all the tile pieces of the tile floor and second layer from above as well as the visible stones of all layers on the south-eastern part were cleaned 


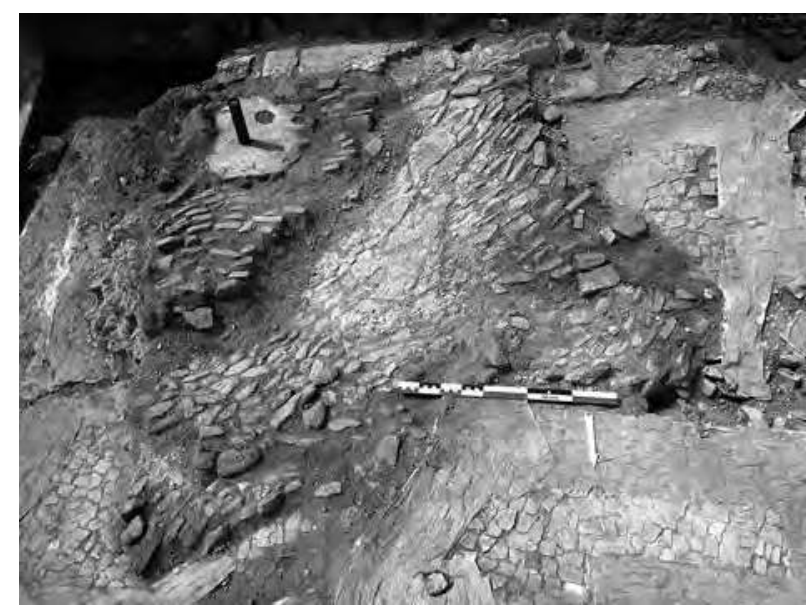

Fig. 3.2.16 : South-western part, showing the different layers (G. Çimen).

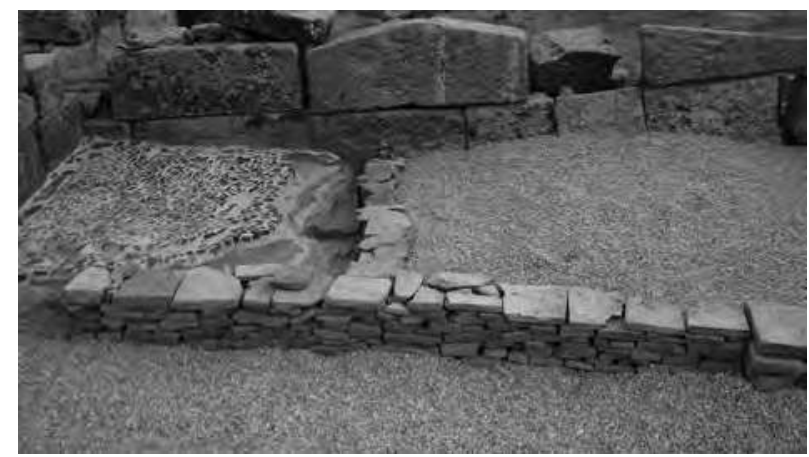

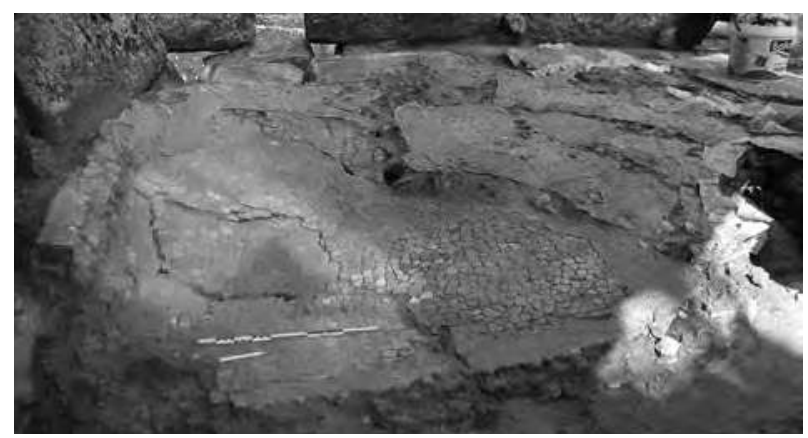

Fig. 3.2.17 : South-western part, marble preparation level after the removal of the three layers (C. Georgescu).

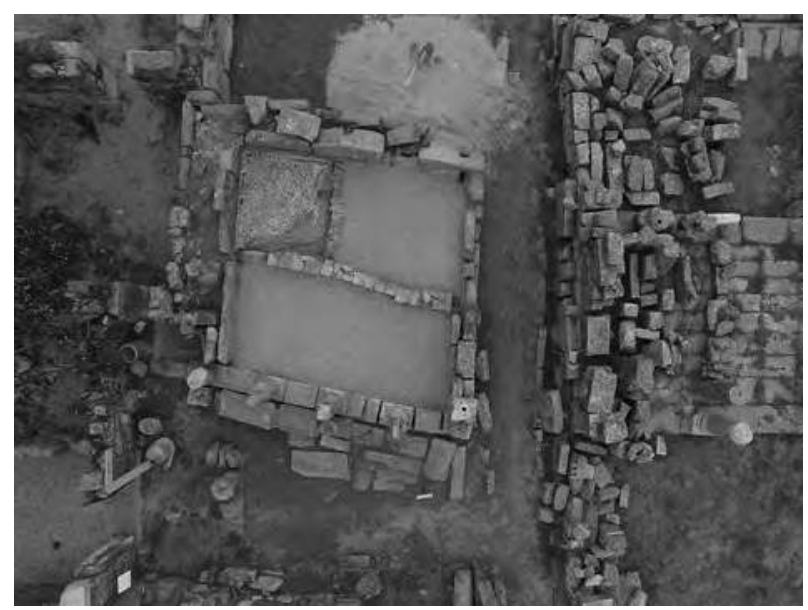

Fig. 3.2.18-19 : The Doric House after the conservation-restoration work (C. Georgescu).

with natural pine oil soap and solution of acetic acid ( $2 \%)$. After the tiles were dried, Paraloid B 72 was applied on them for the final protection.

\section{Conclusion}

During the period of one month, multiple processes of conservation and restoration work were carried out in order to primarily stabilize the floor of the whole south-eastern part, which is equal to a quarter of the Doric House. This part of the building remained in open-air. To protect the other parts of the building, other arrangements were done. Tile fragments from the M-terrace were carried down to the Doric House to construct a wall on the gneiss stone blocks between the two parts in the south of the building. This was done to separate the restored south-eastern part from the partly restored south-western part. Another wall, using ancient brick fragments, separating the northern and the southern parts of the building was also constructed on the marble parapet in the east-west direction. After the construction of these two walls, the south-western part and the northern area of the Doric House were cleaned and covered, first with geotextile and then with pebbles. With this, the conservation and restoration work at the Doric House was completed (Fig. 3.2.18-3.2.19) and the goal of the project for the 2017 season was reached.

\subsection{ANDRÔN A (par O. Henry)}

La pièce centrale de l'Andrôn A d'Idrieus, bâtiment emblématique du site, a été fouillée au cours des saisons 2014-2015 $5^{38}$. Ces fouilles ont permis de mettre au jour des niveaux de sol datant de la période de construction du bâtiment, autour du milieu du $4^{\mathrm{e}} \mathrm{s}$. av. J.-C. Immédiatement après les fouilles, le sol ainsi mis au jour avait été protégé par une couche de géotextile recouverte de sable. L'expérience a prouvé que cette mise en place était loin d'être satisfaisante puisqu'elle avait tendance à conserver l'humidité au sein de la pièce, ce qui provoquait le développement de plantes vertes persis-

38) Voir les rapport donnés dans les volumes correspondants (2015 et 2016) de Anatolia Antiqua. 


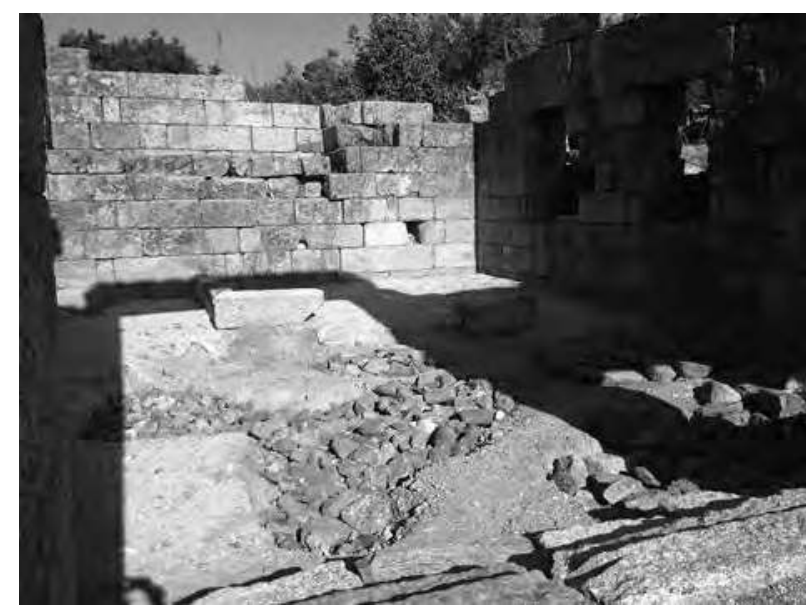

Fig. 3.3.1 : Les fosses tardives de l'Andrôn A remblayées $(\mathrm{O}$. Henry).

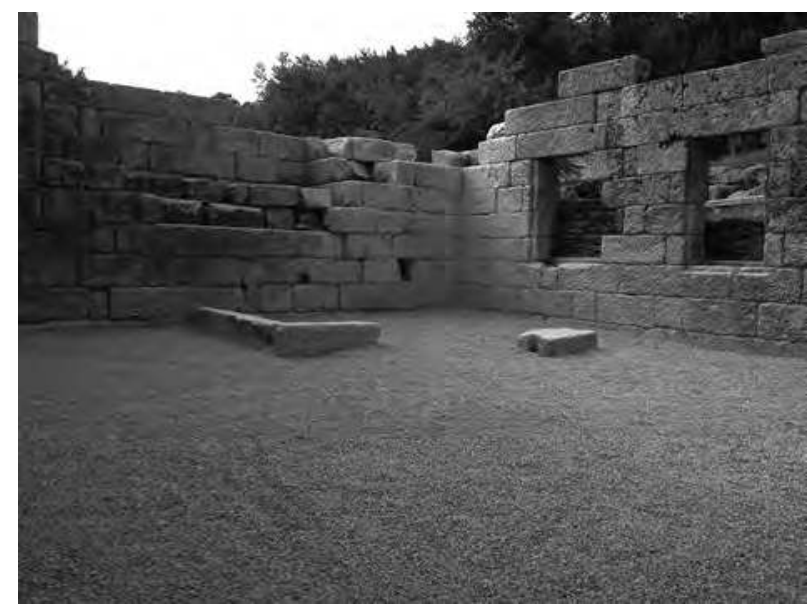

Fig. 3.3.3 : Le naos de l'Andrôn après la couverture de gravier (O. Henry).

tantes particulièrement indésirables pour la conservation des sols antiques.

Cette année il a donc été décidé de changer la protection de ces derniers. Ainsi, nous avons commencé par remblayer, à l'aide de pierres, les nombreuses fosses "tardives" creusées au cours de la période byzantine pour servir de réceptacle à autant de pithoi dans le cadre de la transformation de l'Andrôn en huilerie (Fig. 3.3.1). Ensuite, nous nous sommes procurés une nouvelle formule de géotextile (Typar

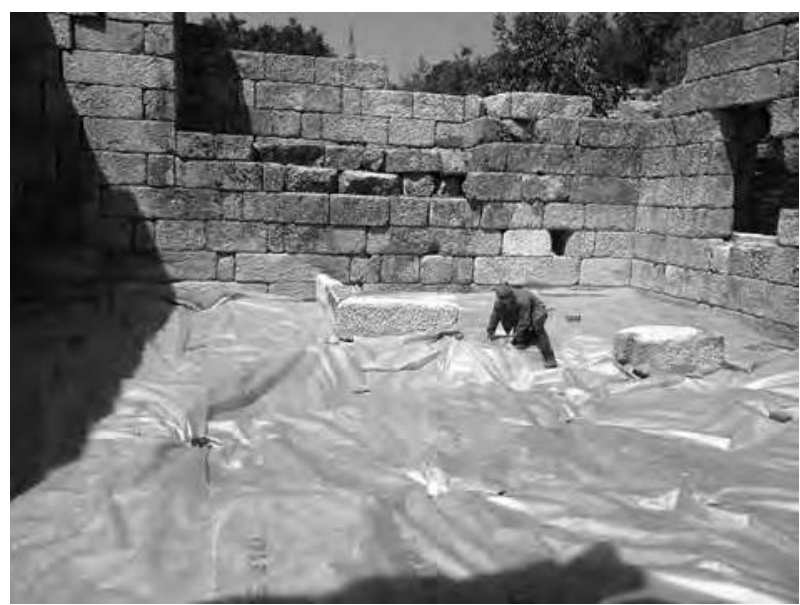

Fig. 3.3.2 : Pose du revêtement géotextile dans l'Andrôn A (O. Henry).

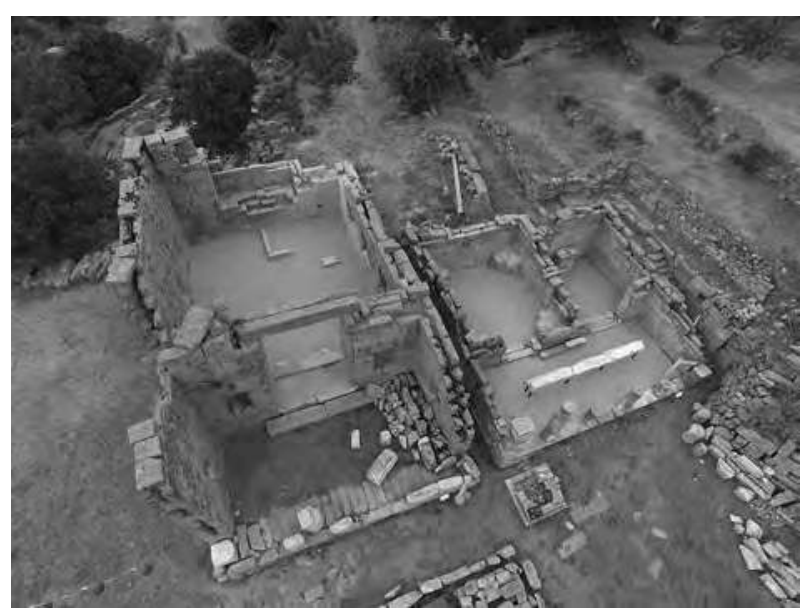

Fig. 3.3.4 : L'Andrôn A et les Oikoi à la fin de la saison 2017 (O. Henry).

SF27), bien plus efficace contre la poussée des plantes et dont la resistance aux intempéries a été démontrée. Après la pose de ce niveau de géotextile (Fig. 3.3.2), l'ensemble de la pièce a été recouvert d'une épaisse couche (20 à $25 \mathrm{~cm}$ d'épaisseur) d'un gravier blanc identique à celui qui avait été déposé dans l'église est et les Oikoi en 2016 (ainsi que dans la maison dorique en 2017). Ce gravier présente le double avantage de ne pas pénétrer le géotextile et de ne pas retenir l'humidité (Fig. 3.3.3 - 3.3.4).

\section{SOL PROJECT (by Axel Frejman)}

The Surroundings of Labraunda (SOL) project is a small scale survey project that aims to document remains of human activity outside the temenos of Zeus Labraundos. The survey was conducted dur- ing the period 2014-2017 and involved a total of 19 weeks in the field with 1-4 persons working on the project (Fig. 4.1.1). ${ }^{39}$ Survey result are integrated into the author's research towards a doc-

39) I would like to thank Görkem Çimen, Ludvig Jungeström, and Jenny Radon for their arduous work with the project, and for not 
toral degree investigating activities in extra-temenal areas of large rural sanctuaries in Greece and western Asia Minor, 700-200 BCE. The 2017 field season was the final season for the project in its current form.

The aim of the 2017 season was to document a selection of structures and rock cuttings that had been found in the survey, as well as investigating the distribution of spring houses and possible road network around Labraunda, and furthermore to correct a few minor errors that had been identified in the data from previous field seasons. Much of the work was focussed on clearing and documenting a large structure roughly 500 metres south of the sanctuary, labelled the JG Building, see below. Also, a systematic reinvestigation and integration of previously published and new spring houses was done. One of which (SH18) was documented closer. Furthermore, the possible route network around Labraunda was investigated.

\subsection{JG BUILDING}

The JG Building is located on an outcrop overlooking the dell and valley below, it also has direct visual contact with the acropolis fortress, the split rock at the sanctuary, and several of the forts around Labraunda ${ }^{40}$. The outcrop has steep slopes to all sides but north, where it opens up to a field. The remains that are visible today are heavily destroyed, but a number of walls can be followed in the rubble, see Fig. 4.1.2. The dry masonry walls are built of mostly unworked blocks, however some blocks with a specific function -threshold and doorposts for example- have been worked. Where wall thickness can be measured it falls into the range of $0.60-0.70 \mathrm{~m}$. The layout of the structure is roughly square, with a courtyard to the south and an annexed structure to the southwest. The main body of the structure measures E-W 12.5 by N-S $10-11.5 \mathrm{~m}$. With the courtyard added the total length of the structure is $21.5 \mathrm{~m}$. Where the building technique can be discerned the walls are made up of two faces of stretchers, bound to- gether by headers. On the lowest course of the outer walls large orthostats are used on the outer face of the wall while smaller rubble is used on the inside $^{41}$.

Six defined spaces can be observed today, at least three of which (1, 3, and 4) we might expect to have been roofed. The wall thickness would suggest that there was a second storey, furthermore two blocks with beam hole cuttings were found that could have supported the second floor ${ }^{42}$. However, the second storey might not have been present in the whole structure. The layer of collapsed blocks is considerably thicker around spaces 1 and 3. Furthermore, an apex block was found in space 1, we can expect the walls of either space 1 or 3 to have been built of blocks up to roof level. If there was indeed a second storey it would only have covered spaces 1 and 3, probably only space 3. In space 2 the collapse is much less visible, this might be explained by it being an unroofed inner courtyard with walls partially made up of perishable materials, or it might have been cleared at a later stage, see below. There is a drain in the east wall of space 2 which would indicate that this space was at least partly unroofed. The expected layout would be to have a covered portico facing south, with rooms behind or on the sides ${ }^{43}$. There are two blocks in the northern part of space 2 -running in a north-south direction- that could constitute a narrower inner wall, but they could also be remains of a basin or a platform. There are however no indications of walls running in an east-west direction. Nevertheless, we can expect there to have been roofed spaces along one or more of the outer walls of space 2, resting either on columns or on walls ${ }^{44}$. A tentative reconstruction of the spatial layout can be seen in Fig. 4.1.2. The situation is further complicated by the fact that the wall between space 2 and 4 might be later than the rest of the structure ${ }^{45}$. There might therefore be two phases of construction using the same building technique here, presumably chronologically closer than the rebuilding of the east

deserting at the first sight of Labraunda's unforgiving terrain. I would also like to thank the funding bodies that have made this project possible: Helge Ax:son Johnsons Stiftelse, The Royal Swedish Academy of Letters Enboms donationsfond, Anna Maria Lundins Stipendiefond, Societas Archaeologica Upsaliensis Forskningsråd, Uppsala university travel and research grants: Sernanders, Axel W Perssons.

40) For an overview of the fortifications around Labraunda see Karlsson 2011.

41) The building technique is very similar to that of the farmstead at Vari, see Jones et al. 1973: 358-359 and pls. 65-67.

42) Jones et al. 1973: 425-426.

43) This seems to be the preferred layout, examples of southwards facing pastas or porticoes can be seen for example in Olynthos, Priene, and Athens, see Jones et al. 1973: 419-420, 430-434 and fig. 16.

44) As there is no shortage of space around the building there would be very limited gain in only having a walled in courtyard, we must expect the space to have provided shelter from the elements.

45) The blocks of the north wall in space 3 all end in the outer corner and show no indication of being joined with the north wall of space 4. It should therefore have been abutting against the northeast corner of space 3 . 


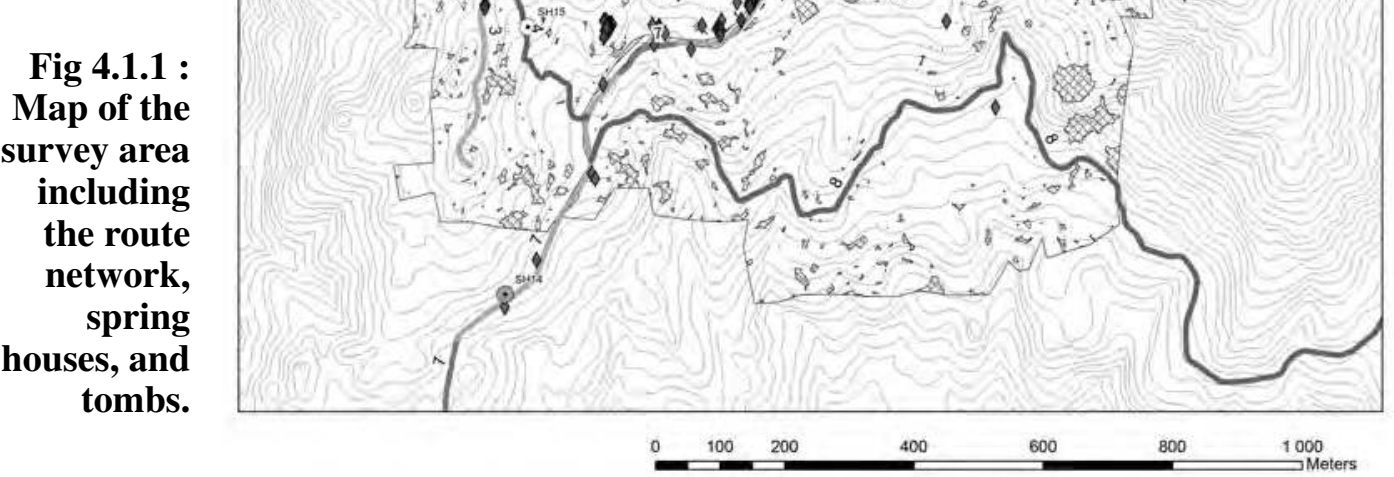

Fig 4.1.2 : Actual state plan of JG

Building, grey indicating blocks that are in situ or can be identified as having shifted out from the walls, dashed line suggested walls and roofed space with only minor evidence present.

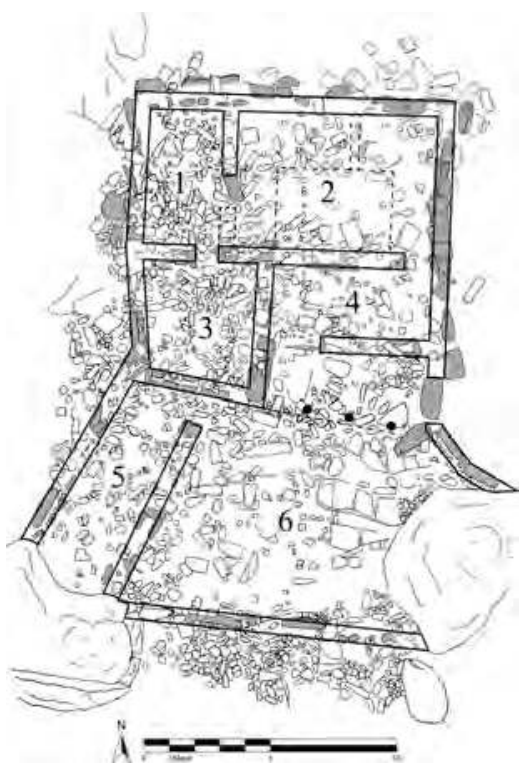

Fig 4.1.3 : Spring House 18. West dromos wall (left), north wall with spring chamber (middle), east dromos wall with shaft (right).

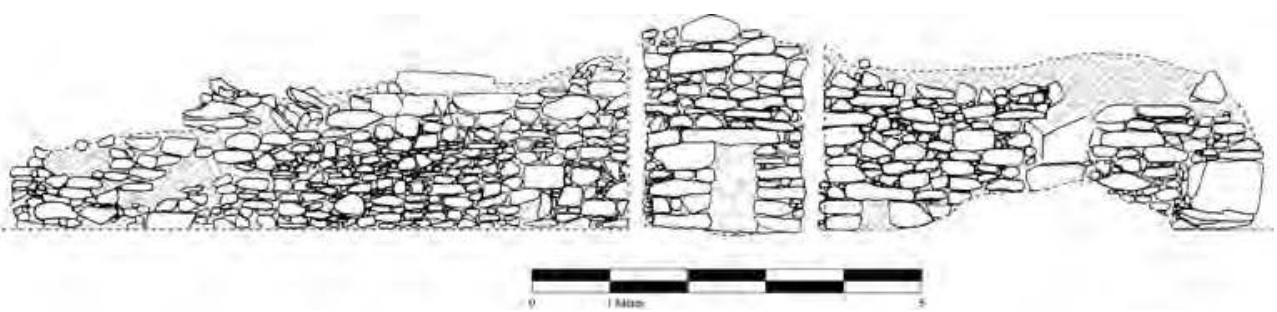


façade in a different technique, see below. Space 5 is a roughly $2.8 \mathrm{~m}$ wide elongated space. There is no door between space 3 and 5, the most likely opening into space 5 is found in the north end of its east wall. The thickness of the walls could support a second storey and it is possible that there was a door between space 3 and 5 on the second storey. The ground floor of space 5 would then be connected with space 6 and the activities therein, whereas the second floor would be connected to space 3 . Space 6 is a courtyard held up by a southern terrace wall. The level of the ground in space 6 is today roughly three metres below that of space 1. However much of the terrace wall at the southern side of space 6 has collapsed and we should expect the level to have been considerably higher in antiquity. The bedrock in space 6 does not show marks of quarrying, but is broken off in a way that shows that the space was used to quarry blocks for the construction of the structure.

The east façade is the most well preserved, it also differs from the other walls. Its upper courses are built with flat blocks lying horizontally on top of each other rather than placed vertically. The blocks are of the same type as in the rest of the structure, but laid out in another technique. This indicates that the wall was rebuilt at a time when at least parts of the original structure had collapsed.

Considering the placement the structure should probably be identified as a farmstead. The field adjoining to it is the largest uninterrupted field in the immediate vicinity of the sanctuary, some 5.5 hectares, closely to the south there is an additional $c a$. 5 hectares of fields. It is thought that a family farm would have had around 3.6 to 5.4 hectares of land connected to it, enough to support a nuclear family ${ }^{46}$. Though in some areas the farms were considerably larger and presumably both required more labour and supported more people. In Halieis cautious calculations give a range between 5.5 and 22.5 hectares, and at Chersonesos in the Crimea most farms were 27 hectares ${ }^{47}$. This puts the present structure's potential fields -roughly 10 hectares- at two to three times the size of what would be expected for a single family, but not in any way an extreme areal.
That the location would be strategically highly suitable for military purposes is conspicuous. The wall thickness is similar to that used for the adjacent rooms at Burgaz and Tepesar Kale (ca. $0.70 \mathrm{~m}$ ), but the fortified parts of these forts have a wall thickness of 1.30-2.0 $\mathrm{m}^{48}$. In a defensive structure of this size we would expect at least parts to have been built with thicker masonry. Conversely, if the main concerns were the practicalities of farmstead activities one would expect the structure to be situated in a less exposed location -both concerning visibility and weatherand closer to a source of water. The JG Building thus eludes easy classification. The location suggests a connection to the defence network around Labraunda, but the structure itself suggests a farmstead. However, it has been suggested that there was a connection between defence and agriculture in Eretria, the importance of the products necessitating integration between the $\mathrm{two}^{49}$. We might explain the JG Building as a similar case of integration between farming and the defence network at Labraunda.

No clearly datable material was found during the cleaning of the structure. The use of header and stretcher technique in the walls indicates a terminus post quem in the classical period. The defensive network around Labraunda was extended in the late $3^{\text {rd }}$ century $\mathrm{BC}$, the dated material from the survey also points to an increase in activity in Labraunda's surroundings in the $3^{\text {rd }}$ century $^{50}$. The JG Building is unlikely to be earlier than $3^{\text {rd }}$ century. Possibly, it was part of the fortifications' building programme, but in our current state of knowledge, it is too early to say. For features identified as farmsteads there is a predominance in two timespans, late $6^{\text {th }}$ to $3^{\text {rd }}$ century BC, and Late Antiquity ${ }^{51}$. Roman farmsteads tended to be connected to much larger fields than their Greek counterparts, the building technique -no concrete and rather large building blocks- is also more reminiscent of Greek or earlier Roman structures than Late Roman ones ${ }^{52}$. The rebuilt east façade could be evidence of a second phase of use in the Late Antique period.

46) Jameson 1992: 137.

47) Jameson 1992: 139, 141.

48) Karlsson 2011: 217-224. Ucalan kule has wall thicknesses similar to the JG Building, though it can be explained as a cost cutting measure in this small lookout tower, B. Vergnaud oral testimony.

49) Eretria XXI: 275-276.

50) Henry et al. 2017: 219 .

51) Winther-Jacobsen 2010: 270 drawing on Alcock 1993; Morris and Papadopoulos 2005: 164 with references in note 43.

52) Alcock 1993: 59. 


\subsection{ROUTE NETWORK}

The main roads from Labraunda towards Mylasa and Alinda have been charted previously, the possible routes around the site have received less attention ${ }^{53}$. Possible routes around the sanctuary were investigated to better understand the movement patterns in the surrounding areas. As very few actual traces remain of either a pavement or supporting terraces for roads the method had to rely on other features -tombs and spring houses for example- but also on the topography. The terrain might have changed considerably since antiquity, however the obstacles in the terrain around Labraunda usually come in the form of large boulders or steep rocky slopes, both of which can be expected to have been relatively stable through time. The criteria used when charting the routes was that it should have been possible to travel with a cart. The presence of tombs and spring houses are considered indicative of a route passing nearby. Furthermore some significance is given to the placement of present paths, not only because the terrain around Labraunda does not easily admit routes to be drawn, but also because once created travelling routes tend to be very static elements in the landscape, not changing unless an external force compels them to. These criteria leave out the smaller routes that probably crisscrossed the landscape. The possible ways such smaller routes might have taken are infinite, and it would not be sensible to try to identify them. Smaller routes have been documented were the evidence strongly suggests the presence of such, at present confined to route 1 and 6 .

Route 1 is found north of the acropolis. The evidence for it is the combination of five spring houses, two of which are most probably of ancient date, a long terrace of uncertain date holding up the path, a staircase cut into the bedrock, and the topography which makes this a preferable route from the acropolis towards the north. It can be followed with some certainty for about $110 \mathrm{~m}$, but would -in the south end- have continued up to the acropolis along a still unknown route. In the north end, the path passes down a rock cut staircase to a more level space, after this there is no indication for the direction of the path.
Route 2 was investigated in 2016 but received further study during 2017, it is a larger route that would no doubt have accommodated cart travel ${ }^{54}$. It runs west of the sanctuary, through a structure in the northwest identified as a gate. At the other end it passes by the western end of the stadion, after which it can no longer be followed with any certainty. Today it connects to a path leading up from the main road, through the ancient stadion, to the modern village just west of the sanctuary. This path was built in the 1960s and does not appear to have had an ancient precursor ${ }^{55}$.

Route 3 connects to route 2 and leads along a ridge to Ucalan kule. It was bulldozed when building the pylons for the power lines leading over the mountains, one of which stands next to Ucalan kule. There is good reason to believe that there was an ancient path in roughly the same line. The steep topography and rocky terrain makes it almost impossible to get to Ucalan if not along the ridge. As it forks of from Route 2 there are also three tombs aligned in the direction of Ucalan.

Route 4 is a tentative connection between the main road (7) and route 2 . The evidence for a route in this line is circumstantial; route 2 must at some point have connected to the main road. The indicated line could accommodate a cart, and runs straight past a spring house. The slope is gentle, and considering the terrain around Labraunda, the fact that cart travel would have been possible makes for a quite strong argument in itself.

Route 5 is the main road from Labraunda over the mountains towards Alinda. In four parts the terrace upholding the road is visible ${ }^{56}$. It is furthermore visible as a depression in the landscape in several parts, passes by three spring houses, several tombs, and also makes a turn around the East Building, located close to Spring House 36. There should be little doubt about the validity for the stretch between the East Building and the visible remains furthest to the east. Between the sanctuary and the East Building the route is a bit harder to follow. Most probably the route followed the natural glade gently sloping towards the sanctuary, although it is not possible to say exactly where the line of the road was because of later terracing and agricultural transformations. The other possibility would be that the route continued along the line of route 6 , but this is less probable.

53) Main roads see Baran 2011; The routes around Labraunda have been under investigation by P. Lebouteiller as part of the topographic work at the site. The routes have not been treated separately but parts of them have been included in Henry et al. 2015: 309-310 and fig. 6. Lebouteiller's and my results are in agreeance.

54) Henry et al. 2017: 202-204.

55) Oral testimony by the site guard Ali who grew up in the area, the reason for building the road apparently being that was two persons bought jeeps and wanted to drive up to the modern village.

56) Two of these are included in Baran 2011: 64-65. 
Route 6 is short stretch forking off from route 5 , it can be conjured for some $130 \mathrm{~m}$. This route is smaller and could not have sustained cart traffic. It runs next to an area of rock cuttings that could be identified as a commercial area. There is no clear continuation towards the west. It is surrounded on almost all sides by impassable terrain, but could have reconnected to Route 5 as a footpath further to the west. There is wider track leading up from the modern road in this direction that could connect to route 6 , it is however unlikely to have an ancient precursor because it cuts through ancient remains.

Route 7 is the main road from Labraunda to Mylasa. Ancient remains are visible in several spots and more is hidden beneath the modern asphalt road. It has been discussed in writing before and no new information has come forth to make it necessary to discuss it again here ${ }^{57}$.

Route 8 is in use today as a dirt track, any evidence of an ancient precursor would have been destroyed at the time when the route was bulldozed, presumably to make it wide and flat enough for motorized traffic. There are scattered remains along the line of the road but no spring houses and only a few tombs. There could possibly be an alternate route leading down from the sanctuary to route 8 , following the tombs in the landscape apparently laid out in a line towards the south. However, the terrain in this area is today very unforgiving and it is difficult to form a clear picture. If there was a route along the line of tombs, it could not have been more than a footpath. Where the hypothetic route 8 would lead is unclear, based on the number of tombs and other remains close by it does not appear to have been in as lively use as the main roads to Mylasa and Alinda. However, if there was a route from Labraunda towards the southeast this would be the most likely candidate.

\subsection{SPRING HOUSES}

An inventory of the spring houses around Labraunda was done in 2017, integrating the previously published examples with the data from the survey, see Fig. 4.1.1 ${ }^{58}$. There were some uncertainties in the publication necessitating an inves- tigation of the in situ remains. The spring houses were located and documented by GPS and photo $^{59}$. In cases where there was uncertainty about the nature of the remains, they were not included in the new list of spring houses ${ }^{60}$. Furthermore, it could be established that Spring House 41 has been eradicated in the widening of the road close to Labraunda, it is still included in the list ${ }^{61}$. Continuing with the same numbering system, the previously unknown spring houses that have been documented in the survey were added to the list, numbers SH43-SH53. The newly documented examples conform to the same shape and function as the previously published examples, except for Spring House 53 that is the monumental fountain discovered in $2013^{62}$.

As a step towards a more detailed understanding of the spring houses around Labraunda, Spring House 18 was drawn during 2017, see Fig. 4.1.3. It is representative of the many rubble wall spring houses that have been documented in previous efforts during the excavations, and within the SOL project ${ }^{63}$. It was not deemed worthwhile to make a stone plan for the structure as the upper side of the blocks in the wall are covered with earth ${ }^{64}$. The back wall has a covered chamber which housed the water, it is dry today. From the back wall the dromos walls run at a right angle, forming an elongated $\Pi$-shaped structure, the lengths of the walls are west wall $7.9 \mathrm{~m}$ and east wall $5.6 \mathrm{~m}$ respectively. The length of the dromos walls is among the longest examples documented, the mean length at Labraunda being around $4.6 \mathrm{~m}^{65}$. In the eastern dromos wall, close to the back wall, there is a shaft going straight into the wall at ground level. Its length can be measured for roughly $1.8 \mathrm{~m}$, until the shaft makes a turn and can no longer be measured. None of the spring houses around Labraunda have been excavated and we can only speculate on the date. However I share the view presented by Anneliese Peschlow-Bindokat that the size of the blocks can be used as an indication for the date, larger blocks indicating an earlier date ${ }^{66}$. Spring house 18 has rather large blocks around the spring chamber and could well be from pre-byzantine times.

57) For the Sacred way, here route 7, see Westholm 1963: 8-9; Hellström and Karlsson 2005: 77-78, 80 fig. 4; Hellström 2009: 270; Baran 2011: 52-64.

58) Baran 2011.

59) SH1-14 were not checked.

60) The examples that could not be vindicated are Baran's n ${ }^{\circ} 28,29,35,37,38,40$, and 42.

61) It was a well preserved and still functioning example, situated next to the road prior its widening, Baran 2011: 89-90 and fig 84.

62) Henry et al 2014: 269, 272.

63) Many spring houses are published with location and rough shape in Baran 2011, but no drawings of the walls are included.

64) For a schematic plan see Baran 2011: 75 pl. $3 n^{\circ} 18$.

65) Baran 2011: 95-96 table 1.

66) Peschlow-Bindokat 1996: 45 fig. 57, 47-48. 


\section{DOCUMENTATION}

\subsection{PHOTOGRAMMETRIE}

L'objectif de cette mission était de réaliser la photogrammétrie de monuments du site et de fouilles en cours afin de continuer la documentation initiée en 2015. L'appareil photographique utilisé est un reflex Nikon D80.

\section{Les Monuments du site}

\section{- La Stoa Nord}

Modèle 3D effectué avant la fouille de la partie est de la stoa. Le but étant d'avoir dans le modèle 3D tous les blocs, colonnes et éléments d'architecture effondrés sur place. L'étude de leur emplacement pourrait alors être menée postérieurement ou parallèlement à la fouille. La première session a été menée afin d'avoir une vue d'ensemble de l'état du terrain avant le premier nettoyage et la fouille en elle-même par des prises de vue au sol mais également par drone (Fig. 5.1.1).

\section{- La Stoa Est}

L'extrêmité nord de cette stoa a été nettoyée dans les premières semaines de cette campagne, soit deux pièces pour une surface d'environ $80 \mathrm{~m}^{2}$. Comme de très nombreux blocs se sont écroulés sur place lors de sa destruction, beaucoup sont encore sur place. La photogrammétrie de cette zone a été effectuée en plusieurs sessions en raison de la surface importante à couvrir mais aussi parce que les blocs effondrés sont fastidieux à photographier sous le maximum d'angles. Seule la partie nettoyée est concernée par la photogrammétrie au sol (Fig. 5.1.2). En revanche, une seconde session a été menée par drone, ce qui a permis d'avoir une vue de l'ensemble de la stoa.

\section{- L'inscription de l'Oikos}

Trois sessions de photos ont été choisies pour tenter d'avoir le moins d'ombre sur les six faces de cette grande inscription sur marbre, installée désormais sur un support métallique pour en faciliter la lecture. Le plus difficile est de faire le lien entre les différentes sessions photographiques puisque les photos doivent pouvoir se superposer et la différence de lumière peut nuire à la reconnaissance par le logiciel (Fig. 5.1.3).

\section{- La tombe monumentale}

La tombe étant fermée à l'exception de l'entrée, la lumière manque. Le soleil à son zénith est le meilleur moment afin qu'il n'envahisse pas les pièces et provoque ainsi de forts contrastes. Plusieurs sessions ont été nécessaires pour l'intérieur de la tombe, la cour d'entrée, l'étage supérieur, les trois autres façades et le toit. Au total, 2140 photographies ont été traitées à Paris sur un ordinateur plus puissant (Fig. 5.1.4).

\section{Les fouilles et nettoyages en cours}

En parallèle aux prises de vue sur le terrain, des modèles 3D ont été générés, soit en nuages de points soit en maillage. Deux procédés ont été utilisés : le premier, en moyenne résolution, qui se limite à créer un nuage de points dense afin de vérifier si les prises de vues ne sont pas à compléter. Le second, qui nécessite de créer d'abord un maillage à partir du nuage dense, puis de générer une orthomosaïque.

\section{- Sondage S17}

Dix levées ont été réalisées au cours de la fouille du sondage à l'Ouest des bains est par les responsables de la fouille et par moi-même pour la dernière levée dont l'ortho-photographie a été générée (Fig. 5.1.5).

\section{- Le sondage BTC Zone 1}

Le sondage BTC Zone 1 au pied de la tombe monumentale a été également le sujet d'une photogrammétrie (547 photos, 46.700 .000 environ de points pour le nuage dense) à la fin de la fouille (Fig. 5.1.6).

\section{- La maison dorique}

Après le dégagement et le nettoyage des sols du bâtiment, la photogrammétrie a été réalisée juste avant la restauration des sols. Les clichés sont précis car les différentes strates de préparations de sols et mortiers doivent être visibles (Fig. 5.1.7).

La campagne 2017 qui s'est déroulée sur deux semaines m'a permis de documenter plusieurs monuments qui n'étaient plus en cours de fouille à mon arrivée comme les stoa nord et est, la tombe monumentale mais également l'inscription de l'Oikos. A la suite des nouvelles découvertes, j'ai documenté le sondage S17 à l'Ouest des bains de l'est, le sondage BTC zone 1 et la maison dorique en cours de restauration. La prochaine campagne me permettra de continuer le programme de documentation 3D. 


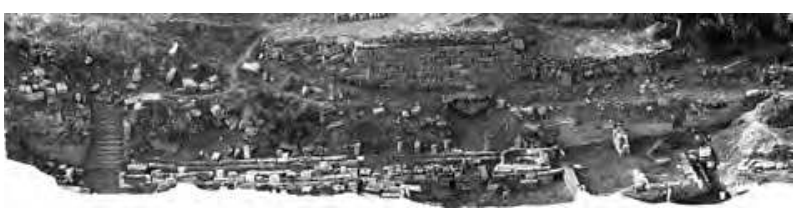

Fig. 5.1.1 : Modélisation 3D de la stoa nord avant les travaux de 2017

(Fr. Marchand-Beaulieu).

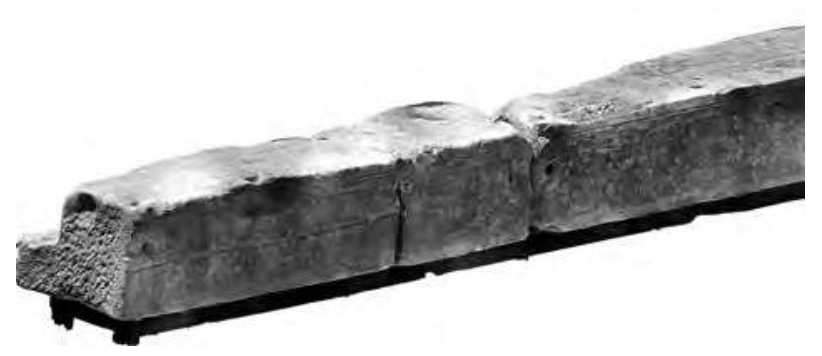

Fig. 5.1.3 : L'extrémité sud de la dédicace des Oikoi par Idrieus (Fr. Marchand-Beaulieu).

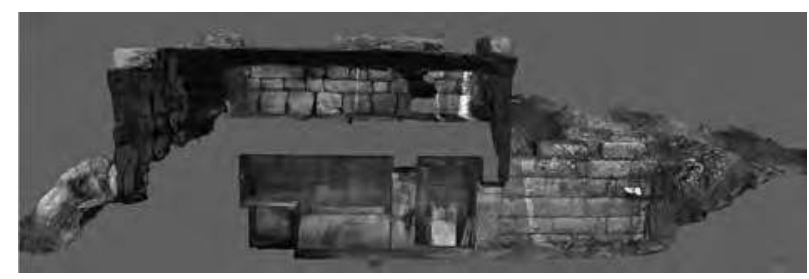

Fig. 5.1.4 : Section longitudinale de la tombe monumentale (Fr. Marchand-Beaulieu).

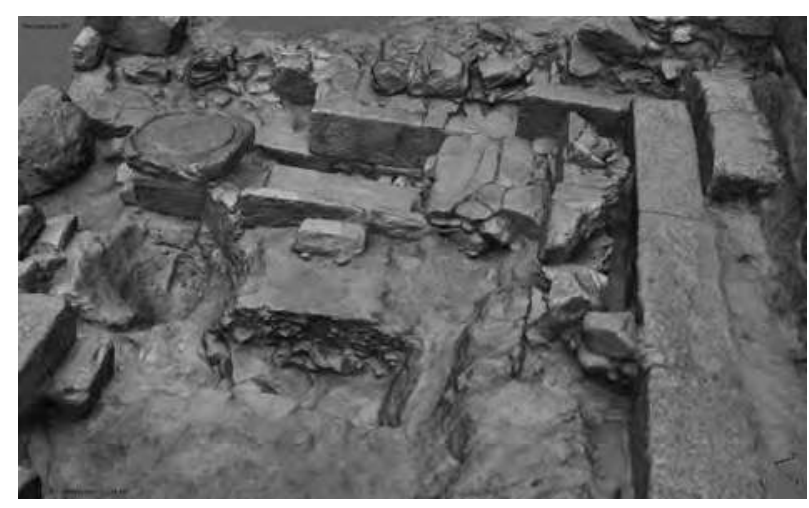

Fig. 5.1.6 : Modélisation photogrammétrique du sondage BTC Zone 1 (Fr. Marchand-Beaulieu).

Fig. 5.1.7: Photogrammétrie des vestiges du bassin de la maison dorique (nord vers le haut) (Fr. Marchand-Beaulieu).

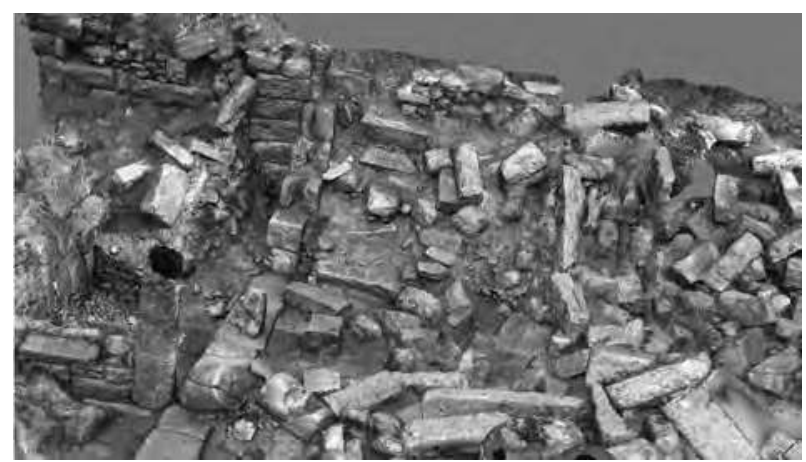

Fig. 5.1.2 : Photogrammétrie au sol de la partie nord de la stoa est (Fr. Marchand-Beaulieu).

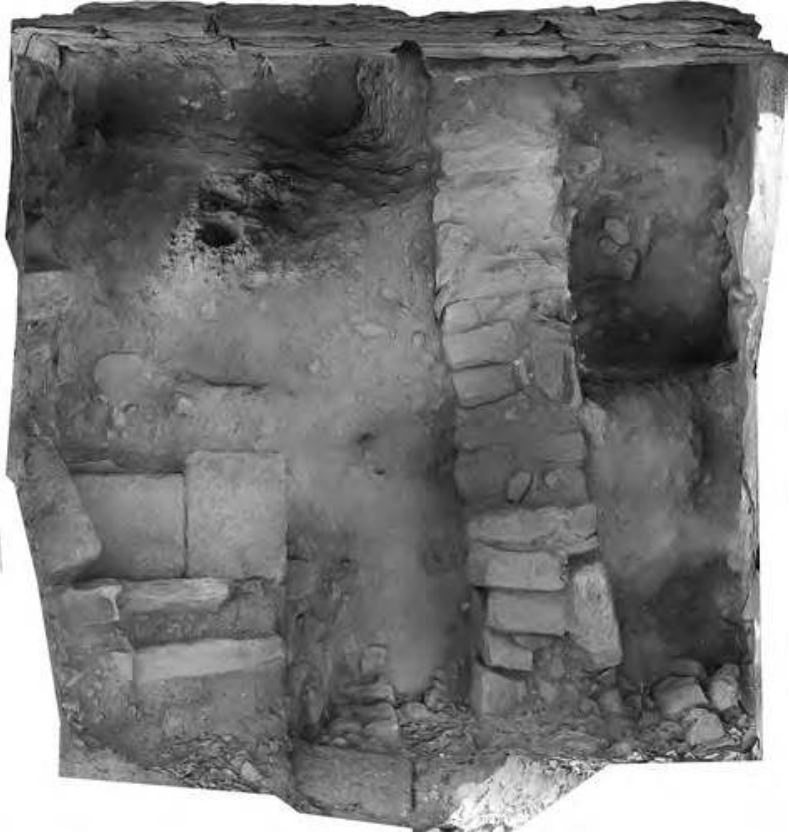

Fig. 5.1.5 : Orthophotographie du sondage S17 (Fr. Marchand-Beaulieu).

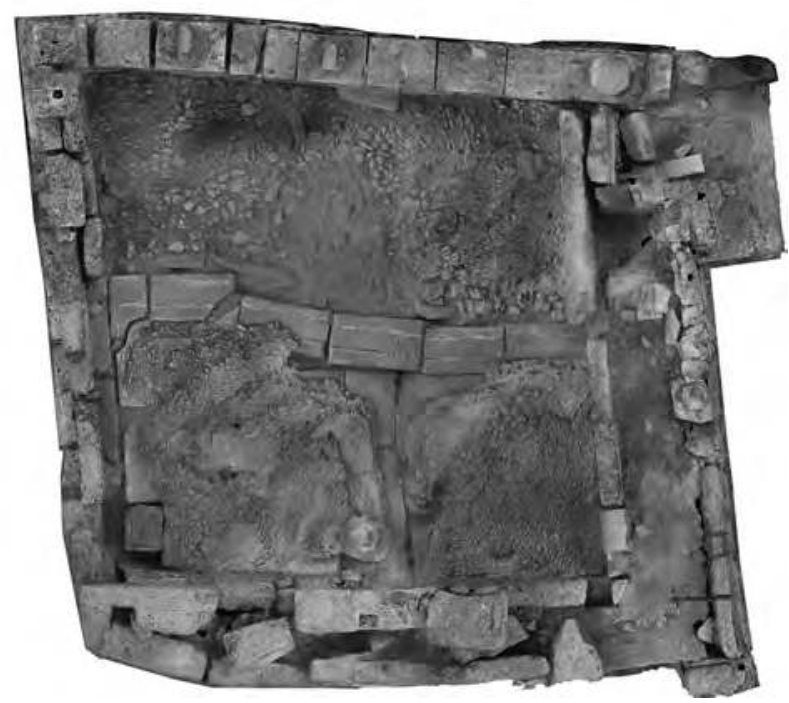




\subsection{TEMPLE TERRACE}

(by R. Hedlund and J. Blid)

This year was the fifth field campaign in a research project focusing on the Hellenistic and Roman buildings surrounding the Temple Terrace at Labraunda. A number of studies have treated finds from this period, most notably the works of Crampa, Gunter and Liljenstolpe \& von Schmalensee $^{67}$. However, no attempt at a synthesis has been made. In this project, a number of buildings are currently studied. Preliminary investigations of the preserved architectural remains were done during the field campaign in $2011^{68}$. In 2012, we initiated a more systematic architectural survey of the Roman remains of the Temple Terrace, and its significance for the layout and functions of the sanctuary ${ }^{69}$.

In the first three campaigns, we focused mainly on the architectural remains that we attributed to the so-called Terrace $\mathrm{M}$ (the terrace below the Temple Terrace). This represents one of the most monumental buildings in Labraunda dating from the Roman period. The building has to date not been thoroughly excavated; however, some conclusions can be drawn from various earlier documentations. Drawing on studies of the architectural members preserved from the M Building, and the layout of the terrace where this building stood, we have previously proposed an architectural layout in the shape of a multi-storeyed covered hall. Similar halls are known from several other sites in SW Asia Minor. The function of this building is still unclear; however, a limited excavation allowed us to reconstruct its construction and building phases with a high level of accuracy.

In 2014 we expanded the study to include other Hellenistic and Roman buildings on the eastern side of the Temple Terrace/Terrace B and the Terrace Y (east of the Temple Terrace). The study included two buildings: the Y Building, which served as a monumental entrance to the Temple Terrace, and the B/Y Stoa which served as a link between the propylon and the M Building.

The aim of this year's campaign was twofold: 1 . complete the documentation of architectural structures and members and 2. provide more detailed reconstructions for these buildings.

The campaign yielded a large number of drawings: a total of 6 reconstructions, 5 elevations, 1 updated plan, 2 find plans and 130 detailed draw- ings of architectural members. The block drawings comprise the following categories of blocks: 30 cornice blocks; 24 architraves; 14 anta blocks, 7 stylobate blocks; 2 columns; 3 entablature blocks; 8 door blocks for the triple doors in the Y Building; and one block with an uncertain function (probably from the NE corner of the east facade of the Y Building).

In addition to this, one previously unrecorded anta-block was documented.

As the documentation is now finished, we can propose functions of the buildings. We have therefore chosen to change their names, in order to better distinguish their functions:
1) Temple Propylon (Y Building)
2) Propylon Stoa (Y Stoa)
3) Well House Stoa (M Building)
4) Connecting Stoa (B/Y Stoa)

This season we were able to reconstruct the appearance of the Temple Propylon, which lead to the temple area, with a much higher level of accuracy than before. One important discovery is that the antae of this building had alternating bonds; this is indicated by a cut offset on gneiss blocks corresponding to bonded anta-blocks. Moreover, pry-holes on two anta-blocks (B 62 and B 79) indicate that narrower blocks were put on top with crow-bars. Moreover, the new plan with documentations of pry-holes and clamps enable reconstruction of wall shifts now gone.

We now also have a fuller understanding of the entablature of the east side of the Temple Propylon, which means that the roof structure can also be reconstructed. As the pediment/tympanon blocks have been studied, a total height of this façade can be calculated. A more exact reconstruction of the triple doors, including thresholds, in the middle of the building has also been made. Accordingly, the east façade of this building can also be reconstructed with high probability.

The western façade can also be reconstructed with some probability. This is not least because of studies of older documentation which led to the discovery of a marble stylobate which has been removed since the original excavations. This could be matched with two corner-blocks from a pediment documented during earlier seasons. Thus, with some probability the Temple Propylon featured a monumental façade, facing the Temple of Zeus in the Roman period. The western façade also featured

67) I.e Blid 2016; Crampa 1972; Gunter 1995; Liljenstolpe and von Schmalensee 1996.

68) Karlsson et al. 2012: 85.

69) Henry et al. 2013: 327-336. 
two returning antae. The NW anta-blocks (i.e. the left side of the façade, seen from the terrace) featured a rougher facing surface on the exterior only finished towards the right edge; this is possibly because this side was partly hidden behind the exedra and North Stoa.

The reconstruction of the Well House Stoa is still uncertain in a number of respects. It is possible that the so-called Connecting Stoa was built as an extension of the Well House Stoa, rather than as a separate building, which leads to a number of suggestions regarding the movement on the Temple Terrace. We also have a clearer idea concerning technical features of the entablature such as lewisand clamp holes.

The inscriptions on the architrave-blocks (I.24a-b) have also been studied further. The fragment of one previously unknown architrave block (Y 2011-1), discovered on the Y Terrace during the 2011 campaign, must have been a right corner block and cannot be placed in any of the structures that have been excavated until now. Possibly, it was part of the Propylon Stoa, which continues to the east from the NE corner of the Temple propylon and remains unexcavated.

The studies that have now been concluded involve the following chronological periodization:

- Archaic/pre-Hekatomnid period: the Temple Propylon is built.

- Late Classical to Early Hellenistic period: The Well House is built and the Temple Propylon is rebuilt.

- Hellenistic age $\left(3^{\text {rd }} / 2^{\text {nd }} \mathrm{c} . \mathrm{BC}\right)$ : a stoa is built on the Well House Terrace.

- Early $2^{\text {nd }}$ c. AD: the North Stoa (dedicated to Trajan) and the Temple Propylon rebuilt.

- Early/mid $2^{\text {nd }} \mathrm{c}$. AD: another storey added to the Well House Stoa. The new Well House Stoa has a monumental marble façade towards the Temple Terrace. The Connecting stoa is constructed.

- Late $3^{\text {rd }} /$ early $4^{\text {th }}$ c. AD: the Well House Stoa has collapsed due to poor construction and been replaced by what seems to be a domestic area. The Connecting Stoa is probably dismantled and some of the anta-blocks are re-used on the Temple Terrace.

- $4^{\text {th }}$ c. AD: the Temple Propylon is possibly repaired, the Temple Terrace is kept in provisional shape, but the other monumental buildings have collapsed.

\subsection{ETUDES CERAMIQUES}

(par V. Lungu)

Le programme d'étude des céramiques de Labraunda, prévu entre 15 juin - 15 août 2017 , concernait surtout les céramiques de plusieurs ensembles issus des fouilles des années précédentes, ainsi que de celles de deux secteurs fouillés cette année (Bains Est et Bassin Romain). Notre travail a ciblé les mobiliers funéraires de la nécropole, les divers contextes de l'Andrôn A, de l'Acropole et du Bâtiment en Pi au pied de la tombe monumentale ${ }^{70}$.

Au cours de la saison 2017, nous avons étudié plusieurs milliers de tessons céramiques. Parmi ceux-ci, on a eu la possibilité d'en identifier, restaurer, photographier, dessiner et étudier quelques centaines.

Grâce à la restauration complète ou partielle de plusieurs vaisselles céramiques, nous avons obtenu un nombre de 19 formes différentes, réparties sur toutes les époques identifiées.

Les autres objets triés indiquent des parties de vases reconnaissables, importantes pour établir la chronologie de chaque contexte, l'origine des vases importés et les caractéristiques de quelques groupes attribués hypothétiquement à des productions régionales ou locorégionales. Cette approche est particulièrement importante pour définir le faciès céramique de Labraunda.

Dans un premier temps, nous ${ }^{71}$ avons a réalisé des tableaux avec les comptages des objets découverts par secteur et par contexte, dont on donne ici un exemple (tableau I).

70) Dans le texte qui suit nous utiliserons les abréviations suivantes : Abréviations des unités fouillées : CWO = Corridor with the oikoi, SSB = Sous-sol Byzantin ; Abréviations des groupes céramiques : LRC $=$ Late Roman Pottery C ; Appellation des formes : Am = amphore, $\mathrm{BA}=$ bol attique, $\mathrm{BAD}=$ bol avec anses doubles , $\mathrm{BB} 2=$ bol imité d'après le type $\mathrm{ESB} 2 \mathrm{Forme} 80, \mathrm{BCPh}=\mathrm{bol}$ de la classe phocéenne, $\mathrm{BC}=$ bol de type cnidien, $\mathrm{BCW}=$ bol type "Campana Ware", $\mathrm{BLH}=$ bol à lèvre à l'horizontale, $\mathrm{BR}=$ bol à rosettes, $\mathrm{BSC}$ $=$ bol $\mathrm{ESC}, \mathrm{BSI}=$ bol de la sigillata italique, $\mathrm{CB}=\mathrm{Coupe}$ biconique à deux anses, $\mathrm{CC}=$ cruche type "collarino", $\mathrm{CO}=\mathrm{coupe}, \mathrm{CPh}=$ cruche de la classe phocéenne, $\mathrm{CsC}=$ casserole type $\mathrm{C}, \mathrm{Cv}=$ couvercle, $\mathrm{J}=$ jatte, $\mathrm{L}=$ lampe, $\mathrm{Ol}=$ olla, $\mathrm{PC}=$ pot de cuisine, $\mathrm{Pl}=$ plat, $\mathrm{PlCW}=$ plat de type "Campana Ware", $\mathrm{PP}=$ plat à poisson, $\mathrm{PF}=$ plat à feu, $\mathrm{S}=$ salière, $\mathrm{T}=$ turibullum, $\mathrm{U}=$ unguentarium, $\mathrm{VC}=\mathrm{vase}$ à collerette.

71) Vasilica Lungu, responsable du département "Céramique"; membres de l'équipe en 2017 : Alina Muşat Streinu, Cristina Georgescu, Alina Ciobotaru, Liviu Iancu, Eloise Frebault, Gogla Durak, Merve Gura. 
Tableau I : Secteur des Bains Est.

\begin{tabular}{|c|c|c|c|}
\hline Pièce 2 & $\begin{array}{l}\text { Sélectionnés } \\
\text { Non-sélectionnés }\end{array}$ & $\begin{array}{r}182 \\
17\end{array}$ & Total : 199 \\
\hline Pièce 5 & $\begin{array}{l}\text { Sélectionnés } \\
\text { Non-sélectionnés }\end{array}$ & \begin{tabular}{|l|}
147 \\
659 \\
\end{tabular} & Total : 706 \\
\hline Pièce 7 & $\begin{array}{l}\text { Sélectionnés } \\
\text { Non-sélectionnés }\end{array}$ & $\begin{array}{r}41 \\
195\end{array}$ & Total : 236 \\
\hline Pièce 8 & $\begin{array}{l}\text { Sélectionnés } \\
\text { Non-sélectionnés }\end{array}$ & $\begin{array}{l}124 \\
355\end{array}$ & Total : 459 \\
\hline Area 10 & $\begin{array}{l}\text { Céramique } \\
\text { Marbre } \\
\text { Verre }\end{array}$ & $\begin{array}{r}170 \\
12 \\
6 \\
\end{array}$ & Total : 188 \\
\hline 5 contextes & & & Total général: 1.788 \\
\hline
\end{tabular}

Après le tri du matériel, les pièces sélectionnées ont été partiellement photographiées et dessinées (tableau II).

Tableau II : Total des dessins réalisés par secteur.

\begin{tabular}{|l|c|c|c|c|c|c|}
\hline Secteurs & Acropole & Andrôn A & Built tomb & East Bath & Water Pool & Total \\
\hline No dessins & 115 & 50 & 229 & 69 & 29 & 492 \\
\hline
\end{tabular}

Nous avons aussi réalisé des listes provisoires du matériel par époque, pour chaque secteur, comme le montre le tableau suivant (tableau III).

Tableau III : Le secteur des Bains Est (sondage 2017).

Liste des US avec le matériel céramique préromain.

\begin{tabular}{|c|c|c|c|}
\hline Nr US & Céramique & Date & Observation \\
\hline $\begin{array}{l}10.006 \\
\text { zone } 10\end{array}$ & $\begin{array}{l}1 \text { assiette à vernis noir } \\
1 \text { couvercle à vernis noir } \\
1 \text { bol à décor en relief }\end{array}$ & Fin du $3^{\mathrm{e}}-2^{\mathrm{e}}$ s. av. J.-C. & fragments \\
\hline 10.008 & $\begin{array}{l}1 \text { timbre d'amphore rhodienne } \\
2 \text { bols à décor en relief }\end{array}$ & $2^{\mathrm{e}}$ s. av. J.C. & fragments \\
\hline $\begin{array}{l}10.012 \\
\text { Zone } 10\end{array}$ & $\begin{array}{l}1 \text { plat à poisson } \\
1 \text { bol à décor en relief }\end{array}$ & $2^{\text {e }}$ s. av. J.C. & fragments \\
\hline 10.014 & $\begin{array}{l}1 \text { bol à vernis noir } \\
1 \text { fragment couvercle ; engobe rouge } \\
1 \text { bord d'amphore } \\
1 \text { bord de vase de stockage }\end{array}$ & $3^{\text {e }}$ s. av. J.-C. & fragments \\
\hline 10.021 & $\begin{array}{l}1 \text { bol à bord rainuré } \\
1 \text { bol à paroi caréné/couvercle }\end{array}$ & $2^{\mathrm{e}}$ s. av. J.C. & fragments \\
\hline 10.029 & $\begin{array}{l}1 \text { fond bolsal attique à vernis noir décor à la } \\
\text { roulette } \\
1 \text { plat à poisson attique } \\
1 \text { bol à bord incurvé ; vernis noir non-attique } \\
1 \text { bol à engobe rouge }\end{array}$ & $2 / 24^{e}$ s. av. J.-C. & fragments \\
\hline
\end{tabular}




\begin{tabular}{|c|c|c|c|}
\hline $\begin{array}{l}10.030 \\
\text { Zone } 10 \\
\text { (1er sac) }\end{array}$ & $\begin{array}{l}1 \text { lèvre de cruche } \\
1 \text { lèvre bol à bord incurvé } \\
1 \text { panse attique à vernis noir } \\
2 \text { panses à engobe noir incertains }\end{array}$ & mixte $: 4^{\mathrm{e}}$ et $2^{\mathrm{e}}-1^{\mathrm{er}}$ av. J.-C. & fragments \\
\hline $\begin{array}{l}10.030 \\
\text { Zone } 10 \\
\text { (2e sac) }\end{array}$ & $\begin{array}{l}1 \text { fragment bol ionien (Milet Villard B2) } \\
1 \text { anse bifide d'amphore de transport } \\
1 \text { épaule de cruche ou d'amphore de table à } \\
\text { bande du vernis noir } \\
1 \text { bol à vernis noir non-attique } \\
1 \text { couvercle }\end{array}$ & $\begin{array}{l}\text { fin du } 5^{\text {e }} \text { av. J.-C. } \\
\text { et } \\
2 / 24^{\text {e }} \text { s. av. J.-C. }\end{array}$ & $\begin{array}{l}\text { fragments ; } \\
\text { pour le vase à anse } \\
\text { bifide, il est de la même } \\
\text { pate qu'un fragment de } \\
\text { cruche de LAB } 16 \mathrm{~EB}, \\
\text { US } 8035\end{array}$ \\
\hline $\begin{array}{l}10.032 \\
\text { Zone } 10\end{array}$ & 1 fond de bol & $3^{\mathrm{e}}-2^{\mathrm{e}}$ av. J.-C. & fragment \\
\hline $\begin{array}{l}10.033 \\
\text { Zone } 10\end{array}$ & $\begin{array}{l}1 \text { panse de petit vase ouvert à vernis noir mat } \\
1 \text { fond grand vase fermé (amphore de table ou } \\
\text { grande cruche?) }\end{array}$ & $\begin{array}{l}\text { Incertain. } \\
\text { Il semble plutôt de la fin } \\
\text { d'ép. archaïque }\end{array}$ & \\
\hline \multicolumn{4}{|l|}{ TOTAL } \\
\hline 9 US & 31 objets & $\begin{array}{l}2 \text { périodes chronologiques } \\
\text { représentées : } \\
\text { archaïque et hellénistique }\end{array}$ & $\begin{array}{l}\text { Matériel très } \\
\text { fragmentaire }\end{array}$ \\
\hline
\end{tabular}

Une fiche a également été réalisée pour chacune des tombes de la nécropole. Ces fiches contiennent les caractéristiques principales des vases analysés et la chronologie. Au total, 76 fiches ont été complétées (tableau IV).

Tableau IV : Exemple de fiche par tombe (76 au total) : ici la tombe 78: $3 / 44^{\mathrm{e}} \mathrm{s}$. av. J.-C.

\begin{tabular}{|c|c|c|c|c|c|c|c|}
\hline No. & Forme & Marquage & $\begin{array}{l}\mathbf{N}^{0} \\
\text { pièces }\end{array}$ & Pâte & Surface & Date & Analogies \\
\hline 1. & Bolsal & LAB08.T78-02 & 1 & $10 \mathrm{YR} 6 / 4$ & $\begin{array}{l}\text { Engobe } 10 \mathrm{R} \\
5 / 8 \text { rouge }\end{array}$ & $\begin{array}{l}\text { 3/4 } 4^{\mathrm{e}} \text { s. av. } \\
\text { J.-C. }\end{array}$ & \\
\hline 2. & Amphoriskos & LAB08.T78-01 & 1 & $\begin{array}{l}\text { Gley } 2,5 \text { Y 4/2 int. } \\
5 \text { YR 5/6 ext. }\end{array}$ & - & $\begin{array}{l}\text { 3/4 } 4^{\mathrm{e}} \text { s. av. } \\
\text { J.-C. }\end{array}$ & \\
\hline 3. & Olpe & LAB08.T78-03 & 1 & $\begin{array}{l}5 \text { YR 6/4 int. } \\
7.5 \text { YR 5/6 ext. }\end{array}$ & - & $\begin{array}{l}\text { 3/4 } 4^{\mathrm{e}} \text { s. av. } \\
\text { J.-C. }\end{array}$ & \\
\hline
\end{tabular}

L'évaluation quantitative de chaque catégorie céramique identifiée par secteur a été présentée dans des tableaux statistiques, d'après le modèle suivant, en réalisant environ 89 fiches statistiques (tableau V).

Tableau V : Secteur East Bath 2017, Sondage "Basilica".

\begin{tabular}{|l|c|c|c|c|}
\hline Catégories/classes & No fragments & MNV & $\begin{array}{c}\text { Pourcentage } \\
\text { de total }\end{array}$ & $\begin{array}{c}\text { Pourcentage } \\
\text { de MNV }\end{array}$ \\
\hline Amphores & 37 & 34 & $13,55 \%$ & $15,45 \%$ \\
\hline Céramique fine & 78 & 69 & $28,57 \%$ & $31,36 \%$ \\
\hline Céramique commune & 27 & 24 & $9,89 \%$ & $10,91 \%$ \\
\hline $\begin{array}{l}\text { Céramique de } \\
\text { cuisine }\end{array}$ & 110 & 93 & $40,29 \%$ & $42,27 \%$ \\
\hline Autres & 18 & & $6,59 \%$ & \\
\hline Non-déterminé & 31 & & $11,36 \%$ & \\
\hline
\end{tabular}


De toute la masse céramique étudiée, on doit noter la faible présence des céramiques communes et fine, à pâte grise. Elles représentent à peine 1,5\% de l'ensemble. Il est probable qu'elles soient des importations, quelques tessons étant inexploitables à cause de leur état de fragmentation. Il semble que la pratique des vases à pâte grise n'était pas répandue dans la zone de notre site. Cette absence n'est pas compensée non plus par des importations en grandes quantités de produits d'autres provenances. En règle générale, les quotas de céramiques communes importées sont modestes.

A coté de divers centres d'importation, identifiés en étudiant les caractéristiques de la pâte et le traitement de surface, nous avons repéré, pour quelques-uns de ces vases, des caractéristiques morphologiques attribuables plutôt à des centres locorégionaux. Une attention particulière a été portée au répertoire des formes de chacun des groupes distingués par fonctionnalité des vases.

L'analyse chronologique du matériel nous a conduit à obtenir de nombreuses informations sur les époques suivantes : archaïque, hékatomnide, hellénistique, romaine, byzantine.

Une partie du matériel traité provient du secteur Andrôn A et dont quelques résultats sont présentés dans les lignes suivantes ${ }^{72}$ :

L'Andrôn A a été fouillé pendant plusieurs campagnes à partir de 1948 jusqu'en 2016. Malheureusement, les publications des fouilleurs n'offrent que peu d'informations relatives aux contextes fouillés avant 2014. Les céramiques étudiées proviennent des trois dernières campagnes de 2014-2016. Toutes les céramiques de chaque unité stratigraphique fouillée au cours de ces campagnes ont été prises en compte. L'intégralité des fragments, typiques et atypiques, a été étudié, sélectionné et comptabilisé ; les fragments reconnaissables ont été répartis en catégories bien définies. Pour compléter le corpus référentiel local, commencé il y a trois ans, nous avons considéré nécessaire de dessiner systématiquement tous les individus inventoriés par unité stratigraphique.

On dispose pour l'instant d'une base référentielle d'environ 476 objets inventoriés sur un total de 3.385 objets provenant de ce secteur, dont 367 identifiés servent comme repères chronologiques dans leurs contextes stratigraphiques. En effet, nous avons constaté un déséquilibre important entre le nombre de fragments significatifs (anses, bords, bases, parties de corps typiques...) et les fragments atypiques. Ces derniers ont été prélevés, comptés et conservés hors du dépôt, qui est réservé seulement aux parties sélectionnées.

A partir de cette documentation assez abondante, nous avons obtenu une classification des céramiques fondée sur des critères technologiques et morphologiques. Elle nous offre la possibilité de juger la fréquence de chaque type et de chaque variante dans les contextes de l'Andrôn A, de déterminer la datation et l'origine, de constater le caractère résiduel de telle ou telle pièce afin de définir des assemblages caractéristiques pour chaque unité fouillée. De cette façon, on arrive à obtenir le faciès général des céramiques liées à ce monument, associant les productions locales aux produits importés.

Les analogies avec les céramiques issues des grands sites égéens ou méditerranéens sont nombreuses et difficilement présentables ici dans ces quelques lignes de rapport. Mais encore, ces données doivent être utilisées avec beaucoup de prudence en tenant compte du fait que l'étude de ce mobilier se heurte encore à l'absence de références locales fiables.

Pour donner quelques repères chronologiques sur les niveaux d'occupation de ce monument, on présente ici par période historique une petite sélection de formes identifiées.

\subsubsection{REPARTITION DES MOBILIERS CERAMIQUE PAR PERIODE HISTORIQUE}

\section{- Epoque hékatomnide}

Quelques pièces de céramiques fines de la fin de l'époque classique et hellénistique retrouvées dans les Pit 2, Pit 3, Pit 5, Pit 7, Pit 17, apparaissent comme des fragments résiduels. Elles sont aussi les plus anciennes céramiques fines de l'Andrôn A. Les formes attestées sont : salière attique à parois concave datée de 350-325 av. J.-C. ${ }^{73}$, salière attique à bord incurvée de la même période ${ }^{74}$ et bolsal atti$\mathrm{que}^{75}$, contemporain des deux formes précédentes. En effet, le répertoire apparaît très réduit ainsi que les nombres des exemplaires identifiés. On note toutefois l'absence d'autres importations de céramiques de table de la même période attestées par d'autres fouilles. Le nombre des produits classés comme locorégionaux est aussi fort réduit : un bol-

72) L'étude général du matériel sera présenté dans une étude à part.

73) Agora XII : 302, no 937, pl. 34.

74) Agora XII : 303, no 949, pl. 34.

75) Agora XII : 275, $\mathrm{n}^{\circ} 361$. 

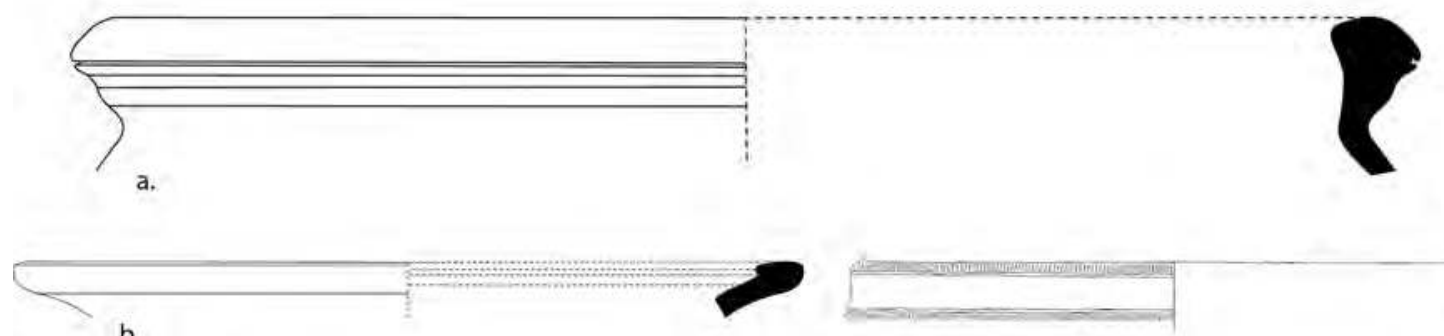

b.

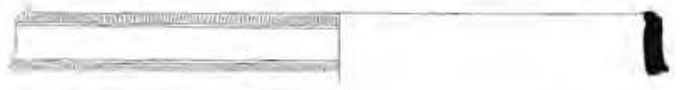

c.
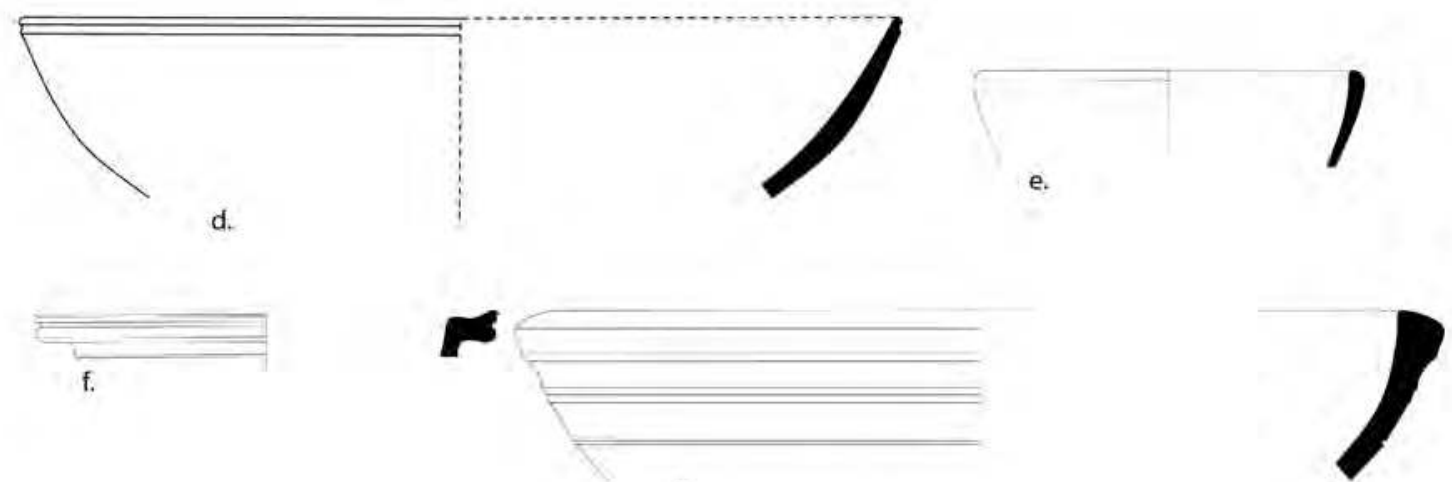

g.

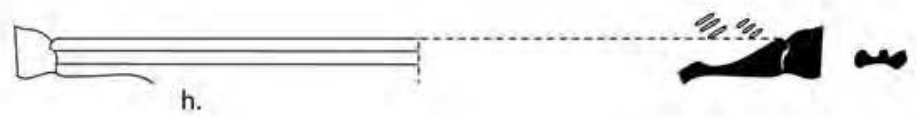

1

j.

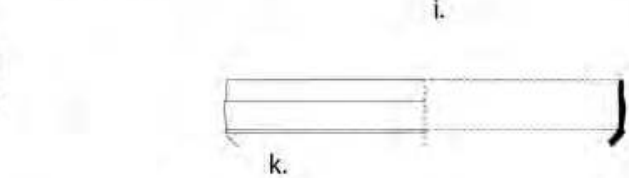

k.
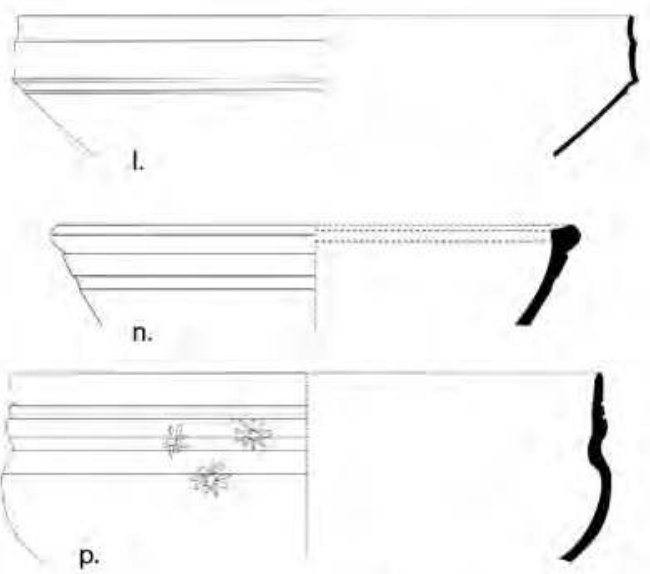

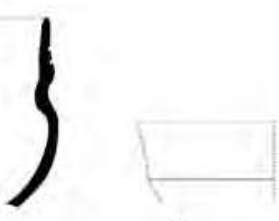

q.

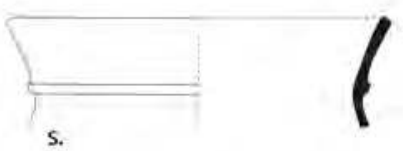

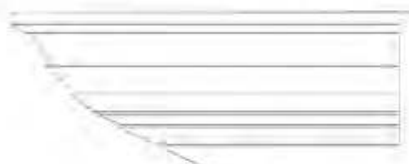

m.

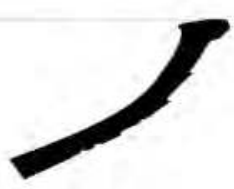

1

o.

$\int$

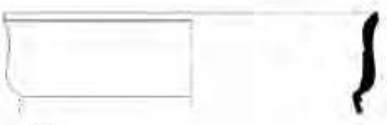

r.

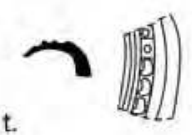

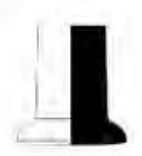

u.

Fig. 5.3.1 : Echantillons des céramiques analysées en 2017 (échelle 1/2) : a. PP01, b. PP02, c. BSI 01, d. BSI 02, e. BSC 01, f. B74 01, g. BCPh 01, h. BAD 01, i. CB 01, j. BC 04, k. BC01, l. BC 05, 1.BC 05, m. BB2 01, n. BB2 02, o. BR 01, p. BR 02, q. CO 01, r. CPh 01, s. CC 01, t. L 02, u. U 01 (V. Lungu). 
sal fragmentaire en pâte locale/régionale et couverte d'engobe rouge de Pit 7 (sans illustration) est contemporain de pièces attiques rapportées ${ }^{76}$.

\section{- Epoque hellénistique}

Nous avons identifié dans le Pit 4 une forme de petit plat ou un plat à poisson à bord tombant de petite taille (Fig. 5.3.1.a et b), datable de 150-50 av. J.-C. La forme PP 01 est reconnue en plusieurs variantes d'époque hellénistique dans d'autres secteurs ${ }^{77}$, tandis que la deuxième (PP 02) est plutôt $\operatorname{rare}^{78}$.

\section{- Epoque romaine}

Les niveaux romains ont fourni une très importante quantité de céramiques appartenant pour la plupart à la période des $1^{\text {er }}$ s. av. - $2^{\mathrm{e}} \mathrm{s}$. ap. J.-C. et, de façon plus discrète aux siècles suivants jusqu'à l'époque byzantine.

Le comptage des objets par catégorie montre l'absolue prépondérance des céramiques du début de l'époque romaine, représentées à plus de $60 \%$ dans l'ensemble des contextes étudiés. Le reste des céramique se divise entre les autres époques attestées.

\subsubsection{SELECTION DES CATEGORIES ET FORMES REPRESENTATIVES POUR LE SITE}

Pour certaines catégories céramiques (céramiques de table, vases de stockage domestique etc.), sont utilisées des argiles fines, souvent riches en calcaire et en mica, impropres à résister au feu. Pour une partie des céramiques communes, la pâte est demi-fine et les céramiques de cuisine se caractérisent par une argile typique, qui assure la résistance du vase aux flammes du feu.

\section{a. Céramiques fines}

Le répertoire des céramiques de table ou à servir apparaît assez varié, comme le montrent les vases enregistrés. On note toutefois la faible présence des céramiques de table importées de centres de production éloignés, par rapport à la quantité plus importante des importations d'Asie Mineure et de la région vers notre site. Les céramiques fines sont utilisées, en règle générale, pour les vases à servir dont l'inventaire des formes dans sa totalité, sans être nombreux, est toutefois représentatif.

\section{a.1. Importations italiques}

Bols

L'inventaire général des bols indique une variation riche et complexe. De ceux-ci, on notera les importations italiques qui sont toutefois présentes en petit nombre : un bol (Fig. 5.3.1.c) identifié comme Atlante II, forme $47=$ Hayes Shape XVIIC de la fosse "Pit 4", date du début du $1^{\text {er }}$ s. ap. J.-C..$^{79}$; du même contexte est sorti également le bol Atlante II, forme $22 \mathrm{~A}$ (Fig. 5.3.1.d), daté ca. 50-25 av. J.-C. ${ }^{80}$

\section{a.2. Importations d'Asie Mineure}

\section{Bol}

L'inventaire de l'Andrôn A montre une variété assez réduite des bols importés d'Asie Mineure : un bol ouvert de "Post Hole" (Fig. 5.3.1.e) indique une forme d'ESC du $1^{\text {er }}$ siècle ap. J.-C., dont l'origine reste à déterminer ; la forme est présente aussi à Ephèse dans un contexte de 220-230 ap. J.-C. ${ }^{81}$. Un bol ouvert fragmentaire de la sigillée orientale de "Pit 4" (BLH 01) annonce la présence d'un "flat based dish Forme 58.B" de Hayes, datable de $c a$. 290/300-375 ${ }^{82}$. Parmi les plus récents, on trouve un bol paru dans les fouilles de "SSB" (Fig. 5.3.1.f). C'est la première fois qu'une telle forme est rapportée de Labraunda. Elle est signalée par un petit fragment du bord qui comporte des caractéristiques typologiques de la Forme 74 de Hayes, daté entre 460-475, ou autour du troisième quart du $5^{\mathrm{e}} \mathrm{s}$. ap. J.-C. ${ }^{83}$.

Un autre bol à panse conique et paroi mince ("sigillata wares") de l'unité "Floor 1" est une importation de l'ouest de l'Asie Mineure avec des analogies à $\mathrm{Troie}^{84}$ avec une chronologie du $1^{\text {er }}$ siècle ap. J.-C., éventuellement entre les années 10

76) Il a des nombreuses analogies dans les tombes de la nécropole de Labraunda et d'autres secteurs de fouilles, voir Lungu 2016 : 235, fig. 75, T61.01 et T75/01.

77) Pour l'évolution de la forme entre le $2^{\mathrm{e}}$ et le $1^{\mathrm{er}}$ siècle av. J.-C., voir l'examen des plusieurs variantes d'Ephèse fait par Landstätter 2003 : 33-34, K111-K177.

78) Le petit fragment a une proche analogie à Sardis, voir Rotroff et Oliver $2003: 30, n^{\circ}$ 67, pl. 13, "Late Hellenistic".

79) Agora XXXII : $138, n^{\text {os }} 154-158$, fig. 6.

80) Agora XXXII : 132, $\mathrm{n}^{\text {os }} 88-92$, fig. 4.

81) Landstätter $2005: 325$, K 591, pl. 184.

82) Hayes $1972: 96-97$, fig, 14.15 , ca. 290/300-375

83) Pour identification, voir Hayes $1972: 124$, fig. 21

84 ) Hayes $2000: 291$, fig. 19.2 ; et $1995: 190$, fig. 3 , $\mathrm{n}^{\text {os }} 30-31$. 
et 50/60 ap. J.-C. L'exemplaire le plus tardif est un bol LRC Forme 3 Hayes de "Floor 2" (Fig. 5.3.1.g), daté vers le milieu du $6^{\mathrm{e}} \mathrm{s}$. ap. J.-C. ${ }^{85}$ et attribué à la classe phocéenne ${ }^{86}$.

\section{Plat}

Rarement apparaissent les plats d'importation comme le BCW 01 du "Central Pit" à décor de guilloches à l'intérieur similaire avec une pièce de "Campana Ware", daté ca. 75-50 av. J.-C. ${ }^{87}$. Un autre exemplaire à décor de guilloches vers le bord provient de "Pit 4" (Fig. 5.3.1.h) ${ }^{88}$ annonce déjà le milieu du $\mathrm{II}^{\mathrm{e}}$ s. ap. J.-C. (= bol Hayes 893$)^{89}$. Il est le seul exemplaire doté de deux petites anses horizontales qui le consacre comme vase utilisé dans les pratiques rituelles et l'explication de la présence des deux anses est donnée par Hayes "perhaps for priestly use" ${ }^{\prime 00}$.

\section{Coupe biconique à deux anses}

Un petit fragment d'embouchure d'une coupe biconique à deux anses provient du "Pit 4" (Fig. 5.3.1.i) ${ }^{91}$. Fabriqué dans une pâte fine (2.5 YR 6/8), avec de rares et minuscules inclusions calcaires, il est couvert à l'intérieur et à l'extérieur d'un engobe brun (2.5 YR 4/6) à la place du vernis. Il est d'ailleurs le seul exemplaire identifié dans l'Andrôn A.

\section{a.3. Production régionale}

\section{Bol de type cnidien}

De nombreux fragments des quelques formes typiques comme les bols de type cnidien et les bols ESB2 indiquent les formes les plus fréquentes de la vaisselle à servir pour le début de l'époque romaine. Les bols de type cnidien (alias "Roman brittle ware" de Hellström ${ }^{92}$ ) attribués par nous à des productions des ateliers locaux ou régionaux ${ }^{93}$, distants de quelques kilomètres, peuvent évoluer en suivant des tendances communes, mais pas nécessairement synchroniques. Cette forme est multipliée d'une manière spécifique, autonome, et apparaît comme le principal vase à boire du début de l'époque romaine, notamment aux $1^{\text {er }}$ début du $2^{\mathrm{e}}$ s. ap. J.-C. ${ }^{94}$. Plusieurs exemplaires des unités différentes ("Pit 3", Fig. 5.3.1.j ; "Pit 4", Fig. 5.3.1.k ; BC 02 ; BC 03 ; "SSB", Fig. 5.3.1.1) reflètent la densité considérable de ces vases à boire ${ }^{95}$. Ils sont aussi apparus dans d'autres unités telles que "Pit 6", "Pit 9", "Surface 3", "Central Pit", "CWO", "Floor 1", "Floor 2" et "Surface générale".

\section{Bol de type ESB2-Atlante 80}

Une autre forme qui revient fréquemment dans les premières couches d'époque romaine est celle d'un bol que nous désignons provisoirement comme une imitation originale de la forme d'importation d'ESB2-Atlante 80 à bord angulaire et la vasque arrondie qui relient la lèvre à la base de l'épaulement ${ }^{96}$. Il a son origine dans une tradition proprement orientale. Une petite sélection des exemplaires de "Surface 1" (Fig. 5.3.1.m) et de "CWO" (Fig. 5.3.1.n) est suffisante pour montrer la variation de leur production $^{97}$. Des pièces similaires sont concentrées dans la région de Labraunda-Mylasa-Eurômos, où l'un ou plusieurs ateliers fabriquent de tels objets comparables dans un même centre - probablement près de Mylasa, d'où les produits se sont répandus dans les régions voisines.

\section{Bol à rosettes}

Deux fragments de parois de bols à décor de petites rosettes imprimées sur l'extérieur de la panse (Fig. 5.3.1.o et p) provenant de "Surface 1" et "Surface 3" indiquent un type fréquent à Labraunda, bien que leur état fragmentaire ne permette pas d'apprécier la forme complète de celui-ci. On no-

85) Hayes $2005: 24$, Beirut fig. 17, AD 550.

86) Notre exemplaires est avant la série tardive datée du milieu $6^{\mathrm{e}}$ jusqu'au début du $7^{\mathrm{e}} \mathrm{s}$. av. J.-C., voir les exemplaires plus tardifs chez Mlynarczyk 2014 : 195 : "bowl LRC Form 10A".

87) Agora XXXII : "Etruscan series", no 901, fig. 28.

88) Il est fait en pate fine (2.5 YR 7/8 light red ext et noyau gris 2.5 YR 4/1); engobe rouge (2.5 YR 5/8) à l'intérieur et à l'extérieur, et décor à la roulette sur le pourtour de la lèvre. Deux petites anses latérales au niveau de la lèvre.

89) Agora XXXII : $\mathrm{n}^{\circ}$ 893, p. 211, milieu du $2^{\mathrm{e}}$ s. ap. J.-C.

90) Proche de Hayes $2000: 294$, fig. 25.5, sans être identique.

91) Agora V : 30, G 81, pl. 4

92) Hellström $1965: 38-39, \mathrm{n}^{\text {os }} 293-313$, pls. $15,37$.

93) Çakmaklı $2016: 229$, fig. 66.

94) Agora XXXII : $\mathrm{n}^{\text {os }} 1618-1619$, fig. 51: “Cnidian B : ca. A.D. 100, mid $2^{\text {nd }}$ c. A.D.”. Pour la présence de ce bol dans d'autres secteurs de Labraunda, voir Çakmakl1 2016 : 229, fig. 66, ("Water Pool”) ; Lungu 2016 : fig. 76, T16.04 ("Nécropole").

95) Une situation pareille est mise en évidence par les statistiques sur les trouvailles de l'Eglise d'Est, "Trenches A and B", où ils sont également présents, voir Blid 2016 : p. 172, fig. 192, $\mathrm{n}^{\text {os }} 1-2$.

96) Une pièce d'importation complète a été reconstituée à la base des plusieurs fragments trouvés dans la tombe 17, voir Lungu 2016 : 242, fig. 77. Pour la forme, voir aussi Agora XXXII : 38, nos 316-320, fig. 12

97) D'autres pièces fragmentaires apparaissent dans "Pit 4", "Pit 9", "SSB" et "Surface 2". 
tera par ailleurs la spécificité particulière du nom "Fossette ware", donné par Pontus Hellström ${ }^{98}$, qui fait référence au "peculiar kind of decoration", aussi bien que la description de la forme et de ses traits morphologiques. Il propose également une datation de ces vases entre 50 et 200 ap. J.-C.

\section{Coupe}

La coupe ouverte de "SSB" (Fig. 5.3.1.q) est une forme commune à Labraunda où elle apparaît après le milieu du $1^{\text {er }}$ - début du $2^{\mathrm{e}} \mathrm{s}$. ap. J.-C. ${ }^{99}$

\section{Cruche}

Du remplissage de "Central Pit" est rapporté un fragment d'embouchure d'une petite cruche à parois fine (Fig. 5.3.1.r ; 2.5 YR 6/6 light red) dont la production a été établie au $2^{\mathrm{e}}$ s. ap. J.-C. ${ }^{100}$. Quelques exemplaires plus ou moins complets découverts à Ephèse font remonter la date vers la première moitié du $3^{\text {e }}$ s. ap. J.-C. ${ }^{101}$.

Une petite cruche à collerette $=$ "mug of type collarino" 102 de "Pit 9" (Fig. 5.3.1.s) a une chronologie plus large, entre le $1^{\text {er }}$ et le $3^{\text {e }}$ siècle ap. J.-C. A Labraunda cette pièce est une apparition isolée jusqu'à présent, contrairement à la popularité de la forme largement répandue à cette époque entre la Méditerranée et la mer Noire.

\section{Lampe}

Un sous-groupe à part, au sein du groupe de la céramique fine, est formé par quelques fragments de lampes. Un fragment sans décor de "Pit 9" (L 01) et un autre décoré de "Pit 4" (Fig. 5.3.1.t) sont parmi les meilleurs fragments conservés de cette forme. En tenant compte de leur état de fragmentation, il est difficile d'établir exactement le type, mais apparemment elles indiquent des exemplaires des $1^{\text {er }}$ et $2^{\mathrm{e}} \mathrm{s}$. ap. J.-C. D'autres fragments plus petits proviennent de "CWO" et "Pit 4".

\section{b. Céramiques communes}

Concernant les céramiques communes, on constate une relative complexité du répertoire étudié. Une observation générale est que les céramiques communes sont les plus courantes dans les niveaux romains de l'Andrôn A. Les vases des productions communes ont une pâte différente des vaisselles culinaires. Les vases à verser, comme les cruches, dominent modérément, les casseroles et les jattes ou bassins représentant une autre partie non négligeable de l'ensemble. Les amphores de transport et les lagynoi apparaissent en quantités modérées. Il faut toutefois noter que cette situation n'est pas totalement représentative de la réalité générale du site. En effet, comme nous avons affaire à des vases très fragmentés, nous ne pouvons pas toujours observer la forme complète de toutes les pièces. Toutefois, on peut noter que les vases de la céramique commune ont des pâtes différentes en indiquant des productions qui varient d'un atelier à l'autre, d'une époque à l'autre. Les formes fréquentes appartiennent aux groupes des vases à verser, de table, ou des vases de stockage domestique de différentes tailles. Beaucoup plus réduit est le nombre des petites formes cosmétiques, par exemple. Parmi ceuxci, nous avons reconnues des vases genre lécythes et unguentaria (Fig. 5.3.1.u).

\section{Vases de stockage}

\section{Casserole}

Une casserole fragmentaire (Fig. 5.3.2.a) est apparue en "SSB". Elle rappelle la forme 197 de Hayes ${ }^{103}$, datée de la fin du $2^{\mathrm{e}}-3^{\mathrm{e}}$ s. ap. J.-C. Faite d'une pâte rouge grisâtre (2.5 YR 3/1 dark redish gray), elle est couverte d'un engobe mate, dilué (2.5 YR 3/6 dark red). Il y a là possibilité d'être en présence d'un produit local.

\section{Olla}

Un vase fermé de la céramique commune trouvé dans "Pit 15", désigné par le terme olla, date du $1^{\text {er }}-2^{\text {e }}$ s. ap. J.-C. ${ }^{104}$ et il a servi probablement à stocker divers aliments sur la table ${ }^{105}$. Le répertoire de pots de stockage de taille moyenne de l'Andrôn A apparaît très varié comme le montre le fragment du bord d'un vase conçu à recevoir un couvercle de PC 02. Il est fait dans une pâte commune locale (5 YR 6/8 reddish yellow) avec inclusions calcaires et mica dense. C'est une forme locale trouvée d'habitude dans les niveaux d'époque romaine, anciens ou tardifs, des $2^{\mathrm{e}}-5^{\mathrm{e}}$ s. ap. J.-C. ${ }^{106}$.

98) Hellström $1965: 39-40, n^{\text {os }} 314-321$, p. 15 , 37, et note 6. Pour la description, voir page 39.

99) Henry et Ingvarsson-Sundström $2010: 186$, Fig. 14, Tombe T16/05.

100) Agora XXXII : 267, $n^{\text {os }} 1599-1600$, fig. 50 .

101) Landstätter $2005: 327$, K 611-614, pl. 185, contexte de 220-230 ; K 684-686, de la première moitié du $3^{\mathrm{e}} \mathrm{s}$. ap. J.-C.

102) Hayes et al. $1998: \mathrm{n}^{\circ} 20$; Agora XXXII, 102-103, 267-268, $\mathrm{n}^{\circ} 1602-1608$, figs. 50-51: “ca. A.D. 100 or early $2^{\text {nd }}$ century at least". La plus tardive est la cruche numéro 1607 , apparue dans un contexte du $3^{\mathrm{e}} \mathrm{s}$. ap. J.-C.

103) Hayes $1972: 209$, fig. 36.

104) Landstätter $2005: 312$, K 455 (Topf), pl. 177, d'un contexte du début du II ${ }^{\mathrm{e}}$ s. ap. J.-C.

105) Profile sembleable avec les vase de cuisine, voir Zabehlicky-Scheffenegger et Schneider $2005: 64$, fig. 1.1.

106) Blid $2016: 118, \mathrm{n}^{\circ} 19$, fig. 122: Late Antique LF1. 


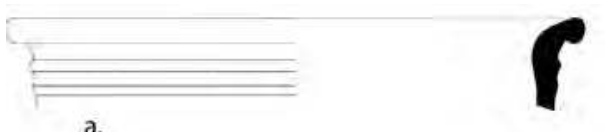

a.

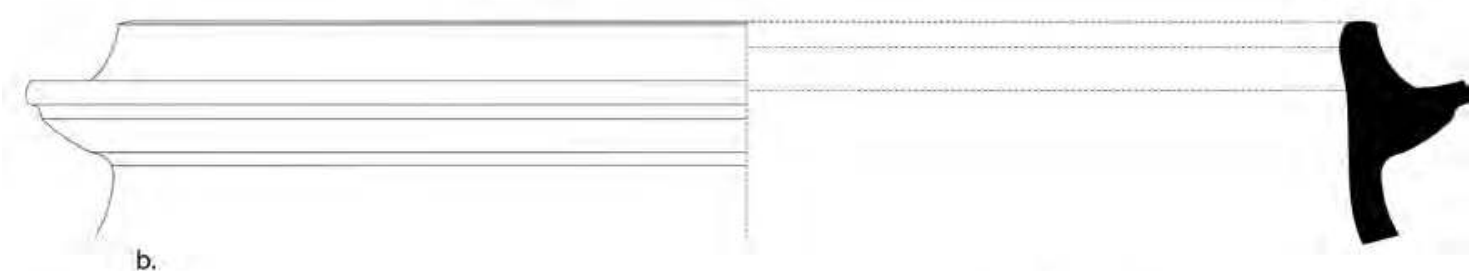

b.

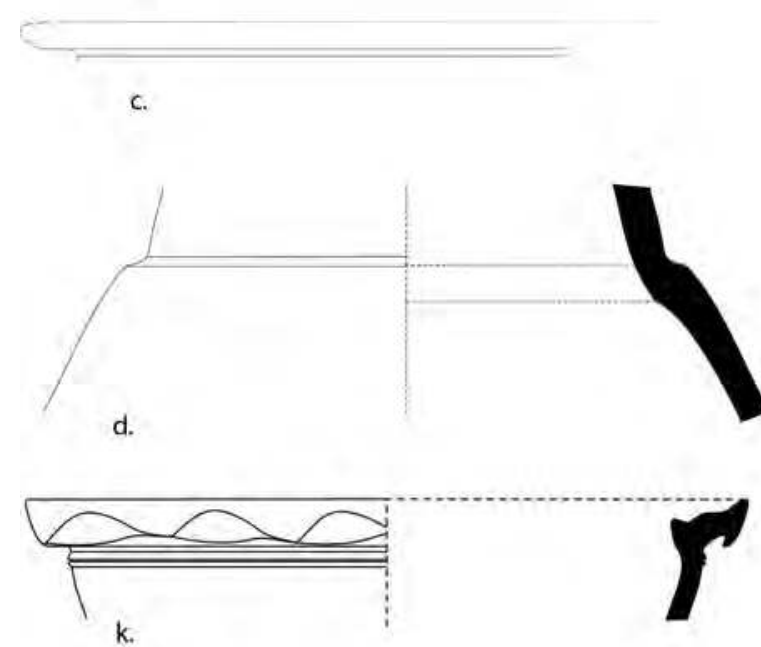

k.

e.
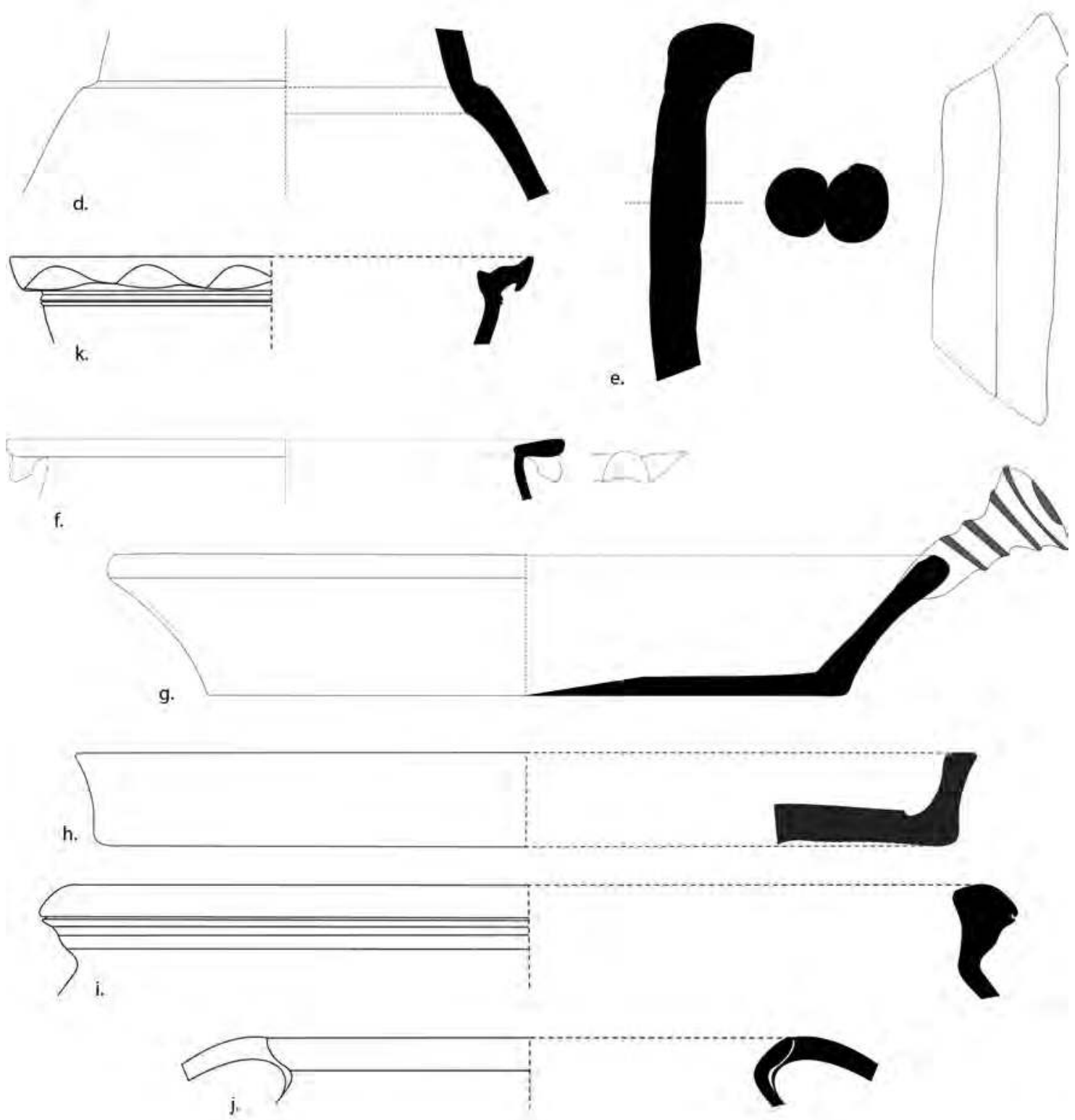

Fig. 5.3.2 : Echantillons des céramiques analysées en 2017 (échelle 1/2) : a. CsC 01, b. VC 01, c. PI 01, d. Am01a, e. Am01b, f. Ol 02, g. PF 01, h. PF 02, i. PC 01, j. PC 03, k. T 01 (V. Lungu). 
Vase à collerette

Un seul fragment du bord d'un vase à collerette a été trouvé dans "Pit 9" (Fig. 5.3.2.b). Il s'agit d'une forme bien connue à Labraunda dans les couches hellénistiques tardives $\left(3^{\mathrm{e}}-2^{\mathrm{e}} \mathrm{s}\right.$. av. J.-C.). Pour la datation de tels vases, on peut évidemment rappeler les exemplaires signalés dans les remblais de BTC ou dans le remplissage de la tour 3 de l'Acropole ${ }^{107}$, aussi bien qu'à Délos ${ }^{108}$.

\section{Pithoi}

Dans ce groupe sont ajoutés les vaisselles de grande taille, des vases à collerette et divers vases de stockage de taille moyenne avec ou sans anses. La part des pithoi de grande taille, est elle aussi, probablement plus importante que les autres vases de stockage. Cette situation est probablement due à la présence d'objets résiduels des dernières couches d'occupation datant en générale de la période romaine où des tels récipients sont d'avantage présents. Par contre, un fragment d'embouchure d'un pithos de grande taille de "Pit 8" (Fig. 5.3.2.c) était en compagnie d'un petit fragment d'anse d'un bolsal attique de 350-325 av. J.-C., une forme commune parmi les trouvailles de Labraunda ${ }^{109}$.

\section{Amphores de transport}

Le groupe des amphores de transport n'est pas nombreux, mais il est, par contre, varié. Un fragment d'anse bifide et un autre d'épaule trouvés dans CWO (figs. 5.3.2.d et e : pâte 2.5 YR 7/8 light red) sont attribués à une amphore du groupe des amphores sub-coennes ou Dressel $4^{110}$. Cet exemplaire semble avoir été produit dans les ateliers de l'île de Cos même. D'autres fragments proviennent de "Pit 4" et "Pit 8". Des amphores avec un profil semblable d'épaule apparaissent à Ephèse dans des contextes du troisième quart du $1^{\text {er }}$ siècle ap. J.-C. ${ }^{111}$.

Un seul exemplaire de l'embouchure d'une amphore avec le départ de l'anse (Am 04) en provenance de la Terrasse sud "Surface 3" peut être rapporté à la forme $2 \mathrm{~B}$ de Reynolds (AM 65) ${ }^{112}$, datée à l'époque Flavienne. Le centre de production de celle-ci est encore indéterminé.
Parmi les autres fragments amphoriques, deux fragments d'embouchure de deux exemplaires différents, illustrés par l'exemplaire Am 02, trouvés dans le même contexte de "Central Pit" ont été rapprochées typologiquement des certains exemplaires datant de 140-170 ap. J.-C. de l'Agora d'Athènes ${ }^{113}$. Le profil du bord renvoie aussi vers la Forme 11 de Reynolds attribuée probablement à la zone syrienne ${ }^{114}$. Le détail de l'embouchure est commun pour certaines amphores du Levant. Il s'agit de deux fragments similaires du groupe du Type 9 de Jiyeh Romain, l'ancien Porphyreon au Liban ${ }^{115}$, attribués à la production locale et datés de la fin de l'époque hellénistique au début de l'époque romaine. Quant à l'origine de nos exemplaires, cela reste à déterminer.

\section{c. Céramiques de cuisine}

En règle générale, les vases culinaires ont une pâte différente des productions communes non culinaires. Comme elles doivent résister au feu, elles sont faites dans des argiles réfractaires, le plus souvent sableuses. Les couleurs varient du rouge au brun, du gris au noir. La plus grande partie des céramiques de cuisine s'échange sur de faibles distances. Les formes sont rarement identifiées et publiées hors de l'aire de Labraunda ou de l'aire de diffusion régionale. Toutes ne sont pas répandues largement. Il faut ajouter que leurs formes sont en grande partie déterminées par rapport à des petits fragments du bord ou du fond. On peut parfois trouver des similitudes entre des vases provenant de sites très distants sans qu'il y ait de lien entre eux.

Concernant les céramiques culinaires, on constate une relative complexité du répertoire de l'Andrôn A de Labraunda. Les vases fermés dominent modérément, les casseroles et les plats à feu représentant une part faible de l'ensemble. On ajoute toutefois que cette observation n'est pas totalement valable pour l'ensemble du site. En plus, comme nous avons affaire à des vases fragmentaires, nous ne pouvons pas toujours être sûrs de la forme complète des vases, surtout de ceux aux formes atypiques. Quelques formes sont toutefois reconnues.

107) Lungu $2015: 375$, figs. 42-43.

108) Peignard Giros 2012 : 248-249, fig. 3; Agora XXXIII : fig. 26, $\mathrm{n}^{\circ} 162$.

109) Il semble être une trouvaille résiduelle.

110) Empereur et Picon 1989 : 225-227, fig. 2.

111) Landstätter $2010: 247$, A-K 575, pl. 95.

112) Reynolds $2013: 109$, pl. 53

113) Opaiț $2014: 21$, figs. 25-26.

114) Reynolds 2013 : Form 11, pl. 44. "Late Augustan/Tiberian to mid-third century A.D.".

115) Wicenciak $2014: 114$, fig. 19,5 Type 9 ; et $2016: 79,248-249$, pl. 49. 


\section{Olla}

La Olla est une forme fermée, indiquée par un petit fragment du bord du remplissage de "Floor 1" (Fig. 5.3.2.f). Cette exemplaire montre l'apparition d'une forme du $2^{\mathrm{e}}-3^{\mathrm{e}}$ siècle ap. J.-C., présente également à Ephèse dans un contexte du milieu du $2^{\mathrm{e}} \mathrm{s}$. ap. J.-C. ${ }^{116}$.

\section{Plats à feu}

Pour le plat à feu du secteur "CWO" de l'Andrôn A (Fig. 5.3.2.g), le prototype phocéen ${ }^{117}$ est très répandu en l'Asie Mineure ${ }^{118}$. Des objets aux formes semblables sont présents sur de nombreux sites de l'Egée et de l'espace anatolien aux époques hellénistique et romaine jusque dans l'antiquité tardive. Les exemplaires de l'Agora d'Athènes G114 et G115 sont des proches analogies qui assurent la dation de notre exemplaire entre le $1^{\text {er }}$ et le $2^{\mathrm{e}}$ siècle ap. J.-C. ${ }^{119}$. Les trouvailles d'Ephèse montrent même des exemplaires similaires de la première moitié du $3^{\text {e }}$ s. av. J.-C. ${ }^{120}$. Pour le plat du "Pit 16" (Fig. 5.3.2.h), le profil indique un exemplaire similaire de la classe phocéenne ${ }^{121}$, mais l'absence de trace de feu suggère plutôt un plat à servir le pain. Ainsi, la proportion des plats à feu est moins importante comme nous le montrent les statistiques par unité122. Ceci est probablement dû à la présence d'un nombre beaucoup plus élevé des vases profonds qui sont préférés aux plats à feu, certainement moins bien représentés. Cette situation suggère une activité plus intense visant à bouillir des grandes quantités de nourritures plutôt que de faire cuire les aliments à la poêle.

\section{Grands pots à feu piriformes}

Plus important est le nombre des grands pots à feu piriformes, à col peu marqué, à lèvre étirée vers l'extérieur en forme de gouttière, identifié dans les secteurs "SSB", "Central Pit" et "Pit 15". Un exemplaire tardif (Fig. 5.3.2.i) qui vient de "CWO" est d'époque byzantine et date du $5^{\text {e }} \mathrm{s}$. ap. J.-C. ${ }^{123}$, aussi bien que les pots PC 02 et PC 04 de "Surface 1" 124 . On doit noter que pour divers secteurs des fouilles du site ${ }^{125}$ la fréquence des vases avec le profil de PC 01, faits en pâte grossière et demi-fine et parfois couverts d'engobe rouge, est remarquable.

En nombre réduit apparaissent les petits pots globulaires à col court cylindrique, lèvre aplatie et le bas de panse scandé de cannelures caractéristiques des productions de la seconde moitié du $1^{\text {er }}$ s. av J.-C. et du $1^{\text {er }}$ s. ap. J.-C., considérés comme régionaux. Un fragment de la partie basse d'un tel vase (Fig. 5.3.2.j) vient de "Pit 4".

\section{$\underline{\text { Couvercle }}$}

La forme est enregistrée avec une présence fréquente et une large diversité qui semble indiquer une véritable particularité locale. On retrouve bien des paralléles sur de nombreux sites de la Méditerranée orientale, notamment en Asie Mineure dans des contextes variées. Par exemple, l'exemplaire de SSB (Cv 01) indique une forme banale d'Asie Mineure, utilisée normalement après 100 av. J.-C. ${ }^{126}$. La typologie des couvercles est beaucoup plus variée et indique qu'ils sont, de façon évidente, inspirés des céramiques communes de divers traditions ${ }^{127}$.

\section{Miscellanea}

Liée par la qualité de la pâte au large groupe de la céramique de cuisine, un seul fragment de turibulum $^{128}$ (Fig. 5.3.2.k) de "Pit 4" annonce la présence d'une forme particulière pour brûler l'encens pendant les pratiques cultuelles des $1^{\text {er }}$ $2^{\text {e }}$ siècles ap. J.-C. ${ }^{129}$. Le vase présente une lèvre projetée en position tombante légèrement oblique,

116) Landstätter $2005: 323$, K 570 (Topf), pl. 183.

117) Özyiğit, Ö. in KST 12-1, Ankara 1990 [1991] : 137f., fig. 9.

118) Hayes $2009: 13, n^{\circ}$ 123, fig. 6; 2000, fig. 18.4 (Mytilène). Il est considéré comme "Aegean type", voir Istenič et Schneider $2000: 345, \mathrm{n}^{\circ} 4$, fig. 5 .

119) Agora V: G114, G115, pl. 7.

120) Landstätter $2005: 329$, K 638, pl. 187, ca. 220-230 ap. J.-C.

121) Hayes $2009: 13, \mathrm{n}^{\circ} 17$, fig. 6.

122) Deux autres types sont publiés par Hellström $1965: 45$, n 387-389, pl. 19. Il remarquait aussi la quantité réduite de tels vases.

123) Doğer $2007:$ pl. VIII:d ; Turnovsky $2005: 641,645$, date $: 5^{\text {th }}$ century.

124) Doksanaltı 2010 : Type CW-1 (de Cnide), 775, fig. 1, et 770 pour la description de la forme complète.

125) Par exemple, Blid $2016: 64, \mathrm{n}^{\circ} 70$, fig. 60.

126) Hayes $2000: 288$, fig. $6, \mathrm{n}^{\circ} 3$. Il fait partie du groupe égéen, décrit par Istenič et Schneider $2000: 341$, fig. 5 , n ${ }^{\text {os }} 1-2$.

127) Leur typologie sera étudiée dans un volume dédié aux céramiques de Labraunda.

128 Liv. XXIX, 14, 13; Cic. Verr. 2, 4, 21, § 46; Liv. 29, 14, 13; Curt. 8, 9, 23; Val. Max. 3, 3, ext. 1; Aus. Idyll. 12, 104 ; Prud. Apoth. 479.

129) Hayes $2009: 11-12$, nº 87, fig. 6 "incense burner; Rome region fabric", avec la bibliographie citée pour différentes versions : Carandini 1970 : 99f., no 467-476, pl. XXVI ; Pohl 1987 : 363, n 48, fig. 138 ; Olcese et al. 2002 : pl. XXI, "incensiere tipo 1". Sur la destination cultuelle, voir Siebert $1999: 96-98$. 
dont un petit fragment de la lèvre avec le départ de la vasque préservé. Le bord est décoré avec des impressions ovalaires sur le pourtour de la partie tombante.

\section{En guise de conclusion}

De l'époque hékatomnide à l'époque byzantine, les assemblages céramiques s'adaptent aux évolutions des pratiques culinaires en fournissant de nouveaux produits qui n'appartiennent pas à la tradition locale des périodes antérieures. Ceux-ci imitent notamment des vases de tradition orientale ou égénne, comme on le verra plus loin dans le cas des bols de type cnidien ou des bols ESB2.

Malgré la fragmentation du matériel enregistré, il est possible d'observer que les pratiques culinaires et alimentaires dans l'Andrôn A étaient diversifiées. Sur ce point, on observe également que la cuisson bouillie ou étouffée dans des pots à feu est majoritaire, tandis que les préparations mijotées dans des plats à feu sont rares. Les vases à servir proviennent de deux directions : import et production régionale.

Les bols de type cnidien proviennent vraisemblablement du même atelier et répondent aux mêmes traditions techniques. On note simplement une préférence accrue pour la maigreur de la paroi des différents exemplaires comparables du début d'époque romaine, et cette impression est fondée sur l'observation de quelques bols fragmentaires montrant des variantes très proches. Bien que nous ignorions pour le moment l'endroit exacte de leurs production, nous définissons hypothétiquement cette catégorie comme "régionale", en tenant compte d'objets semblables trouvés à Milas et à Eurômos ${ }^{130}$. Ils apparaissent comme des marchés identiques, étroitement liés à un atelier ou un groupe d'ateliers qui exercent un quasi-monopole sur l'approvisionnement des sites alentours en couvrant les besoins des populations locales en vases à boire. On peu avancer même l'idee qu'ils étaient la forme à boire préférée dans les services de table du début de l'époque romaine. Dans une situation similaire, on trouve les quelques bols d'imitation des sigillés ESB2 qui possèdent une pâte fine, cuite en atmosphère oxydante.

Par ces observations sommaires, le répertoire de Labraunda continue de s'enrichir au fur et à mesure que les trouvailles anciennes et plus récentes sont étudiées.

\subsection{FAUNA (par I. Stojanovitch)}

Zooarchaeological research that started for the first time at Labraunda in 2016 continued in 2017. During this campaign, animal remains from the Water Pool (2015/2017), East Bath (2017) and North Stoa (2017) were recorded and studied.

Taxonomic identification was done at the site, with the aid of zooarchaeological manuals and published papers. Taxa frequency is presented by two most common quantification methods -NISP (Number of Identified Specimens) and MNI (Minimum Number of Individuals), where applicable.

Taphonomic analyses included recording traces of weathering, gnawing, burning and butchering. In the database, specimens with traces resulting from butchery were described in detail, including the type and location of the marks. Slaughtering ages for domestics were calculated according to tooth eruption and wear data.

Having in mind different types and chronological range of investigated context, the data will be presented by contexts, not as one assemblage.

\subsubsection{WATER POOL}

Kiln, $3^{\text {rd }}$ c. AD

During the excavations of the Water Pool, a Kiln dated to $3^{\text {rd }}$ century AD was uncovered and investigated $^{131}$. The faunal material from this context was collected and analyzed separately.

In general, the material is well preserved, but highly fragmented. Remains of sheep and goat dominate the assemblage $(72.5 \%)$, followed by domestic pig $(18.7 \%)$ and cattle $(8.8 \%)$ (Table 1: Fig. 5.4.1). No other domestic and/or wild species were identified in the sample.

\begin{tabular}{|l|r|r|c|}
\hline Species/Taxon & NISP & NISP\% & MNI \\
\hline Bos taurus (cattle) & 15 & 8,8 & 1 \\
\hline Sus domesticus (domestic pig) & 29 & 17,0 & 3 \\
\hline Sus sp. (pig indet.) & 3 & 1,7 & \\
\hline Ovis aries (sheep) & 6 & 3,5 & \\
\hline Capra hircus (goat) & 6 & 3,5 & \\
\hline Ovis/Capra (sheep/goat) & 112 & 65,5 & 4 \\
\hline Total identified & $\mathbf{1 7 1}$ & $\mathbf{1 0 0}$ & \\
\hline Total unidentified (mammals) & $\mathbf{6 6 7}$ & & \\
\hline Total & $\mathbf{8 3 8}$ & & \\
\hline
\end{tabular}

Fig. 5.4.1 : Table 1. Distribution of mammal taxa from Kiln (Water Pool) (I. Stojanovitch).

130) Observations personnelles sur la base du matériel étudié.

131) See the corresponding report in Anatolia Antiqua 23 (2015). 


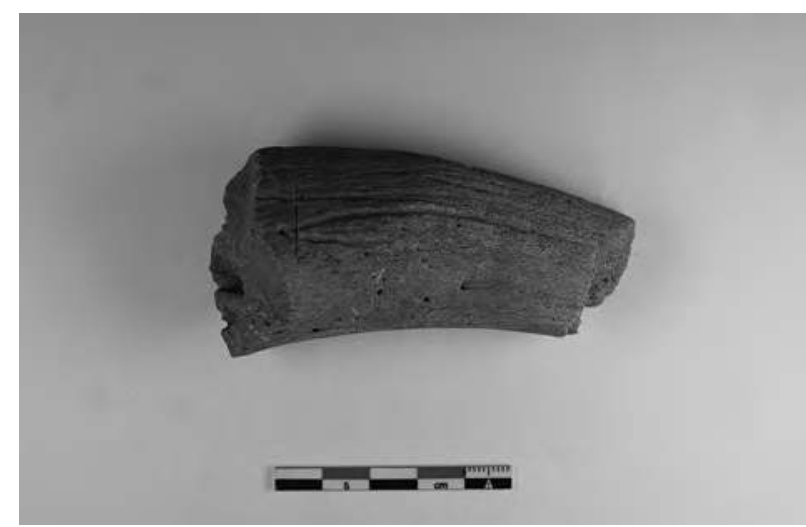

Fig. 5.4.2 : Chop and cut marks on sheep horn core (I. Stojanovitch).

All body parts of ovicaprines are present, including cranial, meat-bearing elements and feet, in similar amount. All different zones of pig skeleton are also identified, dominated by cranial elements. As for the cattle, a complete absence of long bones is noteworthy; isolated teeth are the most numerous; horn core, scapula, pelvis and phalanges are also present.

Almost $70 \%$ of the specimens show traces of discoloration (light brown color). Although it is possible that the changes in color resulted from food preparing (like roasting or boiling), it is more likely that they represent post-depositional modification, related to a specific deposit.

A very few traces of weathering and no gnawing marks indicate rapid burial, i.e. that the bones were not exposed for a long time after they were discarded.

Butchery marks were identified on 35 specimens in form of different cuts and chop marks. For most of them, the species could not be determined, but for the once it could, the most numerous came from different skeletal elements of sheep and goat (mandible, scapula, tibia, metapodial). A number of sheep and goat horn cores were cut in a similar manner, pointing to primary butchery activity (Fig. 5.4.2). Pig specimens with traces of butchery are not numerous, but the diversity of skeletal elements and different cut and chop marks indicate dismembering and food consumption.

Beside mammals, five bird bones were found in the assemblage, which were not identified to a species level on site, but separated for further analyses.

\section{Water Pool}

The faunal material from the excavations of Water Pool (excluding the Kiln) will be presented within two chronological phases -one which consists of mixed material $\left(3^{\text {rd }}-5 / 6^{\text {th }}\right.$ century $\left.A D\right)$ and the other corresponding to the $1^{\text {st }}-2^{\text {nd }}$ century AD Faunal material is in a very good state of preservation, in both phases.

Relative abundance of species is shown on Table 2 (Fig. 5.4.3). Both assemblages are of similar size and it is obvious that they are almost identical in terms of taxonomic frequencies. The only difference is the presence of a hare in the early material.

\begin{tabular}{|c|c|c|c|c|}
\hline & \multicolumn{4}{|c|}{$1^{\text {st }}-2^{\text {nd }}$ c. A.D. $3^{\text {rd }}-5 / 6^{\text {th }}$ c. A.D. } \\
\hline Species/Taxon & NISP & $\%$ NISP & NISP & $\%$ NISP \\
\hline Bos taurus (cattle) & 16 & 7,8 & 10 & 5,3 \\
\hline Sus domesticus(domestic pig) & 21 & 10,3 & 18 & 9,7 \\
\hline Sus sp. (pig indet.) & 1 & 0,5 & & \\
\hline Ovis aries (sheep) & 5 & 2,4 & 10 & 5,3 \\
\hline Capra hircus (goat) & 7 & 3,4 & 13 & 7,0 \\
\hline Ovis/Capra (sheep/goat) & 126 & 61,8 & 119 & 63,6 \\
\hline Equus caballus (horse) & 24 & 11,8 & 17 & 9,1 \\
\hline Lepus capensis (hare) & 4 & 2,0 & & \\
\hline Total identified & 204 & 100 & 187 & 100 \\
\hline $\begin{array}{l}\text { Total unidentified } \\
\text { (mammals) }\end{array}$ & 394 & & 294 & \\
\hline Total & 598 & & 481 & \\
\hline
\end{tabular}

Fig. 5.4.3 : Table 2. Distribution of mammal taxa from Water Pool by phases

(I. Stojanovitch).

In both phases, sheep and goat are the most abundant taxon, followed by horse and pig with similar contribution, while cattle are the least represented domestic species. The only wild species that occurs in the assemblage is hare, with only four specimens attributed to the $1^{\text {st }}-2^{\text {nd }}$ century $A D$

\section{$3^{\text {rd }}-5 / 6^{\text {th }}$ century $A D$}

All body parts of sheep and goat are present, though metapodials stand out as the most numerous skeletal elements, followed by isolated teeth. In the horse assemblage, isolated teeth are the most numerous by NISP, while other skeletal elements appear as more or less individual specimens -long bones, metapodial, phalanx, short bone and pelvis. The remains of pig are not abundant, yet almost all body parts are present, although a complete lack of phalanges and isolated teeth is noteworthy. Remains of cattle are scarce, but presence of cranial, meat bearing elements, so as feet is noted.

Around $10 \%$ of the assemblage $(n=51)$ have traces of butchery. On a large number of sheep and goat remains $(n=28)$ different types of cut and chop 


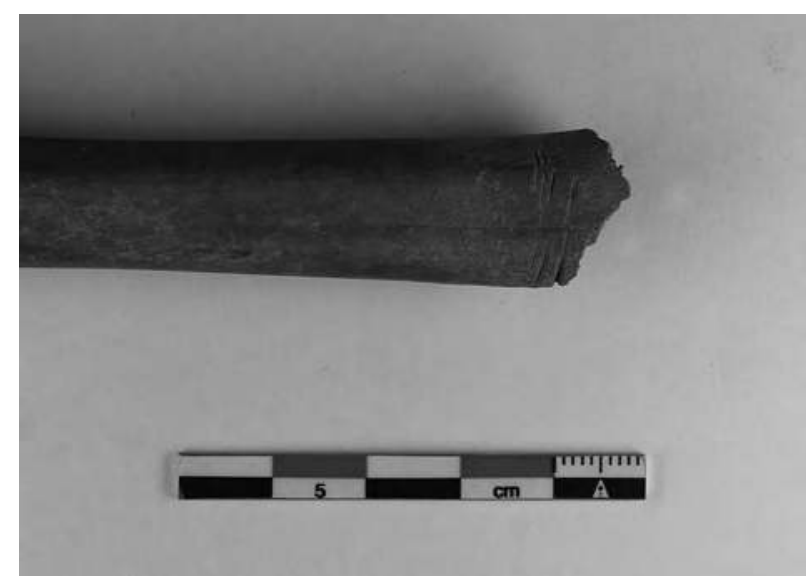

Fig. 5.4.4 : Cut marks on metatarsal of ovicaprine (I. Stojanovitch).

marks were recorded. Interestingly, there is a number of metapodials $(n=16)$ with very similar traces in form of shallow and short transverse, rarely oblique cuts on the diaphysis, related to skinning and/or dismembering practices (Fig. 5.4.4). The same activity was recognized on three horn cores with chopping marks from cleavers. One mandible fragment had clear signs of dismembering. Pig and cattle specimens with cut and chop marks are not that numerous $(n=8)$; they mostly resulted from dismembering and skinning. Butchering marks were observed on 14 specimens that could not be assigned to a species; they mostly consisted of rib and long bone fragments. Some of them can be related to final processing of reducing the carcass into smaller units, including bone breaking and pot sizing.

One metapodial of a horse has butchery traces (Fig. 5.4.5) in form of two discrete, short, transverse cuts on the dyaphisis. This kind of mark on metapodials most likely reflects the process of skinning.

A very few specimens with weathering, burning and gnawing marks were recorded indicating short time of deposition.

Beside mammals, two bird bones were found, so as six fragments of a turtle shield, all from one unit.

For only two sheep and goat specimens approximate age of death could be determined, indicating slaughtering of adult animals (over 3 years of age), which implies that they were used not only for their meat, but also for other products such as milk and fleece.

\section{$1^{\text {st }} 2^{\text {nd }}$ century $A D$}

As in the later phase, all body parts of sheep and goat are present, again with metapodials being more abundant than other elements. Remains of horse con-

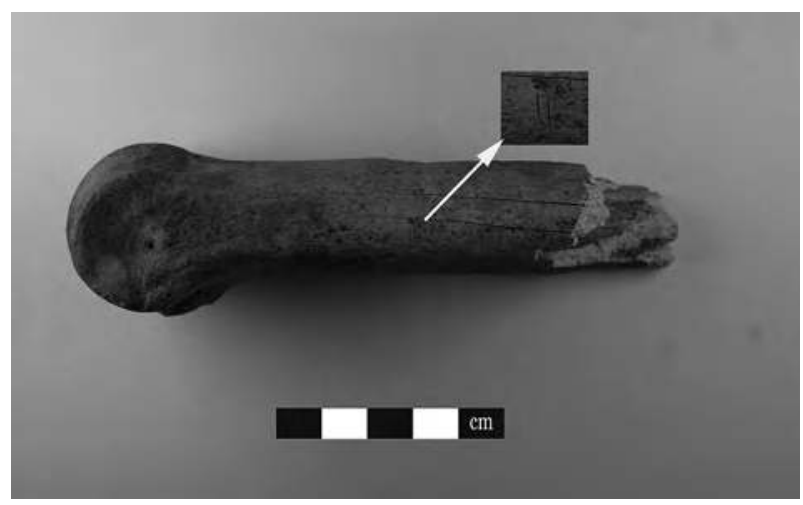

Fig. 5.4.5 : Cut marks on horse metapodial (arrowed) (I. Stojanovitch).

sist of long and short bones and feet in small numbers. The most represented elements are isolated teeth. Although various skeletal elements of cattle are present, they are mostly represented with one to two specimens; almost complete absence of meat-bearing elements was noted. The remains of pig are characterized by domination of metapodials and cranial elements (including teeth); no long bones or phalanges were found in this context. Four specimens of hare, from four different contexts were registered in the assemblage -mandible, femur, tibia and metapodial.

A total of 43 specimens had different marks that resulted from butchering. They are noted on 22 specimens of sheep and goat, mostly on metatarsals and horn cores. Most of them are related to primary butchering activities -skinning and disarticulation. Traces of butchering were recorded on only a few cattle and pig specimens; and on a number of ribs of unidentified species (large and medium mammals).

The assemblage is well preserved, with a small number of bones with gnawing $(n=6)$, weathering $(n=7)$ marks and postdepositional burning $(n=4)$.

As in the later phase, there are no sufficient data to reconstruct age profiles.

Two bird bones are found in the assemblage; cut marks were recorded on one specimen.

\subsubsection{EAST BATH}

General observation is that the material is very fragmented and that only a small part of faunal remains could be determined to a species level. In total, 1.448 specimens were recorded, out of which only 141 could be assigned to a species. Sheep and goat dominate the assemblage by NISP $(68.8 \%)$, followed by cattle $(19.9 \%)$. Few more species are present, but far less in number: domestic pig, wild pig and dog (Table 3: Fig. 5.4.6). 


\begin{tabular}{|l|r|r|}
\hline Species/Taxon & NISP & \% NISP \\
\hline Bos taurus (cattle) & 28 & 19,9 \\
\hline Sus domesticus (domestic pig) & 12 & 8,5 \\
\hline Sus sp. (pig indet.) & 1 & 0,7 \\
\hline Ovis aries (sheep) & 2 & 1,4 \\
\hline Capra hircus (goat) & 7 & 5 \\
\hline Ovis/Capra (sheep/goat) & 88 & 62,4 \\
\hline Canis familiaris (dog) & 2 & 1,4 \\
\hline Sus scrofa (wild pig) & 1 & 0,7 \\
\hline Total identified & $\mathbf{1 4 1}$ & $\mathbf{1 0 0}$ \\
\hline Total unidentified (mammals) & $\mathbf{1 3 0 7}$ & \\
\hline Total & $\mathbf{1 4 4 8}$ & \\
\hline
\end{tabular}

Fig. 5.4.6 : Table 3. Distribution of mammal taxa from East Bath (I. Stojanovitch).

Buchery marks were recorded on 29 specimens ( $2 \%$ of the whole assemblage). A number of bones had specific chop marks, with sharp, smooth edges, possible made with the same/similar tool, probably some kind of a cleaver.

Taphonomic processes did not have a great influence on the preservation of the assemblage -only 17 specimens were slightly weathered; 18 had traces of burning (carbonized and partly carbonized) and just one bone had gnawing marks.

\subsubsection{NORTH $S T O A$}

Small sample and a very few identified specimens from the North Stoa (Table 4: Fig. 5.4.7) do not allow us to discuss the fauna from this context in detail. It seems that it follows the pattern noted on other assemblages - with only domesticated species present among which sheep and goats are the most frequent.

\begin{tabular}{|l|r|r|}
\hline Species/Taxon & \multicolumn{1}{|c|}{ NISP } & \% NISP \\
\hline Bos taurus (cattle) & 1 & 4,0 \\
\hline Sus domesticus (domestic pig) & 1 & 4,0 \\
\hline Ovis aries (sheep) & 1 & 4,0 \\
\hline Ovis/Capra (sheep/goat) & 22 & 88,0 \\
\hline Total identified & $\mathbf{2 5}$ & $\mathbf{1 0 0}$ \\
\hline Total unidentified (mammals) & $\mathbf{7 5}$ & \\
\hline Total & $\mathbf{1 0 0}$ & \\
\hline
\end{tabular}

Fig. 5.4.7 : Table 4. Distribution of mammal taxa from North Stoa (I. Stojanovitch).

\section{CONCLUSION}

Even though all assemblages are small in zooarchaeological terms (NISP up to 200) we can make some general conclusions and discuss specific phenomenon.

For the most part, faunal remains represent butchery and consumption waste, based on context data, fragmentation, skeletal elements and butchery marks. They can be considered as secondary material, which is not directly related to the function of the excavated buildings.

All contexts represent sheep and goat dominant assemblages. It seems that a meat diet was based on mutton and goat meat, while pork and beef were much less consumed. There is an apparent lack of wild animals, which might be the result of limited space investigated or different disposal practices.

Distribution of skeletal elements and characteristics of butchery marks suggest that all parts of the ovicaprine carcass were utilized, and that these animals were most likely killed and butchered on site. Butchery marks indicate the use of both cleavers and knives for skinning, disarticulation, meat removal and bone breaking, suggesting that butchery technology was efficient.

Although cut marks (created by skinning) were noted on only one specimen of a horse, they confirm the use of these animals other than the means of transportation. It is still an open question if their meat was consumed.

Future zooarchaeological research in Labraunda will continue to focus on contextual analyses, investigation of food and discard practices, but also on the economy and subsistence strategies throughout its long period of occupation and use.

\subsection{MATERIEL METALLIQUE \\ (par E. Goussard)}

L'étude du mobilier métallique réalisé lors de la campagne 2017 à Labraunda comportait deux objectifs : il s'agissait tout d'abord de créer un inventaire répertoriant toutes les pièces métalliques du site mise au jour depuis le début des fouilles en 1948 et conservées sur le site. Ce recensement est une première étape dans la mise en place d'un socle documentaire homogénéisant les données des fouilles anciennes et récentes, nécessaire à l'analyse globale du mobilier métallique de ce site.

Cette étude avait également pour but de réaliser un état sanitaire de la collection, afin de pallier aux éventuels problèmes de conservation de ces pièces métalliques. 


\subsubsection{METHODE MISE EN PLACE}

Pour atteindre ces objectifs, un tableau d'inventaire a été mis en place. Il se divise en trois sections :

1) Les informations relatives à l'objet luimême : cette section contient le numéro d'ordre attribué à l'objet, le matériau, le poids, le nombre de fragments dont il est composé, la présence ou non de restes organiques minéralisés, l'état de conservation, une brève description et une proposition d'identification. Des lots d'objets ont été créés pour des objets de même nature, provenant du même contexte (comme un lot de clous par exemple). Dans ce cas, le numéro d'ordre a été attribué au lot.

2) La localisation de l'objet au moment de sa découverte : sont reportés les différents marquages (étiquette, marquage sur l'objet) et l'année de fouille.

3) Les informations indiquant son lieu de conservation : sont mentionnés le numéro de boîte dans lequel l'objet a été placé, sa localisation avant reconditionnement du métal (facilitant la reconstitution des assemblages avec les autres matériaux) et l'indication de son passage ou non au musée d'Izmir.

L'indication de l'état de conservation des objets se décline en deux mentions : "stable" et "corrosion active". Lorsque l'objet nécessite une restauration urgente, un label "prioritaire" a été ajouté.

Enfin, pour limiter les risques de casse et d'altération mécanique, les objets inventoriés ont été reconditionnés (Fig. 5.5.1). Les objets fragiles ont d'abord été protégés par du papier bulle. Le mobilier a ensuite été placé dans des sachets de reconditionnement type "minigrip". Ces contenants présentent l'avantage, au contraire des sacs en papier ou cartons, de ne pas être acides (et donc agressifs pour le métal), ni putrescibles. Leur transparence permet également une veille sanitaire du mobilier plus aisée. Tous les anciens marquages ont été reportés.

Dernière étape, ces sachets ont été placés sans être compressés, pour éviter les altérations dues à une trop forte pression, dans des boîtes plastiques à couvercle. Ces dernières permettent de limiter les risques d'intrusion et de mélange.

\subsubsection{RESULTATS}

563 objets, correspondant à 1830 fragments ou 33,893 kilogrammes de métal, ont été inventoriés cette année. Ils proviennent des fouilles des années
$1945^{132}, 1948-1960,1988-1991,2010$ et 2012-2016. Il est important de noter que toutes les identifications proposées ne sont que préliminaires. Elle seront à confirmer après l'étude typologique du mobilier, qui interviendra à partir de la campagne 2018.

\section{Premières identifications}

324 (soit plus de $57 \%$ du corpus) de ces objets sont en fer (Fig. 5.5.2). Il s'agit, selon la première identification, de 39 armes (pointes de lances, de flèches et lames), de 8 couteaux, de 2 rasoirs, de 4 éléments de parure (fibules et bagues), de 19 scories, de 218 éléments de quincaillerie (éléments de penture, clous, agrafes, crampons, pitons, appliques) et 34 éléments encore indéterminés.

200 (ou près de $36 \%$ du corpus) sont en alliage cuivreux. Trois d'entre eux ont été dorés. 8 de ces éléments en alliage cuivreux sont des éléments de statue ou de statuettes. Ils s'agit aussi de 12 vases (notamment des kylix, écuelles et skyphos), 3 clochettes en bronze, 11 armes (pointes de flèches, appliques de bouclier), 39 éléments de parures (bagues, boucles d'oreille, fibules, bracelets, broches, pendeloques), 6 instruments de toilette, 12 monnaies, 19 objets en cours de fabrication, 52 éléments de quincaillerie (dont 15 décors de clous de porte) et 38 objets dont il faudra préciser l'identification.

Enfin, 39 objets en plomb ont été identifiés. Il s'agit d'un fragment de vase, de 7 scellements (dont deux enserrant encore des fragment de pithoï), 16 rebuts de fabrication (notamment des coulées), 3 plaques percées, 5 éléments de quincaillerie (clous, fiches et crampons) et 7 éléments indéterminés.

\section{Restes organiques minéralisés}

Sur ces 563 objets, 25 éléments (ou un peu plus de $4 \%$ du corpus) comportaient des restes organiques minéralisés dans leurs produits de corrosion. Parmi eux, 22 sont en fer et 3 en alliage cuivreux.

Il s'agit de restes de bois, parfois en position fonctionnelle, comme sur le manche d'un couteau $\left(\mathrm{n}^{\circ} 265\right)$ ou à l'intérieur de la douille de la pointe de lance ( $\mathrm{n}^{\circ}$ 554). Des traces de bois ont également été repérées en position non fonctionnelle, comme sur la flamme de la pointe de flèche ou de javeline $\left(\mathrm{n}^{\circ}\right.$ 340), indiquant son abandon (et donc sa corrosion) contre un élément en bois. Enfin, des traces de brindilles ont été repérées, comme sur une des faces de la pointe de lance $\left(\mathrm{n}^{\circ} 346\right)$. 

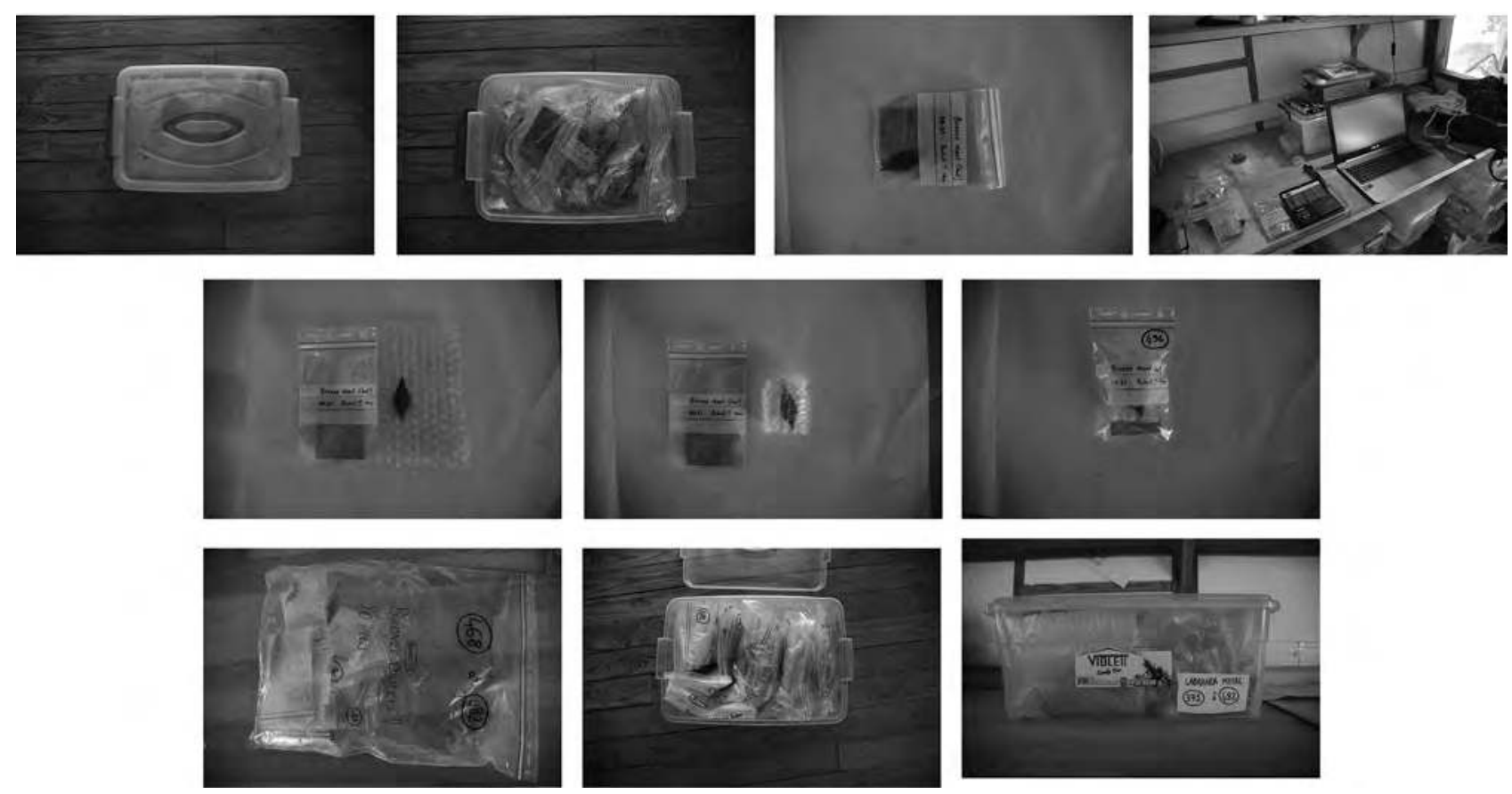

Fig. 5.5.1 : Différentes étapes de l'étude du mobilier métallique de Labraunda : de l'enregistrement au reconditionnement (E. Goussard).

Cette première observation des restes organiques minéralisés, effectuée à l'œil nu, ne permet que de repérer les fibres végétales. Elle sera complétée lors de la prochaine campagne par une observation sous loupe binoculaire, donnant la possibilité d'identifier les textiles, les fourrures et les cuirs (selon la méthode mise en place par C. Proust) ${ }^{133}$. Cette étude des restes organiques minéralisés permettra une meilleure restitution des objets et de leur contexte d'abandon.

\section{Etat sanitaire de la collection}

Les objets inventoriés présentent majoritairement des signes de corrosion active et risquent donc de s'altérer au cours des années à venir (Fig. 5.5.3). Seuls 135 (soit environ $24 \%$ du corpus) sont stables. Parmi 428 objets dont la corrosion est active, 131 présentent un stade d'altération avancé (label "prioritaire"). Cela représente environ $23 \%$ des objets présents dans l'inventaire. Pour les conserver, ces objets devront bénéficier d'une stabilisation (au chlorure d'argent pour les alliage cuivreux et en bains de sulfite alcalin en solution pour les ferreux).

133) Proust 2009.

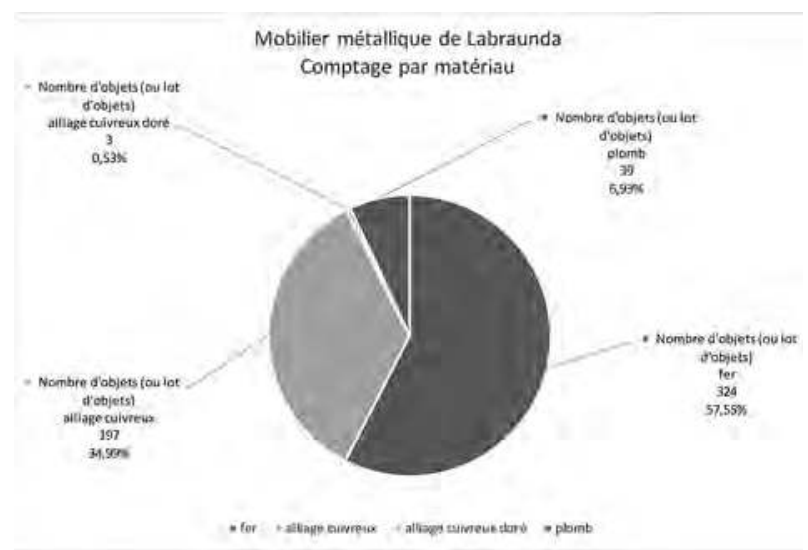

Fig. 5.5.2 : Comptage par matériau du mobilier métallique de Labraunda (E. Goussard).

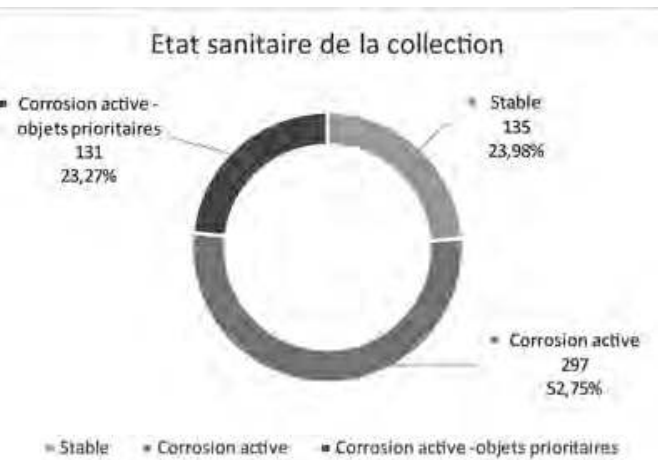

Fig. 5.5.3 : Etat sanitaire du mobilier métallique de Labraunda (E. Goussard). 


\section{Perspectives}

Le travail effectué en 2017 ne constitue que la première étape d'une étude plus large et plus complète du mobilier métallique de Labraunda.

En effet, l'inventaire du mobilier métallique doit être complété. Il reste encore à intégrer le mobilier découvert en 1992, 1993, 2011 et 2017 (correspondant à 248 objets ou lots d'objets). Ces objets seront également reconditionnés, afin de limiter les altérations mécaniques.

Une prise de dimensions et une documentation graphique systématique, par photographie et/ou par dessin, de chaque objet devra ensuite être associé à l'inventaire. Cette documentation permettra ainsi d'effectuer une analyse typologique et donc une datation de ce mobilier. Cette étude pourra être complétée par une analyse des restes organiques minéralisés, mais aussi par des analyses métallographiques sur les scories et les objets en cours de fabrication, qui permettront de mieux comprendre la production métallique à Labraunda.

\section{6. $A N D R \hat{O} N$ MOSAÏQUE (par A.-M. Guimier-Sorbets)}

A l'occasion de la campagne 2017 des fouilles, et à la demande d'Olivier Henry, j' ai étudié les fragments de mosaïques de galets qui avaient été découverts en 2014/2015 à l'intérieur de l'Andrôn A, lors des travaux de fouille (Fig. 5.6.1). Ces fragments avaient été photographiés par O. Henry et il avait pu faire réaliser quelques analyses.

Par l'étude sur place de ces fragments de petite taille qui ne constituent qu'une partie réduite de la surface originelle de la pièce, nous avons pu :

- confirmer que ces documents datent bien de la période de construction du monument, vers le milieu du $4^{\mathrm{e}}$ s. av. J.-C. ;

- trouver des parallèles grecs de cette technique de mosaïques de galets insérés dans un mortier qui avait été ensuite recouvert d'une couche colorée en rouge rosé ;

- formuler des hypothèses bien étayées sur la nature de la composition du pavement (tapis principal et tapis de seuil) et de son décor figuré, géométrique et végétal. Parmi les éléments du décor, on peut vraisemblablement reconnaître la présence d'au moins un fauve et de palmettes.

Ces petits fragments présentent un grand intérêt puisqu'ils permettent de restituer la nature et une partie au moins du décor du pavement de l'Andrôn A, bâtiment de prestige dont l'importance n'est plus

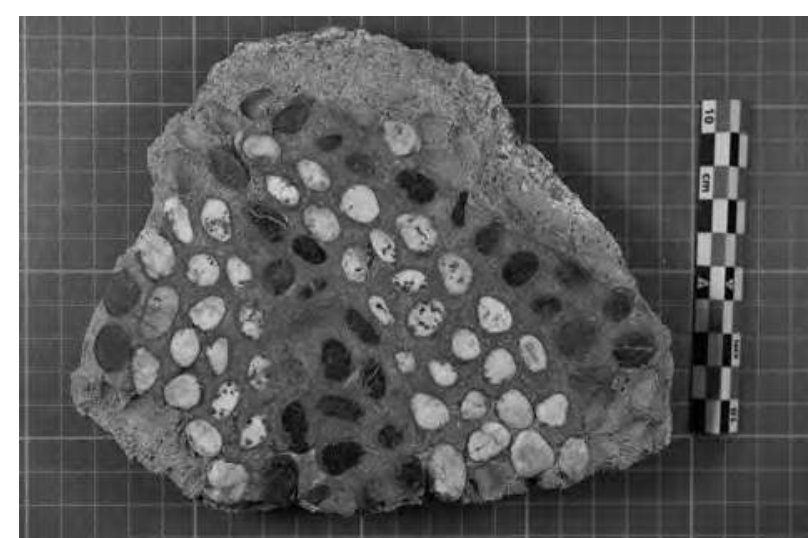

Fig. 5.6.1 : Exemple de fragment de mosaïque de galets mis au jour lors des fouilles de l'Andrôn A en 2015 (O. Henry).

à démontrer. Ils permettent aussi de montrer que, même pour le pavement, le roi carien qui en était le commanditaire avait eu recours à une équipe d'artisans grecs.

Les résultats plus détaillés de ce travail seront publiés, avec les documents de comparaison, dans un article que je co-signerai avec Olivier Henry.

\subsection{LA STOA EST}

(par N. Carless-Unwin et B. Vergnaud)

Au cours de la campagne 2017, un nouveau projet a été initié dans la partie est du site de Labraunda. Celui-ci a pour but de procéder à une étude complète de la Stoa est (East Stoa) et de la terrasse sur laquelle elle fut installée. Les fouilles d'ampleur limitée, réalisées par les archéologues suédois à la fin des années 1980 et au début de la décennie suivante, ont permis de mettre au jour le plan de la stoa et de dater sa construction vers le milieu du $4^{\mathrm{e}} \mathrm{s}$. av. J.-C. Aucune fouille n'a été réalisée sur la très grande esplanade s'étendant à l'Ouest de la stoa mais des prospections géophysiques récentes ont permis de révéler l'existence d'une construction de plan rectangulaire dont la fonction reste inconnue mais qui pourrait avoir fonctionné en relation avec la stoa. Le nouveau projet vise à procéder à une étude stratigraphique et architecturale complète de ces deux ensembles afin de préciser d'une part la datation et les différentes phases d'occupation de la stoa et, d'autre part, la fonction ainsi que la datation de la construction située sur la terrasse. Ces nouvelles recherches s'inscrivent plus généralement dans la réflexion sur le développement du site qui a connu une grande phase de monumentalisation sous l'impulsion des Hécatomnides. Il s'agit donc d'évaluer la place de cette partie encore méconnue du site 
de Labraunda dans ce processus de d'aménagement ininterrompu qu'a connu le site entre le deuxième quart du $4^{\mathrm{e}} \mathrm{s}$. et l'époque romaine impériale.

\subsubsection{LA STOA ET LA TERRASSE : DESCRIPTION ET RECHERCHES PRECEDENTES}

La stoa se situe à l'extrémité orientale du site, à environ $3 \mathrm{~m}$ du mur de péribole (Fig. 5.7.1). Elle s'étend du Nord au Sud sur une distance d'environ $45 \mathrm{~m}$ et sa largeur atteint $13 \mathrm{~m}$ (Fig. 5.7.2). Le bâtiment est principalement constitué de gneiss. Les deux piliers d'ante et les 17 colonnes de marbre supportaient un entablement dorique consituté du même matériau, bien que la présence de pièces de bois soit envisagée. Les 6 pièces situées derrière la colonnade ont été identifiées comme des salles de banquet. En effet, la porte est légèrement désaxée pour permettre la mise en place des klinè dont le nombre est évalué à 11 par pièce. Les fouilles précédentes (1991) ont mis au jour la totalité du stylobate, les colonnes et un certain nombre de fragments architecturaux (Fig. 5.7.3). La présence d'un four à chaux tardif découvert devant la stoa peut expliquer en partie la rareté des marbres dans le secteur. Les fouilles ont permis d'atteindre les fondations du stylobate et la céramique découverte en association avec le mur ont permis de fixer une datation vers le milieu du $\mathrm{IV}^{\mathrm{e}} \mathrm{s}$. et d'attribuer la construction de la stoa aux Hékatomnides. Les fouilles anciennes, qui se sont concentrées sur la partie nord de la stoa, ont permis de mettre au jour du matériel qui s'échelonne entre le $\mathrm{IV}^{\mathrm{e}} \mathrm{s}$. et l'époque romaine, un laps de temps qui pourrait correspondre à la durée d'occupation du bâtiment. Ce dernier semble avoir été abandoné avant l'époque romaine tardive puisqu'une maison datée de cette période fut construite devant le stylobate, dans l'angle formé par le mur de terrasse nord et la stoa. Cette habitation fut notamment construite avec les remplois de la stoa. Enfin, il est à noter qu'aucune fouille n'a été réalisée à l'intérieur des pièces de la stoa. La datation de leur occupation et leur organisation interne restent donc à déterminer.

La construction située sur la terrasse en avant de la colonnade, révélée par des prospections géophysiques demeure énigmatique. Ces derniers ont révélé l'existence de plusieurs murs situés à quelques centimètres de la surface, dont certains forment une grande construction rectangulaire dont notamment une plate-forme carrée. L'hypothèse a été avancée que ces aménagements pourraient correspondre à un temple et à un autel. Il ne s'agit cependant que d'une

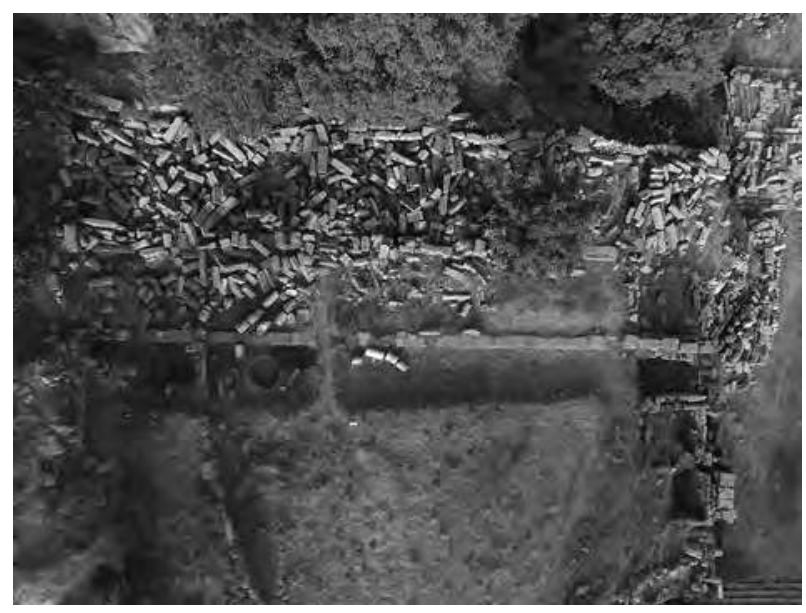

Fig. 5.7.1 : Vue aérienne de la Stoa Est (O. Henry).

hypothèse de travail que nous chercherons à vérifier dans le cadre des campagnes à venir, de même que nous viserons à établir sa relation avec la stoa.

\subsubsection{LA CAMPAGNE 2017}

La campagne 2017 s'est déroulée sur 2 semaines au cours desquelles nous avons procédé à un nettoyage complet du secteur nord, celui des fouilles anciennes (Fig. 5.7.4), de la pièce $\mathrm{n}^{\circ} 2$ ainsi que du mur de péribole et de l'espace qui sépare ce dernier du mur arrière de la stoa. Ces travaux ont été menés afin de préciser le plan du bâtiment et en préparation de fouilles que l'on espère pouvoir mener pendant la campagne 2018 .

Les travaux ont débuté avec le nettoyage du mur de péribole autrefois complètement masqué par la végétation (Fig. 5.7.5), et l'espace entre celui-ci le mur de la stoa. Le mur de péribole est un mur pseudo-isodome construit en carreaux et boutisses qui ne présente pas de particularités qui permettraient de proposer une datation précise. Son tracé est parallèle au mur arrière de la stoa dont il est séparé d'environ $3 \mathrm{~m}$. Le nettoyage de cet espace et du sommet du mur arrière de la stoa nous a permis de mettre au jour un lot de céramiques appartenant à des horizons chronologiques très différents, de l'époque archaïque à l'époque romaine, ainsi que des fragments de tuiles. Il est pour l'instant trop tôt pour savoir comment cet espace était utilisé mais il est possible d'envisager qu'il ait pu être lié au fonctionnement de la stoa, peut-être comme espace de stockage et de préparation pour les banquets. Cette idée peut être supportée par la découverte de plusieurs fragments de pithoi ainsi que par la présence d'un escalier de service qui semblait mener à l'arrière de la stoa depuis les propylées est (Fig. 5.7.6). Les preuves sont encore maigres 


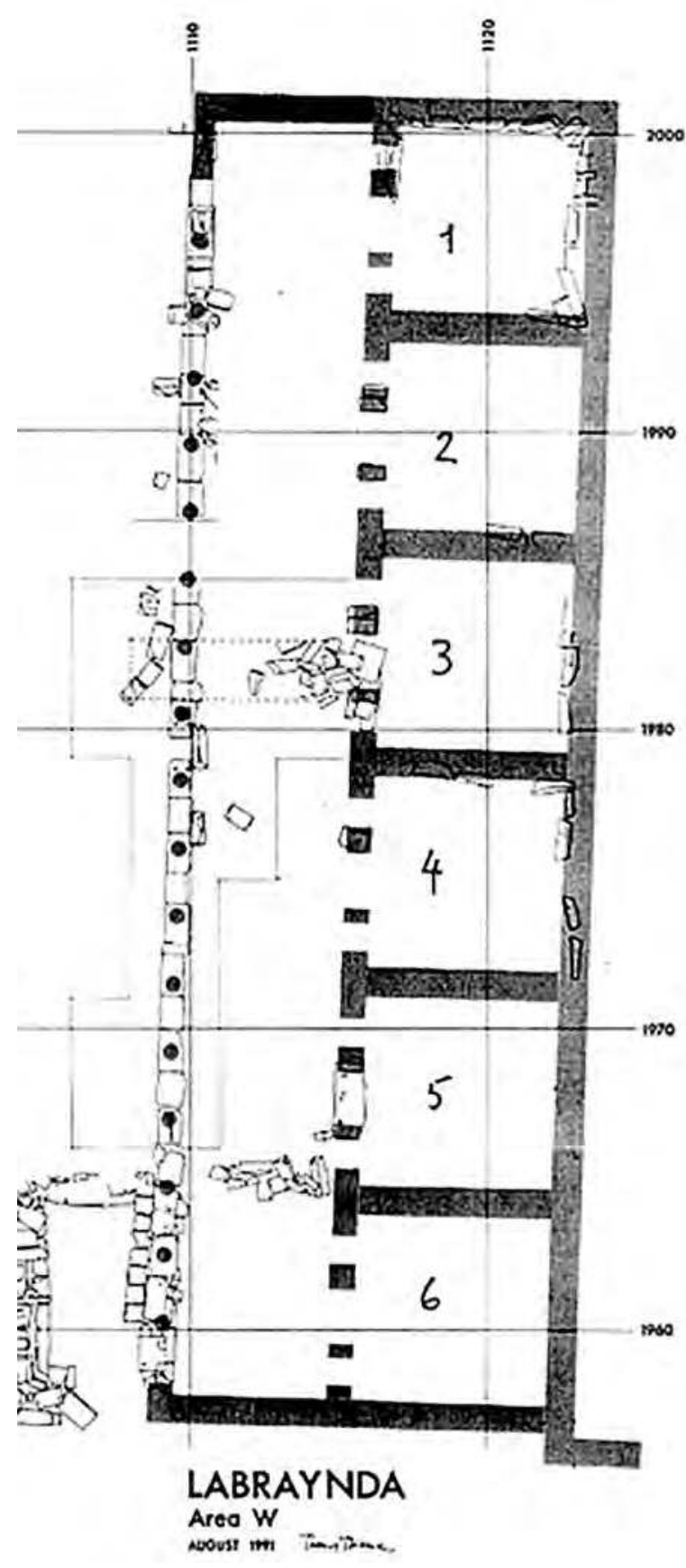

Fig. 5.7.2 : Plan de la Stoa Est

(Mission archéologique de Labraunda 1991).

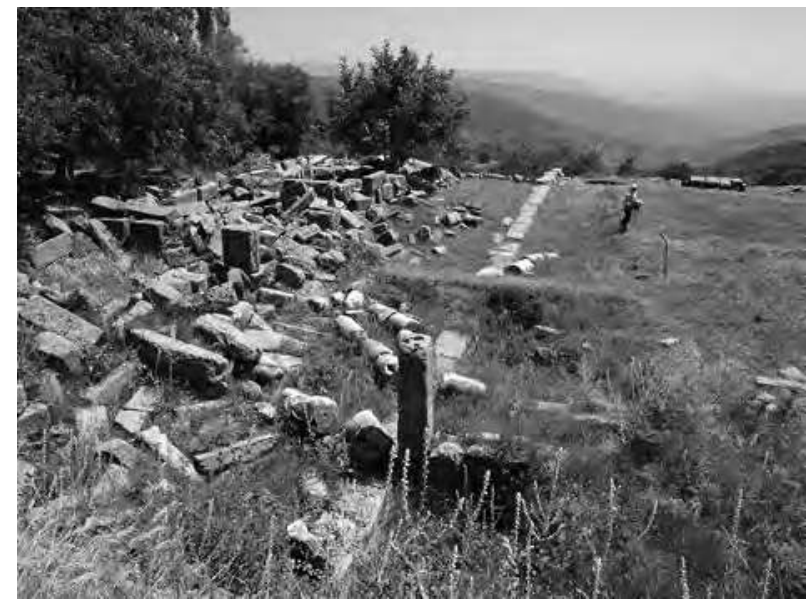

Fig. 5.7.3 : La Stoa Est avant le début des travaux (B. Vergnaud et N. Careless-Unwinn).

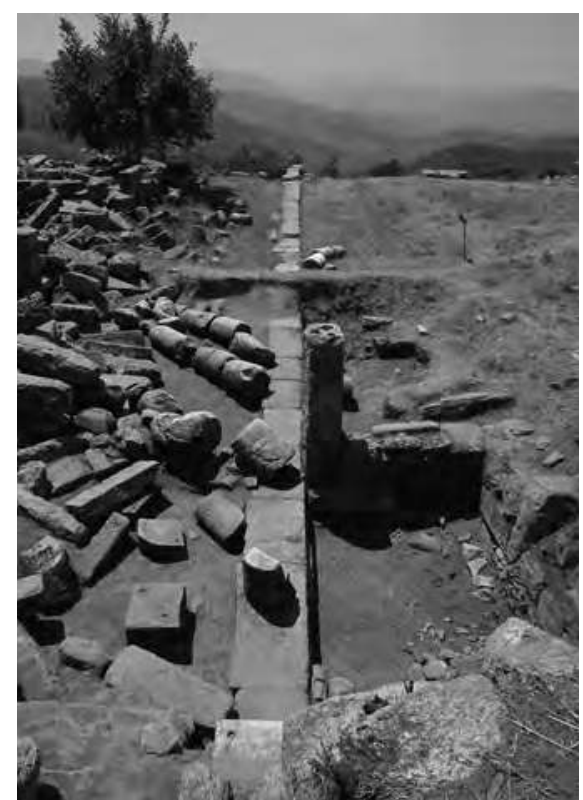

Fig. 5.7.4 : Le stylobate et le secteur des fouilles anciennes après nettoyage 2017

(B. Vergnaud et N. Careless-Unwinn).

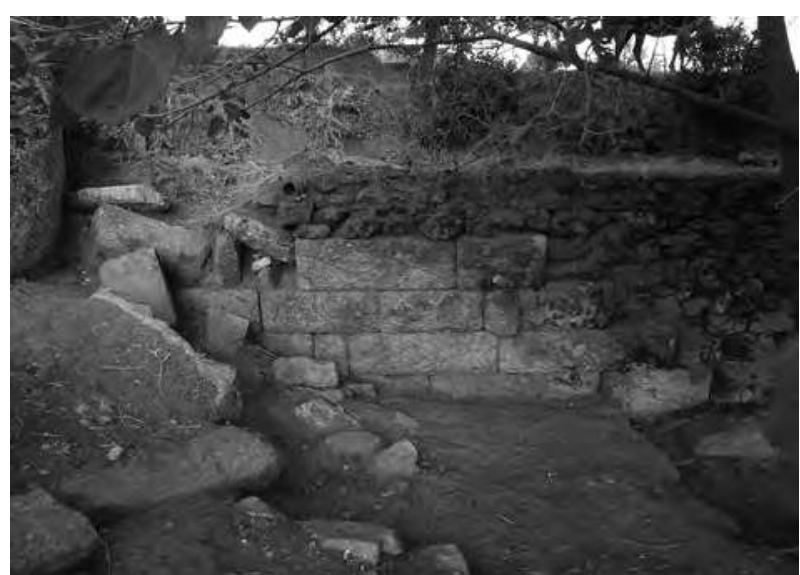

Fig. 5.7.5 : Le mur de péribole après nettoyage $(B$. Vergnaud et N. Careless-Unwinn). 
et seules des fouilles nous permettraient de confirmer cette hypothèse de travail. Une telle interprétation est cependant privilégiée pour ce qui concerne l'espace situé à l'arrière de l'Andrôn A et du bâtiment des $O i$ koi à l'Ouest du site.

Les opérations de nettoyage se sont également portées sur l'intérieur de la pièce $\mathrm{n}^{\circ} 2$ que nous avons débarassée de la végétation et de la terre végétale (Fig. 5.7.7). Ces travaux nous ont permis de mettre au jour de nombreux fragments de céramiques, en particulier des fonds de coupes à boire qui paraissent liées au fonctionnement de la pièce comme salle de banquet. Les tuiles mises au jour appartiennent sans doute à la toiture du bâtiment original. Nos travaux ont également permis de révéler l'existence d'un aménagement qui pourrait être interprété comme un foyer situé à environ $1,5 \mathrm{~m}$ du seuil de la pièce. Nous ignorons si ce foyer est un ajout tardif ou s'il appartient à la phase initiale d'utilisation de la salle. Le dégagement des très nombreux blocs qui obstruent la pièce devrait nous permettre de procéder à des fouilles dans cet espace et en connaître plus quant à son organisation et à sa chronologie.

Les pièces de la stoa semblent avoir toutes été bâties de la même façon : une porte désaxée ouvre sur un espace éclairé par deux fenêtres placées de part et d'autre de la porte. Une de ces fenêtres est conservée dans la pièce $\mathrm{n}^{\circ} 1$ (Fig. 5.7.8). L'une de ces pièces, la pièce $\mathrm{n}^{\circ} 6$, a subi une lourde destruction. Elle était soutenue par les murs du "so-called palace", sur lequel le mur de stylobate repose encore (Fig. 5.7.9). La destruction de la partie est de ce bâtiment monumental a donc entraîné la destruction partielle de l'extrémité sud de la stoa. Malgré ces difficultés, nous tenterons d'établir la relation entre la stoa et le "so-called palace" dans le cadre de notre enquête architecturale.

Parmi les découvertes que nous avons effectuées, il convient de mentionner la mise au jour de trois fragments architecturaux. Le premier, en marbre, a été découvert sur le mur arrière de la stoa et il s'agit à n'en pas douter d'un denticule (Fig. 5.7.10). Le second, également en marbre, présente une entaille de forme carrée et a été découvert à l'intérieur de la pièce $\mathrm{n}^{\circ} 2$. Nous n'avons pas pu l'identifier avec sécurité mais il est possible qu'il ait appartenu comme le précédent à l'entablement du bâtiment. Enfin, une plaque carrée faite de gneiss et présentant des encoches prévues pour sa fixation a été découverte en chute sur le mur de stylobate (Fig. 5.7.11). Il s'agirait vraisemblablement d'un élément de plaquage pour l'une des fenêtres des pièces de la stoa. De nombreuses questions demeurent quant à l'architecture de la stoa, en particulier en ce qui concerne l'entablement de cette dernière. Si un chapiteau d'ante a été retrouvé au cours des fouilles anciennes, aucun des chapiteaux de la colonnade n'a encore été mis au jour. Il est possible que ces derniers aient été utilisés dans le four à chaud mais de plus amples fouilles pourraient permettre probablement d'en trouver des fragments et nous autoriser à restituer avec plus de précision l'apparence de la stoa.

\section{CONCLUSION}

La campagne 2017 avait principalement pour but d'évaluer avec précision l'ampleur du travail à accomplir dans le futur en initiant la reprise d'un dossier important, celui d'un bâtiment unique à Labraunda et peut-être même dans l'Asie Mineure pré-hellénistique. Ces recherches portent sur la stoa, son environnement immédiat -la terrasse et la construction révélée par la géophysique- et sa situation dans le site. Les fouilles que nous souhaitons entreprendre dans le futur auront pour but de parvenir à une meilleure connaissance de l'architecture de la stoa mais aussi de son histoire. Il s'agira de déterminer les différentes phases d'occupation tout en tentant d'identifier des changements dans l'occupation du bâtiment. Une partie des réponses pourra être apportée avec la publication complète du matériel des fouilles anciennes qui reste encore inédit. Nous chercherons également à connaître la relation de la stoa avec le bâtiment enfoui sous la terrasse adjacente et avec le "so-called palace", l'un des bâtiments les plus emblématiques de Labraunda et sur lequel la stoa repose en partie. Ces deux constructions semblent contemporaines et elles peuvent avoir fonctionné ensemble. Ce nouveau projet vise à procéder à une approche globale en ce qu'il s'inscrit dans une réflexion plus large sur l'aménagement du site par les Hékatomnides. Le Labraunda East Stoa Project est un apport important à ce programme de recherche en ouvrant la partie orientale du site, encore largement inexplorée, et devrait permettre de mieux comprendre le projet des Hékatomnides sur le site, à la fois comme dynastes cariens et satrapes perses. Ces nouvelles recherches s'inscrivent dans la lignée de la réflexion initiée par L. Karlsson et poursuivie par O. Henry selon laquelle le site de Labraunda pourrait avoir été aménagé non seulement comme un sanctuaire mais aussi comme un espace de type palatial selon le modèle achéménide ${ }^{134}$. La reprise de l'enquête de la stoa et de sa terrasse 


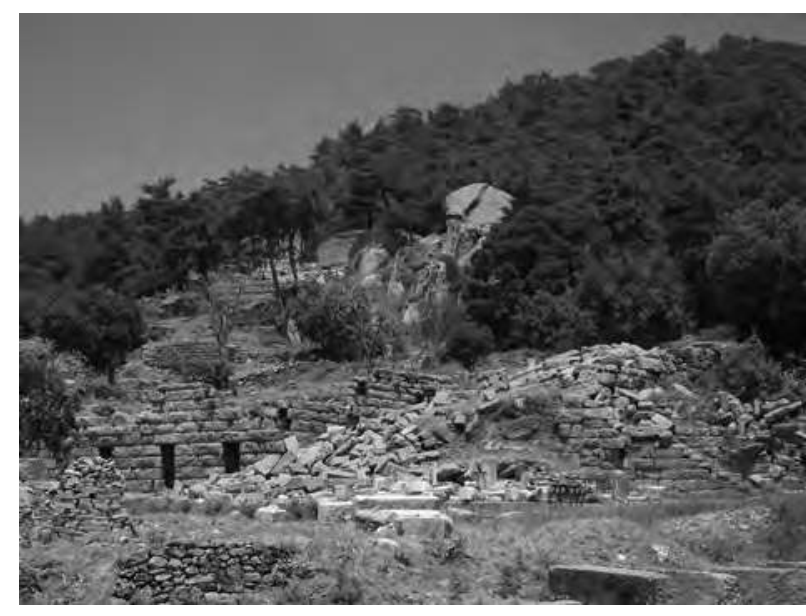

Fig. 5.7.6 : Escalier menant à l'arrière de la stoa (B. Vergnaud et N. Careless-Unwinn).

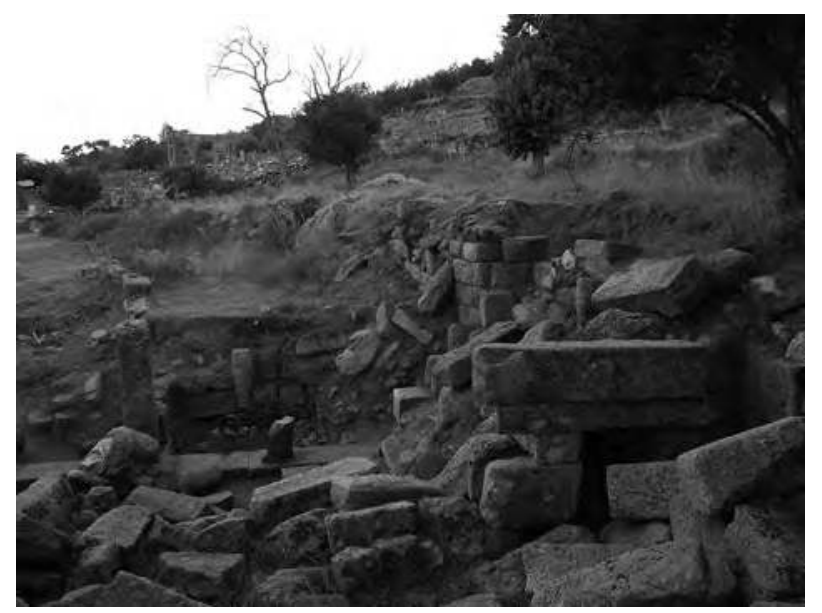

Fig. 5.7.8 : Fenêtre de la pièce $n^{0} 1$ (B. Vergnaud et N. Careless-Unwinn).

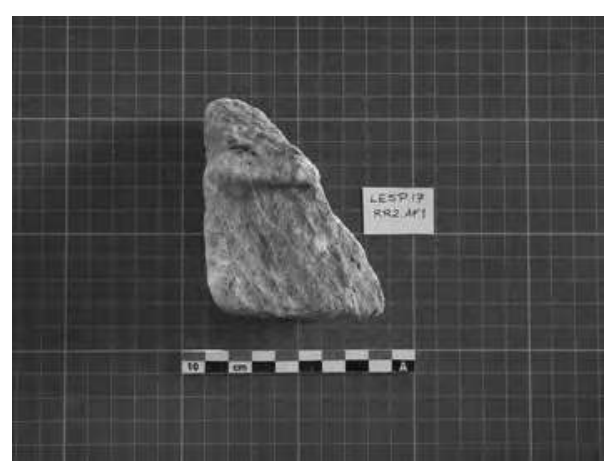

Fig. 5.7.10 : Denticule

(B. Vergnaud et N. Careless-Unwinn).

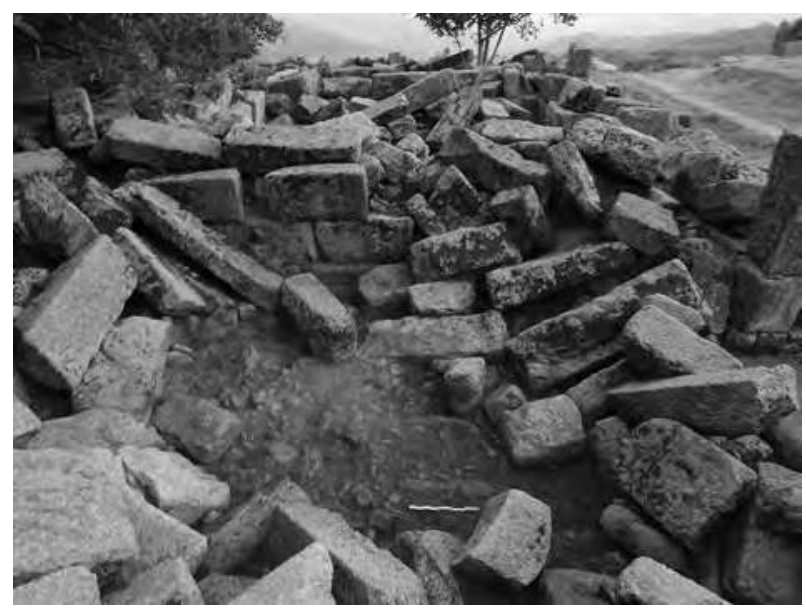

Fig. 5.7.7 : La pièce $n^{0} 2$ après nettoyage (B. Vergnaud et N. Careless-Unwinn).

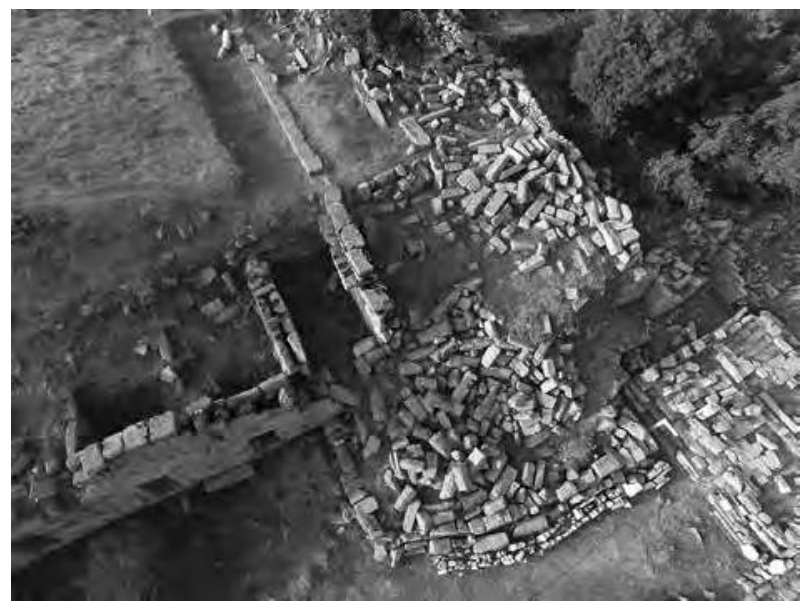

Fig. 5.7.9 : Pièce $n^{0}$ 6, stylobate et so-called palace (O. Henry).

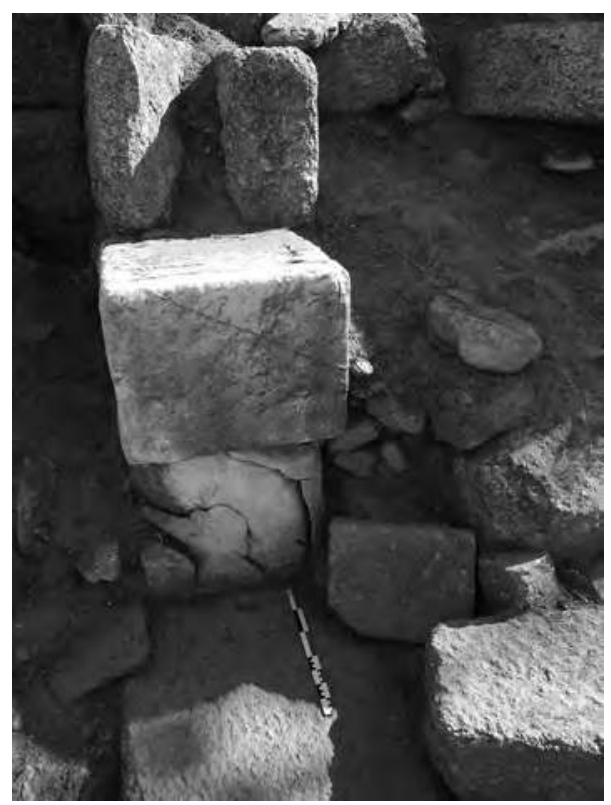

Fig. 5.7.11 : Element de plaquage découvert au pied du pilier d'ante (B. Vergnaud et N. Careless-Unwinn). 


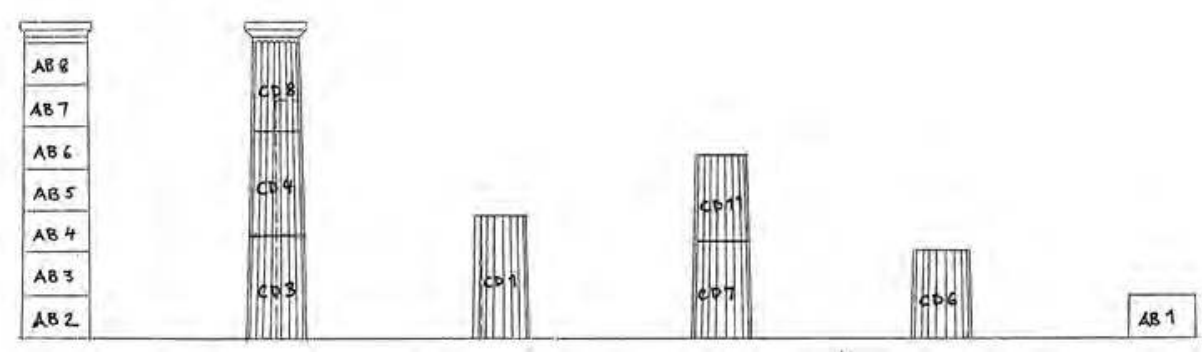

Fig. 5.8.1 : Etat actuel de la façade des Oikoi (T. Thieme).

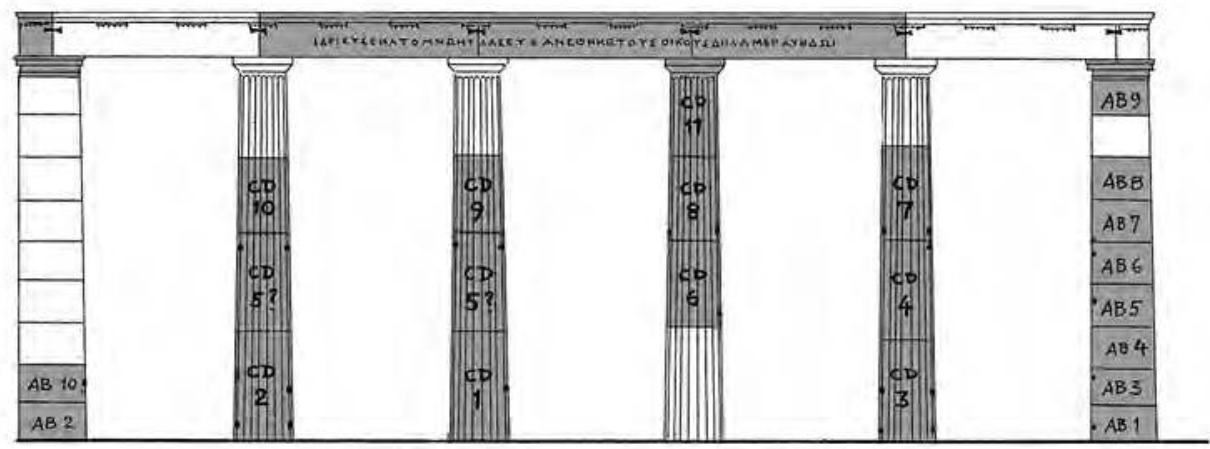

Fig. 5.8.2 : Projet d'anastylose de la façade des Oikoi (en grisé les blocs disponibles sur le site) (T. Thieme).

devrait donc permettre de clarifier la nature du patronage dynastique à Labraunda et de mettre en lumière les différentes fonctions du site au $4^{\mathrm{e}} \mathrm{s}$.

\subsection{OIKOI (par O. Henry)}

Notre projet de restauration/mise en valeur des Oikoi - mis en route en 2016 avec le nettoyage des murs du bâtiment, le renforcement et la protection des piliers d'angle de la pièce sud, le traitement de la dédicace architravale et son placement sur une plate-forme dédiée, ainsi que la protection des sols - s'est poursuivi en 2017. L'objectif de ce projet est de procéder in fine, à l'anastylose de la façade du bâtiment, de la base des colonnes aux architraves (Fig. 5.8.1-5.8.2). L'analyse du bâti a été faite par P. Hellström il y a quelques années déjà, manquait encore d'effectuer une campagne de relevés visant à documenter l'ensemble des membres architecturaux (hors architrave, déjà relevée) de ladite façade. C'est chose faite en 2017 avec la production de relevés au $1 / 10^{\text {e }}$ de 22 blocs d'architectures comprenant 12 tambours de colonne et 10 blocs d'ante.

\subsection{WALL DECORATION MATERIALS AND TECHNIQUES (par A. Freccero)}

Fragments of decorated wall plaster have, so far, been found in two buildings at Labraunda: in the Andrôn A and in the East Church ${ }^{135}$. The find contexts are dissimilar and the fragments suspected to date from different periods. Ocular examination of the plasters in situ permitted the identification of one decoration period at Andrôn A and two phases in the East Church. Each decoration period is represented by a specific type of plastering and decoration.

The present investigation, which began in 2010 with the cleaning and examination of a large collection of East Church fragments, and continued in 2015-17 with the examination of five Andrôn A fragments, concerns in total nine plaster samples from both contexts. The objective was not only to understand the development of decoration technology at Labraunda, but also to investigate a possible link between the Labraunda decorations and plasters which I had already investigated in four other contexts ${ }^{136}$. Hopefully, it would be possible to add

135) The present study was carried out in 2015-17. In the same period I received funding for laboratory analyses of nine samples.

136) The Villa of Livia at Prima Porta in Rome, 32 buildings at Pompeii, the Roman villa at Ossaia, Cortona and the Roman villa at Agios Donatos in Greece. I had also studied decorated plaster fragments found underneath the church San Lorenzo in Lucina in Rome. 


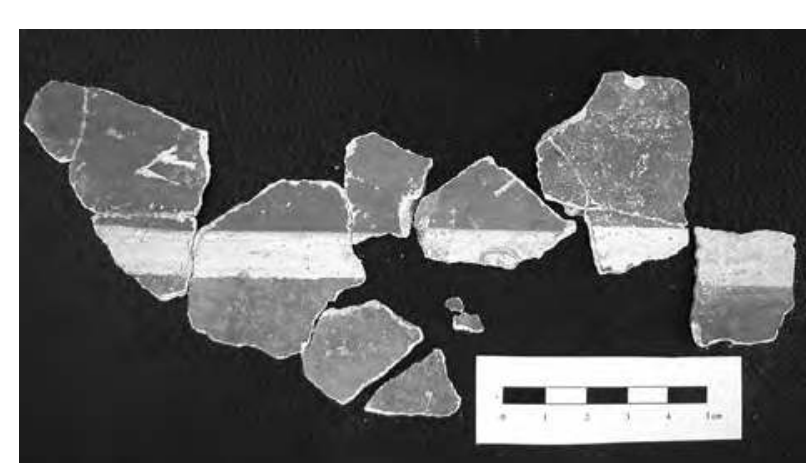

Fig. 5.9.1. : Fragments from the East Church. The early decoration phase (A. Freccero).

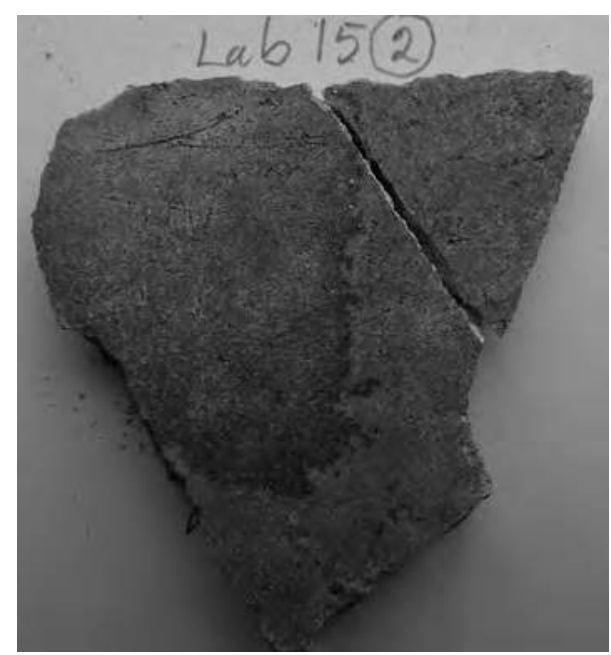

Fig. 5.9.3. : Fragment Lab 15-2. (A. Freccero).

the Labraunda fragments into a firm chronology of wall decorations in the Mediterranean area.

Many of the structures visible at Labraunda today are from the $4^{\text {th }}$ century BC or later ${ }^{137}$. After the Hekatomnid construction phase there was seemingly a slower building activity in the Hellenistic period, but as large areas of the sanctuary have not yet been fully excavated new finds might prove otherwise. In the Roman era, some great buildings were erected and old ones restored or reconstructed in order to meet new needs ${ }^{138}$. The last building period seems to be dated to the $4^{\text {th }}$ and $5^{\text {th }}$ century AD, as for example in the complexes belonging to the East and West Churches where old structures were reused $^{139}$. The fragments in this investigation pre-

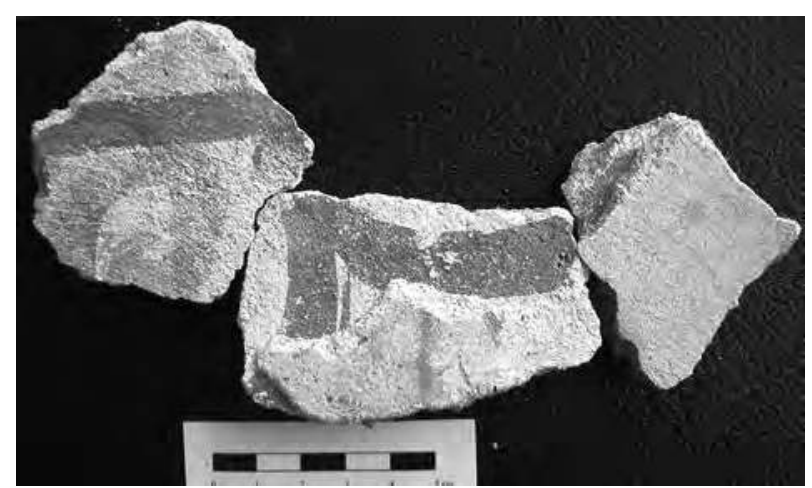

Fig. 5.9.2. : Fragments from the East Church. The last decoration phase (A. Freccero).

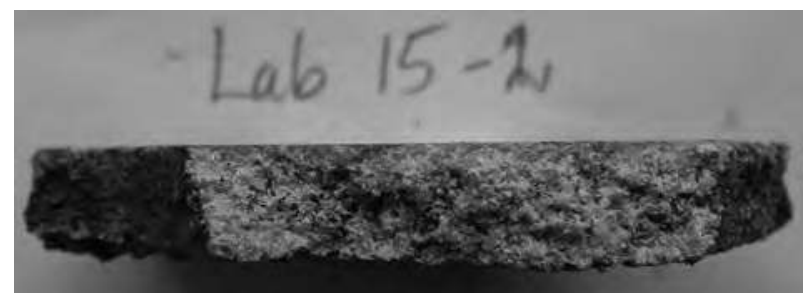

Fig. 5.9.4. : The fragment in profile. (A. Freccero).

sumably represent the three main building periods: the Hekatomnid, the Roman and the Late Antique.

The Andrôn A was possibly on of the earliest building erected within the extensive program designed by Maussollos and Idrieus in the $4^{\text {th }}$ century BC The building was recently excavated and decorated plaster fragments were found during the excavations at the original floor level. They are suspected to have been part of the original decoration of the Hekatomnid period. Not much is known about wall painting materials in Karia, except for an account from Vitruvius concerning the brick walls at the Palace of Halikarnassos, which were covered with stucco so highly polished that they sparkled as glass ${ }^{140}$. The palace was constructed by Mausollos at approximately the same time as the Andrôn A was built by Idrieus ${ }^{141}$.

The East Church stratigraphy is complex: the building was constructed as part of the Roman Bath, which partially incorporated the Doric House of the Hekatomnid period ${ }^{142}$. Among the filling materials from the floor in the apse were fragments of deco-

137) Westholm 1963; Hellström 2007 and 2011

138) Large areas of the sanctuary have not yet been excavated.

139) See Blid 2016.

140) Vitruvius 2.10

141) On the chronology of the andrônes at Labraunda, see the forthcoming volume by P. Hellström in the Labraunda Series.

142) Unpublished report by A. Henry. 
rated plasters (Fig. 5.9.1-2) ${ }^{143}$. It is not known from which room or which building the fragments derive, but a qualified guess would be the Roman Bath, which according to Blid is the earliest example on "Romanization" in Labraunda ${ }^{144}$. Many fragments represent two decoration periods, as evidenced by the fact that one decorated plaster layer was applied upon the earlier decoration. Usually an existing decoration is torn down before re-decoration is made, but that was not made in this case. Ocular inspection of the plasters and painted motifs indicate that the earlier decoration might be dated to the early Roman era, and that the last decoration was made in the late Roman or early Christian period.

\subsubsection{PLASTER INVESTIGATIONS}

Preliminary ocular observations revealed that plaster and stucco at Andrôn A were of high quality, and made by skillful craftsmen. The fragments have much in common with the First Style decorations at Pompeii and Prima Porta and, from a technical point of view, with the materials found at Ossaia and Agios Donatos. The flat and lustrous surfaces are characteristic for the First Style decorations but, since no recessed borders have been found, the decoration may have consisted of a well smoothed, polished and painted surface without the intricate First Style relief design, maybe similar to the Pompeian plain style. Two minor studies of mortars found during the excavation of Andrôn A had been made when I received the Andrôn A fragments. Arnaud Coutelas had analyzed plasters and stucco on seven fragments and concluded that although they were representative for the technique described by Vitruvius it was not possible to assign a specific date for the decoration on the basis of traditional analytical methods ${ }^{145}$. Another study carried out by Duygu Er- genç, concerned the building mortars. Ergenç identified material from 351-344 BC, as well as reparation mortar dated to the Roman era ${ }^{146}$.

The East Church fragments had not been studied before. They were of two types, and in many cases one decoration covered the earlier one ${ }^{147}$. The early decoration technically consists of a basic rough plaster layer, succeeded by finer layers topped with polished and painted stucco ${ }^{148}$. The patterns which remain consist of rectangles resembling variously coloured slabs in relief, which is characteristic of the Second Style decoration, in vogue in Italy roughly between $80-20 \mathrm{BC}$ It is not possible to establish the exact date of the present decoration, but they might be connected with the Roman bath, which was constructed around 50 AD It might therefore be assumed that the fragments are remains of a decoration in the first building phase of the Roman Bath, and that the room was later redecorated in a rougher manner. The late decoration consisted of rapidly painted decorative elements such as flowers and garlands on a rough plaster with no stucco layer, a characteristic of decorations from the late antiquity and onwards.

\subsubsection{ANALYTICAL LABORATORY EXAMINATIONS}

Analytical laboratory examinations of the materials reveal that there are material and technical differences between these fragments, as well as to those in previous studies ${ }^{149}$. To begin with, the Andrôn A fragments were of high quality, but had some unusual features. They contained only calcite crystals and no marble in the stucco, which is the most common but not mandatory feature. The paint was applied directly on to the smooth marmorino without any layer of lime or extra-fine stucco in be-

143) Blid 2016: 157.

144) Blid 2016: 142.

145) Coutelas 2015: 4.

146) Ergenç 2015: 306.

147) 20 fragments had two recognizable decoration periods.

148) 198 fragments were cleaned and studied.

149) The analytical methods applied were as follows. The samples were analyzed with XRF by putting the painted surface in contact with the head of the portable instrument (Bruker TRACER III-SD with Rh anode). The samples were then embedded in resin and cut perpendicular to the stratigraphy in order to obtain both cross and thin sections. Cross sections were documented with a stereomicroscope Zeiss, Stemi 200 C equipped with high resolution camera in $1 \mathrm{X}$ to $2 \mathrm{X}$ magnification. Cross sections were documented with an optical microscope Nikon Eclipse $600 \mathrm{C}$ equipped with high resolution camera and software NIS Element. The illuminations used were visible light and UV light for fluorescence images (Block filter Nikon B2A), in magnification ranging from 4X to 10X. Thin sections were observed in polarized transmitted light both with crossed $(\mathrm{X})$, and parallel Nicols $(/ /)$, in magnification ranging from 2,5X to 40X. The microscope used was AxioScope A.1, Zeiss camera with high resolution and software Axiovision for the evaluation of morphometrical characteristics of materials. Cross sections were observed and analysed with Scanning Electron Microscope with EDS (SEM-EDS). Different signals were produced and recorded. The secondary electrons signal (SE) gives information about the surface morphology. The backscattered electrons (BS allow seeing light and heavy elements thanks to a black and white images. Then the characteristic X-Rays, produced by fluorescence phenomenon were recorded to do the elemental analysis. The instrument used was a Quanta FEI ESEM, Philips. 


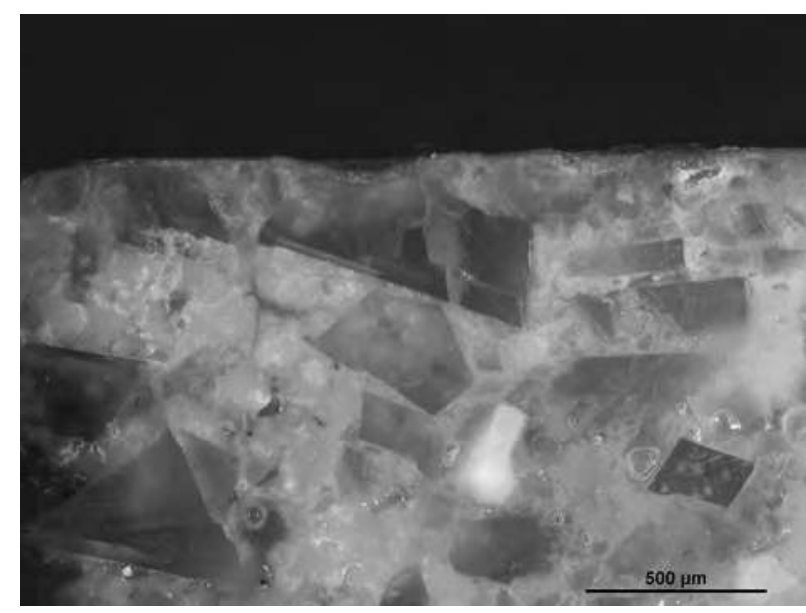

Fig. 5.9.5. : Section of the sample (E. Cantisani, CNR).

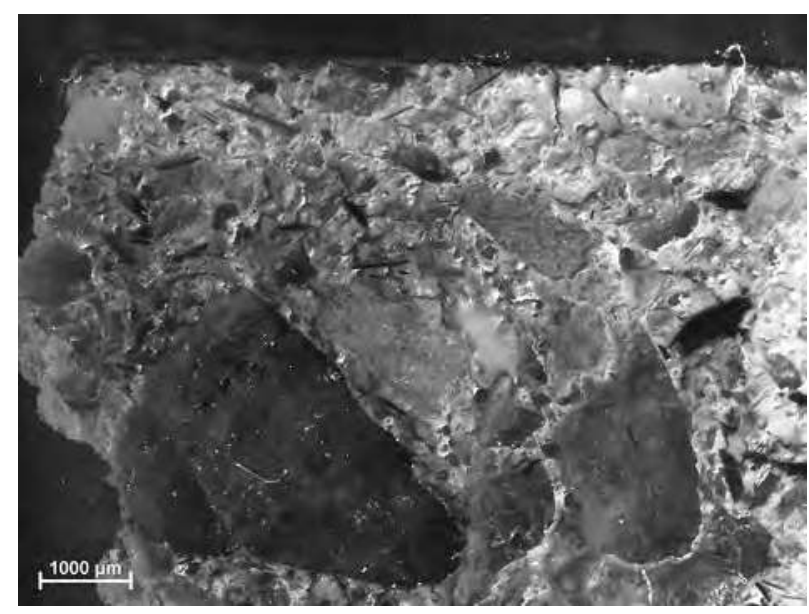

Fig. 5.9.7. : Section of sample Labrom 1 in reflected light. Plaster and stucco (E. Cantisani, CNR).

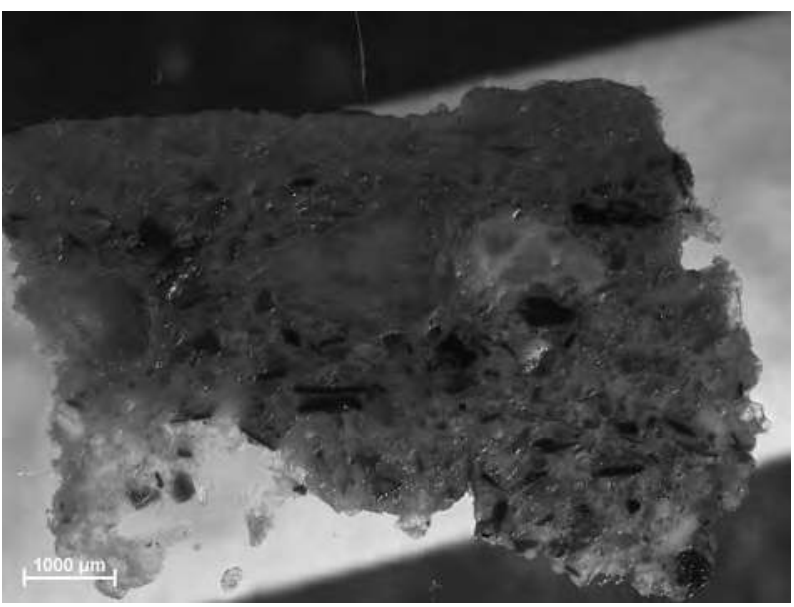

Fig. 5.9.9. : Sample Labrom2. Plaster and paint layer (E. Cantisani, CNR).

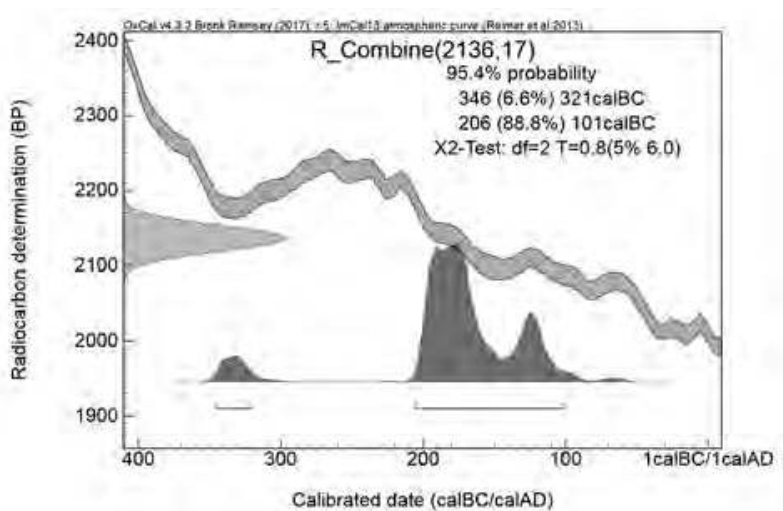

Fig. 5.9.6. : Results of $C_{14}$ analysis. Graph by Alf Lindroos, Åbo University.

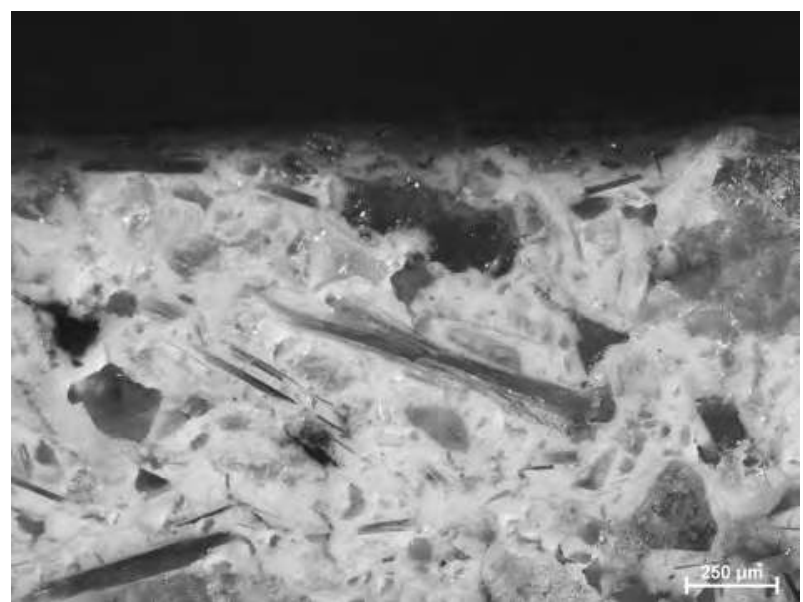

Fig. 5.9.8. : Section of sample Labrom 1 in reflected light. Stucco and paint layer (E. Cantisani, CNR).

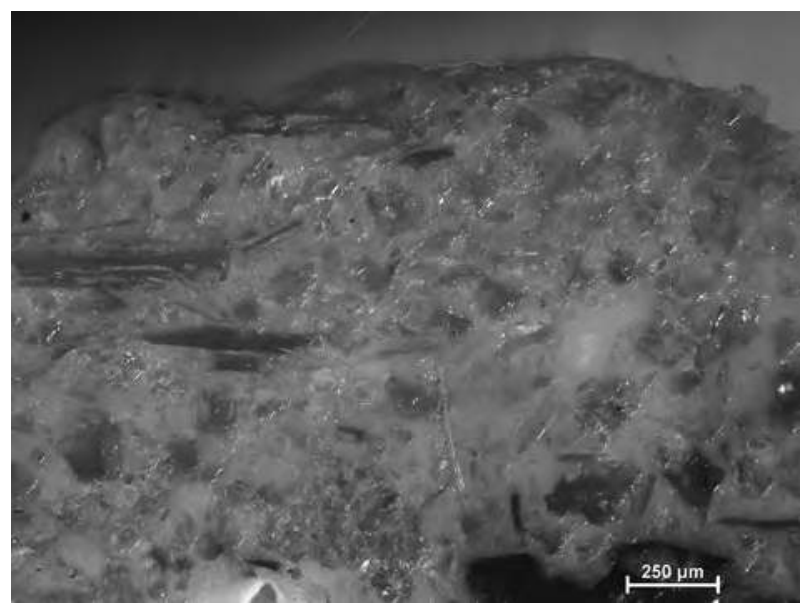

Fig. 5.9.10. : Section of sample Labrom2. Paint layer and paint (E. Cantisan, CNR). 
tween, which is quite unusual, but it explains the crystalline look of the surface in areas where no paint remains (Fig. 5.9.3-5). Analyses of the paints show that the yellow and red pigments were ochre, and the black was carbon, which are, by far, the most common pigments. The painting technique was al fresco, with the addition of paint applied $a$ secco, which was the most common method in antiquity. It was not possible to determine when this decoration was made by means of the methods used so far, because there was no available contemporary material from Asia Minor to which the Andrôn A fragments could be compared.

A more precise dating might be established by $\mathrm{C}_{14}$ analysis. Samples were removed from two fragments, and prepared for such analysis by Alf Lindroos at Åbo University, and thereafter sent to the AMS laboratory at Aarhus University for the $\mathrm{C}^{14}$ analyses. A fragment of the floor mortar was sent for the same analysis. The results show that the plasters and the mortar with highest probability can be dated to 200-150 BC, even though there is a small probability for an earlier date (Fig. 5.9.6). Therefore, the plasters most probably can be dated to the Hellenistic period, when the floor was repaired and the wall redecorated. This is also supported by the technology used for wall plastering and painting.
Two samples of each decoration type from the East Church were analyzed. The objective was to study whether or not materials and the technology changed over time. The result was quite clear: the East Church and the Andrôn A fragments are materially, technologically and stylistically quite different. There is a strong material resemblance between the two periods at East Church, because the filler contains the same kinds of local stone. Technically, there is a difference between the periods: the craftsmanship is of higher quality as far as the early period is concerned and there is no stucco or fine preparation as paint layer on the late decoration (Fig. 5.9.7-10).

Summing up, ocular observations as well as laboratory analyses show a difference between the Hellenistic, the Roman and the Late Antique technology used for wall decoration. The Hellenistic decoration in Andrôn A represents the supreme materials and technique, the early Roman decoration is quite well made and has most refined surface, and the late antique decoration has a comparatively rough and low-class standard. The results obtained at Labraunda are compatible with the results achieved in my earlier studies of wall decoration, in which materials dated from the $3^{\text {rd }}$ century $\mathrm{BC}$ to around $300 \mathrm{AD}$ have been investigated.

\section{FOUILLES}

\subsection{PRESS CLOSE TO THE BUILT TOMB} (by A. Sitz) ${ }^{150}$

Excavations were carried out at two olive presses in the area of the Built Tomb (sometimes referred to as the Monumental Tomb) in order to determine the extent and date of these agricultural and economic installations at the edge of the ancient sanctuary of Zeus Labraundos. Although the territory controlled by the temple was involved in agricultural production already in the Hellenistic period, as attested by the letters of the Olympichos dossier (dated ca. 240220 BC, I.Labraunda \#1-7, \#137), the site's raison d'être at some point shifted from a religious center to an economic one, presumably after the decline or prohibition of pagan cult in late antiquity $\left(4^{\text {th }}\right.$ to $7^{\text {th }}$ century $\mathrm{AD}$ ). The precise date for this change in site usage between late antiquity and the Middle Byzantine era $\left(9^{\text {th }}\right.$ to $12^{\text {th }}$ century AD), however, as well as the full extent of agricultural production around the ancient site, are poorly understood. We therefore seek to better understand the two olive presses near the Built Tomb, while concurrent work at Labraunda by Christophe Bost is shedding light on presses located to the west of the sanctuary.

The Built Tomb stands on a terrace (Built Tomb Terrace $\mathrm{C},=\mathrm{BTC}$ ) on the slope of the mountain to the north, between the sanctuary and the acropolis fortification further up the mountain (Fig. 6.1.1). The large tomb is constructed of ashlars and belonged to an important person, perhaps a member of the Hekatomnid family. In order to clarify the construction date and original appearance of this impressive terrace visually rising above Temple of

150) I wish to thank the Kolb Society of Fellows at the University of Pennsylvania Museum of Archaeology and Anthropology for a Greenewalt project grant that allowed the excavation of the presses to be carried out. The report was written with the support of the Joint Fellowship of the Deutsches Archäologisches Institut and the Harvard Center for Hellenic Studies. I would also like to thank the site director, Olivier Henry, for his assistance with the excavation, Christophe Bost for his helpful suggestions, Vasilica Lungu for her ongoing analysis of the ceramics, Frédérique Marchand-Beaulieu for photo documentation, and my fellow excavators, Friederike Kranig and Çağla Durak. 
Zeus lower in the sanctuary, previous excavations by Olivier Henry and Mélissa Cormier on the eastern part of the terrace revealed a large, unroofed $\mathrm{Pi}$-structure (so called because of its shape) in front of the Built Tomb; this likely was used as an openair setting for the commemoration of the occupant of the tomb (Fig. 6.1.2) ${ }^{151}$. An even earlier, pre-Hekatomnid room with a threshold was also found underneath the Pi-structure at the eastern edge of the terrace, representing one of the earliest structures at Labraunda. The construction date of the Pi-structure and its chronology in relation to the Built Tomb terrace as a whole remained unclear from these previous excavations.

\subsubsection{BTC EXCAVATION}

The work of the 2017 season on the BTC extended the previous trench of Henry and Cormier to the west in order to complete the investigation of the terrace as a whole. The BTC 2017 sector was divided into two parts, Zone 1 to the east and Zone 2 to the west (Fig. 6.1.3). The delineation between these two zones was determined by the western wall of the Pi-structure, running north to south. In Zone 2, which is heavily sloped from a large rock outcrop to the north towards the terrace wall to the south, we encountered unworked bedrock almost immediately after cleaning the topsoil, which ranged from $0.05-0.15 \mathrm{~m}$ in depth. This section of the Built Tomb Terrace $C$ therefore seems to have been largely unused in antiquity. Few sherds were collected from Zone 2.

Construction and occupation activity on the Built Tomb Terrace $\mathrm{C}$ was rather concentrated to the east, in Zone 1. As mentioned above, the several layers of alluvial runoff in the area had already been removed by Henry and Cormier, revealing a sizeable (1.10 m diameter) press stone with a maximum elevation of $694.58 \mathrm{~m}$ asl. A partially collapsed wall, designated BTC 17 Wall 1 (founded on bedrock at $694.75 \mathrm{~m}$ asl), was visible in the northern part of Zone 1, built abutting the rear (north) wall of the Pi-structure. Fallen stones, ranging in size from 0.10 to $1.00 \mathrm{~m}$ and mostly unworked, were visible on the surface throughout the sector when the work of 2017 commenced. This layer of fallen stones, most likely representing the collapse of Wall 1 , contained a large amount of ceramic material, including a number of well-preserved profiles.

In the northwestern part of Zone 1, a segment of floor paved with smooth, irregularly-shaped stones was found underneath the fallen stone layer (Fig. 6.1.4-5). Between this floor and the round press stone, a large re-used ashlar was set into a ground of packed soil and seems to have functioned as a grinding stone, based on the shallow groove on its upper face. Removing the soil around the round press stone revealed that it is sitting on a rectangular base of worked stones. To the east of the press, a large $(1.50 \mathrm{~m}$ diameter) pit was found underneath the layer of fallen stones (Fig. 6.1.6). The pit likely originally held a pithos to catch the oil from the press. A significant quantity of sherds was found within the pit, including fragments possibly from a pithos or other storage vessel. The spout of the round press stone was also found broken inside this pit on top of soil and sherds, indicating that the pit and press had fallen out of use before the damage to the press stone. It is expected that the analysis of the sherds from the pit will provide some indication of the date of the press installation; a date in the late Roman period (or earlier) seems most likely based on the lack of glazed Byzantine ceramics in this year's work on the BTC.

In any case, the press seems to be the latest feature of the Built Tomb Terrace $\mathrm{C}$ in our investigation. After the press and surrounding areas had fallen out of use, the entire area was sealed underneath fallen stones, likely originating from Wall 1, as mentioned above, and perhaps the collapse of a north-south wall built on top of the Pi-west wall. Installing the press involved digging down on the terrace to the Hellenistic/late classical levels (the fill of the terrace wall), thereby destroying any intervening strata or any surface or paving that had originally covered the space within the Pi-structure during the original use phase of the Built Tomb. In the eastern half of Zone 1, a pressed earth surface (694.10 $\mathrm{m}$ asl), likely anthropogenic and perhaps associated with the press, lies immediately above a layer of homogenous fill (sandy whitish soil with large and small stone inclusions, $c a .0 .10-0.50 \mathrm{~m}$, and occasional tiles). This is presumably the original fill of the Pi-structure and terrace. This fill of sandy soil, stones, and tiles reaches the uneven, unworked bedrock at around $693.4 \mathrm{~m}$ asl.

Our excavations furthermore confirmed that the $\mathrm{Pi}$-structure is contemporary with the construction of the terrace wall and presumably the Built Tomb itself. The west wall of the Pi-structure is bonded with the terrace wall. The bedrock was cut down to the west in order to build this Pi-structure. Immediately behind (north) of the terrace wall, we en- 


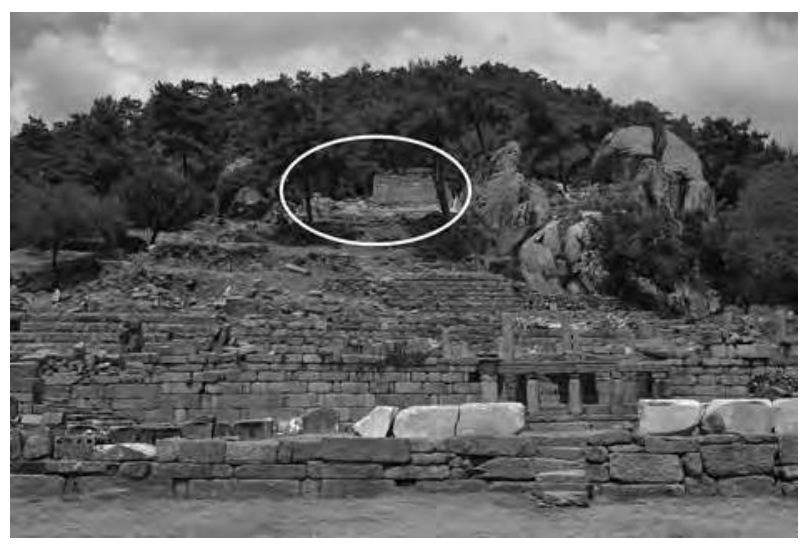

Fig. 6.1.1. : View of the sanctuary from below. Monumental Tomb and terrace circled (A. Sitz).

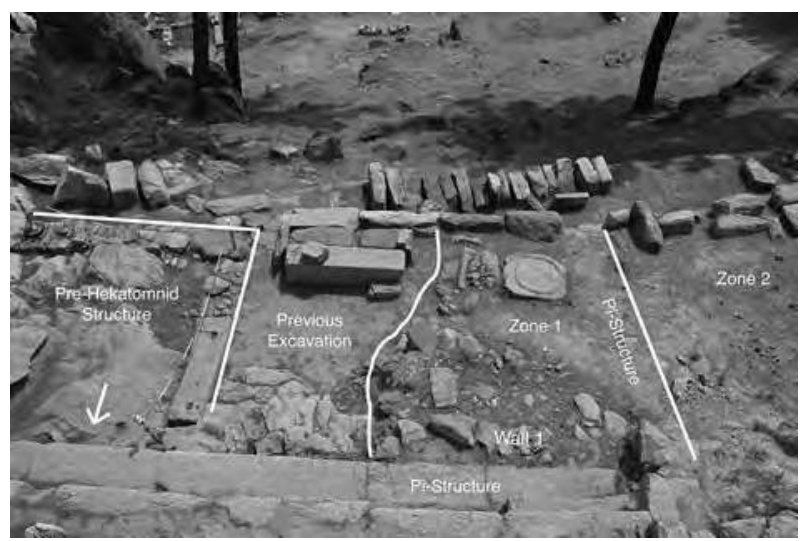

Fig. 6.1.3. : The BTC area before the 2017 season (A. Sitz).

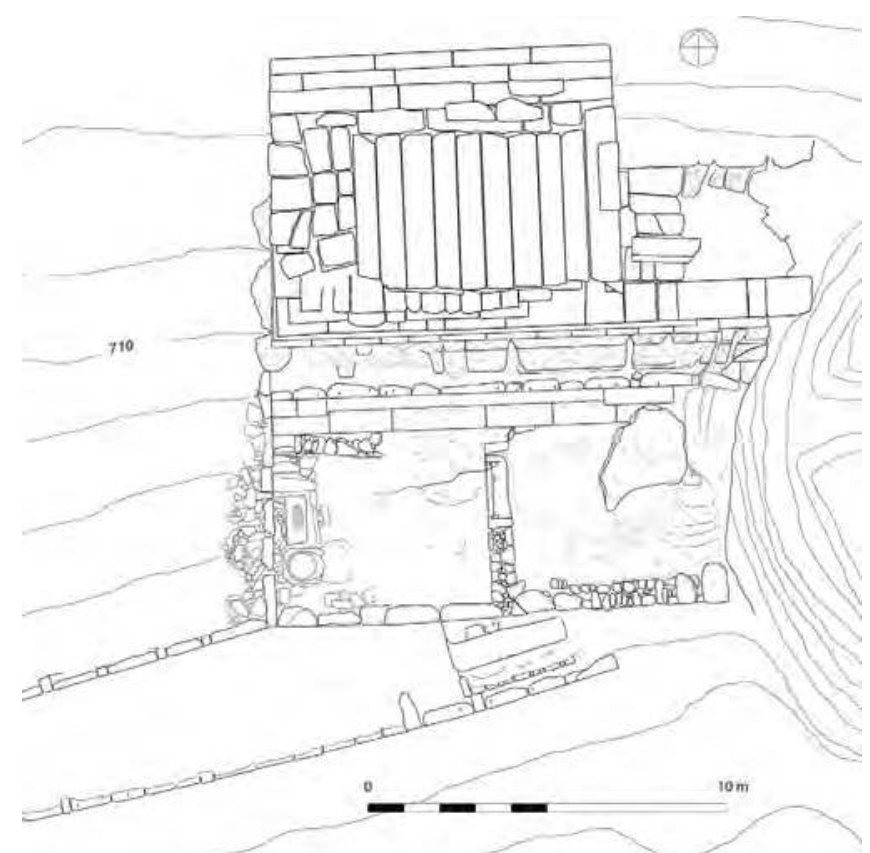

Fig. 6.1.4. : General plan of BTC after the 2017 season (O. Henry and A. Sitz).

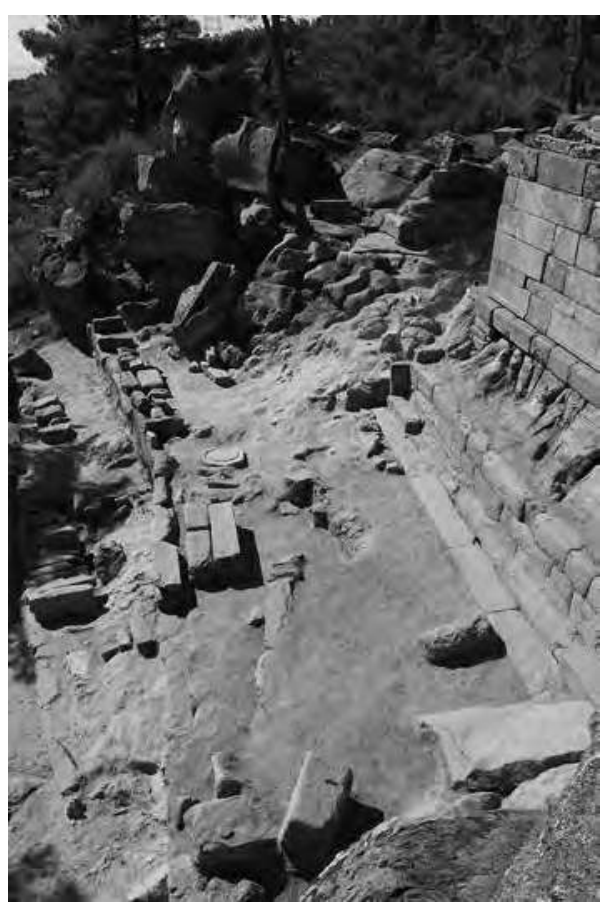

Fig. 6.1.2. : View of BTC from the East after the 2012 excavation season (L. Karlsson).

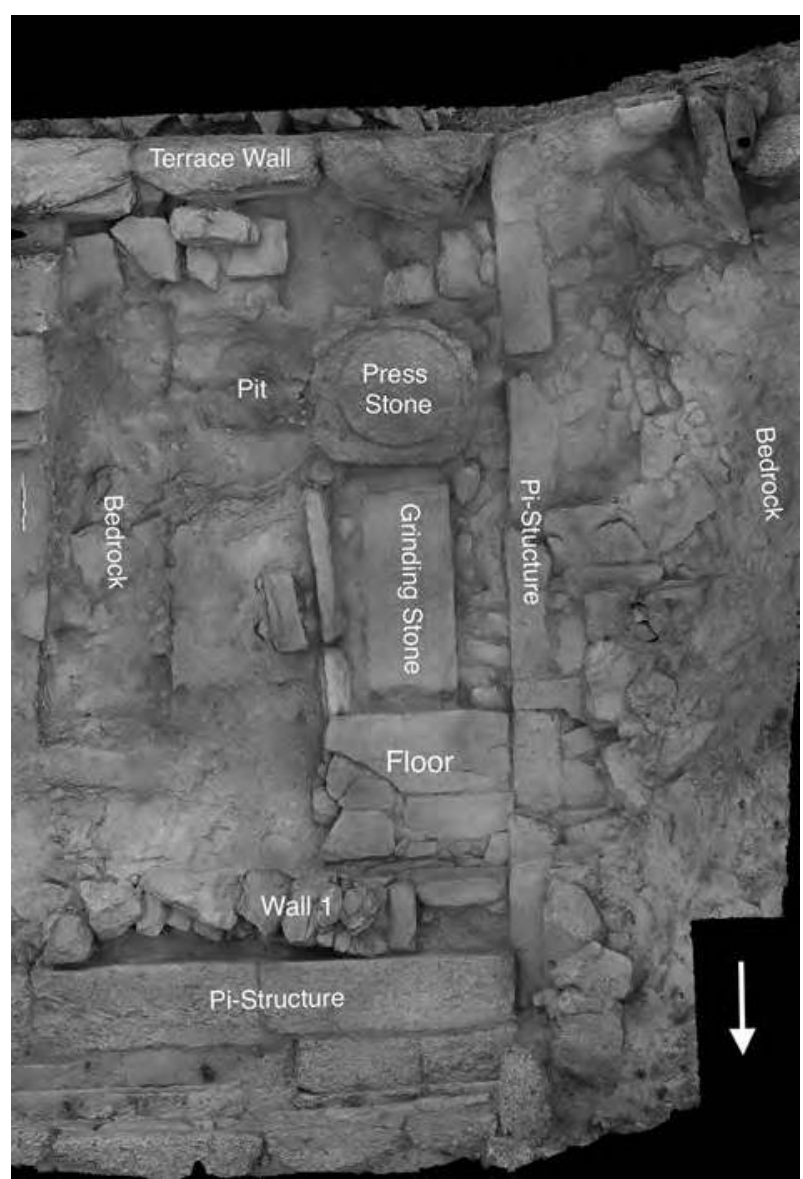

Fig. 6.1.5. : Ortho-photo of Zone 1 after excavation (Fr. Marchand, with labels added). 


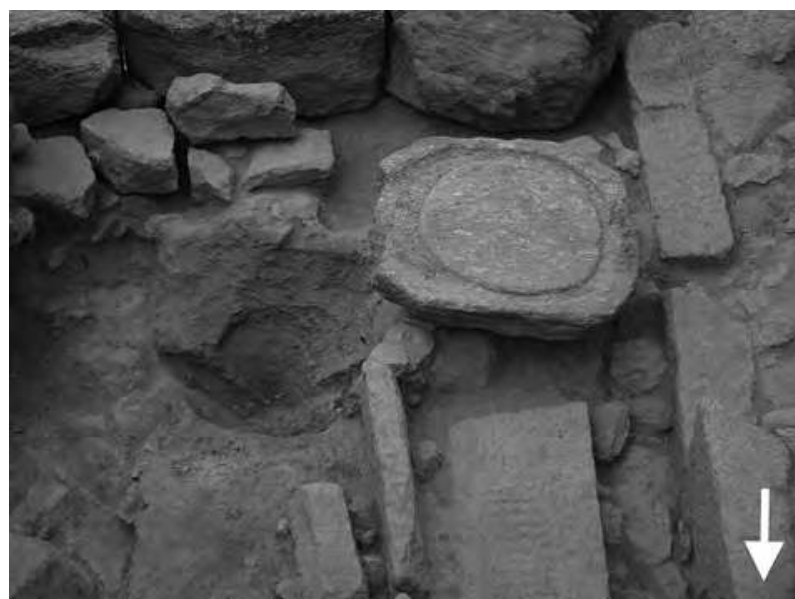

Fig. 6.1.6. : Round press stone, pit, and grinding stone after excavation (A. Sitz)

countered several large $(c a .0 .60 \mathrm{~m})$ roughly worked stones; these likely represent an attempt to stabilize the terrace wall installed during the original construction and at the same time as the sandy whitish fill mentioned above. At the end of the season, we partially deconstructed Wall 1 in order to remove unstable stones, leaving the lowest courses in place. We then back-filled the eastern half of Zone 1, leaving exposed the round press stone, grinding stone, and floor because of their scientific and touristic interest.

\subsubsection{BTP EXCAVATION}

About twenty meters to the west of the Built Tomb, higher up on the slope above the BTC, we investigated the surroundings of a rock-cut press (at an elevation of $707.84 \mathrm{~m}$ asl) discovered on the surface during cleaning in the previous season but never previously documented. This area is designated as the Built Tomb Press (BTP) sector. We removed the topsoil around this small press $(0.48 \mathrm{~m}$ diameter); the limits of our excavation were determined by the protrusions of bedrock circumscribing a level area in front of the press. Removing the topsoil (ca. 0.10-0.30 m) revealed uneven, unworked bedrock throughout the sector. A limited number of sherds and charcoal were found, but it is unclear whether these represent human activity in the area or were alluvial deposits originating higher on the slope of the mountain.

Immediately in front of the small press, we uncovered a cavity in the bedrock approximately 0.60 $\mathrm{m}$ in depth, which likely functioned as a pit for a $p i$ thos or other storage container to receive the oil produced by the press (Fig. 6.1.7). Although no sherds

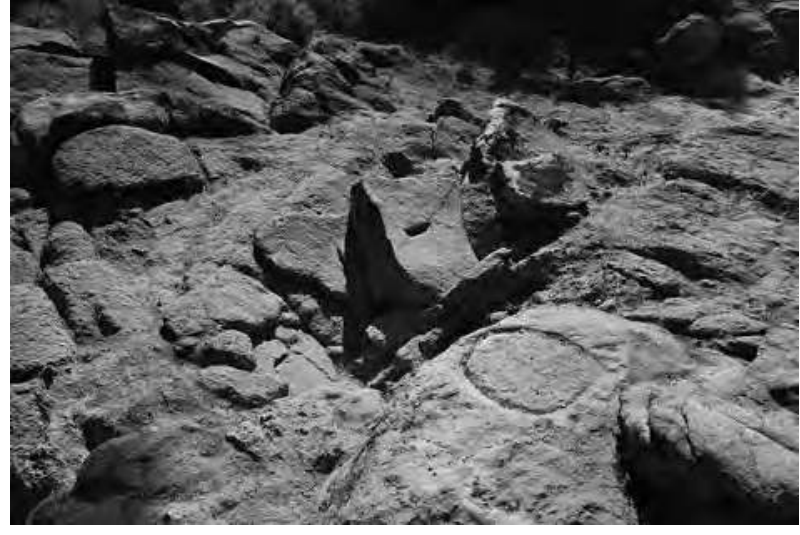

Fig. 6.1.7. : BTP, after excavation. Rock-cut press and cavity (A. Sitz)

from such a storage vessel were found in situ, several other sherds and a coin were found within the cavity; when analyzed, they may provide data for the use of the press. A cutting in the rock next to the cavity may be related in some way to the use of the press, but its precise purpose is unclear. No additional evidence for the press installation was found, and, based on its small size, it is likely that it functioned for private production, rather than larger scale economic activity.

\subsubsection{SUMMARY}

The 2017 season in the area of the Built Tomb therefore confirmed that the open-aired Pi-structure in front of the Built Tomb was built concurrently with the late classical/Hellenistic terrace wall. It also clarified the contexts of the two later press stones in the area. The BTP to the west of the Built Tomb was revealed to be a small, solitary installation, while that on the tomb terrace (BTC) was part of a larger set-up including a grinding stone and pit. A space originally intended for the commemoration of deceased elites buried in the Built Tomb became at some point a place of mundane agricultural production, still taking place in the shadow of the Built Tomb. Located at the edge of the late classical sanctuary of Zeus, the economic activity on the Built Tomb Terrace $\mathrm{C}$ attests to the continued cultivation of olive trees at the site, known from at least the Hellenistic period, but the processing of these olives was most likely taking place closer and more visibly than previously to the sanctuary's monumental center. At present we cannot precisely date the end of pagan worship at Labraunda, nor determine whether the BTC press was installed before or after the Christianization of the site, but future analysis 


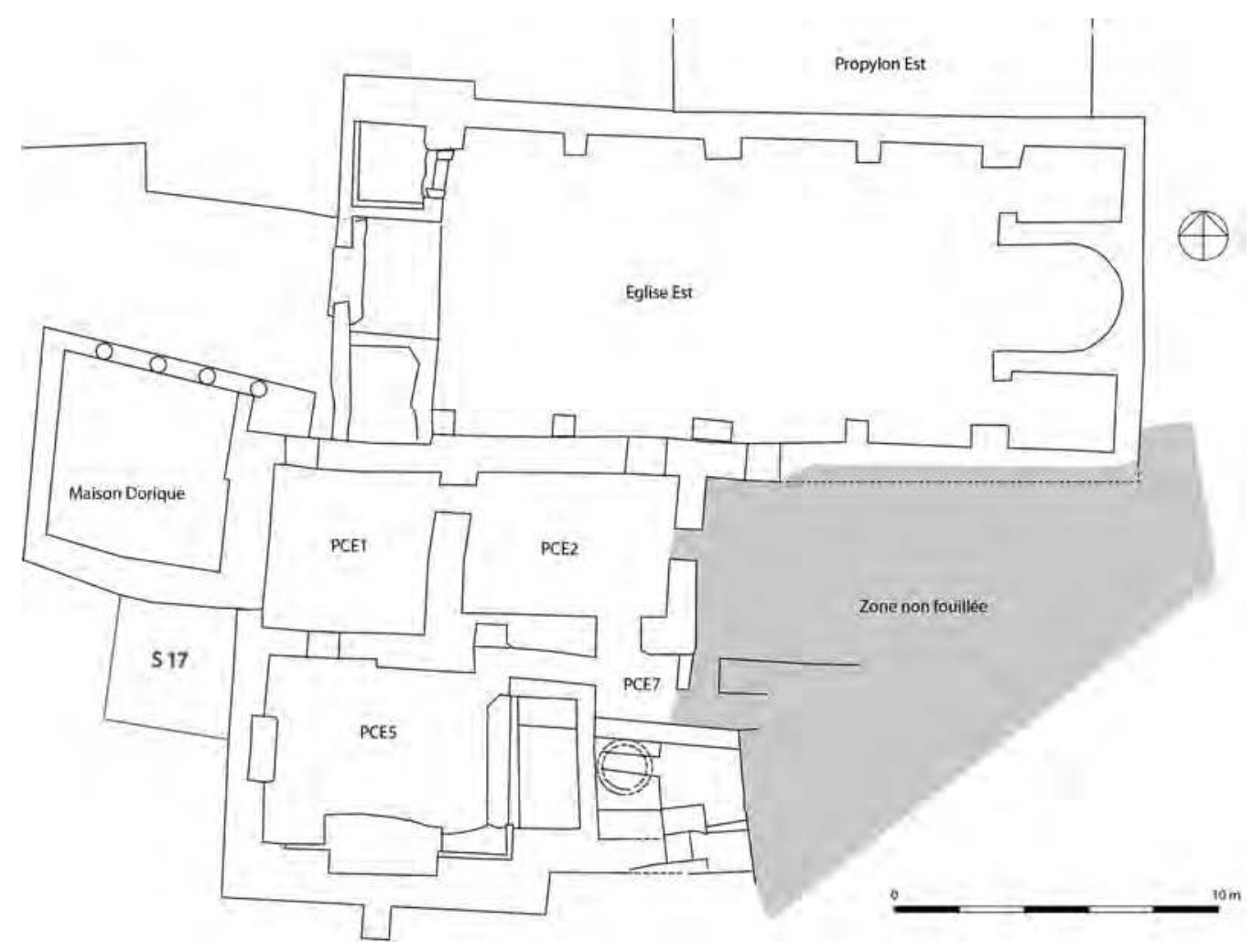

Fig. 6.2.1. : Situation du sondage S17 (N. Lamare).

of the ceramics should shed more light on the date of each of these features.

\subsection{LES BAINS EST}

Au cours de l'année 2017, le travail de l'équipe de recherches des East Baths a suivi trois directions principales. Il s'agissait d'abord de poursuivre les études des différentes catégories de mobiliers archéologiques exhumés au cours des campagnes précédentes (citons les lots de céramique, TCA, verre, les petits objets métalliques, les restes de faune et les prélèvements de sédiments). Ce choix de porter l'effort vers les études de mobilier s'imposait. En effet, les importants dégagements de plusieurs salles des bains, entrepris durant les étés 2014, 2015 et $2016^{152}$, avaient donné lieu à la mise au jour d'une quantité importante d'artefacts qu'il convenait d'étudier intégralement, non seulement afin de progresser dans la compréhension que l'on peut avoir de l'histoire du complexe, mais également pour faire face aux problèmes de stockage sur place, que pose immanquablement une telle masse de ma- tériaux. De concert, il semblait important d'achever les relevés d'élévations, compléter leur description et poursuivre l'étude architecturale. Enfin, une fois précisées ces priorités, s'imposait alors le principe d'une fouille qui devait rester modeste, pour cette année du moins. En ce sens, la décision fut prise d'ouvrir un unique sondage, référencé S17 et implanté à l'angle que forme le mur ouest des Bains avec le mur sud de la Doric House (Fig. 6.2.1).

\subsubsection{LA FOUILLE DU SONDAGE S17 $7^{153}$ (par Chr. Bost)}

\section{a. Objectifs et méthodes}

En 2016, les opérations de dégagement des façades sud et ouest du caldarium avaient révélé le parement extérieur du mur (Fig. 6.2.2). Ce dernier présentait des assises conservées appartenant à un type d'appareil remarquable, certes bien connu à Labraunda, mais jusque-là toujours associé à des édifices d'époque hécatomnide ou, pour les plus récents, hellénistique. En effet, cette maçonnerie,

152) Voir à ce sujet, les comptes-rendus publiés dans Anatolia Antiqua, Henry et al. 2015 : 355-366; $2016: 424-435$ et 2017 : 245-255.

153) Le travail de terrain a, en partie, été conduit par Mélanie Hauchart (doctorante, Université Paul Valéry-Montpellier 3) avec l'aide d'Anne-Sophie Laurent et Anıl Arslan. 
caractérisée par un grand appareil à carreaux et boutisses avec plumées d'angle, est en tous points similaire à celles mises en œuvre sur les grands murs de terrasse comme sur ceux de l'acropole, dont on connaît mieux aujourd'hui la datation, grâce aux récentes recherches menées par B. Vergnaud ${ }^{154}$. Par déduction, on supposa alors que les murs ouest et sud des Bains étaient venus s'implanter, en totalité ou en partie, sur une base appartenant à un édifice bien antérieur. C'était là une découverte pleine d'intérêt qui ne manquait pas de poser de nouvelles questions. Est-il possible de dater la construction de ce premier bâtiment et de préciser son rapport avec la Doric House ? Pouvons-nous situer la phase de reconstruction et de transformation en édifice balnéaire ? En choisissant d'implanter un sondage à l'extérieur des Bains, au pied du mur ouest et au Sud de la Doric House, on pensait se donner les moyens d'apporter des éléments de réponse.

Conformément au modèle mis en place dès 2014, la fouille a été conduite suivant des méthodes canoniques, soit un enregistrement stratigraphique par Us (Unité stratigraphique) avec levée photographique et couverture altimétrique systématiques par niveau. Les listes d'Us, photos et relevés sont dressées au fur et à mesure de l'avancée et l'ensemble du mobilier archéologique découvert est soigneusement récolté, enregistré et conditionné. En outre, les archéologues responsables tiennent à jour un cahier de notes. Enfin, bénéficiant de l'appui et l'expertise de F. Marchand-Beaulieu (CNRS-AOrOc), nous avons tenté de mettre en place un protocole de levées photogrammétriques avec, comme finalité, la création d'un modèle 3D qui restituerait les principaux états identifiés tout au long de la fouille.

Les limites du sondage formaient arbitrairement un carré de 4 mètres de côté. Dans un premier temps, sur plus de $1,70 \mathrm{~m}$ de profondeur, la fouille s'est développée dans le respect de l'enchaînement stratigraphique sur toute l'emprise, avant que le travail ne se concentre sur deux secteurs plus réduits, situés au pied de chacun des deux murs.

\section{b. Premiers résultats de l'analyse stratigraphique}

En l'état actuel, le travail d'analyse est loin d'être achevé, car il manque, entre autres, l'étude céramique complète comme la mise au propre définitive de l'information graphique, coupes et plans, toutes choses qui sont en cours. Cependant, en s'appuyant sur l'ordonnancement stratigraphique, il est d'ores et déjà possible de distinguer plusieurs contextes que l'on présentera succinctement.

Les travaux de décapage ont débuté à une altitude supérieure de $662,75 \mathrm{~m}$. Il était d'abord question d'évacuer des déblais de fouilles anciennes, provenant sans doute des Propylées, de la Doric House ou des Bains et mêlant un amas de blocs de maçonneries sur plus de $0,50 \mathrm{~m}$ d'épaisseur (Fig. 6.2.3). Les premiers niveaux archéologiques en place ont été atteints à une altitude de $662,10 \mathrm{~m}$.

\section{- Contexte 1: Us 10 003, 10013.}

Principalement composé de blocs de gneiss de tailles très variées, allant du fragment non taillé de quelques centimètres aux blocs d'architecture de grand module, cet ensemble disparate s'apparente à un remblai. Au Sud du sondage, quatre blocs posés de chant semblent alignés et marquer de la sorte une limite (Fig. 6.2.4)

- Contexte 2 : Us 10 004, 10005,10006 , $10007,10008,10009,10012,10016,10017$.

Une fois enlevées ces strates de remblais difficiles à dater, le niveau archéologique suivant est apparu très différent, se composant d'une succession de couches de sédiments. Une première Us sombre recouvrait un niveau de terre limoneuse indurée, armé de gravier et parsemé de nombreux tessons de céramiques souvent très fragmentées. Situé à une altitude de $661,70 \mathrm{~m}, \mathrm{l}$ 'ensemble de ces indices nous invite à l'interpréter comme un niveau de circulation et d'occupation (Us 10 006) (Fig. 6.2.5). Au Sud, les blocs de chant semblent bordurer ce sol qui venait prendre appui contre trois dalles posées à plat. Au Nord et à l'Est, au pied du mur de la Doric House comme de celui des Bains (MR 6007), apparaissait le sommet de deux zones empierrées. Appuyée contre le mur MR6007, une colonne monolithe en gneiss (longueur $1,20 \mathrm{~m}$, diamètre $0,44 \mathrm{~m}$ ), posée à l'horizontal sur ces niveaux, formait part d'un aménagement en lien avec l'occupation (Fig. 6.2.6).

- Contexte 3 : Us $10018,10019,10020$, $10021,10022,10023,10025,10028$.

Mises au jour à des altitudes comprises entre $661,20 \mathrm{~m}$ et $661,07 \mathrm{~m}$, plusieurs structures signalent une phase d'occupation antérieure. On observe la base d'un mur (MR 10 019) axé Nord-Sud, qui ne partage pas l'orientation du mur des Bains. D'une longueur observée de $3 \mathrm{~m}$ pour une largeur de $0,70 \mathrm{~m}$, il est conservé sur une hauteur de deux assises de blocs de gneiss à peine dégrossis (Fig. 6.2.7).

154) Voir, entre autres, le compte rendu de B. Vergnaud dans Henry et al. $2016: 397-412$ et les conclusions du même chercheur sur les questions de datation de la forteresse de l'acropole dans Henry et al. $2017: 215-220$. 


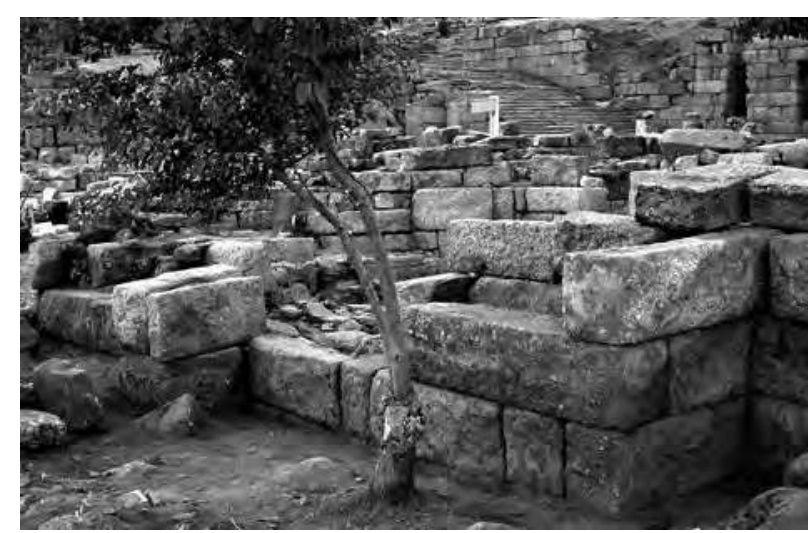

Fig. 6.2.2. : Parement de la façade sud du caldarium (Chr. Bost).

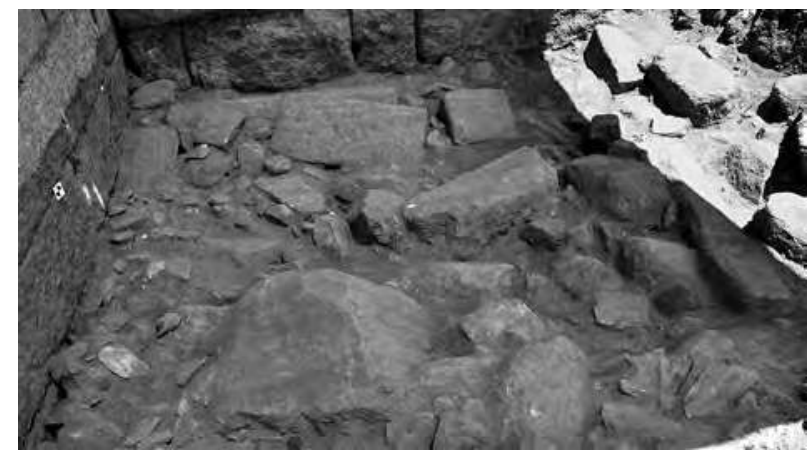

Fig. 6.2.4. : Niveau de remblai du contexte 1 (Chr. Bost).

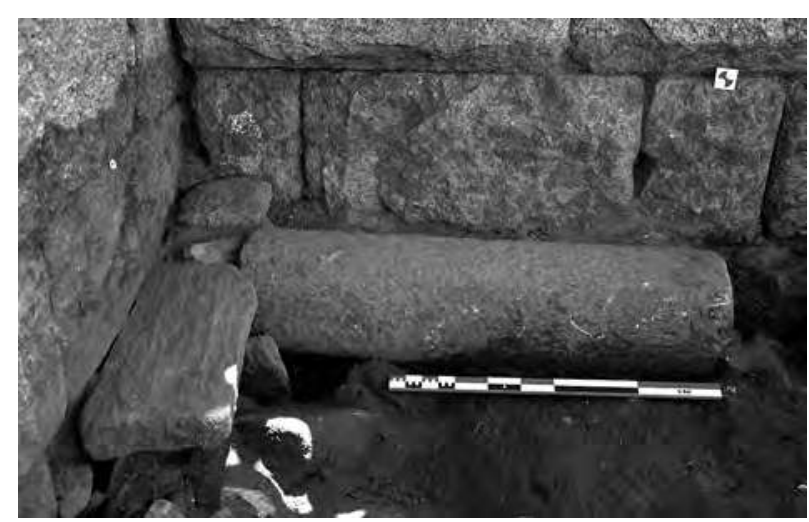

Fig. 6.2.6. : Colonne appuyée contre le mur des bains (Chr. Bost).

A l'Ouest de ce mur, s'ouvre une fosse d'environ $0,25 \mathrm{~m}$ de profondeur, comblée par des pierres et des fragments de tuiles (FS 10 021). Enfin, contre la berme ouest, on a délimité une poche de terre sombre charbonneuse circonscrite par un liseré orangé marquant une rubéfaction et témoignant sans

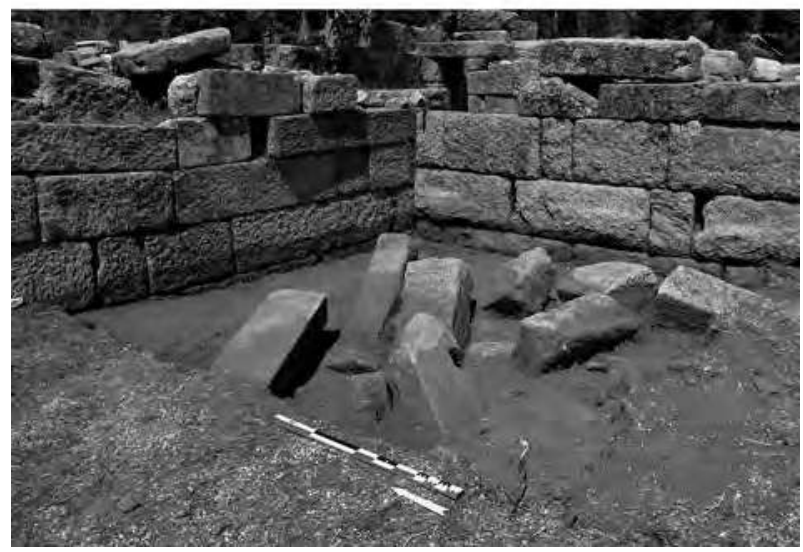

Fig. 6.2.3. : Déblais de fouilles anciennes

(Chr. Bost).

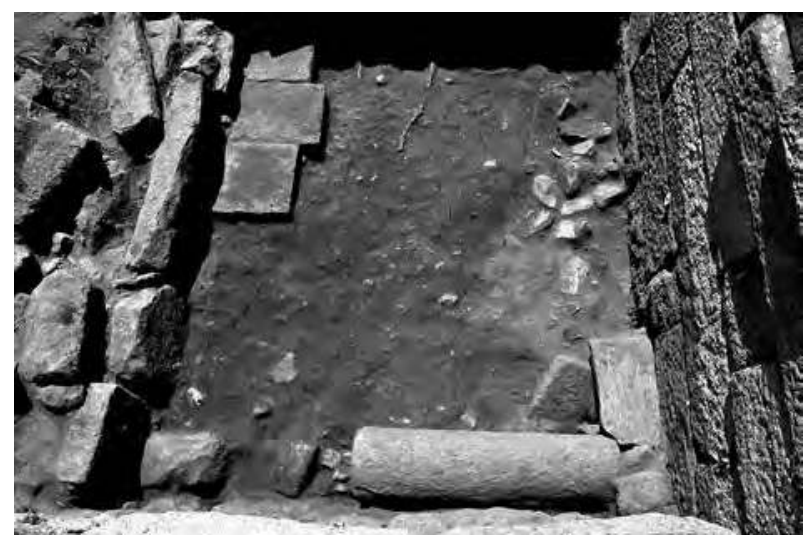

Fig. 6.2.5. : Niveau d'occupation du contexte 2

(Chr. Bost).

doute de l'emplacement d'un foyer (Us 10 022) (Fig. 6.2.8). Avant d'aller plus loin, il importe de bien noter qu'en raison des limites imposées par une fenêtre d'observation somme toute réduite et sans liaison concrète avérée, il est, pour l'heure, impossible d'associer avec certitude ce mur à ces quelques traces d'occupation (foyer et fosse).

- Contexte 4 : Us 10 029, 10 030, 10031 , $10032,10033$.

A compter de la côte d'altitude de $661 \mathrm{~m}$, nous avons choisi d'ouvrir deux sondages profonds plus limités, l'un contre le mur de la Doric House, l'autre, contre celui des Bains (Fig. 6.2.9). Ce dernier, d'une longueur de $1,90 \mathrm{~m}$ pour une largeur de $0,90 \mathrm{~m}$, nous a permis d'observer la tranchée de fondation du mur des Bains. Le mur de fondation est maçonné sans empattement, l'étroit espace entre le mur et la paroi de la tranchée a été comblé par apport de terre sombre comportant des fragments de gneiss (Us 10 029) (Fig. 6.2.10). Quelques précieux tessons de 
céramique à vernis noir ont été exhumés au sein de ce comblement. A l'altitude de $660 \mathrm{~m}$, dans la partie sud du sondage, la tranchée de fondation est venue couper un niveau charbonneux d'une vingtaine de centimètres d'épaisseur (Us 10 033) (Fig. 6.2.11). Cette couche posée sur le substrat contenait des tessons et des restes de faune témoignant d'une occupation bien antérieure à l'édification des deux murs. Exhumée dans le second sondage, la fondation de la Doric House semble obéir aux mêmes principes de tranchée étroite, bien que la délimitation apparaisse moins nettement.

\section{c. Premiers éléments de réponses}

- Un niveau de circulation et des aménagements en lien avec le début des Bains

Le contexte 2 est caractérisé par un sol de circulation et des aménagements le long des murs (Fig. 6.2.5). Celui positionné contre la Doric House se compose d'un empierrement et peut être interprété comme un drain ayant pour fonction d'assainir la base du parement. Au pied du mur des bains (MR 6007), il s'agit plus d'une tranchée dont le fond est pavé d'éclats de gneiss, liés au mortier. Les parois, quant à elles, sont pour partie stabilisées par des assises de briques (Fig. 6.2.12). Vers le Sud, c'est la base du mur ancien MR 10019 qui est réemployé pour faire office de bordure. Pour l'heure, il est difficile d'attribuer une fonction précise à ce qui devait être une canalisation. Sur ce point, la réflexion doit se poursuivre mais nous proposons comme hypothèse de relier cet aménagement aux Bains.

En effet, s'agissant du problème de datation de ce contexte, si l'étude de l'important lot de mobilier est en cours, les premiers éléments à notre disposition, certes encore trop imprécis, nous orientent vers le $1^{\text {er }}$ s. ap. J.-C. A ce point, il faut revenir sur la date communément retenue pour l'édification des Bains, celle proposée par l'épigraphiste J. Crampas dans son travail de publication des inscriptions de Labraunda. Ainsi, les exemplaires 20 et 65 du catalogue, évoquant la dédicace des bains par Tiberius Claudius Menelaus, sont datés du milieu du $1^{\mathrm{er}} \mathrm{s}$. ap. J.-C. ${ }^{155}$ (Fig. 6.2.13). Dès lors, il y a de bonnes raisons de relier cette occupation à la phase de remaniement correspondant à la construction des Bains. Les résultats de l'étude céramique conduite par Alina Musat-Streinu, avec l'aide de Vasilica Lungu, devraient offrir de précieuses informations complémentaires sur une période qui, jusque-là, n'avait pas été documentée par la fouille.
- Sous les Bains : un bâtiment construit entre le début du $4^{e}$ s. et la fin du $3^{e}$ s. av. J.-C.?

Les observations tirées des sondages profonds implantés aux pieds des murs méritent attention. Ici encore, l'étude du lot de céramique provenant du comblement de la tranchée de fondation du mur MR6007 devrait permettre de proposer une datation bien étayée. Les premières observations de fragments attiques à vernis noir pointent le $4^{\mathrm{e}} \mathrm{s}$. av. J.-C.

De la sorte, au fil des découvertes, c'est un nouvel édifice qui semble se dessiner (Fig. 6.2.14). Les murs ouest et sud apparaissent distinctement, réemployés à la base des parements de façades du caldarium. S'agissant du mur nord, sa fondation est conservée dans les soubassements de l'hypocauste de la salle PCE1. D'autre part, si l'on poursuit son tracé vers l'Est, alors l'élévation du mur sud du tepidarium PCE2 (MR 6005) se trouve parfaitement alignée et l'on peut supposer qu'elle est bâtie sur cette même fondation ancienne. En l'état de nos connaissances, nous n'avons en revanche aucune trace permettant de proposer une restitution pour la partie est de l'édifice.

Qu'en est-il de la relation entre ce nouvel édifice et la Doric House ? Pour avancer sur ce problème, il faut sans doute observer attentivement le point de contact entre l'extrémité sud-est de la Doric House, dont il ne reste que le soubassement conservé dans le sol de l'hypocauste de la salle PCE1, et le mur nord du bâtiment (Fig. 6.2.15). Ici, il semble bien que le mur nord soit venu buter contre la fondation de la Doric House. De ce point de contact jusqu'à l'angle avec le mur ouest, la fondation du mur nord s'est trouvée plus étroite de quelques centimètres, contrainte par les superstructures de la Doric House. Ainsi, si nous retenons ce scénario de chronologie relative, ce nouveau bâtiment serait venu s'appuyer contre la Doric House et serait par conséquent postérieur. Que savons-nous de la date de construction de la Doric House ? La dédicace restituée sur l'architrave du monument mentionne Idrius, dont on sait qu'il succède à son frère Mausole, comme dynaste de Carie, de 351 à 344 av. J.-C. Somme toute, en attendant les conclusions de l'étude céramique, nous tenons là un terminus post quem pour ce nouveau bâtiment.

Pour conclure, il convient une nouvelle fois de souligner qu'il s'agit là d'un ensemble d'hypothèses sur lesquelles nous travaillons actuellement. Toutefois, il est certain que ces premiers résultats ne peuvent que nous encourager à poursuivre la réflexion. 


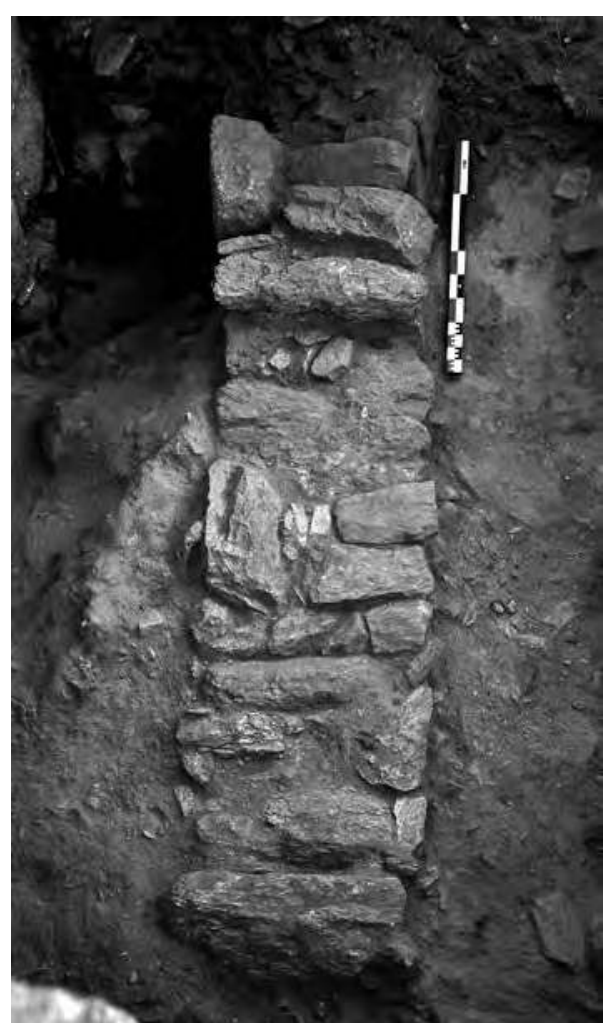

Fig. 6.2.7. : Mur MR 10 019, contexte 3 (Chr. Bost).

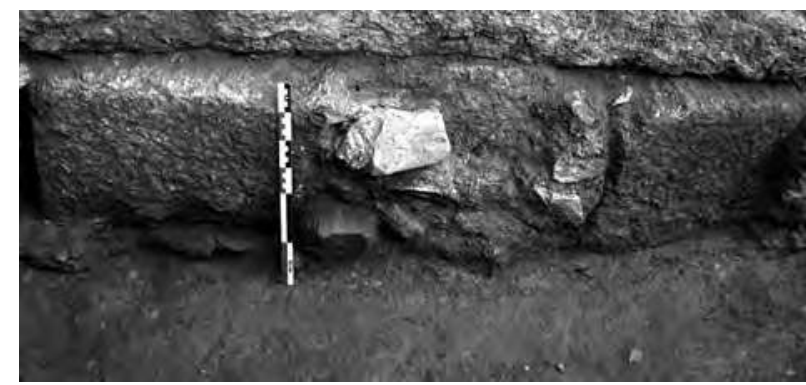

Fig. 6.2.10. : Tranchée de fondation étroite du mur MR 6007, comblée par du sédiment sombre (Chr. Bost).

\subsubsection{ETUDE ARCHITECTURALE DES BAINS EST (par N. Lamare)}

L'objectif de la campagne 2017 était de documenter l'ensemble de PCE8 mise au jour en 2016 et identifiée à un praefurnium (Fig. 6.2.16). Un relevé pierre-à-pierre en plan de la totalité des structures au $1 / 20^{\text {e }}$ (Fig. 6.2.17) et un autre du mur périphérique nord au $1 / 50^{\mathrm{e}}$ ont été dressés et complèteront le plan d'ensemble de l'édifice. De plus, deux élévations ont été dressées au $1 / 20^{\mathrm{e}}$, l'une Ouest-Est (MR6031) qui met en évidence l'un des montants du conduit de chauffe du praefurnium et la porte d'accès à un corridor de

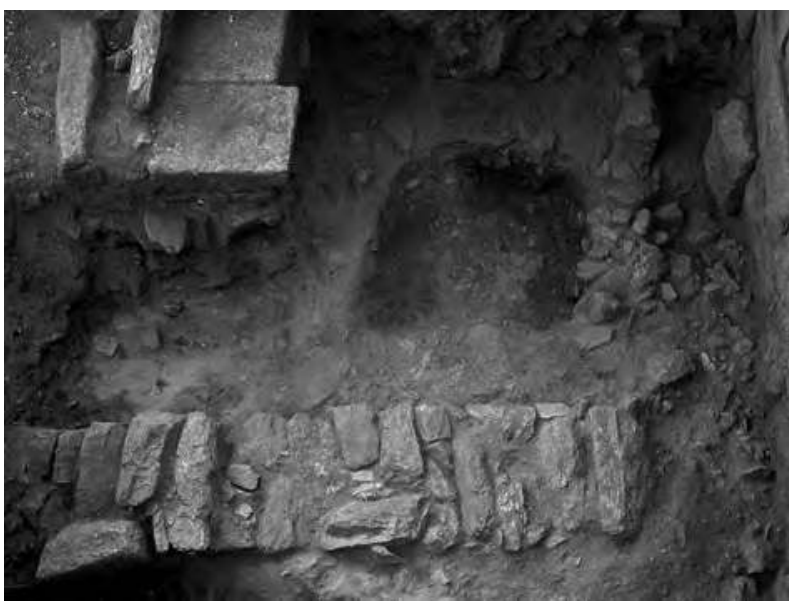

Fig. 6.2.8. : Mur MR 10 019, fosse et traces de foyer, contexte 3 (Chr. Bost).
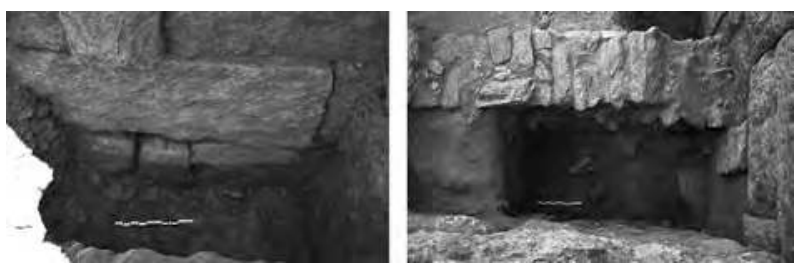

Fig. 6.2.9. : Sondages profonds, fondations du mur sud de la Doric House et du mur ouest des bains (MR 6007) (Chr. Bost).

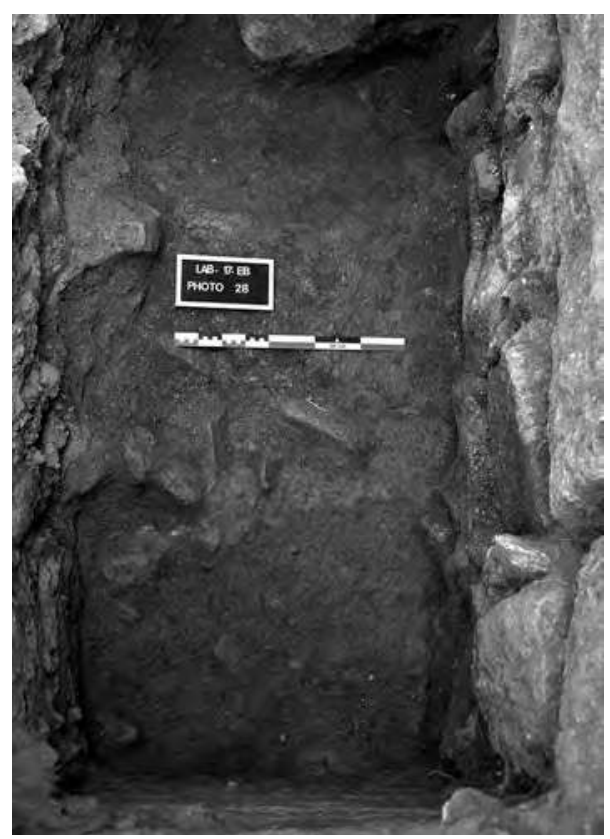

Fig. 6.2.11. : US 10 033. Niveau charbonneux recoupé par la tranchée de fondation du mur MR 6007 (Chr. Bost). 


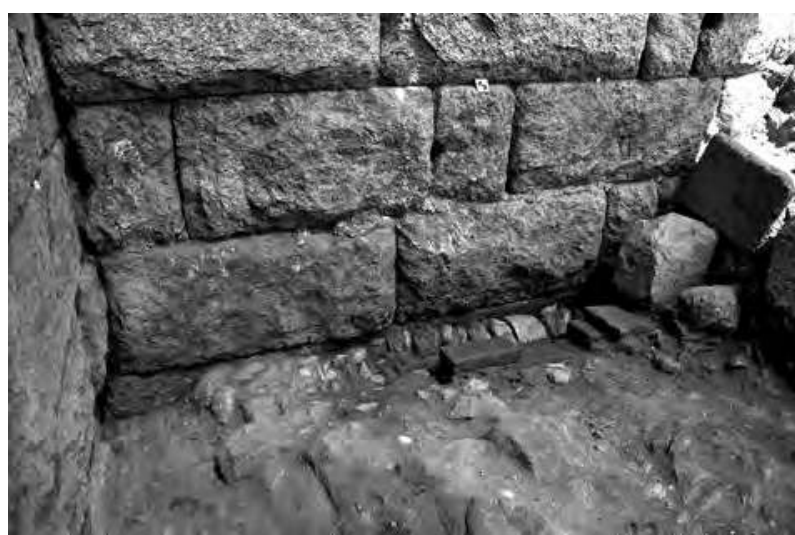

Fig. 6.2.12. : Aménagement au pied du mur MR 6007, contexte 2 (Chr. Bost).

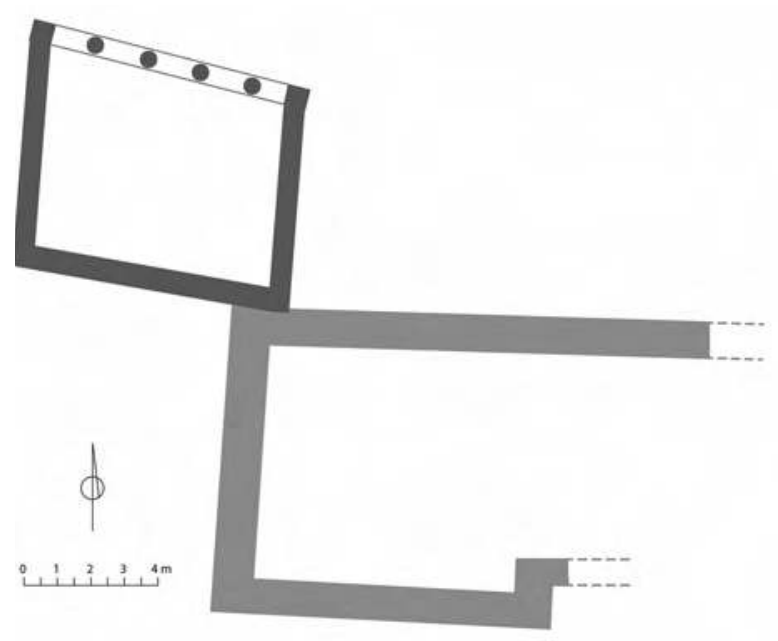

Fig. 6.2.14. : Plan schématique du bâtiment accolé à la Doric House, début $4^{\mathrm{e}} \mathrm{s}$. - fin $3^{\mathrm{e}} \mathrm{s}$. av. J.-C. (N. Lamare).

service (Fig. 6.2.18), l'autre Sud-Nord qui représente la structure en escalier de la zone de service et l'arcade conservée du conduit de chauffe qui ouvrait sur l'hypocauste de PCE5 (Fig. 6.2.19). Ces deux élévations permettront de compléter d'une part la section générale Ouest-Est des bains PCE5-PCE8 et de dessiner la section Sud-Nord à travers PCE8-PCE7-PCE2. Quelques relevés complémentaires des murs périmétraux ouest et sud, recouverts par la végétation et dégagés à la fin de la dernière campagne, ont été pratiqués. Par ailleurs, un sondage a été ouvert à l'extérieur des bains, dans l'angle formé avec la Doric House. Les deux élévations mises au jour jusqu'aux fondations ont été relevées au $1 / 20^{\mathrm{e}}$, le mur sud de la Doric House et le mur ouest des bains. Les coupes stratigraphiques de l'ensemble du sondage et des sondages complémentaires à l'aplomb des murs

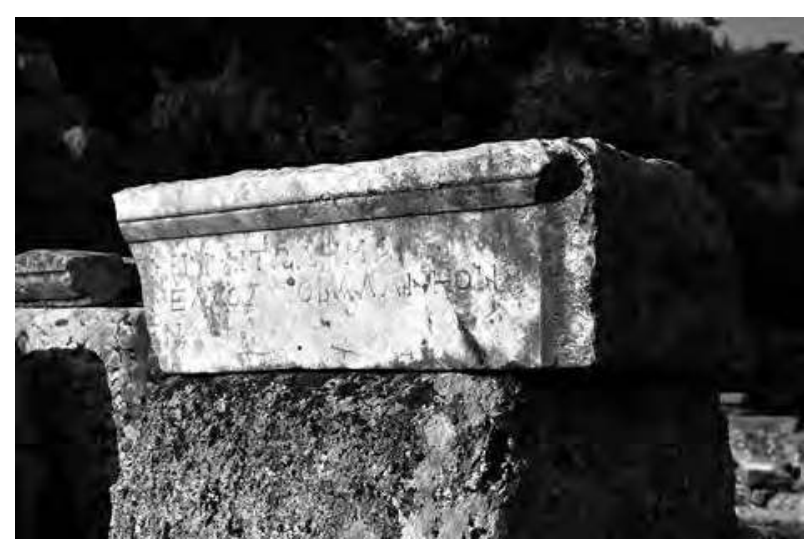

Fig. 6.2.13. : Inscription 20 posée sur un mur de façade de l'église est (Chr. Bost).

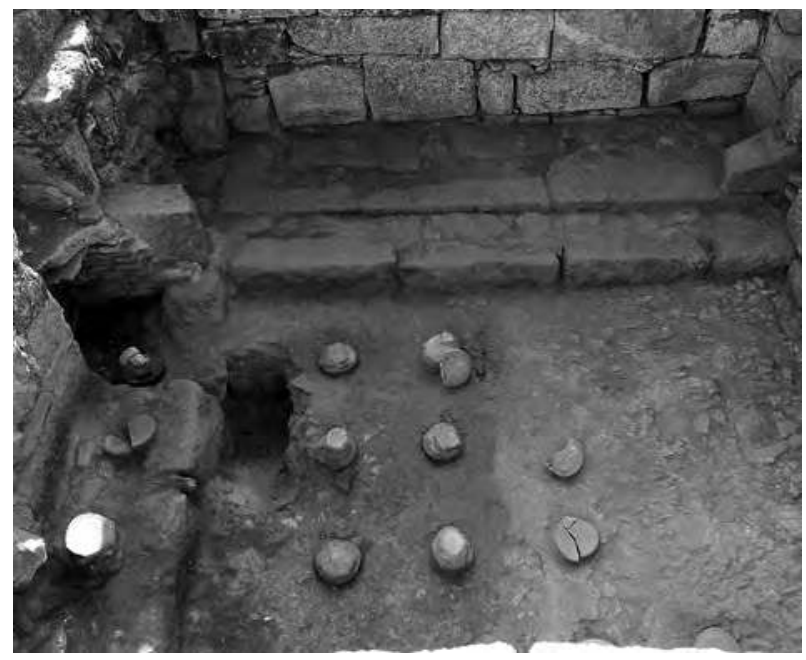

fig 6.2.15. : PCE 1, soubassement du $1^{\text {er }}$ état de la Doric House et vestige de fondation du mur nord (Chr. Bost).

ont été dessinées. Deux plans schématiques des structures découvertes dans le sondage ont été levés. Enfin, les colonnes et blocs architecturaux remarquables mis au jour lors de la fouille ont été dessinés au $1 / 10^{\mathrm{e}}$.

Le tableau (Fig. 6.2.20) présente la description de l'ensemble des murs des Bains mis au jour jusqu'à présent. Il est possible de distinguer plusieurs types d'aménagements qu'il conviendra d'identifier et de dater :

- Grand appareil à double parement de blocs de gneiss aménagés en carreaux et boutisses, dont la largeur est identique pour plusieurs tronçons de murs $(6001,6002,6005,6007,6015)$ qui pourraient appartenir à une même phase d'aménagement.

- Appareil mixte employant à la fois des blocs, peut-être en remploi, et des moellons ou des briques. Les murs qui délimitent le bassin de PCE5 sont ca- 


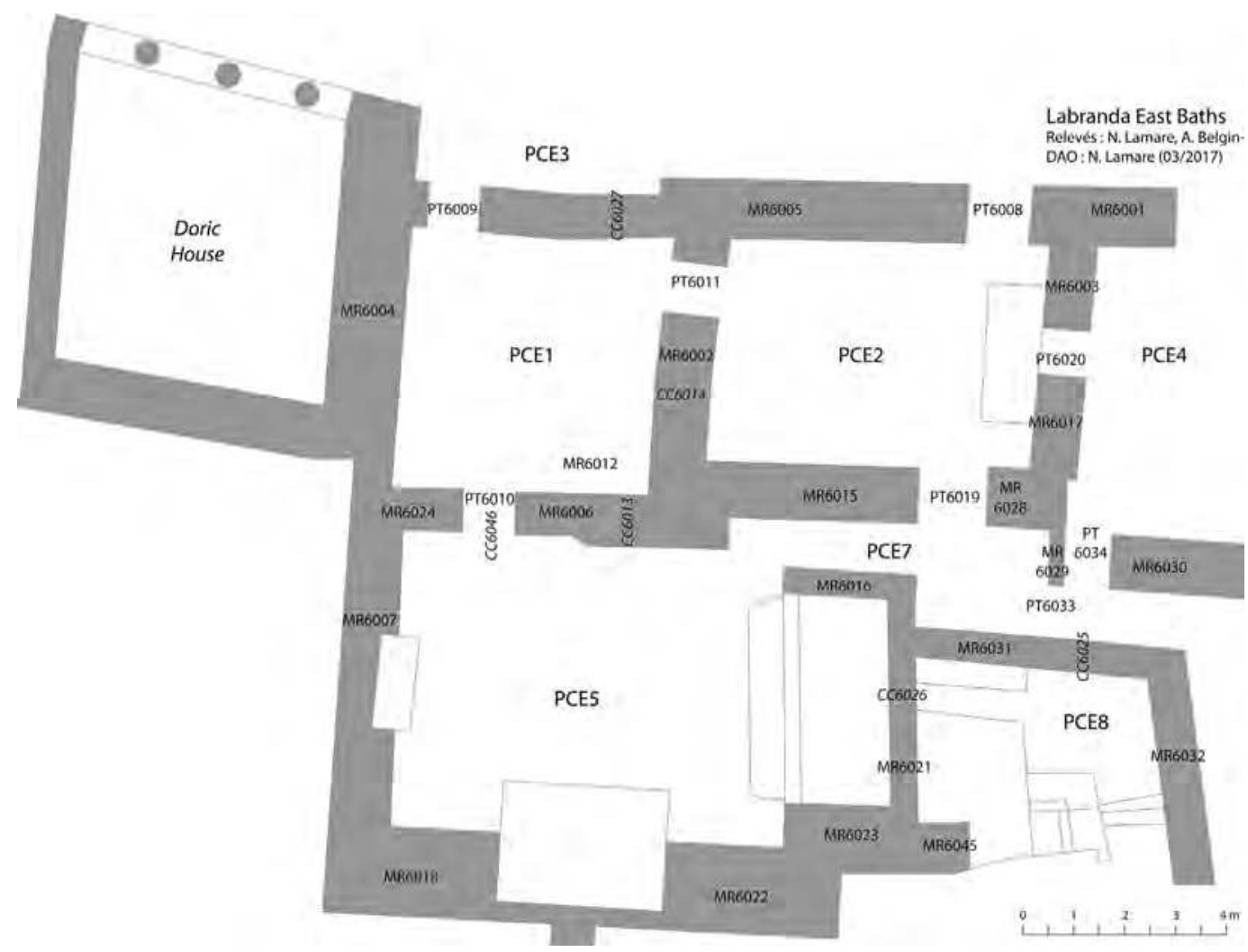

Fig. 6.2.16. : Plan schématique général des structures fouillées des bains est (N. Lamare).

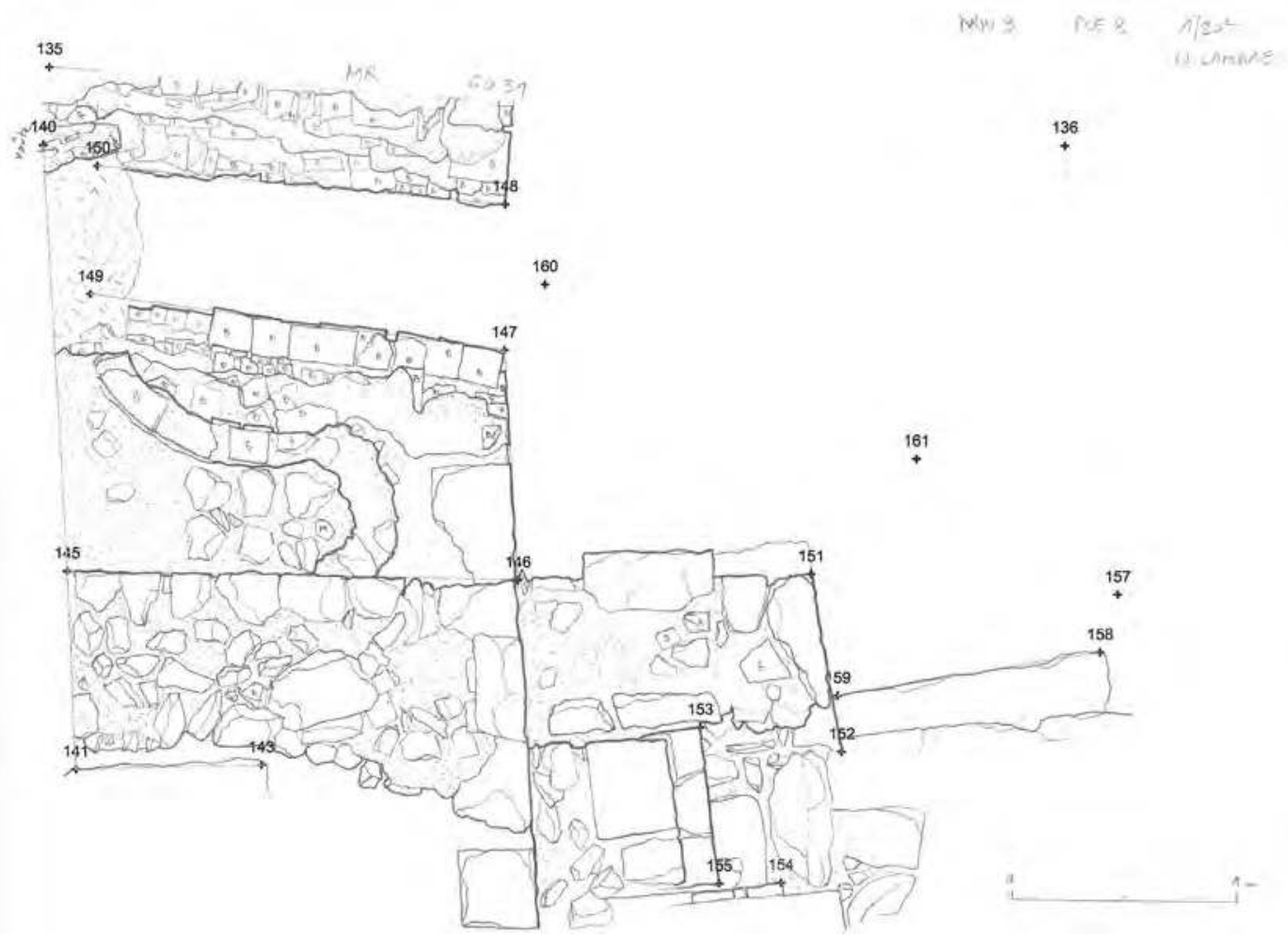

Fig. 6.2.17. : PCE8, praefurnium, relevé pierre-à-pierre en plan (N. Lamare). 


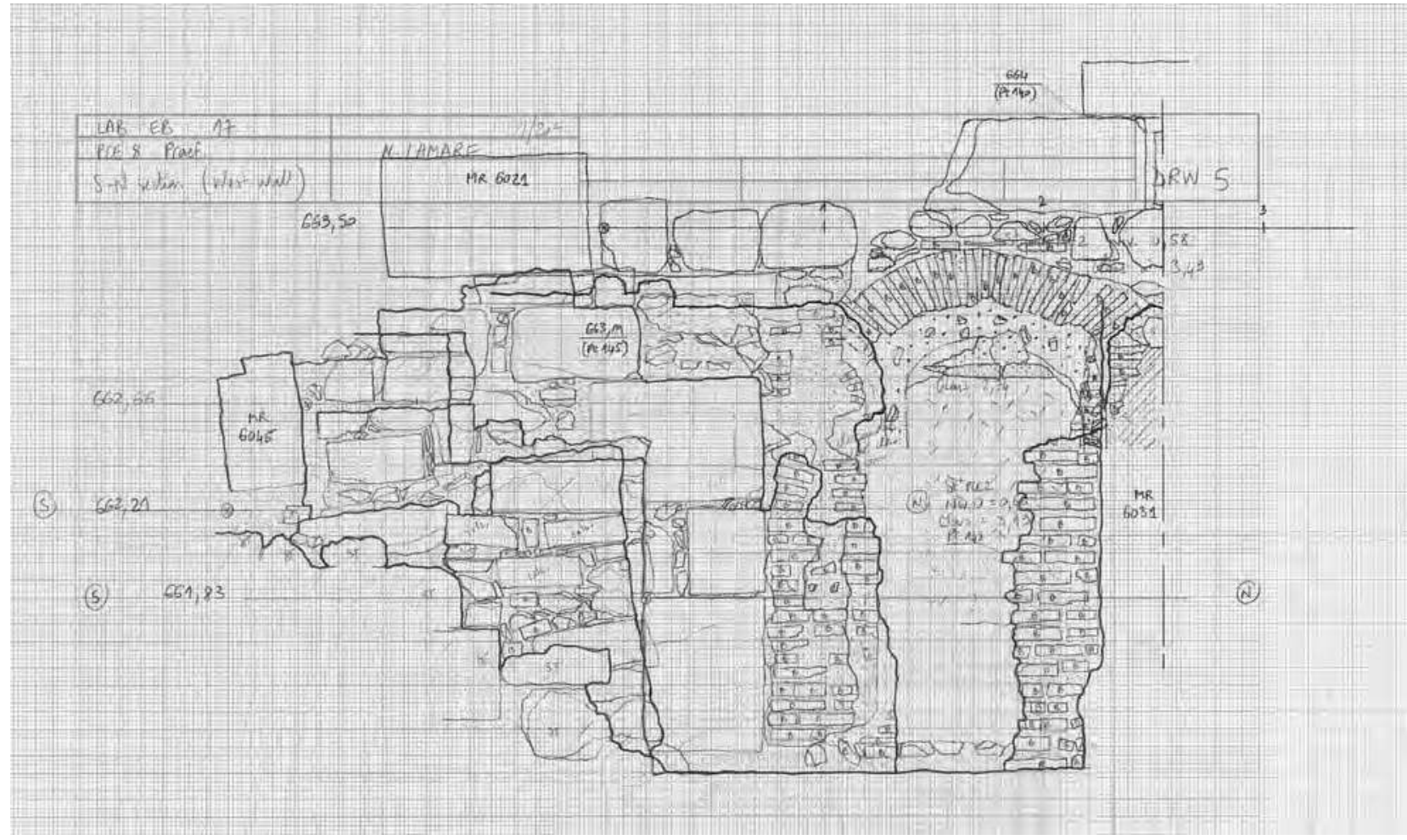

Fig. 6.2.18. : PCE8, praefurnium, élévation Sud-Nord, superposition des relevés (N. Lamare).

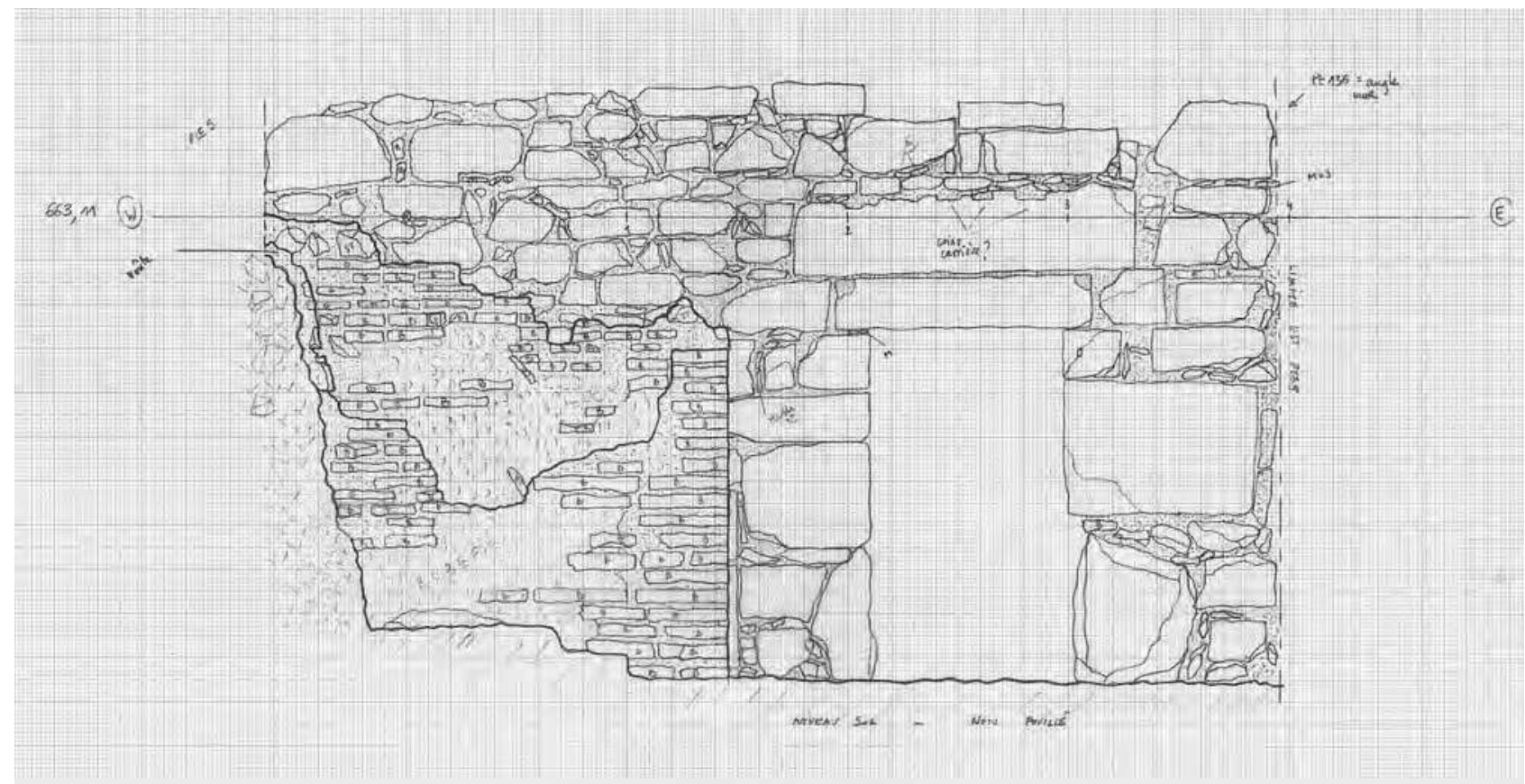

Fig. 6.2.19. : PCE8, praefurnium, élévation Ouest-Est (N. Lamare). 


\begin{tabular}{|c|c|c|c|c|c|c|c|}
\hline MR & Appareil & Largeur (m) & Module & Espèce & Liant & Helation & Réfection \\
\hline 6001 & Grand appareil carreaux et boutisses & 1,10 & & Gneiss & $\begin{array}{l}\text { Opus caementicium } \\
\text { foints secs }\end{array}$ & Lié 6003 & \\
\hline 6002 & $\begin{array}{l}\text { Grand appareil carreaux et bolutisses ; partie Sud } \\
\text { en moyen appareil avec pierres de calage } \\
\text { Double parement }\end{array}$ & $1,05-1,10$ & & Gnelss & $\begin{array}{l}\text { Opus coementicium } \\
\text { loints secs }\end{array}$ & & $\begin{array}{l}\text { Extension du mur vers } \\
\text { le sud (prolongement } \\
\text { trancon) }\end{array}$ \\
\hline 6003 & $\begin{array}{l}\text { Grand apparell carreaux et boutisses : } \\
\text { aménagement particulier pour orifice carre }\end{array}$ & 0,85 & & Gneiss & Opus caementicium & LiE 6001 & \\
\hline 6004 & $\begin{array}{l}\text { Grand appareil irrégulier } \\
\text { (pas de boutisses) avec plerres de calage }\end{array}$ & 1,40 & $\begin{array}{l}\text { Remploi blocs } \\
\text { origine DH dans } \\
\text { parement }\end{array}$ & Grieiss & $\begin{array}{l}\text { Mortler avec } \\
\text { gravats }\end{array}$ & & \\
\hline 6005 & $\begin{array}{l}\text { Grand appareil carreaux et boutisses } \\
\text { Double parement } \\
\text { [Pierre de calage : seulement au bord des portes, } \\
\text { remaniements] }\end{array}$ & 1,10 & $\begin{array}{l}1,10-1,45 \\
\text { assises } 0,55-0,65\end{array}$ & Gneiss & $\begin{array}{l}\text { Opus coementiciuen } \\
\text { Mortier dans foints } \\
\text { (résidu de } \\
\text { revêtement ?) }\end{array}$ & Lié 6002 & $\begin{array}{l}\text { Rétréci en partie ouest } \\
\text { (PCE1) }\end{array}$ \\
\hline 6006 & $\begin{array}{l}\text { Grand appareil irregulier avec pierres de calage } \\
\text { Double parement }\end{array}$ & & $\begin{array}{l}\text { Irrégulier (sans } \\
\text { doute remployés } \\
\text { sprès retailte) }\end{array}$ & Greiss & $\begin{array}{l}\text { Opus caementicium } \\
\text { loints mortler } \\
\text { blanc, calages } \\
\text { (éclats marbre) }\end{array}$ & $\begin{array}{l}\text { Lie 6002, } \\
6015\end{array}$ & \\
\hline 6007 & $\begin{array}{l}\text { Grand appareil réguller carreaux et boutisses } \\
\text { (type hellénistique) }\end{array}$ & 1,10 & & Gneiss & $\begin{array}{l}\text { Opus caementicium } \\
\text { Joints secs }\end{array}$ & $\begin{array}{l}\text { L.Lé 6018 } \\
\text { Posé contre } \\
\text { DH }\end{array}$ & $\begin{array}{l}\text { Reprise de l'élévation } \\
\text { pour le caldarium: } \\
\text { rétrécissement pour } \\
\text { aménagement } \\
\text { banquette (dans } \\
\text { épaisseur) }\end{array}$ \\
\hline 6012 & Grand appareil carreaux et boutisses & Incomplête & & & & avec 6007? & Arasé \\
\hline 6015 & $\begin{array}{l}\text { Grand apparell pseudo-isodome, avec pierres de } \\
\text { calage, traces attaches placage } \\
\text { Double parement carreaux et boutisses }\end{array}$ & 1.10 & $0,50-1,15$ & $\begin{array}{l}\text { Gneiss, } \\
\text { briques } \\
\text { calage }\end{array}$ & $\begin{array}{l}\text { Mortier blanc } \\
\text { entre les blocs } \\
\text { (pour calage) }\end{array}$ & $\begin{array}{l}\text { Lié 6002, } \\
6006\end{array}$ & \\
\hline 6016 & $\begin{array}{l}\text { Appareif mixte avec blocs et briques, assises } \\
\text { irrégulieres, parement simple }\end{array}$ & 0,50 & & Gneiss & Mortier blane & t.ee 6021 & $\begin{array}{l}\text { Cóté corridor, reprises } \\
\text { en briques }\end{array}$ \\
\hline 6017 & & & & & & $\begin{array}{l}=6003,46 \\
6028\end{array}$ & $\begin{array}{l}\text { Ouverture possible } \\
\text { pour conduite pédlluve }\end{array}$ \\
\hline 6018 & Grand appareil carreaux et boutisses d'origine & 1.50 & $1,35-2,00$ & Gneiss & Opus caementicium & $\begin{array}{l}=6022 \\
\text { Lié } 6007\end{array}$ & $\begin{array}{l}\text { Reprise : blargissement } \\
\text { pous installation } \\
\text { exédre, aménagement } \\
\text { contrefort }\end{array}$ \\
\hline 6021 & $\begin{array}{l}\text { Blocs et moyen appareili, avec assises de réglages } \\
\text { de briques }\end{array}$ & $0,50-0,55$ & $0,30-0,40$ long & $\begin{array}{l}\text { Gneiss et } \\
\text { briques } \\
\text { calage }\end{array}$ & Mortier & $\begin{array}{l}\text { Lié 6016, } \\
6023\end{array}$ & $\begin{array}{l}\text { Traverse par le canal de } \\
\text { chauffe }\end{array}$ \\
\hline 6022 & Grand appareil carreaux et boutisses d'origine & & $1,35-2,00$ & & $\begin{array}{l}\text { Joints secs } \\
\text { (comblement de } \\
\text { gravats vidé) }\end{array}$ & $=6018$ & \\
\hline 6023 & $\begin{array}{l}\text { Parement extérieur grand appareil carreaux } \\
\text { boutisses (hellénistique) } \\
\text { Double parement }\end{array}$ & 1,30 & & Gneiss & Opus coementicium & $\begin{array}{l}\text { démonté } \\
\text { poury } \\
\text { accrocher } \\
6021 \\
\text { (cf. angle) }\end{array}$ & $\begin{array}{l}\text { Reprise parement } \\
\text { intérieur } \\
\text { (aménagement bassin) }\end{array}$ \\
\hline 6024 & $\begin{array}{l}\text { Grand et moyen appareif carreaux et boutisses } \\
\text { Double parement }\end{array}$ & 0,80 & & Gneiss & Mortier & Lie 6004 & \\
\hline 6028 & Grand apparell à double parement & 1.15 & & & & $=6017$ & \\
\hline 6029 & $\begin{array}{l}\text { Mur de briques (double parement) avec bloc } \\
\text { niveau supérieur, piédroit gneiss }\end{array}$ & 0,38 & & & Mortier & $\begin{array}{l}\text { Contre 6017, } \\
6028\end{array}$ & \\
\hline 6030 & Double parement & 1,10 & $0,65-1,10$ & Gaeiss & $\begin{array}{l}\text { Opus ciementicium } \\
\text { Joints secs }\end{array}$ & & \\
\hline 6031 & Petit appareil irréguller & 0,60 & $\begin{array}{l}\text { Varlable; } \\
\text { montants porte } \\
\text { grand appareil }\end{array}$ & $\begin{array}{l}\text { Gneiss (et } \\
\text { autres ?). } \\
\text { briques. } \\
\text { marbre }\end{array}$ & Mortier & $\begin{array}{l}\text { Sappuie sur } \\
\text { 6021: } \\
\text { sappuie } \\
\text { contre } 6032\end{array}$ & \\
\hline 6032 & $\begin{array}{l}\text { Grands blocs de base, petite et moyen apparefl très } \\
\text { irrégulier (mur de soutênement?) } \\
\text { Double parement (non dégagê) }\end{array}$ & & $0,24 \cdot 0,50$ long & $\begin{array}{l}\text { Gineiss, } \\
\text { briques }\end{array}$ & Mortier & & \\
\hline 6045 & Un seul parement conservêt & & $\begin{array}{l}1,20 \text { (mesure } \\
\text { d'un bloc) }\end{array}$ & Gueiss & & $=6023$ & \\
\hline
\end{tabular}

Fig. 6.2.20. : Inventaire des murs des bains mis au jour (N. Lamare).

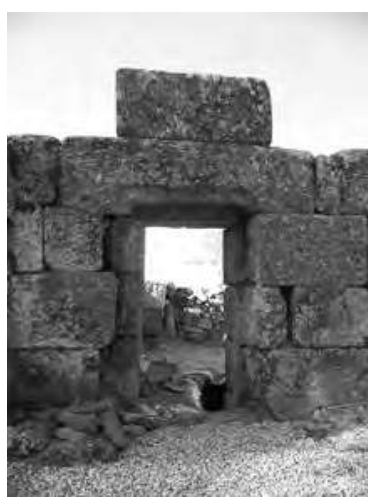

Fig. 6.2.21. : Eglise est, linteau taillé de la porte PT6008

(N. Lamare).

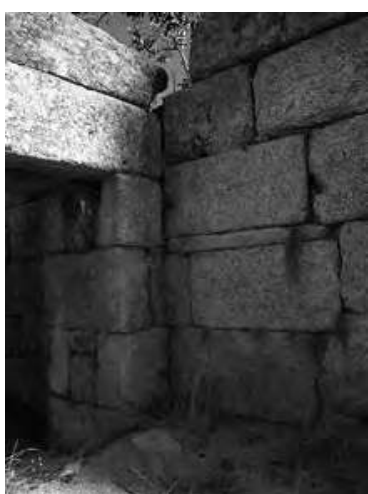

Fig. 6.2.22. : PCE2, angle des murs 6005 et 6002

(N. Lamare).

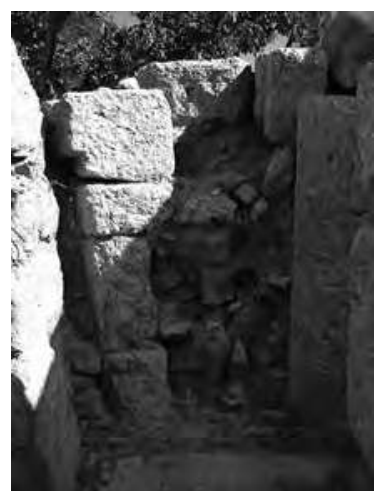

Fig. 6.2.23. : PCE7, porte (?) comblée dans le mur nord 6015

(N. Lamare) 
ractéristiques (6016 et 6021), de même que la cloison 6029.

- Petit ou moyen appareil irrégulier de moellons de gneiss associé à d'autres matériaux, en particulier des briques ou du marbre servant de calage, qui pourrait être caractéristique des aménagements les plus tardifs de la phase balnéaire (en particulier 6031 et 6032).

L'observation renouvelée du bâti permet d'identifier progressivement les remaniements successifs de l'ensemble de la construction. Au Nord, la porte 6008 présente une trace de taille importante sur son linteau du côté de l'église, pratiquée pour permettre un passage suffisamment haut pour une personne (Fig. 6.2.21). De plus, l'irrégularité des blocs aux angles avec les murs 6002 et 6003 indiquerait que ces derniers ont été ajoutés dans un second temps (Fig. 6.2.22). La porte 6009 est une ouverture aisément attribuable à une phase postérieure à la construction du mur en raison des grandes irrégularités du bâti. On peut ainsi supposer que le mur nord ne comportait ni cloisons si ouverture à ces endroits lors de son installation.

A l'extrémité du corridor PCE7 vers l'Ouest, un amas de gravats qui occupe l'épaisseur du mur a toujours rendu difficile la lecture de cette partie du bâti. Une observation mérite d'être mentionnée : à ce niveau du mur nord du corridor (6015), un bloc de grand appareil est placé en hauteur, de la même façon qu'à l'Est où il en marque l'extrémité (Fig. 6.2.23). Nous proposons d'identifier une porte dont le bloc formerait le montant : alignée avec l'extrémité du bassin de PCE5, elle ferait alors partie d'un aménagement concerté. Cependant, le parement nord du mur 6015 ne permet par d'identifier une ouverture. Il faut rappeler que cet angle dans lequel se croisent les murs 6002, 6003 et 6015 est l'un des plus complexes de l'édifice puisqu'il combine plusieurs remaniements. L'étude devra se poursuivre pour clarifier l'identification de cette partie du bâti, éventuellement en procédant à un nettoyage du comblement.

La poursuite de l'analyse des différents types de bâti permettra de préciser les phases de construction et les remaniements du secteur, au-delà de la phase balnéaire. Le travail actuel se concentre sur la mise au net des relevés de la dernière campagne et ceux des années précédentes. L'ensemble des structures mises au jour a été relevé en plan et en élévation, exception faite de la façade sud du mur 6015 : les observation faites sur cette dernière rendent donc son étude détaillée indispensable lors de la prochaine campagne.

\subsection{LA STOA NORD (par O. Henry)}

Parmi les nouveaux projets de la saison $2017 \mathrm{fi}-$ gurait la fouille de la stoa nord (Fig. 6.3.1 ${ }^{156}$. Cette dernière est localisée sur le terrasse du temple de Labraunda, installée au pied d'un haut mur de terrasse qui retient les terres de la pente en amont. La stoa est orientée parallèlement à ce mur de terrasse dont elle n'est séparée que de quelques dizaines de centimètres.

Cette stoa avait fait l'objet de recherches archéologiques en 1948 et 1953. Ces recherches, certes limitées, avait toutefois permis d'établir que le bâtiment, de fondation hékatomnide, avait été rebâtis dans le courant du $2^{\mathrm{e}} \mathrm{s}$. ap. J.-C. par un certain Poleites. De nombreuses questions toutefois restent en suspens, notamment concernant son plan et son orientation qui semblent inhabituels. La fouille de cet ensemble devrait aussi permettre d'éclairer la pratique du culte de Zeus Labraundos ainsi que le devenir de ce culte et de l'occupation de la terrasse du temple, plus généralement, aux périodes tardives.

\subsubsection{FOUILLES ANCIENNES ET ETAT DES LIEUX}

En 1948 et 1953, un total de trois sondages ont été pratiqués sur la Stoa nord, sous la direction de Karin Leman (en 1948) et de Alf et Gertrud Nordström (en 1953). En 1948, les opérations de fouille ont exclusivement concerné la partie orientale de la stoa. Un premier sondage (A) a été pratiqué au Nord de l'angle nord-ouest des propylées Y, dans l'espoir de mettre au jour le mur d'ante est de la stoa (Fig. 6.3.2). Au cours de ce sondage, un alignement de blocs, orientés Nord/Sud, fut interprété comme appartenant à ce mur d'ante, sa localisation ainsi que sa piètre conservation n'a cependant pas permis aux archéologues de confirmer cette identification. Nous pensons, à la lumière des éléments dont nous disposons aujourd'hui, qu'il s'agissait probablement de la face interne du parement ouest de ce mur d'ante. D'autre part, le sondage a aussi permis d'observer que l'ensemble de l'angle sud-est de la stoa semble avoir disparu, suite à un événement majeur. En lieu et place de cet angle, les archéologues mirent au jour un amas de grands blocs taillés qui reposaient dans les remblais sans aucune organisation précise. A la suite de l'opération, qui ne dura que quelques jours (les 28 et 29 juin 1948), ce sondage fut remblayé. Ce remblai est encore visible aujourd'hui, notamment le muret de pierres sèches

156) La fouille et les prises de note de terrain ont été réalisées par M. Hauchart, sous la direction de O. Henry. Elle était assistée de A. Arslan et C. Ardil. 


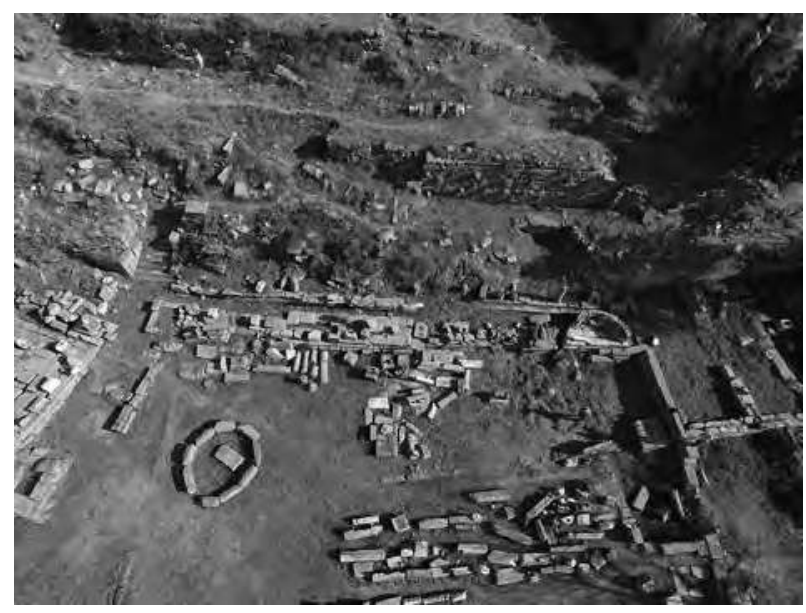

Fig. 6.3.1. : Vue aérienne de la stoa nord (O. Henry).

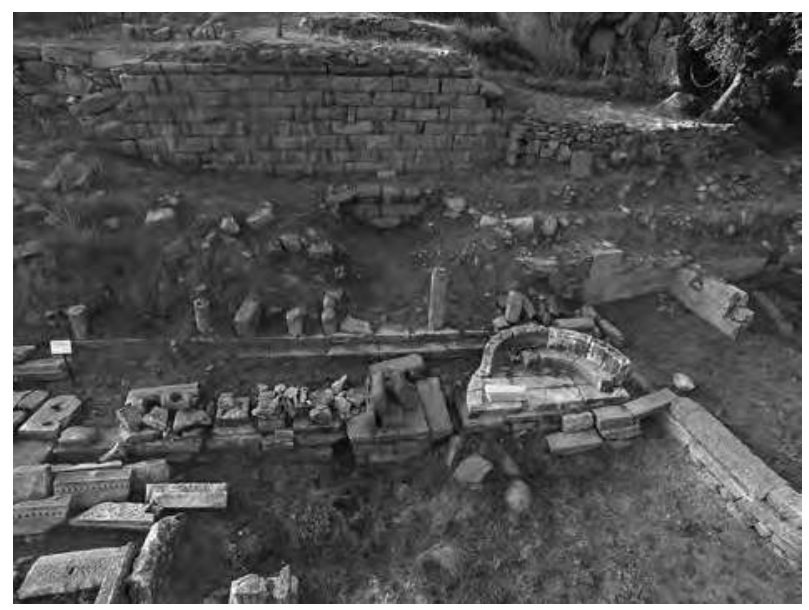

Fig. 6.3.3. : Extrémité orientale de la stoa nord avant la saison 2017 (O. Henry).

entre l'arrachage ouest du mur nord des propylées et un grand bloc posé de chant à l'Ouest (Fig. 6.3.3, au-dessus et à droite de l'exèdre).

A la suite de ce premier sondage, qui avait notamment permis de dégager le grand mur de terrasse au Nord, il fut décidé de pratiquer un second sondage (B), 4 m à l'Ouest du sondage A. L'objectif de ce sondage, très étroit, était de pratiquer une section complète Nord/Sud à travers la stoa afin de déterminer si ce grand mur de terrasse appartenait à la stoa ou non. Les travaux ont permis de dégager plusieurs nouveaux murs (Fig. 6.3.4). Le premier (B), "mal construit", fut construit parallèlement au stylobate, en arrière de ce dernier. Les archéologues l'identifièrent à un mur de terrasse relativement tardif. Le second $(\mathrm{F})$ s'avère être beaucoup plus imposant,

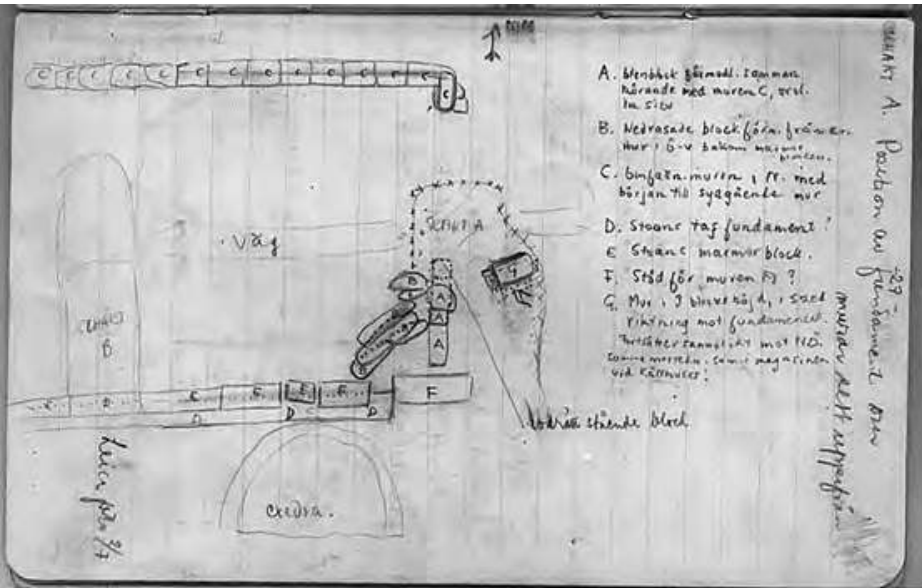

Fig. 6.3.2. : Extrait du journal de fouille de 1948, croquis du sondage $\mathbf{A}$ ( $\mathrm{K}$. Leman).

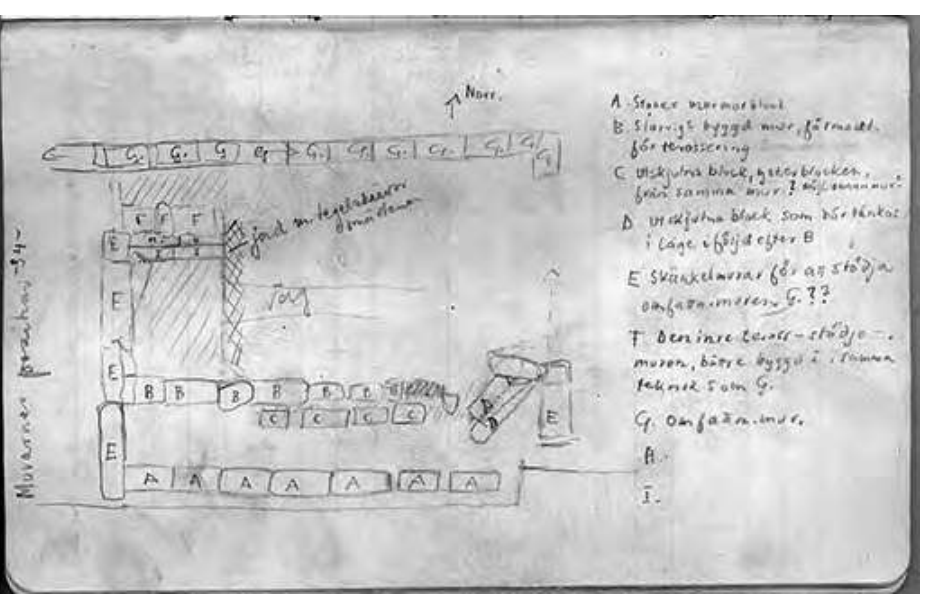

Fig. 6.3.4. : Extrait du journal de fouille de 1948, croquis du sondage B (A : stylobate ; B : mur secondaire ; $\mathrm{C}$ : blocs effondrés ; $\mathrm{D}$ : idem ;

$\mathrm{E}:$ mur transversal ; F : mur nord de la stoa ; G : mur de terrasse nord (K. Leman).

construit en carreaux et boutisses avec de larges blocs proprement taillés, parallèle au grand mur de terrasse $(\mathrm{G})$ et construit une quarantaine de centimètres en avant de ce dernier. Il s'agit du mur du fond de la stoa nord. Au pied de ce mur les archéologues observent une assise de blocs proprement arrangés, interprétés comme une probable banquette. Enfin, un troisième mur (E), orienté Nord/Sud, fut mis au jour à l'extrémité occidentale du sondage. Construit avec du matériel de remploi, utilisant souvent des blocs de grandes dimensions, il semble barrer intégralement la stoa puisqu'il s'appuie sur le mur du fond au Nord et se termine au niveau du stylobate au Sud. La fonction de ce mur, secondaire, n'a pas pu être définie et sa qualité même de mur est mise en doute. Il fut interprété comme un élé- 
ment de soutien pour le mur nord de la stoa (?). A la suite de ces opérations, qui se terminèrent le 3 juillet 1948, décision fut prise de remblayer l'ensemble du sondage.

Les fouilles menées sur la stoa en 1953 ont visé deux localisations. La première $(\mathrm{AH})$, à l'Ouest, avait pour but de mettre au jour l'extrémité occidentale de la stoa, au contact de l'escalier qui mène à la terrasse supérieure. Cette tranchée ne fut jamais terminée. Elle permit cependant de fixer avec certitude la localisation du mur d'ante occidental.

La seconde tranchée (AG), beaucoup plus importante, fut pratiquée une nouvelle fois dans la partie orientale de la stoa. Elle fut menée en deux temps : d'abord sur 2,50 m de large, puis étendue vers l'Ouest pour intégrer l'ancienne tranchée de 1948 et atteindre une largeur totale de $5 \mathrm{~m}$ (Fig. 6.3.5). Les informations récoltées au cours de cette campagne, outre qu'elle ont permis de mieux voir le mur nord de la stoa, n'ont pas apporté d'éléments absolument nouveaux pour la compréhension du bâtiment.

Ces deux saisons de fouilles, accompagnées par une étude des blocs d'architecture situés sur la terrasse du temple, menée en 1983, 1993 et 1994, résulta dans la publication d'un long article en 1996 dans le journal Opuscula Atheniensia ${ }^{157}$. Signé par P. Liljenstolpe et P. von Schmalensee, cet article est essentiellement dédié à la reconstruction historique et architecturale de la stoa nord. Cet article révèle que la stoa fut d'abord probablement construite par Maussollos lui-même avant d'être remaniée et/ ou reconstruite au deuxième siècle ap. J.-C. par un certain Poleites. D'après les informations contenues dans la (longue) dédicace architravale, celle-ci semble avoir été gravée entre 102 et 114 ap. J.-C. Dans son état romain, la stoa mesurait entre 27,2 et $29 \mathrm{~m}$ de long. Elle comportait une façade de 12 colonnes in antis, d'ordre corinthien.

La reconstruction architecturale des auteurs, si elle ne pose pas de problème quand à la section du bâtiment, est beaucoup plus sujette à caution concernant le plan proposé (Fig. 6.3.6). En effet, ce dernier révèle un écart extrêmement important de la profondeur de la stoa à ses extrémités est et ouest, provoqué par une différence d'orientation entre le stylobate et le mur du fond du bâtiment. L'absence de certitude concernant l'exacte position du mur d'ante oriental soulève de nombreuses questions, notamment quant à l'étendue du bâtiment, et au nombre de colonnes. D'autres questions se posent également quant à l'étendue de la reconstruction ro- maine de la stoa hékatomnide, à sa raison d'être. On ignore aussi complètement le déroulé de l'histoire de la stoa, dans le détail, puisqu'aucun élément issu des travaux de fouille et de la stratigraphie mise au jour n'est mentionné ici.

\subsubsection{FOUILLES 2017}

L'objectif des fouilles entreprises sur la stoa nord cette année est double. Il s'agit, d'abord, de mener une fouille en stratigraphie, afin de connaître davantage de détails quant à l'histoire de la construction et de l'occupation du bâtiment. Situé sur la terrasse du temple, il ne fait aucun doute qu'il comporte de précieuses informations, notamment sur les périodes tardives du site et le devenir du culte de Zeus Labraundos à l'époque paléochrétienne. La fouille pourrait également offrir des informations sur la fonction de cette stoa dans le déroulement du culte de Zeus.

Ensuite, cette opération est également motivée par des raisons de restauration, de protection et de mise en valeur du site de Labraunda. La stoa nord représente un des bâtiments majeurs de la terrasse du temple, bien mieux conservée, semble-t-il que ce dernier. Une fouille intégrale du bâtiment permettrait de dégager un ensemble spectaculaire, au cœur du site. Suite à la fouille nous avons notamment l'intention de mettre en valeur les éléments architecturaux du bâtiment, notamment la suite de 10 blocs d'architraves (dont 6 inscrits) portant des moulures extrêmement bien conservées.

Enfin, il est évident, pour quiconque visite le site de Labraunda, que le mur de terrasse nord, dont la partie occidentale a été arraché, montre un contre-fuit très dangereux. Afin de mettre en place un projet de consolidation de ce mur il est impératif d'en connaître l'ensemble des caractéristiques. La stoa nord, et notamment l'important remblai qui la couvre, nous empêche à l'heure actuel d'observer les parties inférieures du mur de terrasse. La fouille de la stoa nous permettra donc de compléter la documentation du mur de terrasse nord et de procéder, dans les conditions les meilleures, à sa consolidation.

Le but du sondage effectué cette année dans la stoa nord était de redégager l'ancienne tranchée AG, creusée en 1953 et d'établir une première analyse stratigraphique du bâtiment. Bien que presqu'intégralement remblayée par le colluvionnement, la localisation de l'ancienne tranchée était encore visible grâce à une dépression dans le sol, d'où émergeait l'arase du mur nord de la stoa (Fig. 6.3.3). 


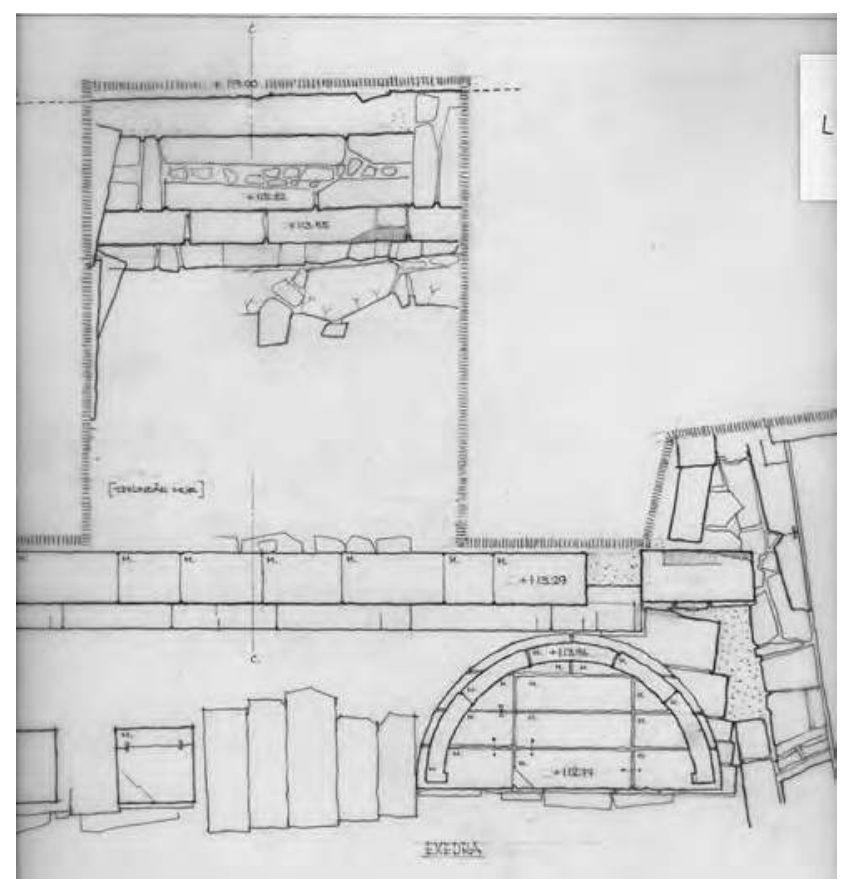

Fig. 6.3.5. : Extrait du journal de fouille de 1953, relevé en plan du sondage AG (G. Nordström).

L'opération a commencé par un sérieux nettoyage de la zone. Ensuite, nous avons creusé au centre de la dépression ancienne pour retrouver les niveaux atteints par l'équipe suédoise. Nous avons donc ainsi mis au jour l'intégralité de l'élévation conservée du mur nord, sur trois assises, ainsi que la banquette mentionnée dans les carnets de fouille. Les remblais dégagés, il semblait clair que nous n'avions toujours pas atteint les limites du sondage ancien et nous avons décidé d'élargir le sondages vers l'Ouest dans un premier temps. Cette extension a rapidement buté sur un amas de très larges blocs, empilés les uns sur les autres, ce qui correspond au mur E mentionné plus haut. Nous avons décidé de stopper l'extension du sondage à cet endroit. Afin de prévenir tout effondrement future de ces blocs, nous avons bâti un mur de pierre, parallèle au "mur" $\mathrm{E}$, à partir des blocs contenus dans les remblais que nous avons dégagés (Fig. 6.3.7).

Ces derniers, d'une hauteur dépassant parfois $2 \mathrm{~m}$ au-dessus du niveau du stylobate étaient composé de terre colluvionée depuis les côtés de l'ancien sondage ou de la pente située en amont du mur de terrasse nord pour la partie supérieure, puis d'un mélange très dense de terre meuble et de large blocs de gneiss quadrangulaires. Ce dernier niveau, qui semble continuer bien en dessous du niveau du stylobate correspond sans aucun doute à

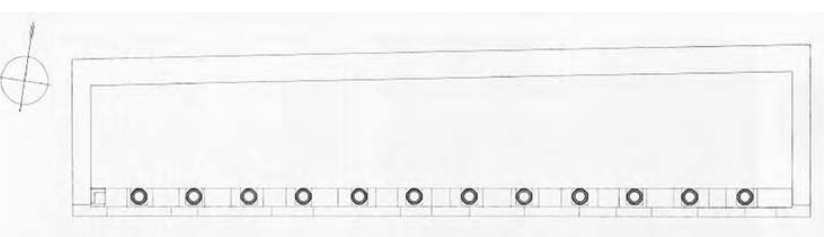

Fig. 6.3.6. : Plan restitué de la stoa nord

(Liljenstolpe et Schmalensee 1996 : fig. 32)

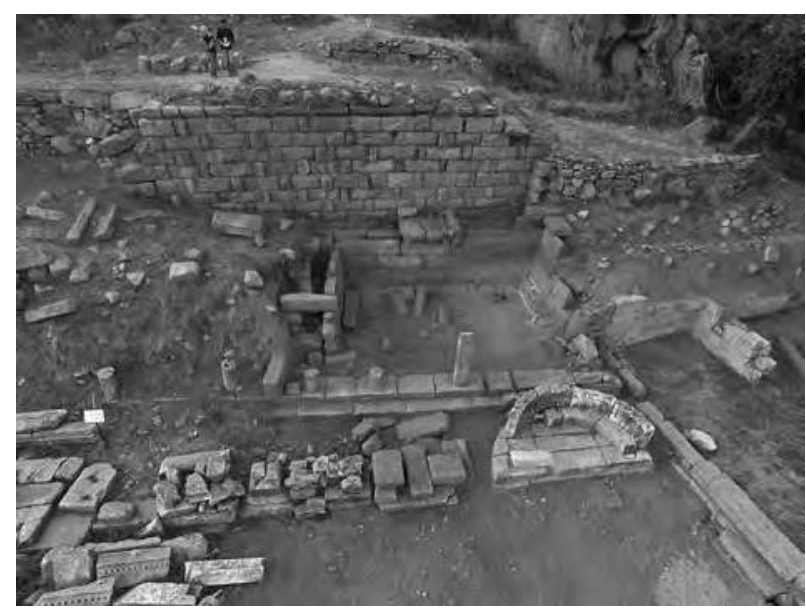

Fig. 6.3.7. : Extrémité orientale de la stoa nord après la saison 2017 ( $O$. Henry).

un remblai volontaire, probablement pratiqué par les archéologues en 1953, à moins qu'il ne s'agisse de l'œuvre des paysans locaux. Ces derniers utilisaient en effet, jusqu'à récemment, la stoa nord comme chemin de traverse au pied du grand mur de terrasse nord. Nous avons volontairement stoppé la fouille au niveau du stylobate de la stoa, nous réservant le droit d'effectuer une tranchée profonde ultérieurement. Le nettoyage du sol à cet endroit a par ailleurs démontré la présence vers l'Ouest, d'une très grande fosse, remplie de pierres non taillées de petit module. Cette fosse semble se poursuivre sous le mur E. Il se pourrait qu'elle soit antérieure aux recherches menées en 1948 et en1953 et appartienne à un état d'occupation dont nous ignorons la date. Les sondages pratiqués à ces époques s'étaient en effet arrêtés après avoir buté contre le mur E.

Notre sondage a ensuite été étendu vers l'Est, dans l'espoir de mettre au jour l'angle du bâtiment et donc le mur d'ante oriental de la stoa. Vers l'Est la stratigraphie était beaucoup plus complexe, même si sa majeure partie est composée de vagues successives de colluvionnement. Il semble que la dernière unité stratigraphique rencontrée (US2016) corresponde à la phase d'abandonnement/destruction (?) puisqu'elle montre un substrat composé de limon brun noir homogène et meuble, avec des inclusions 


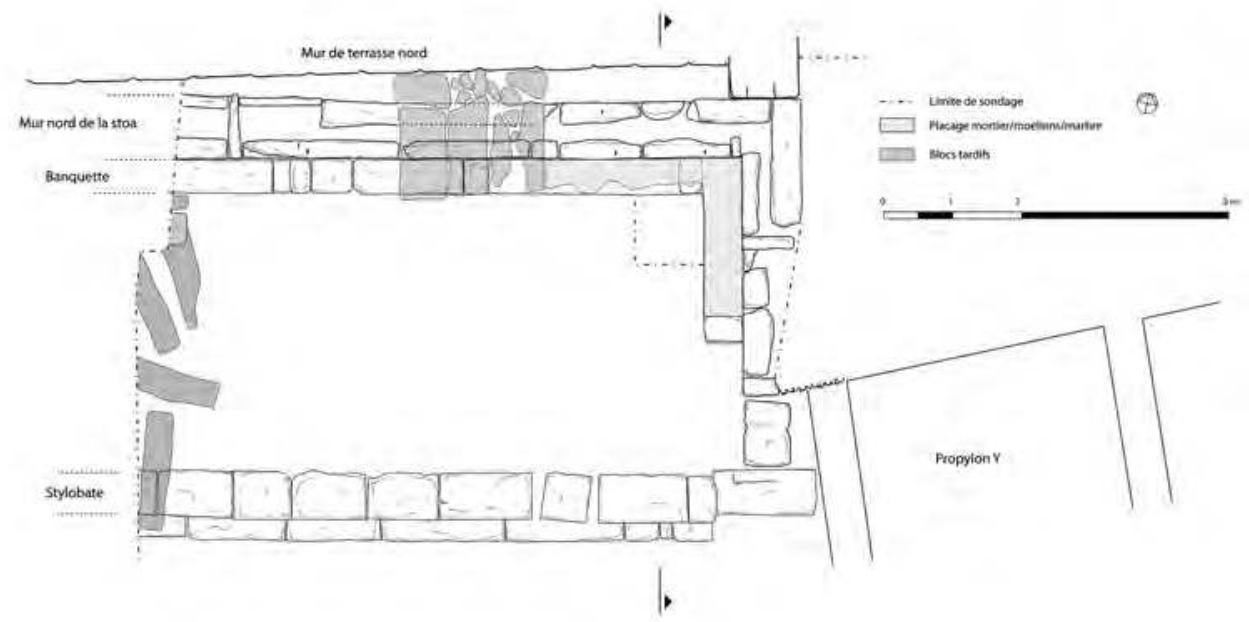

Fig. 6.3.8. : Relevé en plan des vestiges mis au jour (M. Hauchart et O. Henry).

de nodules de TCA, de céramiques et des fragments de bronze, des cailloux, du gravier et du charbon.

L'extension du sondage vers l'Est a aussi permis de révéler que la banquette située au pied du mur nord est continue jusqu'à l'angle, puis qu'elle longe le mur d'ante sur une distance de $2,75 \mathrm{~m}$ (Fig. 6.3.8-9). Cette banquette est formée de longs blocs de gneiss, accolés aux murs nord et est. Bien qu'elle soit parfaitement horizontale, sa face supérieure comporte une série de "coussins" proprement taillés, d'une dizaine de centimètres de haut, larges de 30 à $35 \mathrm{~cm}$ et régulièrement espacés. Mais encore, des vestiges de mortier hydraulique ainsi que de nombreux placages en marbre conservés in situ, nous indiquent que l'angle intérieur de la stoa était décoré d'un placage de marbre qui recouvrait non seulement la banquette (qu'elle rehausse de $20 \mathrm{~cm}$ ) ainsi que sa face intérieure, mais aussi le parement interne des murs de la stoa (Fig. 6.3.10). Une série de petites mortaises circulaires (qui pourraient tout à fait correspondre à des fixation "hautes" d'un placage de marbre), placées à la même hauteur (environ $120 \mathrm{~cm}$ au-dessus du stylobate) semble marquer la limite supérieure de ce placage sur les murs. Ces mortaises sont limitées à l'angle de la stoa; sur le mur nord la dernière mortaise est situé à $1,80 \mathrm{~m}$ de l'angle, sur le mur d'ante il est difficile de se prononcer en raison du mauvais état de conservation, mais il est peu probable que le placage ait dépassé la banquette elle-même (soit une longueur maximum de $2,75 \mathrm{~m}$ ).

Enfin, nous avons pratiqué un modeste sondage dans l'angle nord-est, au pied de la banquette. Ce creusement nous a permis de confirmer que le placage de marbre se poursuit sur la face avant des blocs de la banquette, mais sans toutefois en at- teindre l'extrémité inférieure. Un arrachement du mortier, conservé à l'horizontal semble marquer la présence d'un niveau de sol, malheureusement disparu. Cet arrachement se trouve à une altitude identique à celle des blocs du stylobate, ce qui confirmerait qu'il s'agit bien là de l'altitude correspondant au niveau de sol.

La céramique récoltée au cours de cette opération est abondante et fera l'objet d'une étude spécifique dans le courant de la saison 2018.

A la suite de la fouille, nous avons procédé à une documentation complète du secteur comprenant couverture photographique mais aussi relevés de précision de plan et sections au $1 / 10^{\mathrm{e}}$, au $1 / 20^{\mathrm{e}}$ et au $1 / 50^{e}$. Le plan du secteur ainsi révélé nous apprend que :

- l'angle très aiguë formé par le mur d'ante de la stoa nord et le mur de façade ouest des propylées $\mathrm{Y}$ explique probablement la faiblesse structurelle repérée dans cette zone, ce qui est probablement à l'origine de la destruction partielle de la moitié sud du mur d'ante, et de la disparition du parement externe du mur d'ante ;

- le mur nord de la stoa et le mur de terrasse ne sont pas parallèles et forment un angle, mais qui semble moins marqué que ce qui est indiqué dans la publication de 1996, ce qui laisse envisager un plan général légèrement différent de celui présenté dans ladite publication ;

- le retour oriental du mur nord de terrasse forme un angle parfaitement aligné avec le mur d'ante de la stoa. Les deux murs ne semblent pas chaînés et le retour du mur de terrasse nord semble directement s'appuyer sur l'angle nord-est de la stoa ;

- l'essentiel de l'élévation des murs de la stoa offre un très beau parement. Le mur est construit à 


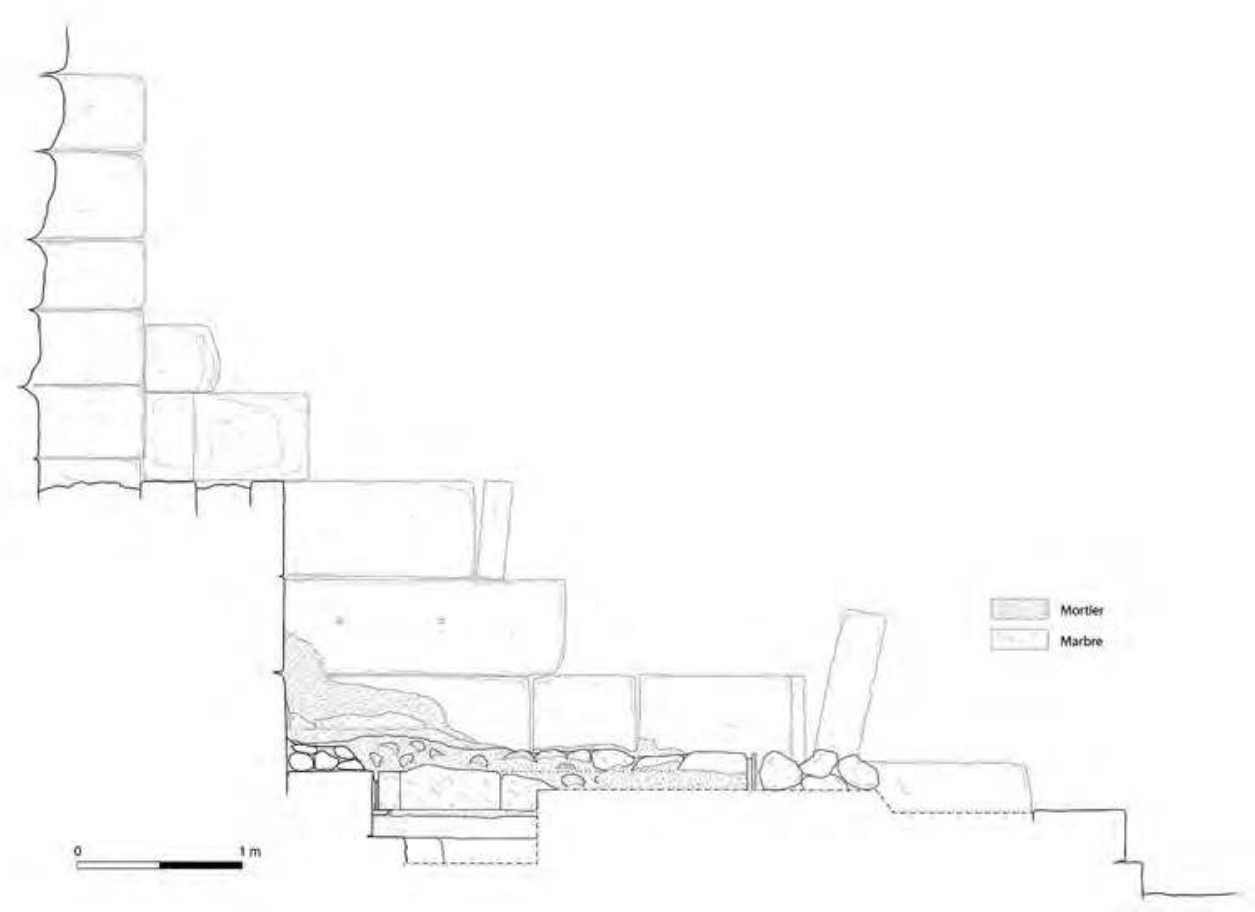

Fig. 6.3.9. : Section Nord/Sud des vestiges mis au jour (C. Ardil et O. Henry).

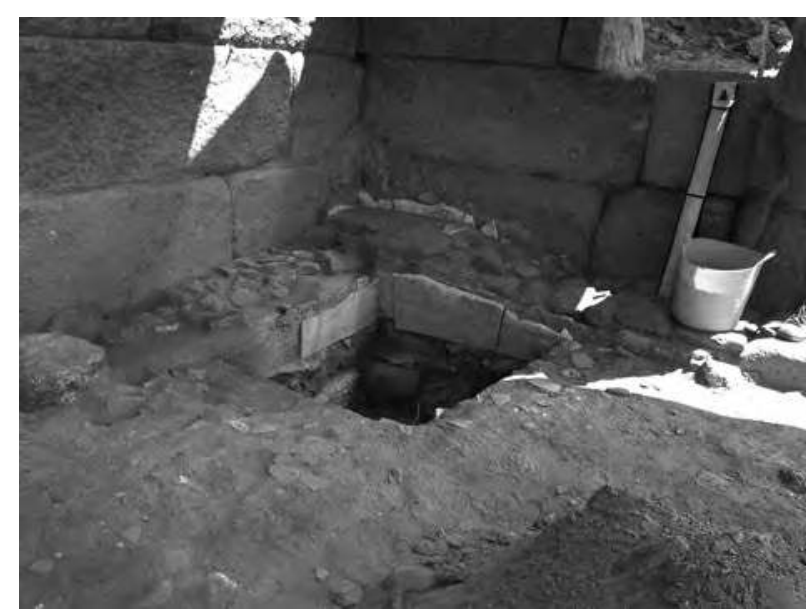

Fig. 6.3.10. : La banquette habillée de marbre et sondage profond dans l'angle nord-est de la stoa nord (M. Hauchart).

double parement à carreau et boutisse (on remarquera une absence de boutisse dans la rangée d'orthostate) et remplissage intérieur. Cette technique de construction semble davantage correspondre à une architecture hékatomnide plutôt que romaine. Des fragments de céramique à vernis noir mis au jour dans le remplissage des murs pourraient confirmer cette impression. Il semblerait donc que la reconstruction romaine n'ait pas touché à ces éléments du bâti ;

- on s'interrogera sur la présence de la banquette et de ses "coussins" taillés et régulièrement espacés. Leur fonction nous échappe pour l'instant, à moins d'imaginer que lesdits coussins aient pu servir de support à un élément en élévation (pilier, colonne) qui ne déparerait pas dans un décor d'époque romaine. Si tel est le cas, il est fort possible que cette banquette soit à attribuer à l'époque romaine. Dans ce cas-là, le placage de marbre qui couvrait l'angle du bâtiment jusqu'à une hauteur de 1,20 m appartiendrait à une phase de remaniement ultérieur.

\subsubsection{PROGRAMME FUTURE D'INTERVENTION}

Compte tenu du fait que la consolidation du mur nord de terrasse, qui présente un sérieux contrefruit, ne peut être envisagé sans que l'intégralité de son parement soit mis au jour, il est impératif que la fouille se poursuive vers l'Ouest. Dans le même temps, il est évident que la mise au jour des parties inférieures du mur accroît la sensibilité de ce dernier à la poussée des terres en amont.

Dans ces conditions, nous prévoyons de mener, de concert, la consolidation du mur et sa fouille. Ainsi, le principe est de consolider en 2018 la partie mise au jour en 2017, avant de reprendre la fouille. De cette manière, nous travaillerons par section, d'Est en Ouest. En fonction des financements disponible dans les années à venir, il sera envisagé d'effectuer sur une même saison la consolidation de la section fouillée l'année précédente et d'ouvrir une nouvelle section, soit de procéder à ladite 
consolidation, en reportant la poursuite de la fouille à la saison suivante. A condition de pouvoir mener de concert fouille et consolidation, nous prévoyons que l'étude archéologique de la stoa pourra être complétée en trois saisons, de 2018 à 2020. Si les financements nous imposent d'alterner saison de consolidation et saison de fouille, alors il faudra alors compter sept saisons complètes, soit de 2018 à 2024.

Une fois la stoa intégralement dégagée, nous prévoyons de mettre en valeur ce bâtiment, à l'aide notamment de panneaux de signalisation, ainsi qu'à travers la présentation, in situ, des 10 blocs d'architrave (dont 6 portent une dédicace) conservés, à l'image de ce qui avait été fait pour les architraves des Oikoi.

\subsection{WATER POOL (par Ö. Çakmaklı)}

The aim of the 2017 season was to understand the structure in its totality as well as the technology behind its function in the general water management at Labraunda.

In 2015, we removed the layers context 1 and 2 completely from the lower terrace structure, also known as trench 2 . In order to define better the stratigraphy of the building, we made a sondage trench $3.84 \times 2.17 \mathrm{~m}$ to the east of the structure in the north/south direction. The same trench has been extended towards the west in 2017 (Fig. 6.4.1).

This trench has a dimension of 3.80 by $3.10 \mathrm{~m}$. It has been divided into 4 sections, and the work started in the northern part G1 and G2 at the level of $655.42 \mathrm{~m}$ asl. During this work, two water pipes were discovered in the northern wall, almost at the same level, one being at $655.68 \mathrm{~m}$ and the other one at $655.67 \mathrm{~m}$ (Fig. 6.4.2). The first water pipe was found closed with a fragment of a tile, therefore it is understood that when this structure was used as a pool, this water pipe was probably out of use.

After G1 and G2, we continued working in the southern section (G3 and G4). All the excavations in 2017 ended at the level of the floor of the basin, composed of bricks arranged in herringbone (at $654.12 \mathrm{~m}$ asl). This brick revetment has been protected at the end of the season. At the same time, adjacent to northern and southern walls, an opening in each section (G1 to G4), in the form of a small sounding of 25 to $50 \mathrm{~cm}$ deep, has been opened in order to control whether the floor was preserved or not. It is understood that the floor is continuous at a level of $654.03 \mathrm{~m}$ asl. The finds from this season are similar to those from the earlier trench 2 and include ceramics (cooking, daily use vessels and thin walls bowl), loom weight of various sizes, glass (fragments of calice and bottles), metal and bones. In addition, in section G4, at a level of $655.16 \mathrm{~m}$ asl, and in section G1 at a level of $655.96 \mathrm{~m}$ asl, two bronze coins that are probably dating into the Hellenistic times have been found. Among the numerous finds, one has to emphasize the presence of water pipes and fragmentary bricks, which is not surprising considering the function of the building. Among all the excavated sections, the context 7 , immediatly above the floor, continue to be the most intact and best protected anthropic layer. Ceramic bowls and candle fragments (Fig. 6.4.3) dating to the $1^{\text {st }} \mathrm{c}$. AD have been found in high amount in this context.

The excavation of the water pool will continue in the future seasons.

\section{CONCLUSION (par O. Henry)}

La (double) mission 2017 a été particulièrement prospère, non seulement du fait que nous ayons débuté un programme de prospection parallèlement à la fouille elle-même, mais aussi car de nouveaux projets ont vu le jour. Ainsi les travaux qui ont débutés sur la stoa nord apportent déjà de nombreux éléments de réflexions quant aux diverses réaménagements romains qui intervinrent dans le courant du $2^{\text {e }}$ s. ap. J.-C. Ils permettent aussi de mieux connaître un des rares bâtiments encore peu étudié de la période hékatomnide. La réflexion est tout aussi valide pour l'étude de la stoa est qui a commencé en 2017. Il s'agit probablement du plus grand bâtiment construit par les hékatomnides (tout au moins en superficie). Sa fouille devrait débuter dans le courant de la saison 2018. Concer- nant l'étude du matériel de fouille, l'analyse du matériel métallique, entamée cette année, a déjà permis de démontrer la grande variété des formes et des alliages que le site recèle, et notamment de nombreux éléments qui permettraient d'envisager l'existence d'un atelier à Labraunda même. Cette étude, importante pour la compréhension de l'histoire du site, est aussi vitale pour la conservation du matériel. Certaines pièces qui avaient été conditionnées dans les années 1960 et 1970 ont déjà grandement souffert. Nous comptons donc mettre en place une approche globale de ce matériel qui inclura notamment la conservation de ces pièces parfois exceptionnelles.

A l'inverse, quelques projets qui étaient prévus pour la saison 2017 n'ont pas pu être réalisés, pour 


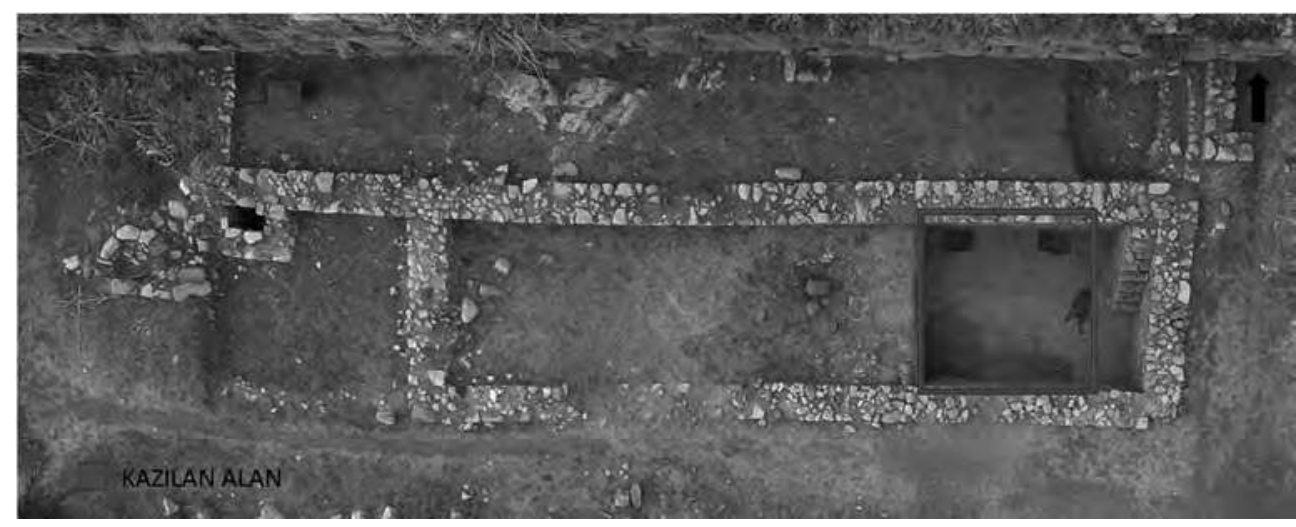

Fig. 6.4.1. : Aerial view of the roman pool, with the area excavated in 2017 (O. Henry).

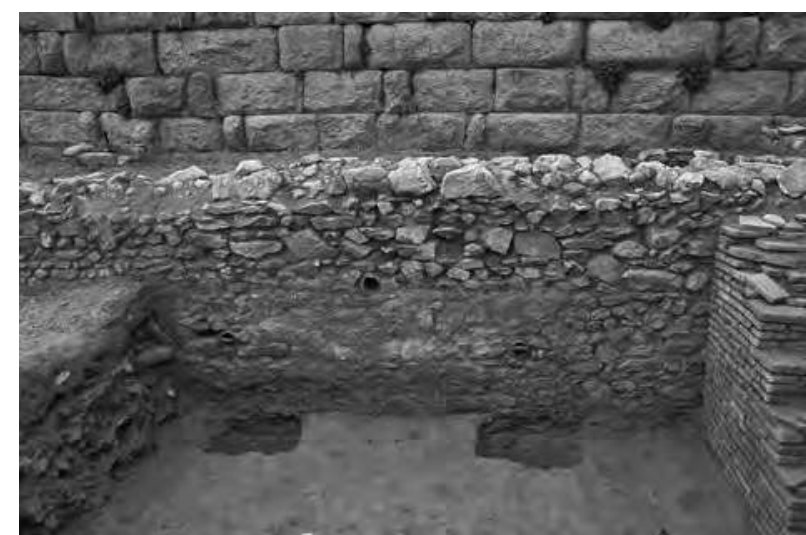

Fig. 6.4.2. : Southern face of the north wall of the roman pool (Ö. Çakmaklı).

des questions budgétaires autant qu'administratives. Il en est ainsi pour l'étude du stade qui aurait dû débuter cette année, sous la responsabilité d'un collègue roumain ; c'est aussi le cas de la fontaine hypostyle, débutée il y a trois ans, et qui n'a pas pu reprendre cette année. Nous espérons vivement pouvoir corriger ces contretemps dès l'année prochaine.

A la suite de l'Andrôn A, des Oikoi, de l'église Est et de la maison dorique cette année, nous poursuivons aussi la protection ainsi que la mise en valeur des différents bâtiments du site. Nous comptons déposer, dans le courant de l'hiver 2017/2018, une nouvelle candidature au prix Del Duca décernée par l'académie des inscriptions et belles lettres. Un tel financement permettrait de mettre en place un projet ambitieux et global, qui prendrait le pas sur les actions que nous menons de manière encore trop ponctuelle.

Enfin, nous tenons à remercier ici, encore une fois, l'ensemble des partenaires et sponsors de la mission de Labraunda, le MEAE en premier lieu et l'entreprise Axa, sans lesquels la mission 2017 n'aurait pas existé.

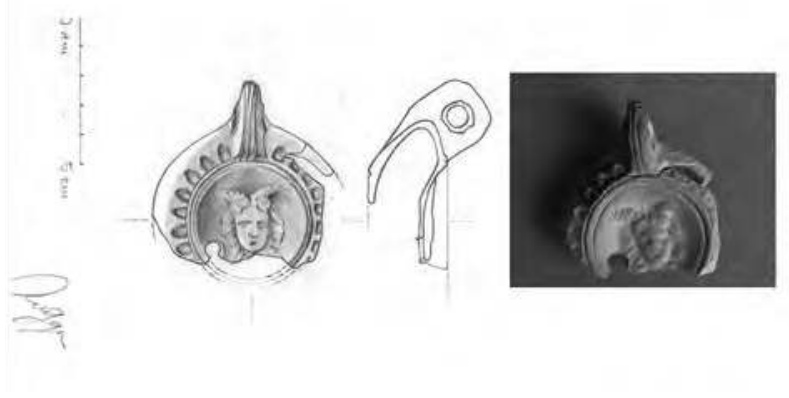

Fig. 6.4.3. : A roman lamp from the 2017 excavation in the roman pool (Ö. Çakmaklı).

La saison 2018 comportera, comme en 2017, deux volets, l'un consacré à la prospection étendue autour du site de Labraunda, l'autre sur le site lui-même.

La mission de prospection, qui durera trois semaines, aura pour objectif de : finir la couverture de la zone ouest, telle que définie dans le projet, et notamment de compléter l'ensemble des relevés des peintures rupestres de Sarıkaya ; d'entamer la prospection dans le quadrant oriental où de nombreuses structures ont déjà été repérées par nous-même au cours de visites ponctuelles.

La mission sur le site, qui durera deux mois, aura les objectifs suivants :

- Conservation/restauration : nettoyage et protection des marbres d'architecture ; protection et restauration du sol (quart sud-ouest) de la maison dorique; protection de l'arase des murs des Oikoi ; mise en valeur et protection du bâtiment hypostyle ; restauration des céramiques ; protection/ restauration des objets métalliques ; consolidation du grand mur de terrasse nord.

- Documentation : couverture photogrammétrique du bâti restant ; nettoyage et relevé de di- 
verses fontaines aménagées dans le territoire de Labraunda ; nettoyage et relevé des pièces sud de la stoa est ; compléter l'analyse de la TCA ; entamer l'analyse des placages de marbre issus des Bains Est ; nettoyage et documentation du stade.

- Fouilles : poursuite de la fouille de la stoa nord ; débuter la fouille de la stoa est ; débuter la fouille du secteur ouest (autour des bains tétraconques) ; terminer la fouille du bassin du bâtiment hypostyle ; poursuivre la fouille du bassin romain ; poursuivre et terminer la fouille de la "presse Persson".

O. Henry et al.

\section{BIBLIOGRAPHIE}

Agora V = Robinson, H.S. 1959 : Pottery of Roman Period Chronology, The Athenian Agora V, Princeton.

Agora XII = Sparkes, B.A. et Talcott, L., 1970 : Black and Plain Pottery of the $6^{\text {th }}, 5^{\text {th }}$ and $4^{\text {th }}$ Centuries B.C., The Athenian Agora XII, Princeton, NJ.

Agora XXXII = Hayes, J. $2008:$ Roman Pottery: Fine-Wares Imports, The Athenian Agora XXXII, Princeton, New Jersey.

Agora XXXIII = Rotroff S. $2006:$ Hellenistic Pottery, The Plain Wares, The Athenian Agora XXXIII, Princeton.

Alcock, S.E., 1993 : Craeca Capta: the landscapes of Roman Greece, Cambridge.

Atlante : AAVV, Enciclopedia dell'arte antica classica e orientale : Atlante delle forme ceramiche I, II, Istituto della Enciclopedia Italiana, Rome 1981, 1985/6. Tipologia (Hayes) : vol. II, 1-96 e 2 pp. aggiunte.

Baran, A., 2011 : "The Sacred Way and the spring houses of Labraunda sanctuary", in L. Karlsson et S. Carlsson (éds.), Labraunda and Karia (Boreas 32), Uppsala : 51-98.

Bingöl, O., 2014 : "Der arkitekturstil und seine Vorläufer", in Antike Malerei zwischen Lokalstil und Zeitstil, Wien : 239-244.

Blid, J., 2016 : Remains of Late Antiquity. Labraunda 4. Stockholm.

- 2012: Felicium Temporum Reparatio. Labraunda in Late Antiquity (c. AD 300-600), Stockholm.

Bonifay, N., 1986 : "Observations sur les amphores tardives à Marseille d'après les fouilles de la Bourse (1980-1984)", Revue Archéologique de Narbonnaise 19 : 269-305.

Çakmaklı, Ö.D., 2017 : "Preliminary results from the ceramics at the Water Pool excavations", in O. Henry et al. "Labraunda 2016 ", Anatolia Antiqua XXV : 227-229.

Caradini, A. et al., 1970 : "Ostia: le terme del Nuatatore I-IV"= Studi Miscellanei 13, 16, 21, 23, Rome 1968/9, 1970, 1973, 1977.

Coutelas, A., 2015 : "Etude pétroarchéologique de quelques revêtements muraux de l'Andrôn A de Labraunda (Turquie), non publié.

Crampa, J., 1972: The Greek inscriptions, part 2: 13-133 (ActaAth-4 ${ }^{\circ}, 5: 3: 2$ ), Labraunda III:2, Stockholm.

Dahlén, I., 1951 : Dagbok för område $P=$ doriska byggnaden, öster om K-propyléerna, sommaren 1951, (unpublished excavation diary).
Doğer, L., 2007 : "Byzantine ceramics: Excavations at Smyrna Agora (1997-98 and 2002-03)", in B. Bohlendorf, A.O. Uysal et J. Witte-Orr (éds.), Late Antique and medieval pottery and tiles in Mediterranean archaeological contexts (Byzas, 7), Istanbul : 97-122.

Doksanalt1, E., 2010 : "The Coarse Ware from a 'Late Roman House' in Knidos", in S. Menchelli, S. Santoro, M. Paquinucci et G. Guiducci (éds.), LTCW 3. Late Roman Coarse Wares. Cooking Wares and Amphorae in Mediterranean, Archaeology and archaeometry. Comparison between western and eastern Mediterranean, Vol. II BAR IS 2185 (II), Oxford : 769-780.

Empereur, J-Y et Picon, M., 1989 : "Les régions de production d'amphores impériales en Méditerranée orientale", in Amphores romaines et histoire économique. Dix ans de recherches, Acte du colloque de Sienne (22-24 mai), Rome : 223-248.

Eretria XXI : S. Fachard, Eretria XXI, La défence du territoire, Etude de la chôra Erétrienne et de ses fortifications, Gollion.

Ergenç, D., 2015 : "Mortiers hellénistiques et romains", in O. Henry et al., "Labraunda 2014", Anatolia Antiqua XXIII : 306-308.

Freccero, A., 2000 : Roman Painting. Wall Paintings, Fayum Portraits, and Polychrome Statuary. Conservation, Materials and Context, Göteborg.

- 2005 : Pompeian Plasters. Insula I 9 and Forum, Rome.

- 2012a : Pompeian Plasters. Buildings in Regiones I, V, VII, VIII, and IX, Rome.

- 2012 b : "Fragments of wall paintings in San Lorenzo in Lucina", in San Lorenzo in Lucina. The transformations of a Roman quarter, Stockholm, 363-374.

- 2018 : Wall Painting in Pompeii. Plaster, Stucco, Paint, L'Erma, Rome.

- 2018 (forthcoming) : "The Roman Villa of Agios Donatos; Fragments of Wall Paintings", in Thesprotia Expedition IV, The Finnish Institute at Athens (forthcoming).

Gunter, A.C., 1995 : Marble Sculpture, Labraunda 2 :5, Stockholm.

Hayes, J., 2005 : "Late Hellenistic and Roman pottery in the eastern Mediterranean - an overview of recent developments", in M. Berg Brise et L.E. Vaag (éds.), The Relations in the Eastern Mediteranean from the Late Hellenistic Period to Late Antiquity: The Ceramic Evidence, Halicarnassus Studies III, Odense. 
- 1972 : Late Roman Pottery, Londres.

- 1995 : "An Early Roman Well Group from the Troia Excavations 1992”, Studia Troica 5 : 185-196.

- 2000 : "From Rome to Beirut and Beyond: Asia Minor and Eastern Mediterranean Trade Connections", Rei Cretariae Romanae Acta 36 : 285-297.

- 2009 : "Castelporziano Excavations at the Imperial Vicus 1985-7 and 1996-8. Trenches S and SA: pottery finds and lamps", in Catelporziano IV, Oxford : 1-35.

Hayes, J.W., Gebhard, E. et Hemans, F., 1998 : "University of Chicago Excavtions at Isthmia, 1989 : III", Hesperia 67. $4:$ 405-456.

Hellström, P., 2007 : Labraunda. A guide to the Karian sanctuary of Zeus Labraundos, Istanbul.

- 2009 : "Sacred architecture and Karian identity", in Fr. Rumscheid (éd.), Die Karer und die Anderen, Internationales Kolloquium an der Freien Universität Berlin, 13. Bis 15. Oktober 2005, Bonn : 267-290.

- 2011 : "Feasting at Labraunda and the chronology of the Andrônes", in L. Karlsson et S. Carlsson (éds), Labraunda and Karia (Boreas 32), Uppsala : 149-157.

Hellström, P. et Karlsson, L., 2005 : "Labraunda 2003”, KST 26 : 75-80.

Henry, O., 2018 : "Sanctuaire et pouvoir : nouvelles pistes de réflexion à partir des recherches archéologiques récentes sur le site de Labraunda en Carie (Turquie)", CRAI 2017, à paraître.

Henry, O. et al., 2013 : "Labraunda 2012 - Rapport Préliminaire", Anatolia Antiqua XXI : 285-355.

- 2015 : "Labraunda 2014", Anatolia Antiqua XXIII : 301-394.

- 2016 : "Labraunda 2015”, Anatolia Antiqua XXIV : $339-457$.

- 2017 : “Labraunda 2016”, Anatolia Antiqua XXV : $187-266$

Henry, O. et Ingvarsson-Sundström, A., 2010 : "The story of a tomb at Labraunda", in L. Karlsson et S. Carlsson (éds.), Labraunda and Karia. Proceedings of the International Symposium. Commemorating Sixty Years of Swedish Archaeological work in Labraunda, Boreas 32, Uppsala : 177-198.

Istenič, J. et Schneider, G., 2000 : “Aegean Cooking Ware in the Eastern Adriatic", ReiCretActa 36 : 341-348.

Jameson, M.H., 1992 : "Agricultural labour in ancient Greece", in B. Wells (éd.), Agriculture in ancient Greece. Proceedings of the seventh international symposium at the Swedish Institute at Athens, 16-17 May, 1990, Stockholm : 135-146.

Jones, J.E. et al., 1973 : “An Attic country house below the cava of Pan at Vari", BSA 68 : 356-452.

Karlsson, L., 2011 : "The forts and fortifications of Labraunda", in L. Karlsson et S. Carlsson (éds), Labraunda and Karia (Boreas 32), Uppsala : 217-252.

Landstätter, S., 2003 : "K. Keramik", in C. Lang-Auinger (éd.), Hanghaus in Ephesos I, Vienne : 22-85.

- 2005 : “XIV. Keramik”, in H. Thür (éd.), Hanghaus 2 in Ephesos. Die Wohneinheit 4. Baubefund, Ausstattung, Funde, Vienne, FiE VIII/6 : 230-358.
- 2010 : “A.X. Keramik”, in F. Krinzinger (éd.), Die Wohneinheiten 1 und 2. Baubefund, Ausstattung, Funde, Vienne, FiE VIII/8 : 172-279.

Liljenstolpe, P. et Schmalensee, P., 1996 : “The Roman stoa of Poleites at Labraynda. A report on its architecture", Opuscula Atheniensia 21 : 125-148.

Lungu, V., 2016 : "Céramique", in O. Henry et al., "Labraunda 2015 ", Anatolia Antiqua XXIV : 363-383.

- 2017 : "Céramique", in O. Henry et al., "Labraunda 2016 ", Anatolia Antiqua XXV : 233-245.

Mlynarczyk, J., 2014 : "LRC (Phocean) ware pottery in the urban context of Hippos - Susita", in H. Meyza et K. Domzalski (éds.), Late Hellenistic to Medieval Fine Ware of the Aegean Coast of Anatolia, Varsovie : 193-200.

Morris, S.P. et Papadopoulos, J.K., 2005 : "Greek towers and slaves: An archaeology of exploitation", $A J A$ 109:2 : 155-225.

Olcese, G. et al. 2002 : Le ceramiche comuni a Roma $e$ in area romana (III sec. A.C.-I/II sec. D.C.). Produzione, circolazione, tecnologia (= Documenti di archeologia 17), Mantova.

Opaiţ, A., 2004 : Local and Imported Ceramics in the Roman Province of Scythia (4th-6th centuries AD), BAR IS 1274, Oxford.

- 2014 : "Defining more Toman Amphora Types from the Athenian Agora: Too much History, too little Typology (I)", Rei Cretariae Romanae Fautorum Acta 43: 43-54.

Peignard-Giros, A., 2012 : "Les céramiques communes à Délos à l'époque hellénistique tardive $\left(\mathrm{II}^{\mathrm{e}}-\mathrm{I}^{\mathrm{er}}\right.$ siècles av. J.-C.)", in Les céramiques communes dans leur contexte régional : faciès de consommation et mode d'approvisionnement. Actes de la table ronde organisée à Lyon les 2 et 3 février 2009 à la Maison de l'Orient et de la Méditerranée. Lyon : Maison de l'Orient et de la Méditerranée Jean Pouilloux : 243-256.

Peschlow-Bindokat, A., 1996 : Der Latmos. Eine unbekannte Gebirgslandschaft an der türkischen Westküste, Mainz.

- 2014 : Herakleia on the Latmos City and Environment, Istanbul.

Peschlow-Bindokat, A. et Gerber, C., 2012 : “A Carian Mountain Landscape: The Latmos-Beşparmak Mountains Sites with Early Rock Paintings", in M. Özdoğan, N. Başgelen et P. Kuniholm (éds.), Western Anatolia, The Neolithic in Turkey, V4, Istanbul : 67-115.

Pohl, I., 1987 : Materiali rinvenuti in Piazzale delle Corporazioni, portico ovest: saggi sotto o mosaici, «S »1978, Supplemento, 1987 : 165-443, 216-443.

Proust, C., 2009 : "Méthodologie d'étude des restes organiques minéralisés", in B. Bizot et M. Signoli (dir.), Rencontre autour des sépultures habillées, Editions des Hautes-Alpes, 2009 : 103-109.

Reynolds, P., 2013 : “Transport amphorae of the First to Seventh Centuries: Early Roman to Byzantine Periods", in W. Aylward (éd.), Excavations at Zeugma, Conducted by Oxford Archaeology, The Packard Humanities Institute, Los Altos, California, Volume II : 93-161, plates $43-74$. 
Rotroff, S.I. et Oliver, A.Jr, 2003 : The Hellenistic Pottery from Sardis: the Finds through 1994, Cambridge (Mass.), Londres.

Sassoni, E. et al., 2017 : "Some recent findings on marble conservation by aqueous solutions of diammonium hydrogen phosphate", Materials Issues in Art and Archaeology XI 2 (37-38) : 2021-2026.

Siebert, A.V., 1999 : Instrumenta Sacra: Untersuchungen zu römischen Opfer-, Kult- und Priestergeräten, Berlin, New York.

Turnovsky, P., 2005 : “The morphological repertory of Late Roman/Early Byzantine coarse wares in Ephesos", in J.M. Gurt i Esparraguera, J. Buxedai Garrigos et M.A. Cau Ontiveros (éds.), $L R C W$ 1. Late Roman coarse wares, cooking wares and amphorae in the Mediterranean: Archaeology and archaeometry (BAR-IS, 1340), Oxford : 635-654.

Vitruvius : Zehn bücher über Architectur. Translated to German by C. Fensterbusch, Darmstadt 1964.
Westholm, A., 1963 : The architecture of the Hieron (ActaAth-4 ${ }^{\circ}, 5: 1: 2$ ), Labraunda I:2, Lund.

Wicenciak, U., 2014 : "Pottery Production in the Late Hellenistic and Early Roman Periods at Jiyeh, Roman - ancient Porphyreon (Lebanon)", in B. FischerGenz, Y. Gerber et H. Hamel, (éds.), Pottery in the East. Local Production and Regional Trade. Proceedings of the round table held in Berlin, 19-20 February 2010, Archeopress : 103-124.

- 2016 : Porphyreon. Hellenistic and Roman Pottery production in Sidon hinterland, Varsovie.

Winther-Jacobsen, K., 2010 : "The classical farmstead revisited. Activity differentiation based on a ceramic use-typology", BSA $105:$ :269-290.

Zabehlicky-Scheffenegger, S. et Schneider, G., 2005 : "Ephesian Cooking Vessels of the Augustan Period", in M. Berg Briese et L.E. Vaag (éds.), Trade Relations in the Eastern Mediterranean from the Late Hellenistic Period to Late Antiquity: The Ceramic Evidence, Halicarnassian Studies III, Odense : 63-67. 


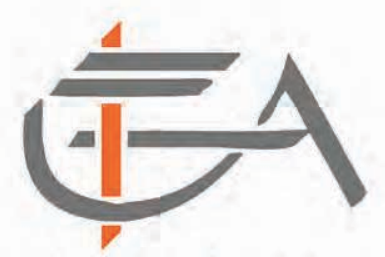

Institut Français d'Etudes Anatoliennes

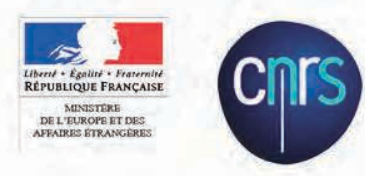

La citadelle de Tushpa (Van, Turquie).

ISBN: 978-2-36245-074-7

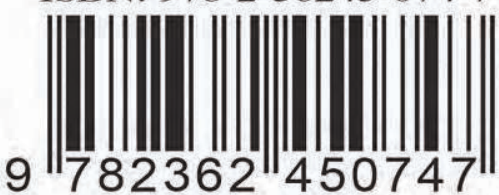

